\title{
Glial Cell Plasticity During Development and Myelinopathies
}

\author{
Angela Dawn Morris \\ Grottoes, Virginia
}

M.S., Old Dominion University, 2007

B.S., Old Dominion University, 2006

A Dissertation presented to the Graduate Faculty

of the University of Virginia in Candidacy for the Degree of

Doctor of Philosophy

Department of Biology

Neuroscience Graduate Program

University of Virginia

May, 2017 


\begin{abstract}
Forming a functional vertebrate nervous system requires intricate interactions amongst various cell populations, including neurons and glia. Diverse populations of glial cells, such as myelinating glia, i.e., oligodendrocytes of the central nervous system (CNS) and Schwann cells and motor exit point (MEP) glia of the peripheral nervous system (PNS), and perineurial glia, which form the blood-nerve barrier, comprise the nervous system. Myelin sheaths promote rapid and efficient communication between the CNS and PNS, and myelinopathies have a significant impact on nervous system function. Because of the functional deficits that can result following myelinopathies, it is imperative to develop a better understanding of the glial interactions and responses following myelin perturbations.
\end{abstract}

My work presented here provides a detailed characterization of glial-glial interactions during spinal motor nerve assembly, maintenance and in response to axonal injury, as well as glial responses following a demyelinating insult. First, I describe modifications made to a transmission electron microscopy (TEM) protocol that allows me to efficiently locate nervous system structures to investigate glial ultrastructure and morphology. Next, I use this TEM assay, in combination with in vivo imaging, immunohistochemistry and laser axonal transection and PNS barrier integrity assays to better characterize glial-glial interactions in response to a myelinopathy in which oligodendrocyte progenitor cells (OPC) ectopically exit the CNS to associate with peripheral axons. To investigate glial response following a demyelinating insult, I present data characterizing a novel focal 
demyelination model in zebrafish that I created to visualize myelin breakdown and repair in vivo. Taken together, this work provides a more comprehensive understanding about the remarkable plasticity of glial cells during nervous system development, maintenance and in response to myelinopathies. 


\section{Dedication}

Words cannot describe how grateful and appreciative I am to all of my family, loved ones, mentors and friends for their continuous support and encouragement as I pursued this dream. Praise God for blessing me with this opportunity and for giving me the dedication, strength and commitment to achieve this goal. I also want to thank the best puppy dogs in the world, Hunter and Diesel, for filling my life with joy and unconditional love. Life is definitely better with a dog, and I am so fortunate for the amazing friends, family, loved ones and life that I have! Cheers to new adventures with all of my favs...you know who you are! 


\section{Table of Contents}

\section{Introduction: Glial responses during development and myelinopathies ...............1}

Development and organization of spinal motor nerves ....................................2

Glial cells in nervous system development and maintenance...............................

i. Myelinating glia of the CNS and PNS .................................................. 8

ii. Myelin sheath: a specialized structure ….................................................

iii. Axonal and myelinating glial interactions............................................ 10

Perineurial glia: imperative for PNS development \& function.............................15

i. Debates surrounding perineurial origin ................................................ 15

ii. Nervous system barriers .................................................................... 18

Peripheral glial-glial interactions in motor nerve assembly, maintenance and injury

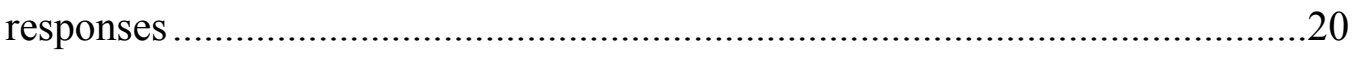

i. Glial-glial interactions in development and nervous system maintenance .....20

ii. Glial-glial interactions in motor nerve injury and regeneration...................23

CNS Demyelination: Oligodendrocytes under attack ....................................25

i. Myelin breakdown in the CNS .............................................................25

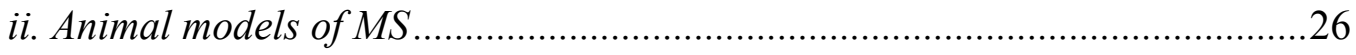

Zebrafish: a powerful model system with unique advantages ..............................30

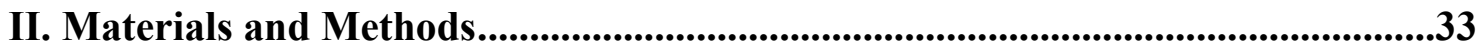

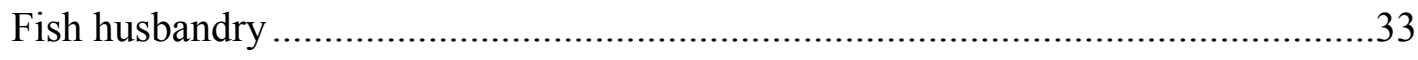

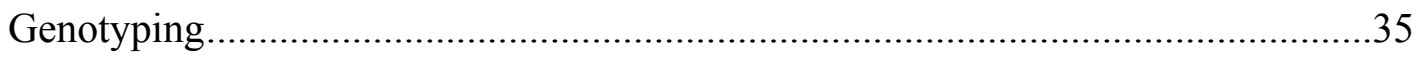

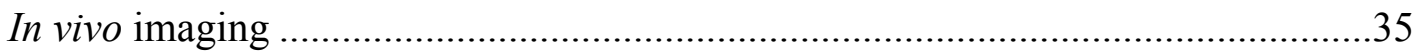




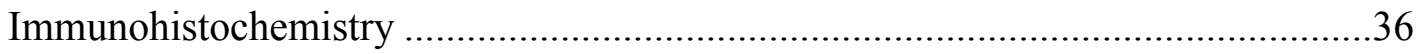

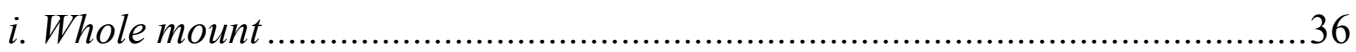

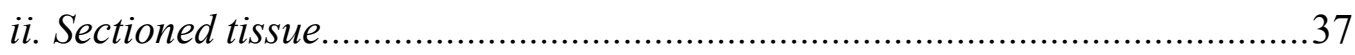

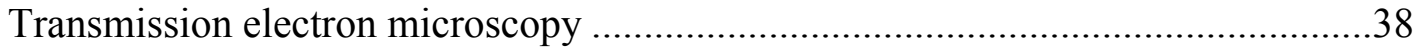

i. Transmission electron microscopy equipment and materials..........................40

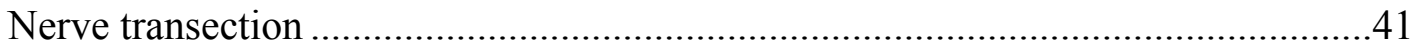

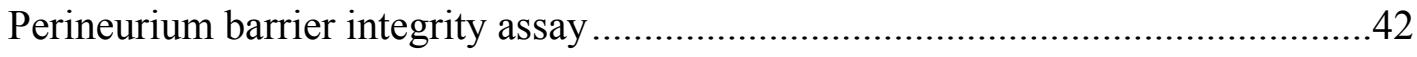

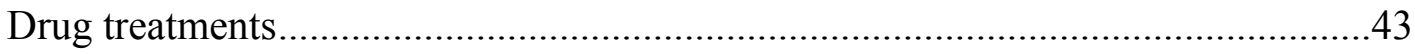

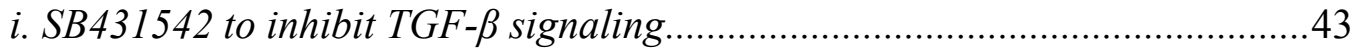

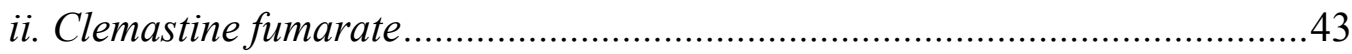

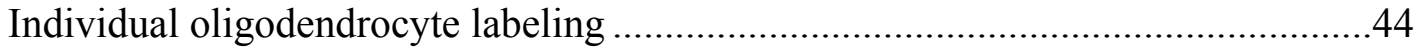

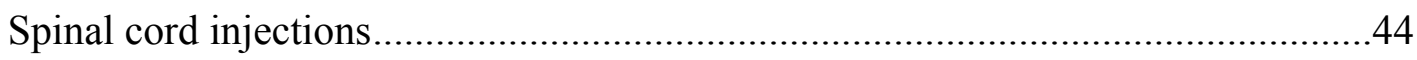

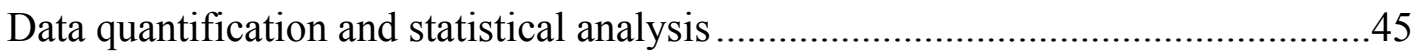

III. Transmission electron microscopy of zebrafish spinal motor nerve roots .......46

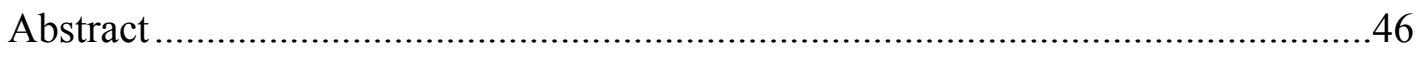

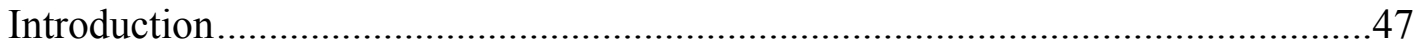

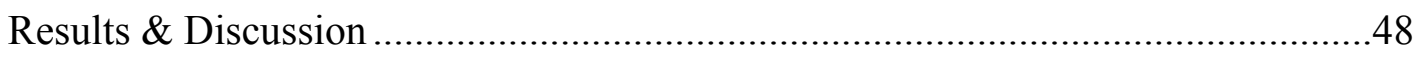

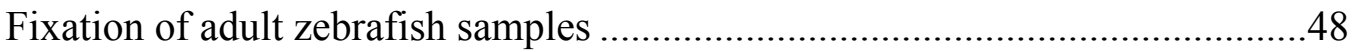

Sample preparation for microtome sectioning ..................................................50

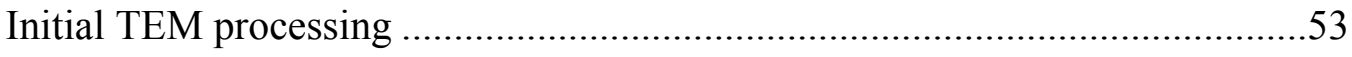

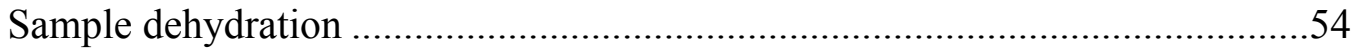

TEM processing for ultrathin coronal/frontal cross-sections...............................54

TEM processing for ultrathin transverse sections...............................................56 
TEM imaging of spinal motor nerve roots................................................58

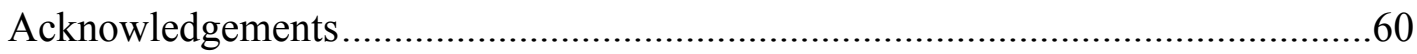

\section{Perineurial glial plasticity and the role of TGF- $\beta$ in the development of the}

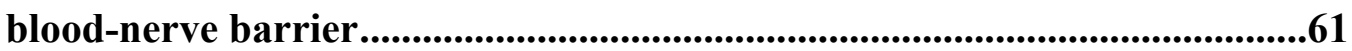

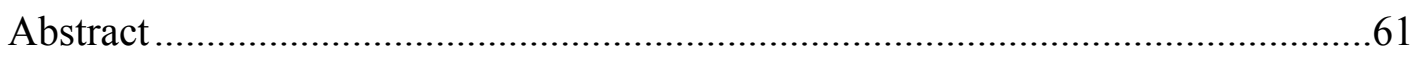

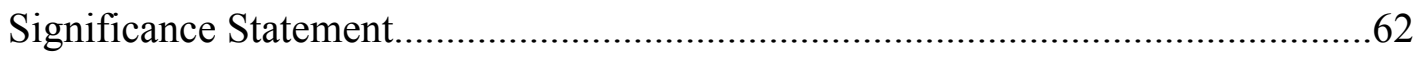

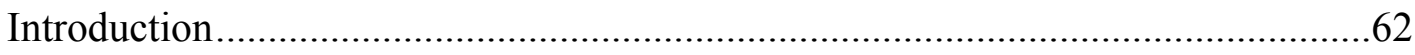

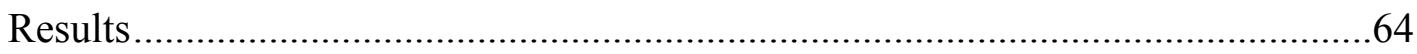

$e r b b 3 b^{-/}$spinal motor nerves as a model to study perineurial glial plasticity in

response to myelin perturbations …...............................................6

Perineurial glia exit the spinal cord in the presence of ectopic

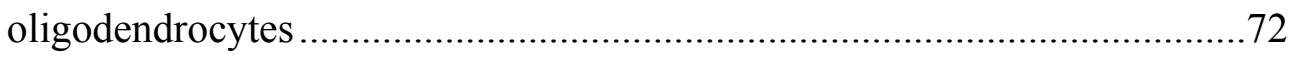

Ectopically located OPCs affect perineurial glial response to injury ...............77

Perineurial glia have an increase in ZO-1 labeling along $e r b b 3 b^{-/-}$spinal motor

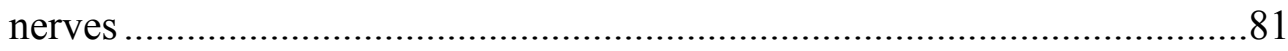

TGF- $\beta 1$ partially mediates the increase in ZO-1 labeling along erbb3b $b^{-/-}$motor

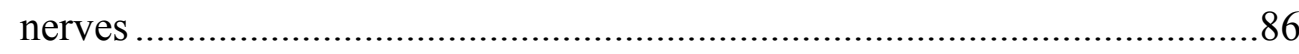

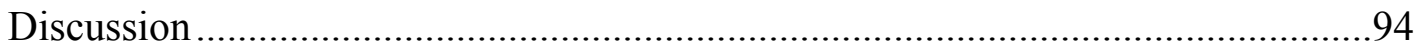

Is erbb3b an important mechanism for mediating perineurial glial development

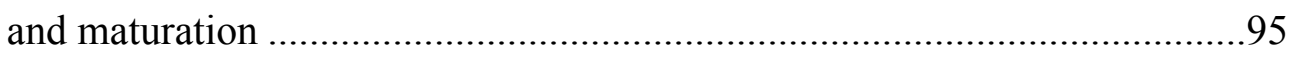

Do ectopically-located OPCs impair perineurial glial injury responses? ..........97

Do ectopic OPCs increase zonula occludens-1 labeling within the bloodnerve barrier. 


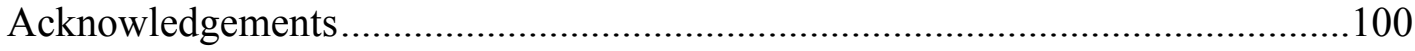

V. Visualizing demyelination in vivo in a novel drug-induced demyelination model in zebrafish ..................................................................................................101

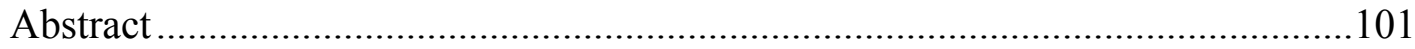

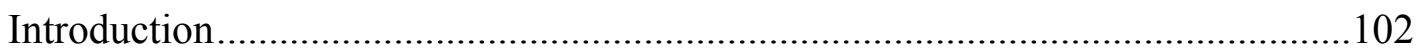

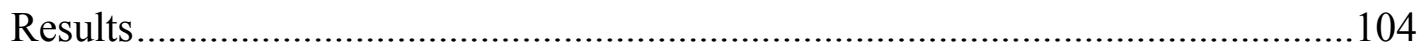

Creating a focal demyelination model in zebrafish .........................................104

Lysolecithin alters the number of Sox $10^{+}$cells within the lesion area ..............105

Lysolecithin induces myelin membrane changes in zebrafish larvae...............110

Axons are spared following lysolecithin injections .........................................113

Professional phagocytes are recruited to the injury site....................................113

Oligodendrocyte cytoskeleton dynamics following exposure to a demyelinating

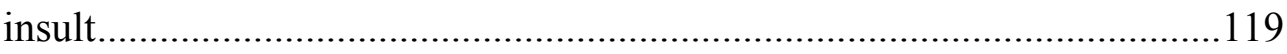

Clemastine fumarate minimizes the effects of myelin membrance changes following exposure to lysolecithin............................................................120

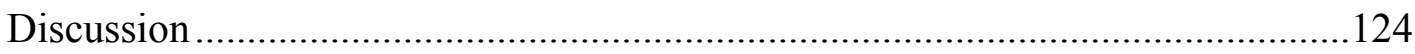

Animal models for recapitulating the heterogeneity of MS...............................124

Observing myelin membrane changes in vivo .................................................126

Professional phagocyte response following the demyelinating insult ..............129

Clemastine fumarate promotes myelin repair ....................................................131

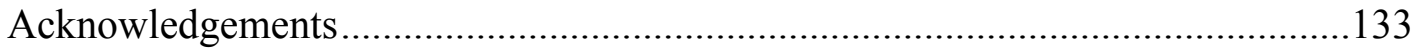

VI. Discussion and future directions ..................................................................................134

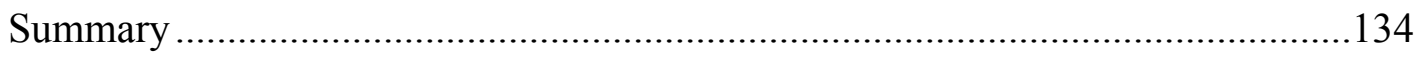




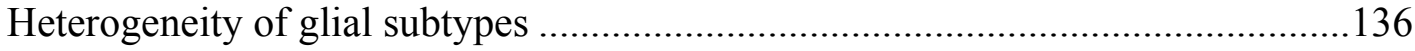

Do ectopic oligodendrocytes maintain their CNS profile and characteristics? ......139

The perineurium: an understudied structure and glial cell..............................145

Identifying mechanisms involved in perineurial developmental, differentiation and

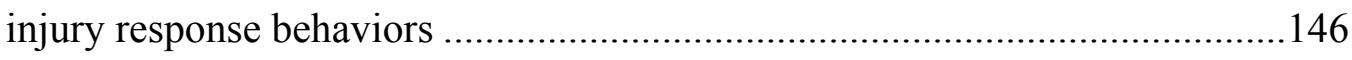

Oligodendrocyte cytoskeletal responses following a demyelinating insult............152

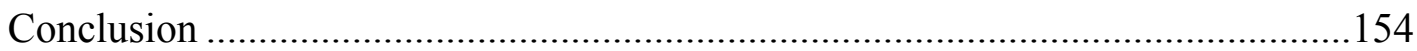

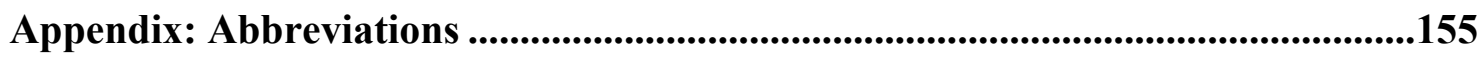

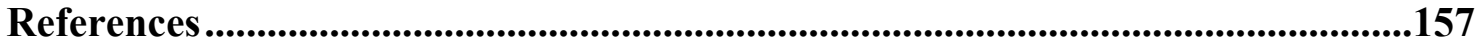




\section{Figures and Tables}

\section{Introduction: Glial responses during development and myelinopathies}

Figure 1-1 Zebrafish: a powerful model for investigating nervous system development, maintenance and injury responses in vivo .................................

Figure 1-2 Origin and cell populations that comprise spinal motor nerves ...............5

Figure 1-3 Nodes of Ranvier promote rapid action potential propagation ...............11

Figure 1-4 Perineurium ensheaths axons and their myelin sheaths ........................16

\section{Materials and Methods}

Table 2-1 Descriptions and abbreviations of transgenic and mutant lines ..............34

\section{Transmission electron microscopy of zebrafish spinal motor nerve roots}

Figure 3-1 Spinal motor nerves in adult zebrafish

Figure 3-2 Vibrating blade microtome transverse sections of adult zebrafish trunk samples

Figure 3-3 Locating spinal motor nerves in adult zebrafish transverse sections ......55

Figure 3-4 Embedding transverse sections for ultrathin sectioning in coronal/frontal and transverse orientations

Figure 3-5 Ultrastructural evaluation of adult zebrafish motor nerves..... .59

IV. Perineurial glial plasticity and the role of TGF- $\beta$ in the development of the

blood-nerve barrier

Figure 4-1 Perineurial glial development in zebrafish. 65

Figure 4-2 OPCs ectopically exit the spinal cord in $e r b b 3 b^{-/-}$larvae.......................70

Figure 4-3 Perineurial glial proliferation is normal in erbb3 $b^{-/}$larvae .74 
Figure 4-4 Perineurial glial morphology is altered in $e r b b 3 b^{-/}$zebrafish .76

Figure 4-5 Perineurial glial recruitment to the injury site is impaired on axonal tracts with centrally-derived OPCs

Figure 4-6 Perineurial glia form phagocytic vesicles and glial bridges in the absence of all myelinating glial cells

Figure 4-7 Perineurial glia have more tight junctional proteins in erbb3b mutant zebrafish

Figure 4-8 Perineurial barrier integrity is compromised in adult erbb3 $b^{-/-}$ zebrafish

Figure 4-9 Phosphorylated Smad3 levels are increased along erbb3b $b^{-/}$larval spinal motor nerves .90

Figure 4-10 Inhibiting TGF- $\beta 1$ decreases ZO-1 labeling along spinal motor nerves

\section{Visualizing demyelination in vivo in a novel drug-induced demyelination model} in zebrafish

Figure 5-1 Focal delivery of solutions into the zebrafish spinal cord 106

Figure 5-2 Lysolecithin effects on Sox $10^{+}$cells 108

Figure 5-3 Myelin membrane changes within lysolecithin lesion. 111

Figure 5-4 Axons are spared within the lysolecithin dispersal region 114

Figure 5-5 Professional phagocytes traverse through myelin layers 116

Figure 5-6 Professional phagocytes proliferate and clear Sox $10^{+}$debris 118

Figure 5-7 Oligodendrocyte cytoskeletal components dynamically change following exposure to lysolecithin..... 
Figure 5-8 Clemastine reduces myelin membrane changes following a

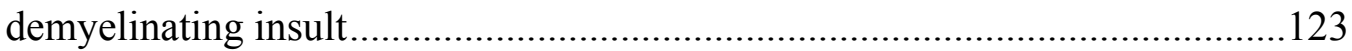

\section{Discussion and future directions}

Figure 6-1 Myelin Basic Protein (MBP) labeling along wildtype and $e r b b 3 b^{-/-}$

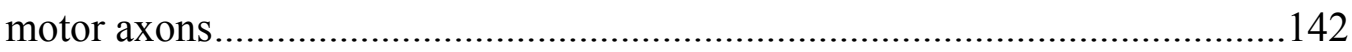

Figure 6-2 Glial cells associated with $n r g^{z 26-/}$ spinal motor axons.........................144 


\section{Chapter 1}

\section{Introduction: Glial responses during development and myelinopathies}

Forming and maintaining a functional vertebrate nervous system is a complex process requiring orchestrated interactions by several cell populations, including glial cells. The vertebrate nervous system is divided into the central nervous system (CNS), which includes the brain and spinal cord, and the peripheral nervous system (PNS), consisting of the autonomic, enteric, motor and sensory systems (Dyck \& Thomas, 2005). The PNS relays sensory information from the periphery to the CNS (afferent), and distributes motor information from the CNS (efferent) to target organs, glands or muscles via sensory and motor nerves, respectively.

Efficient communication between the CNS and PNS is imperative for a functional nervous system, and spinal motor nerves are important conduits allowing for rapid propagation of action potentials from the CNS to innervate peripheral targets, including muscle. Disorders affecting glial cells, including myelinopathies or demyelinating insults, have a significant impact on nervous system function and impair rapid action potential, or signal propagation. Because of these functional consequences, developing a better understanding about the interactions and responses of glial cells following myelin perturbations, both during development and in maintenance, is imperative. Using a vertebrate model system like zebrafish (Figure 1-1) allows for visualizing cell interactions, behaviors and responses in vivo, identifying processes or mechanics that are 
essential for forming and maintaining a functional nervous system in real-time. Thus, in this dissertation, I use zebrafish as a model organism for investigating:

1) Glial-glial interactions during PNS spinal motor nerve assembly, response to injury and in nerve maintenance

2) Glial response following a demyelinating insult in the CNS

In this introduction, I will review the main glial cell populations relevant for my studies, i.e., CNS and PNS myelinating glial cells and perineurial glia of the PNS, and will discuss their roles in nervous system development, maintenance and response to a peripheral axonal transection injury or CNS demyelinating insult.

\section{Development and organization of spinal motor nerves}

During development, precisely coordinated and orchestrated interactions by various cell populations, including motor axons (Figure 1-1B) and their ensheathing glia, endoneurium, perineurium and epineurium, are needed to form a functional motor nerve (Akert et al., 1976; Appel et al., 1995; Bourne, 1968; Corfas et al., 2004; Gamble \& Breathnach, 1965; Kucenas et al., 2008b; Shanthaveerappa \& Bourne, 1966). CNSderived motor exit point (MEP) glia and neural crest-derived Schwann cells are responsible for myelinating spinal motor axons (Emery, 2010; Jessen \& Mirsky, 2005; Smith et al., 2014). The endoneurium surrounds myelinated axons and the perineurium bundles myelinated and non-myelinated axons to form a nerve fascicle. The outermost 
A

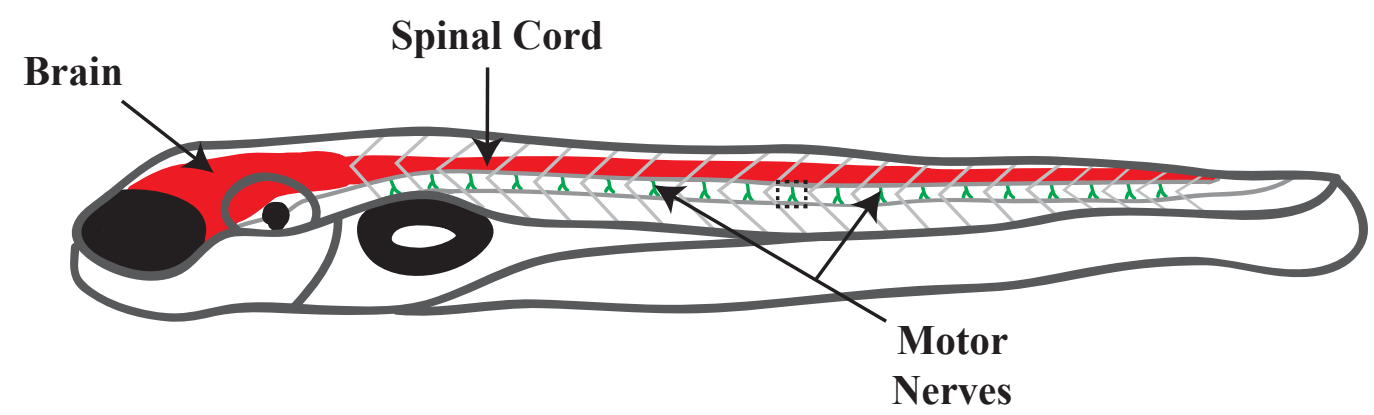

B

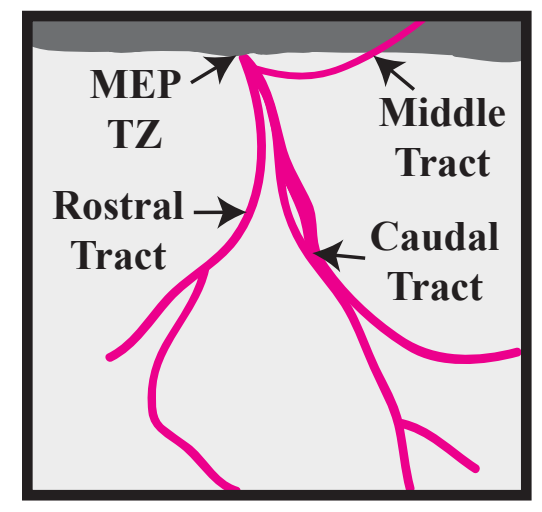

Figure 1-1. Zebrafish: a powerful model for investigating nervous system development, maintenance and injury responses in vivo. (A) Cartoon of a zebrafish larvae identifying the CNS, consisting of the brain and spinal cord (red), and the spinal motor nerves (green). Dotted box denotes the region represented in (B). (B) Schematic of the zebrafish spinal motor nerve. Motor axons (magenta) exit the spinal cord via the motor exit point transition zone (MEP TZ). The rostral and caudal tracts extend ventrally and the middle tract extends dorsally. 
layer, the epineurium, encases several nerve fascicles to form the entire spinal motor nerve (Figure 1-2A).

The different cell populations that comprise motor nerves also have distinct origins (Figure 1-2B). Motor neurons are specified from precursors in the pMN domain of the spinal cord and their axons exit the CNS via MEP transition zones to project to peripheral targets (Rowitch, 2004). Once in the periphery, local cues help the axons path-find until their target cells are reached and innervated. In zebrafish, primary motor neurons are specified first and, upon innervation of their targets, survival behaviors like swimming can be performed. There are three primary motor neurons that project axons through the motor exit point (MEP) transition zone at each iterated segment along the zebrafish trunk. The rostral and caudal tracts project axons ventrally and the middle tract projects dorsally (Eisen, 1991; Myers et al., 1986) (Figure 1-1B). Shortly after primary motor neuron specification and axonal pathfinding has occurred, secondary motor neurons are specified and extend axons through the MEP transition zones to innervate targets. Upon completion of axonal development, each MEP transition zone will have approximately 70 motor axons (Eisen, 1991; Lin et al., 2001; Myers et al., 1986), and large caliber axons will be myelinated by peripheral myelinating glia, called MEP glia and Schwann cells.

The peripheral myelinating glial cells also have distinct and unique origins (Figure 1-2C). MEP glia, also derived from $\mathrm{pMN}$ precursors in the spinal cord, exit into the periphery via the same MEP transition zones that axons project through and associate with and myelinate the motor root (Smith et al., 2014). Neural crest-derived Schwann cells also 
A
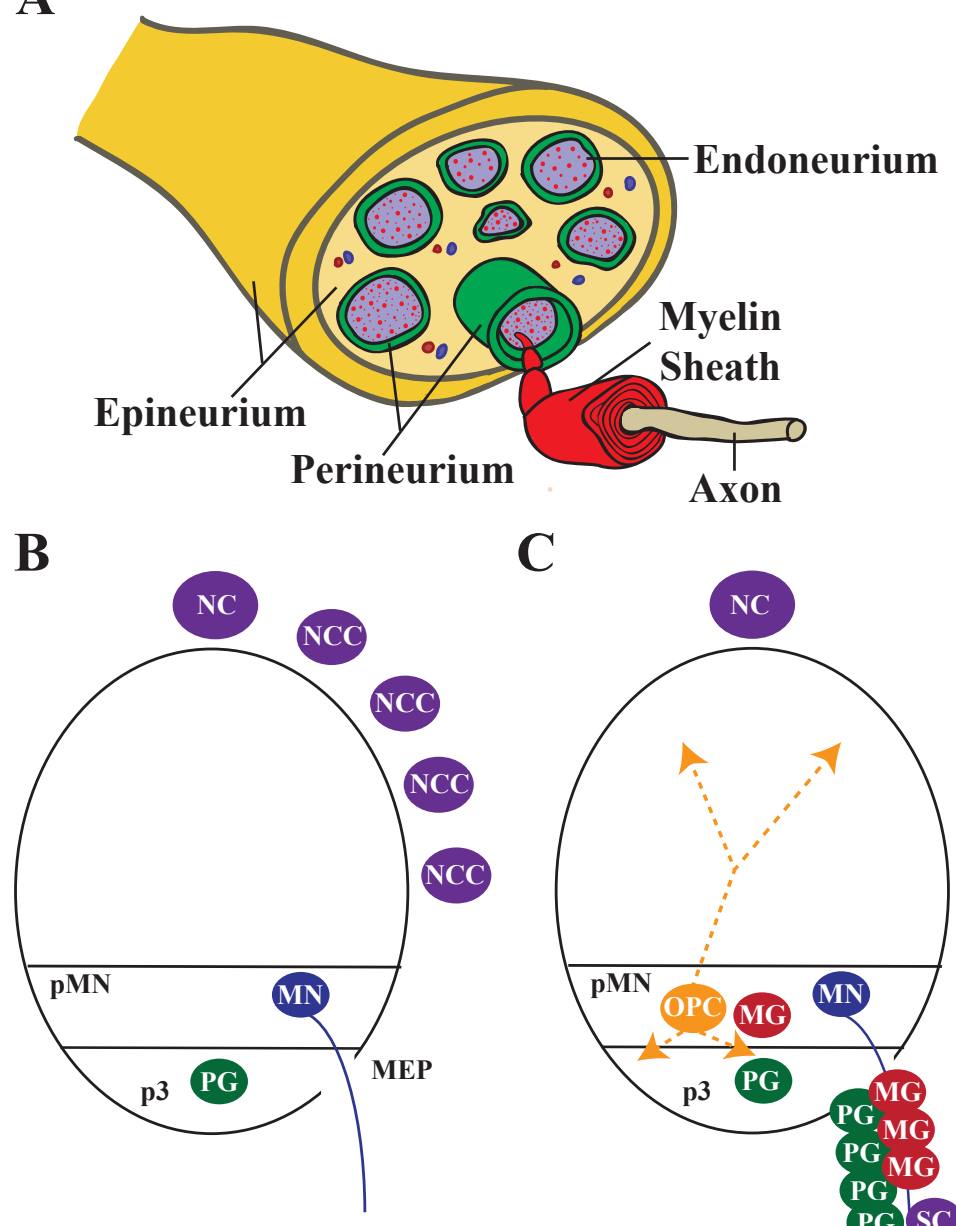

C

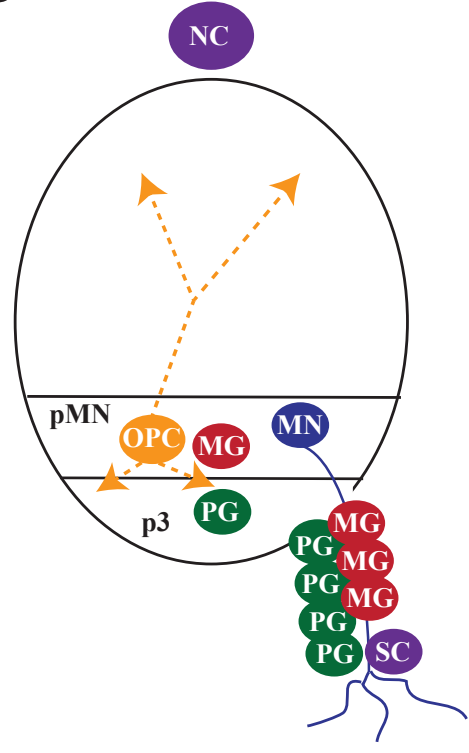

D

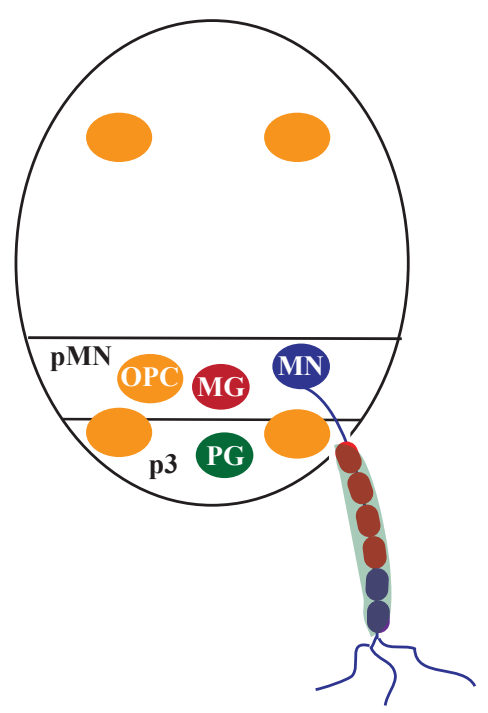

Figure 1-2. Origin and cell populations that comprise spinal motor nerves. (A) Schematic of a motor nerve. The myelin sheath, formed by MEP glia and Schwann cells, ensheath motor axons. Endoneurium surrounds the myelinated axons. Perineurium bundles myelinated axons to form a nerve fascicle. Several nerve fascicles are encased by the epineurium to comprise the motor nerve. (B) Motor neurons (MN) are specified in the pMN domain. The motor exit point (MEP) is the site at which the motor neuron extends its axon into the periphery. Perineurial glia (PG) are derived from precursors in the $\mathrm{p} 3$ domain of the ventral spinal cord. The neural crest (NC) forms at the dorsal neural tube and its cells (NCC) migrate. Some NCC associate with motor axons and eventually differentiate into Schwann cells (SC). MEP glia (MG) and oligodendrocyte progenitor cells (OPCs) are derived from the pMN domain. OPCs migrate dorsally and ventrally (orange arrows) to associate with CNS axons. MG exit into the periphery via the MEP to myelinate axons at the motor root. Perineurial glia extend processes from the spinal cord and eventually migrate from the CNS via the MEP. (D) During differentiation, oligodendrocytes (orange) myelinate dorsal and ventral CNS axons. MG (red) and SC (purple) wrap motor axons and form the myelin sheath. PG ensheath the MG and $\mathrm{SC}$ and eventually mature into the perineurium (green). 
associate with spinal motor axons, as non-myelinating Schwann cells ensheath several small caliber axons to form Remak bundles and myelinating Schwann cells form an insulating, myelin sheath around individual large caliber axons (Jessen \& Mirsky, 2005). Myelin, a specialized structure that promotes quick saltatory conduction of action potentials along axons, is a key element of vertebrate nervous system development that is discussed in greater detail below.

Following MEP glial and Schwann cell association with motor axons, the endoneurium, perineurium and epineurium surround, or encase, these components. Although development of the endoneurium and epineurium are not well understood, fate-mapping studies demonstrate that the endoneurium is derived from neural crest cells (Akert et al., 1976; Joseph et al., 2004) and the epineurium is thought to be mesenchymal in origin (Halata et al., 1990; Haninec, 1988). However, the origin of perineurial glia, the cells that form the perineurium, was of debate until elegant studies in zebrafish and mouse revealed that these cells are derived from the CNS (Clark et al., 2014; Kucenas et al., 2008b). Perineurial glia are derived from the p3 domain of the ventral spinal cord, migrate out of the spinal cord into the periphery via MEP transition zones and bundle many myelinated and unmyelinated axons to form a nerve fascicle (Clark et al., 2014; Kucenas et al., 2008b) (Figure 1-2B - 1-2D). Perineurial glia differentiate to form the mature perineurium, a component of the blood-nerve barrier, and provide protection for the motor axons, acting as both a mechanical and diffusion barrier (Akert et al., 1976; Bourne, 1968; Clark et al., 2014; Jessen \& Mirsky, 2005; Kristensson \& Olsson, 1971; Kucenas et al., 2008b; Rowitch, 2004; Smith et al., 2014). 
Because forming and maintaining a functional motor nerve requires complex precision, it comes as no surprise that defects may arise, resulting in disease states like peripheral neuropathies or myelinopathies. Recently, the role of peripheral glial-glial interactions during spinal motor nerve assembly has emerged as an important component of nervous system development (Binari et al., 2013; Clark et al., 2014; Kucenas et al., 2008b). Specifically, Schwann cells fail to myelinate peripheral axons when perineurial glial cells are perturbed (Binari et al., 2013; Kucenas et al., 2008b). Reciprocally, lack of neural crest-derived Schwann cells results in perineurial defects, and, in some instances, perineurial cells fail to migrate from the CNS (Kucenas et al., 2008b; Kucenas et al., 2009). Based on this line of evidence, the interactions between perineurial glia and peripheral myelinating glial cells are imperative for forming a functional nerve.

Intriguingly, certain peripheral neuropathies result in a phenotype where oligodendrocyte progenitor cells (OPCs), the resident myelinating glial cell of the CNS, ectopically exit the spinal cord, associate with and myelinate peripheral axons (Coulpier et al., 2010; Kucenas et al., 2009; Kucenas et al., 2008b; Smith et al., 2014; Weinberg et al., 1975). Although recruiting endogenous myelinating precursors from their native environments to ectopically myelinate axons battling a myelinopathy has been proposed for quite some time, how the remaining ensheathing cells are impacted by this change remains unknown. Thus, as part of my dissertation, I am specifically interested in whether perineurial glia are capable of compensating for, and maintaining, a functional motor nerve despite the presence of ectopically-located oligodendrocytes. 


\section{Glial cells in nervous system development \& maintenance}

\section{i. Myelinating glia of the CNS and PNS}

Specialized glial cells such as oligodendrocytes and peripheral myelinating glia, including MEP glia and Schwann cells, form the myelin sheath around axons of the CNS and PNS, respectively. These myelinating glial cell populations are derived from separate and unique domains. Oligodendrocytes arise from the dorsal \& ventral neural tube, MEP glia are derived from the pMN domain of the spinal cord and Schwann cells are derived from the neural crest (Figure 1-2B - 1-2C), a transient structure responsible for many peripheral derivatives including PNS neurons, melanocytes, smooth muscle, craniofacial structures and PNS glia (Le Douarin \& Dupin, 2003; Taveggia et al., 2010). Although myelinating glial cells arise from distinct regions and precursors, they go through several similar differentiation stages, starting from a precursor through maturation (Baumann \& Pham-Dinh, 2001; Garbay et al., 2000). During myelination, they extend processes around axons and wrap their plasma membrane to form many tight layers of compact myelin. Within the CNS, one oligodendrocyte can myelinate up to as many as 40-50 individual axonal segments (McTigue \& Tripathi, 2008; Peferoen et al., 2014; Quarles et al., 2006). However, peripheral myelinating glial cells, like Schwann cells, myelinate one axonal internodal segment (Baumann \& Pham-Dinh, 2001; Garbay et al., 2000). Regardless of their origin or residential nervous system, these specialized glial cells are capable of myelinating axons within the CNS (oligodendrocytes) or PNS (MEP glia and Schwann cells). 


\section{ii. Myelin sheath: a specialized structure}

The main function of the myelin sheath is to increase action potential propagation along myelinated axons. Myelin improves the speed and efficiency at which the nervous system can operate, a key component for vertebrate function because smaller-diameter axons achieve rapid impulse propagation simply by being myelinated, thus, eliminating the requirement of larger diameter axons to achieve the same impulse propagation efficiency (Nave, 2010). Myelin is composed of 70-80\% lipids, including cholesterol, galactolipids, phospholipids, choline, sphingomyelin, and fatty acids, as well as $20-30 \%$ proteins (Garbay et al., 2000). This high lipid to protein ratio provides the insulating feature of myelin that is needed for promoting rapid and efficient saltatory action potential propagation.

The lipid classes found within the myelin sheath are similar for CNS and PNS myelin but the amount of each lipid may differ slightly. However, some of the proteins comprising the CNS and PNS myelin differ. For example, proteolipid protein (PLP or DM-20) is present in CNS myelin whereas the protein composition of peripheral myelin mainly consists of the glycoproteins protein 0/P0 and peripheral myelin protein 22/PMP22. However, proteins such as myelin basic protein (MBP) and myelin-associated glycoprotein (MAG) are present within both CNS and PNS myelin (Baumann \& PhamDinh, 2001; Garbay et al., 2000). Regardless of the nervous system, myelinating glial cells are necessary for functional nerve development and maintenance in both the CNS and PNS. 


\section{iii. Axonal and myelinating glial interactions}

Axons and myelinating glial cells interact with one another expressing a variety of signals to regulate the thickness of the myelin sheath as well as the proper formation of nodes of Ranvier that are critical components of action potential propagation (Quarles et al., 2006). Nodes of Ranvier are unmyelinated gaps residing between adjacent myelinating glial cells. This region is enriched with voltage-gated sodium $\left(\mathrm{Na}^{+}\right)$channels that promote saltatory conduction of action potentials. Paranodes flank the nodes of Ranvier and make up the axo-glial junction between the axolemma and the myelinating glial cell. Paranodes also act as a barrier to separate the nodal $\mathrm{Na}^{+}$channel enriched region from the juxtaparanodal potassium $\left(\mathrm{K}^{+}\right)$channels to ensure each remain within their respective locations. Flanking the $\mathrm{K}^{+}$channel enriched juxtaparanodal region and residing within the compact myelin sheath is the internode. The internode is the largest domain and ensures current flow remains within the nodal regions. Thus, impulses jump from one nodal region to the next for rapid and saltatory action potential propagation (Figure 1-3) (Buttermore et al., 2013; Salzer et al., 2008). The interaction between the myelin sheath and the axon is crucial for proper voltage gated channel formation and localization.

Myelinating glial cells also provide the axon with trophic signals, including glial cellderived growth factor (GDNF) (Baloh et al., 1998; Wilkins et al., 2003). These trophic signals have been shown to promote myelination. Additionally, the glycoprotein MAG found in both CNS \& PNS myelin also provides support to the axon because axonal degeneration occurs in its absence (Taveggia et al., 2010). Reciprocally, the axon 


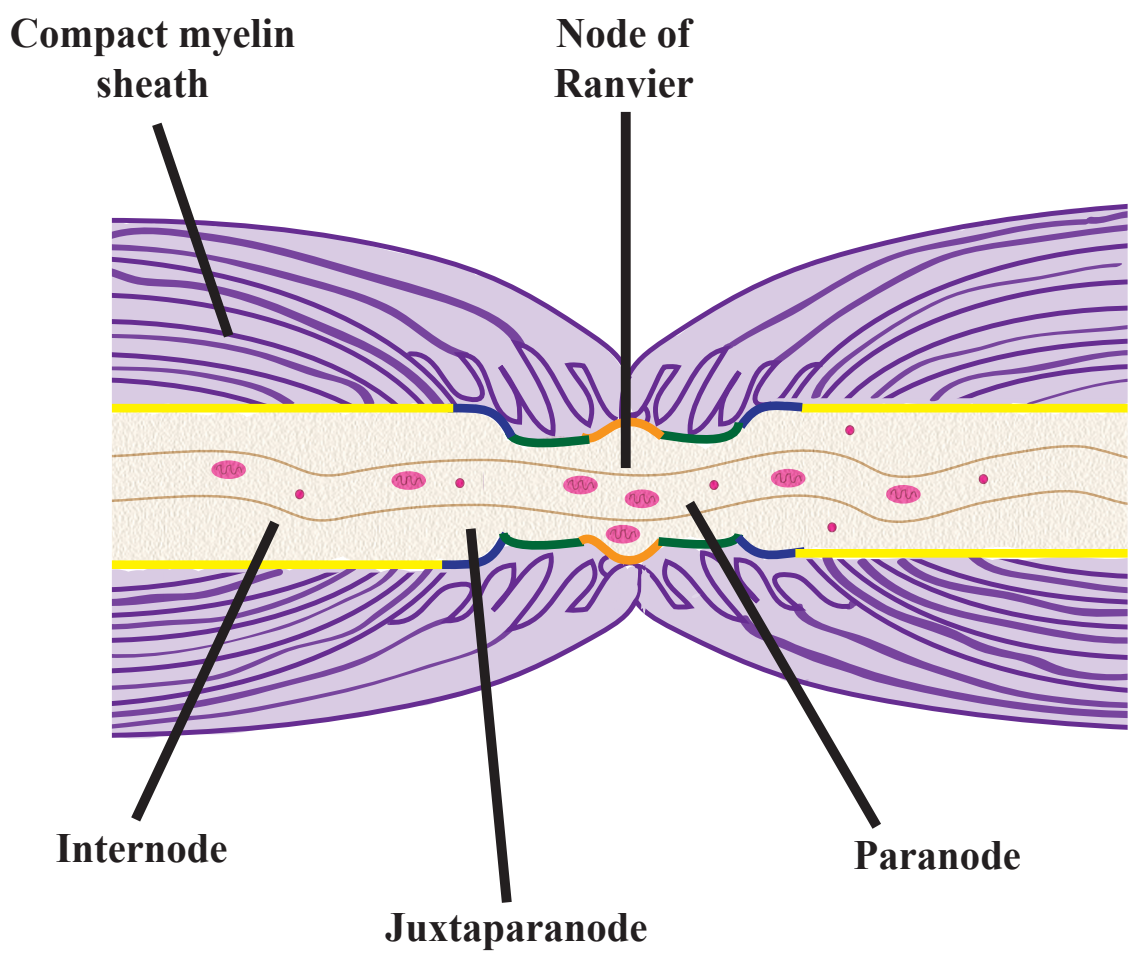

Figure 1-3. Nodes of Ranvier promote rapid action potential propagation. The axon is divided into molecular domains containing channels or molecules important for neuronal function. The internode is located under the compact myelin sheath, formed by oligodendrocytes in the CNS and MEP glia and Schwann cells in the PNS. The juxtaparanodal region is enriched in voltage gated potassium channels as well as contactin associated protein 2 . The paranode acts as a barrier to separate the juxtaparanodal \& nodal domains and contains axonal contact associated protein. The node of Ranvier is the region where the cytoplasmic arms of the myelinating glial cells meet. The node is unmyelinated and exposes the axon to its external environment. Voltage gated sodium channels are abundant within this region. The axonal cytoskeleton contains ankyrin G, providing the node with structural stability. Internode: yellow line, Juxtaparanode: green line, Paranode: blue line, node of Ranvier: orange line.

Adapted from Salzer et al., 2008. 
expresses factors important for myelinating glial cell development. Some of the signals expressed by axons provide similar responses in both the CNS and PNS; however, there are also instances in which the same signal results in different responses depending on the nervous system the axon resides within. For example, PNS axons express Neuregulin 1 Type III, an epidermal growth factor critical for Schwann cell migration, proliferation and differentiation. Within the CNS, the role of Nrg1 Type III in myelination is not well understood and it appears this axonally-expressed ligand is not as critical for initiating oligodendrocyte myelination (Taveggia et al., 2010) whereas other processes, including OPC spacing (Hughes et al., 2013; Kirby et al., 2006; Rosenberg et al., 2008), electrical activity (Emery, 2010; Gibson et al., 2014; Hines et al., 2015; Mensch et al., 2015; Wake et al., 2011), and professional phagocyte cues promote CNS myelination (Miron et al., 2013; Ruckh et al., 2012). However, in the PNS, depleting the axonally-expressed Nrg1 Type III or its Schwann cell heterodimer receptor complex ErbB2/ErbB3 results in detrimental effects on PNS myelination.

Neuregulin-1 is a member of the epidermal growth factor-like family of ligands and has more than 15 isoforms that are membrane associated or secreted (Nave \& Salzer, 2006). Nrg1 Type III is a transmembrane associated isoform expressed abundantly on motor and sensory nerves (Nave \& Salzer, 2006). Axonally-expressed Nrg1 Type III contains an EGF domain that binds to the heterodimer receptor complex formed between the ErbB2 and ErbB3 tyrosine kinase receptors (RTKs). As mentioned above, the complex formed between Nrg1 Type III and ErbB2/ErbB3 is important for Schwann cell migration, proliferation and myelination (Michailov, 2004; Nave \& Salzer, 2006; Perlin et al., 
2011). Furthermore, the amount of Nrg-1 Type III expression has been shown to correlate with myelin sheath thickness, thus, overexpression results in hypermyelination and depletion causes hypomyelination (Michailov, 2004; Taveggia et al., 2005).

Similarly, mutations affecting ErbB2 or ErbB3 have similar developmental phenotypes as Nrg1 Type III mutations, i.e., ErbB2 or ErbB3 mutations also result in Schwann cell migration, proliferation and myelination defects (Birchmeier, 2009; Chen et al., 2006; Garratt et al., 2000b; Lyons et al., 2005; Morris et al., 1999; Nave \& Salzer, 2006; Newbern \& Birchmeier, 2010; Woldeyesus et al., 1999). In ErbB3 mutant mice, peripheral nerves lack Schwann cells eventually resulting in sensory and motor neuron degeneration (Birchmeier, 2009; Riethmacher et al., 1997a). Interestingly, motor and sensory neurons develop normally within the mutants, but the lack of Schwann cell ensheathment has been identified as an important component leading to the axonal degeneration (Birchmeier, 2009). Similarly, depletion of ErbB2 results in Schwann cells that fail to differentiate causing axonal hypomyelination that eventually leads to axonal degeneration (Garratt et al., 2000b; Morris et al., 1999; Woldeyesus et al., 1999). Since ErbB2 is imperative for normal cardiac development, ErbB2-/- mice die before E11 due to cardiac trabeculation defects (Lee et al., 1995). To investigate the effects of ErbB2-/on nervous system development, conditional mutants were created to rescue the cardiac defects (Morris et al., 1999; Woldeyesus et al., 1999). In the rescued mutants, motor neurons formed normally but degenerated at later stages in development (Morris et al., 1999; Woldeyesus et al., 1999). Furthermore, motor axons project to peripheral targets in Erbb2 mutant mice but the projections are disorganized and defasciculated, suggesting 
neuronal function may also be impaired (Woldeyesus et al., 1999). The lack of Schwann cells associated with peripheral nerves may also be responsible for the axonal defasciculation defects observed in the $\operatorname{ErbB} 2$ mutant mice.

The myelination defects in ErbB2 and ErbB3 mouse mutants have also been observed in zebrafish carrying similar mutations. Both $e r b b 2^{s t 61}$ and $e r b b 3 b^{s t 48}$ mutations cause hypomyelination, Schwann cell migration defects and reduction of myelin basic protein $(\mathrm{mbp})$ along the posterior lateral line $(\mathrm{pLLn})$, a mechanosensory organ in zebrafish (Lyons et al., 2005). This work further supports the importance of a functional erbb/nrg1 type III complex throughout Schwann cell development and maintenance (Lyons et al., 2005). However, a limitation to the study by Lyons et al. (2005) is that all observations involved Schwann cells associated with the posterior lateral line (pLLn), the mechanosensory system within zebrafish. Interestingly, erbb3b $b^{-/}$zebrafish are homozygous viable suggesting a compensatory mechanism may exist. Thus, as part of my dissertation, I characterized spinal motor nerve myelin phenotypes caused by having a mutation in $e r b b 3 b^{-/}$. My data demonstrated that Schwann cells failed to associate with motor axons in $e r b b 3 b^{-/-}$and, as a result, OPCs ectopically exited the CNS, associated with and myelinated spinal motor axons. This data was published by Smith et al. (2014) in a paper characterizing the novel CNS-derived peripheral myelinating glial cell population called MEP glia (Smith et al., 2014). Characterizing the spinal motor axon myelin defects in $e r b b 3 b^{--}$provided me with a unique and powerful model for assaying perineurial glia plasticity in response to ectopic OPCs during motor nerve assembly, maintenance and injury responses, described in this dissertation. 


\section{Perineurial glia: imperative for PNS development \& function}

The perineurium, initially described by Henle in 1841 and named by Key and Retzius in 1876 (Kucenas et al., 2008b; Thomas \& Olsson, 1984), is composed of concentric rings of flattened perineurial cells that ensheath myelinated axons (Figure 1-4A). Upon maturation, the perineurium serves as a mechanical and diffusion barrier to protect the nerve from its extracellular environment. While we know quite a bit about the perineurium ultrastructure and its capability to serve as the blood-nerve barrier upon maturation, emerging evidence demonstrates this glial cell population is a key mediator of motor nerve assembly (Binari et al., 2013; Clark et al., 2014; Kucenas et al., 2008b) and responding to axonal transection injuries (Lewis \& Kucenas, 2013; 2014).

\section{i. Debates surrounding perineurial origin}

The perineurium was previously thought to be derived from fibroblasts/mesenchymal cells that migrated to the perimeter of peripheral nerves (Bourne, 1968; Bunge et al., 1989; Conley et al., 1976; Kristensson \& Olsson, 1971; Pick et al., 1963; Shanthaveerappa \& Bourne, 1966). After migration, it was proposed that these mesenchymal cells would then undergo a transition to become epithelial cells and proceed to form the multiple layers of the perineurium (Bunge et al., 1989; Gamble, 1966; Gamble \& Breathnach, 1965; Halata et al., 1990; Haninec, 1988; Kristensson \& Olsson, 1971; Parmantier et al., 1999; Pick et al., 1963; Plessis et al., 1996). Shanthaveerappa and Bourne (1966) challenged the mesenchymal origin and proposed an ectodermal descent (Shanthaveerappa \& Bourne, 1966) and, recently, studies in Drosophila and zebrafish have confirmed that perineurial cells originate in the CNS as 
A

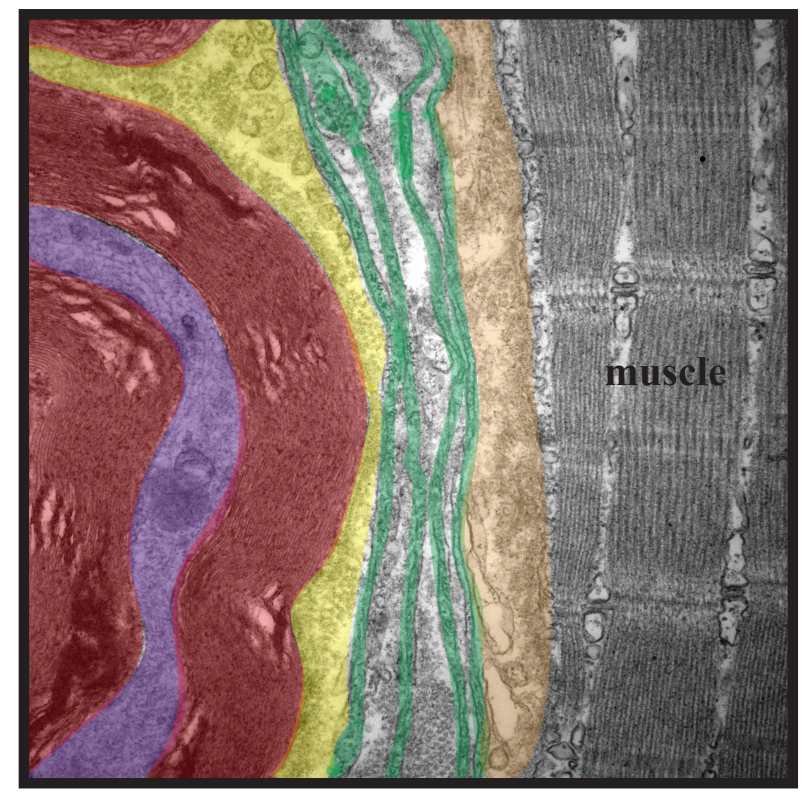

B

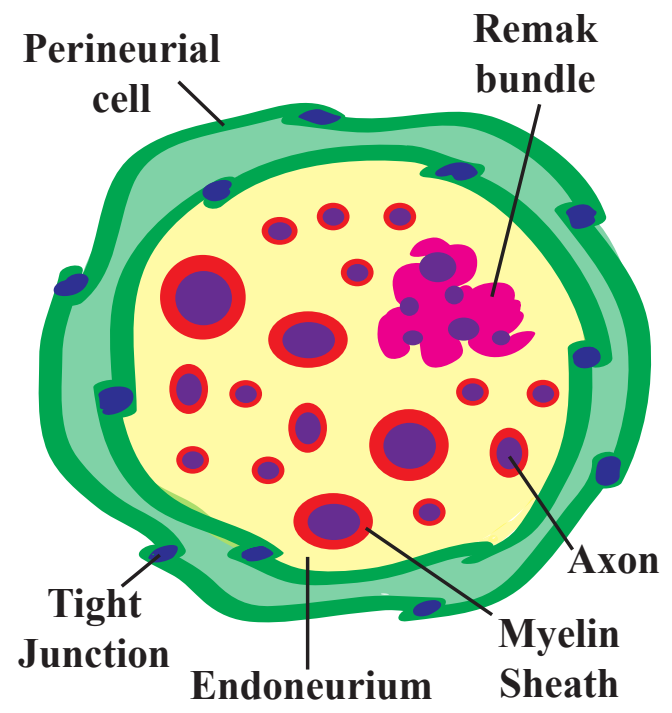

C

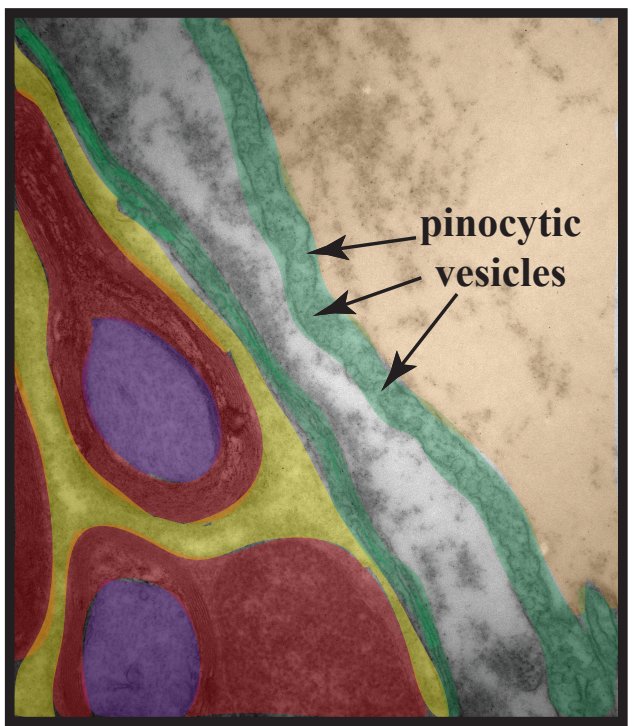

Figure 1-4. Perineurium ensheaths axons and their myelin sheaths. (A) Axon (purple) is myelinated (red) by peripheral myelinating glial cells, including MEP glia and Schwann cells. Endoneurium (yellow) surrounds myelinated axons and the perineurium (green) ensheaths myelinated axons to form a nerve fascicle. The epineurium (orange) encases several nerve fascicles to form the motor nerve. Magnification: 12k. (B) Perineurial glial cells form tight junctions (blue) with adjacent perineurial cells. Axon: purple, Myelin: red, Remak bundle: magenta, Endoneurium: yellow. (C) Perineurium contains pinocytic vesicles serving as a selective transport mechanism for the nerve fascicle. Axon: purple, Myelin: red, Endoneurium: yellow, Perineurium: green, Epineurium: orange. Magnification: 12k. 
glial cells (Kucenas et al., 2008b; Schmidt et al., 1997; Sepp et al., 2000; 2001).

In Drosophila, peripheral motor nerve ensheathing glia, which are functionally similar to vertebrate perineurial glia, arise in the ventral nerve cord and migrate from the lateral edges into the periphery to ensheath motor axons (Schmidt et al., 1997; Sepp et al., 2000; 2001). In the absence of peripheral glia, motor axons exit ectopically and have trouble pathfinding to their peripheral targets (Auld, 1999; Sepp et al., 2001). Kucenas et al. (2008) observed in zebrafish that the origin of vertebrate perineurial cells was also from the CNS (Kucenas et al., 2008b). In both mouse and zebrafish, perineurial glial cells arise from the p3 domain, migrate into the periphery via MEPs and eventually ensheath the myelinated motor nerve (Clark et al., 2014; Kucenas et al., 2008b).

A main reason for the difficulties in studying the origin of perineurial cells has been caused by a lack of markers to label this structure. A fairly recent discovery revealed the transcription factor $n k x 2.2 a$ as a marker for perineurial cells in zebrafish (Kucenas et al., 2008b) and mouse (Clark et al., 2014). Because of this, perineurial glial cells have emerged as key mediators involved in motor nerve assembly, maintenance and in response to axonal transection injuries described in greater detail below. In zebrafish, perineurial glia are visualized using the $\operatorname{Tg}(n k x 2.2 a$ :megfp $)$ transgenic reporter line in which $n k x 2.2 a$ regulatory sequences drive membrane-tethered EGFP in all $n k x 2.2 a^{+}$cells. This transgenic reporter line, coupled with in vivo time-lapse imaging, revealed that $n k x 2.2 a^{+}$cells were restricted to the $\mathrm{p} 3$ domain of the spinal cord in early development, migrated from the spinal cord via MEP transition zones at approximately $2 \mathrm{dpf}$ and, 
following proliferation and extension and retraction behaviors, ensheathed myelinated and unmyelinated peripheral axons, eventually differentiating into the mature perineurium (Kucenas et al., 2008b). In a later study, a $N k x 2.2$ :EGFP transgenic mouse reporter line confirmed that a subset of perineurial glial cells are CNS-derived in mammals (Clark et al., 2014). These studies not only clarified the origin debate surrounding the perineurum, but also produced useful tools for studying development, maintenance and injury response behaviors of this understudied glial cell population.

\section{ii. Nervous system barriers}

A recognized role of the perineurium is providing protection for the nerve. The mature perineurium extends from the MEP transition zone to the neuromuscular junction and, since it protects the peripheral nerve from its surrounding environment, is commonly referred to as the blood-nerve barrier (BNB) (Kucenas et al., 2008b; Shanthaveerappa \& Bourne, 1966). The mature perineurium is composed of many flattened perineurial cells that form tight junctions with adjacent perineurial cells (Figure 1-4B) (Akert et al., 1976). These tight junctions have been shown to express claudin-1, claudin-3, occludin and ZO1 in adult human sciatic nerves, and the expression of each tight junction marker follows a certain developmental pattern (Pummi et al., 2004). For example, claudin-1 expression begins early in development, claudin-3 and occludin expression starts later in development and ZO-1 appears to be expressed when the perineurium starts to mature, making ZO-1 a suitable marker for investigating perineurium differentiation (Pummi et al., 2004). 
Additionally, the perineurium consists of several layers with the total number dependent upon the size of the nerve fascicle as well as its vicinity to the CNS (Shanthaveerappa \& Bourne, 1966). Within mammals, such as monkey and humans, as many as 4-12 layers of concentric flattened cells may be found in nerve trunks (Shanthaveerappa \& Bourne, 1966) whereas zebrafish contain approximately 2-3 concentric layers per fascicle (Kucenas et al., 2008b). The perineurial layers are surrounded by organized basement membranes (also known as basal lamina) and collagen is present in between the layers. The load-bearing strength of the perineurium is due to the organization of the concentric cell layers and collagen, thus, the perineurium also serves as a mechanical barrier of protection for the nerve fascicle (Topp \& Boyd, 2006). In addition to mechanical protection, pinocytic vesicles are present throughout the perineurium (Figure 1-4C) serving as a selective transport mechanism and diffusion barrier for the nerve.

Since the mature perineurium serves as the BNB for peripheral nerves, this role is similar to the protection provided to structures of the CNS (i.e., brain and spinal cord) by the blood-brain barrier (BBB). The BBB of the CNS has been studied rather extensively in comparison to the BNB (Allt \& Lawrenson, 2000). The BBB and BNB provide a diffusion barrier of protection to shield the brain/spinal cord and peripheral nerves, respectively, from external insults in an effort to maintain homeostasis (Hawkins et al., 2006). However, the diffusion barrier of the mature perineurium is attributed to the tight junction formation by adjacent perineurial cells whereas the BBB is formed from tight junctions produced by capillary endothelial cells. These endothelial cells contain a basement membrane with embedded pericytes and are covered by collagen, fibronectin \& 
laminin. Additionally astrocytic processes surround the basement membranes; however, before astrocytes develop, some functional aspects of the BBB are already in existence, suggesting other cell populations may also be involved (Saunders et al., 2008). Recently, oligodendrocyte progenitor cells (OPCs) have been identified as promoting BBB integrity and may also influence the development of the BBB (Seo et al., 2014).

Similar to the BNB, tight junctions are an important feature of a functional BBB. The tight junctions formed by the BBB endothelial cells separate the capillary into a blood side (luminal) and a brain side (abluminal), thus, the transport of hydrophilic ions and molecules that cross into the brain must pass through both the luminal and abluminal sides (Hawkins et al., 2006). Regardless of the different cell populations comprising the $\mathrm{BBB}$ and $\mathrm{BNB}$, an important role of both barriers is to provide protection to their respective central and peripheral nervous system structures, and to do so requires that their barrier function capabilities is maintained.

\section{Peripheral glial-glial interactions in motor nerve assembly, maintenance and injury} responses

\section{i. Glial-glial interactions in development and nervous system maintenance}

Data from the Kucenas lab demonstrates perineurial glial cells are important for Schwann cell development and for ensuring proper positioning of motor axons (Binari et al., 2013; Clark et al., 2014; Kucenas et al., 2008b). When perineurial glia or $n k x 2.2 a$, the only known marker of perineurial glia expression is disrupted, motor axons exit the spinal cord ectopically and Schwann cells fail to form a myelin sheath, resulting in axonal 
defasciculation (Binari et al., 2013; Clark et al., 2014; Kucenas et al., 2008b). Reciprocally, factors derived from Schwann cells have also been shown to be important components for proper perineurial glia development and maturation, both in mouse and zebrafish. Although signaling mechanisms involved in perineurial development and maturation remain largely unknown, a key glial-glial mechanism that has been shown to be involved in promoting perineurial glial maturation in mice is the signaling between Schwann cell derived desert hedgehog (Dhh) and its receptor patched (Ptc), which is expressed by perineurial glial cells (Mirsky et al., 1999; Parmantier et al., 1999).

In mice, mutations in Schwann cell-derived factors, including desert hedgehog (Dhh), Sox10, and the G protein-coupled receptor Gpr126, result in perineurial maturation defects. Specifically, mice lacking Dhh have a thin, immature perineurium with leaky tight junctions, allowing for the infiltration of proteins, inflammatory cells and other molecules into the nerve (Parmantier et al., 1999). In $D h h^{-/-}$mice, the perineurium compartmentalizes the nerve fascicle into much smaller bundles, called mini-fascicles, as compared to wildtype nerves (Parmantier et al., 1999). The perineurium of Sox10 deficient mice appears very thin (Finzsch et al., 2010), and $\mathrm{Gpr} 126^{-/-}$mutant mice display perineurium defects similar to $D h h^{-/}$, including a disorganized perineurium with a patchy basal lamina and the formation of mini-fascicles (Monk et al., 2011). Interestingly, Sox10 has been shown to activate Dhh expression in Schwann cells (Kuspert et al., 2012) and Gpr126-- mice lack Dhh expression in their sciatic nerves (Monk et al., 2011), further supporting that a lack of Schwann cell derived Dhh impacts perineurial differentiation (Mirsky et al., 1999; Parmantier et al., 1999). This work also demonstrates that peripheral 
glial-glial interactions are imperative for motor nerve assembly and maintenance in mice.

Similarly, peripheral glial-glial interactions are important for spinal motor nerve assembly in zebrafish. Mont-blanc (mob) and mother-superior (mos) (mob/mos) mutant zebrafish are deficient for the transcription factors tfap2a and foxd3, respectively, and lack all neural crest derivatives including Schwann cells. In these mutants, perineurial glia extend processes, but fail to exit the spinal cord (Kucenas et al., 2009). Further supporting the importance of peripheral glial interactions in development.

Interestingly, $e r b b 3 b^{-/}$zebrafish lack peripheral myelinating glial cells, including MEP glia and Schwann cells (Lyons et al., 2005; Smith et al., 2014) and, because of the lack of peripheral myelinating glia, OPCs ectopically migrate into the periphery, associate with and myelinate spinal motor axons. This mutant zebrafish provides a unique model for assaying perineurial glial plasticity in nervous system development, maintenance and injury response behaviors because perineurial glial cells migrate from the spinal cord in $e r b b 3 b^{-/}$and ensheath motor nerves, despite the presence of ectopic OPCs, as described in greater detail in chapter 4. Furthermore, $e r b b 3 b^{-/-}$are homozygous viable and demonstrate relatively normal feeding and mating behaviors as compared to wildtype zebrafish (Honjo et al., 2011). Thus, as part of my dissertation, I used this mutant zebrafish model in which oligodendrocytes ectopically myelinate spinal motor axons to investigate the plasticity of perineurial glia during motor nerve development, differentiation and in response to injury. 


\section{ii. Glial-glial interactions in motor nerve injury and regeneration}

Peripheral nerves demonstrate a remarkably capacity for regeneration; however, functional recovery post-injury is often extremely poor (Allodi et al., 2012; Brushart et al., 1981; Hendry et al., 1986; Nguyen et al., 2002). Generally, nerve regeneration studies have focused on axons, Schwann cells and debris clearing macrophages during de- and re-generation processes. The role of perineurial glia in response to injury had remained poorly understood until a recent paper identified this ensheathing glial cell population as an active responder essential for nerve regeneration following an axonal transection injury (Lewis \& Kucenas, 2013; 2014). Using a laser transection assay in zebrafish, coupled with in vivo, time-lapse imaging, perineurial glia rapidly responded to nerve transections by extending membrane processes towards the injury site, phagocytizing debris and forming glial bridges that spanned the transection site allowing for axonal regrowth (Lewis \& Kucenas, 2014).

Since glial interactions have been identified as important processes involved in motor nerve assembly and maintenance, what happens to regeneration responses if peripheral myelinopathies cause the resident myelinating glial cell of the CNS, oligodendrocytes, to ectopically exit the CNS and myelinate peripheral axons? The erbb3b ${ }^{-/-}$zebrafish have ectopic oligodendrocytes that associate with and myelinate spinal motor axons and, interestingly, my data described in this dissertation demonstrates that perineurial glial cells migrate from the CNS, proliferate and perform extension and retraction behaviors to ensheath these myelinated axons in mutants. This model provides a unique environment 
in which a myelinating glial cell that normally resides within the poorly regenerative CNS is present within the typically regenerative PNS.

Previous work by Lewis et al. (2014) suggests that perineurial glia require the presence of peripheral myelinating glial cells, like MEP glia and Schwann cells, to perform all regenerative response behaviors (Lewis \& Kucenas, 2014). However, what happens if an oligodendrocyte is present? Are the perineurial glial cells capable of responding to injury? Or are certain behaviors altered or impaired? To address these questions, I collaborated with Wendy Lewis and she performed her in vivo laser axotomy assay using the $e r b b 3 b^{-/}$zebrafish. Intriguingly, the presence of ectopic oligodendrocytes, or centrally-derived myelin, altered certain perineurial glial response behaviors. To confirm that the impaired perineurial injury responses was due to the presence of the oligodendrocytes, and not because of the $e r b b 3 b^{-/}$mutation itself, $e r b b 3 b^{-/}$axonal tracts that lacked ectopic oligodendrocytes were fully transected and perineurial glial responses were assessed. Excitingly, perineurial cells were capable of responding to the injury by extending membrane, phagocytizing debris and forming glial bridges that spanned the transection site, upon injurying tracts that lacked ectopic oligodendrocytes. Thus, this regeneration work further supports that intricate glial-glial interactions are necessary for injury response behaviors, as well as elegantly reemphasizes the role perineurial glia play as mediators of regeneration. 


\section{CNS Demyelination: Oligodendrocytes under attack}

The perineurial glial plasticity project revealed a peripheral myelinopathy mutant, $e r b b 3 b^{-/}$zebrafish, in which the resident myelinating glial cell population of the CNS, OPCs, ectopically exit via MEP transition zones to associate with and myelinate spinal motor axons. Intriguingly, these ectopically-located oligodendrocytes demonstrated remarkable resilience to their non-native environment and were capable of myelinating and surviving in the PNS. This resiliency, coupled with the beautifully dynamic behaviors of this myelinating glial cell, led me to another project: investigating the responses and behaviors of oligodendrocytes following exposure to a demyelinating insult in the CNS. To our knowledge, only generalized demyelination models, and no focal demyelination assay, using zebrafish larvae existed prior to my work. Thus, I created and characterized a focal drug-induced demyelination model in zebrafish to visualize myelin breakdown and repair in vivo.

\section{i. Myelin breakdown in the CNS}

As described above, oligodendrocytes are the resident myelinating glial cells of the CNS. Unfortunately, demyelinating disorders like multiple sclerosis (MS) target oligodendrocytes, destroying the myelin sheath. This loss of myelin has devastating consequences. Axons become exposed and vulnerable to their environment, resulting in axonal degeneration. Myelin loss also leads to impairment of action potential propagation, resulting in motor and sensory defects that eventually lead to disability and a life dependent upon walking aids and assistance. 
Although MS is commonly thought of as a neuroinflammatory disease characterized by the immune system inappropriately attacking the insulating myelin sheath surrounding axons of the CNS, the primary etiology or initial trigger that causes MS remains unknown. Additionally, how demyelination occurs and the processes involved with remyelinating naked axons within a demyelinated lesion are not well understood. Mammalian models commonly used to study MS and patient samples are not amenable for visualizing processes underlying myelin destruction and repair in vivo. Because of this, there may be unappreciated cellular events involved in demyelination and subsequent remyelination, and a novel model that allows for in vivo visualization of the temporal dynamics and cellular interactions underlying these processes is needed.

\section{ii. Animal models of $M S$}

MS is an extremely heterogeneous disease, ranging from clinical pathology, symptoms, lesion location and response to therapy (Lassmann et al., 2001; Lucchinetti et al., 2000; Stys et al., 2012). Because of this heterogeneity and the unknown etiology that triggers MS, it's important to have various animal models that recapitulate the disease or its associated symptoms. Mammalian systems have been instrumental for studying MS and commonly used models include experimental autoimmune encephalomyelitis (EAE) and toxin-induced demyelination.

EAE has provided a better understanding about the underlying autoimmune neuroinflammatory response that is characteristic of MS (Denic et al., 2011; Ransohoff, 2012). EAE is typically induced in mice by injecting synthetic myelin peptides. 
Following injection, the immune system becomes activated and primed against the myelin peptide and antigen-specific T-cells infiltrate into the CNS and attack myelin (Denic et al., 2011). EAE lesions have been described as resembling MS pathology (Ransohoff, 2012; Waksman \& Adams, 1962) and the neuroinflammatory aspects of this model have been beneficial for investigating the inflammatory response and immunemediated damage associated with MS pathology (Denic et al., 2011). However, the C57B1/6 mouse strain that is most frequently used for inducing EAE produces a monophasic disease course, and therefore, relapses and remyelination are lacking (Ransohoff, 2012). Furthermore, the model has proven inconsistent for predicting the success of therapeutic strategies in MS patients (Denic et al., 2011), thus, additional animal models are imperative for investigating compounds that reduce demyelination or promote remyelination.

Toxin-induced demyelination models have been valuable for investigating both demyelination caused by a direct effect on the oligodendrocyte and processes underlying remyelination (Blakemore \& Franklin, 2008; Blakemore et al., 1977; Foote \& Blakemore, 2005; Ransohoff, 2012). Toxin-induced demyelination models demonstrate a relatively predictable time-course for events underlying demyelination and remyelination, providing an advantage of separating processes associated with myelin destruction and subsequent repair (Miller \& Fyffe-Maricich, 2010; Ransohoff, 2012). Compounds that are commonly used to induce demyelination include the copper chelator cuprizone and the membrane solubilizing agent lysophosphatidylcholine, commonly referred to as lysolecithin (Ransohoff, 2012). 
In the cuprizone model, mice receive this copper chelator in their diet and demyelination typically occurs in the corpus callosum, internal capsule, anterior commissure and cerebellar peduncles (Blakemore \& Franklin, 2008). Remyelination of the affected white matter tracts is initiated upon removal of cuprizone and extensive myelin repair is apparent within approximately 3 or 4 weeks post-removal (Matsushima \& Morell, 2001). Although cuprizone reliably initiates demyelination without damaging other CNS cell types, the factors that make oligodendrocytes especially vulnerable to this toxin are not well understood (Denic et al., 2011). It has been proposed that the high metabolic demands by oligodendrocytes cannot be fulfilled when copper is deficient (Denic et al., 2011), resulting in mitochondrial dysfunction (Matsushima \& Morell, 2001). Regardless of the mechanism that makes oligodendrocytes particular vulnerable to cuprizone, an advantage to this model is it reliably and reproducibly results in demyelination and remyelination upon removal of the toxin. This is particularly beneficial for investigating processes associated with de- and re-myelination without the complications of a neuroinflammatory response.

In addition to cuprizone, another toxin that has been used to induce demyelination is lysolecithin. Lysolecithin is a membrane solubilizing agent and activates phospholipase A2 (Denic et al., 2011). Lysolecithin is administered via microinjection into the CNS, typically within the white matter tracts of the spinal cord (Blakemore \& Franklin, 2008; Denic et al., 2011; Ransohoff, 2012). Lysolecithin is especially toxic for myelin and oligodendrocytes (Blakemore \& Franklin, 2008; Keirstead \& Blakemore, 1997; McKay et al., 1998) and typically spares axons, although some axonal loss does occur within the 
injection site (Blakemore \& Franklin, 2008). It has been proposed that demyelination is caused by the solubilization of the myelin sheath by this detergent (Denic et al., 2011; Hall, 1972). However, the exact mechanisms resulting in demyelination following exposure to lysolecithin are not known. Following injection of lysolecithin, there are consistent phenotypes that have been described, including: myelin breakdown, loss of oligodendrocytes, infiltration by professional phagocytes (microglia / macrophages), OPC proliferation and recruitment to the lesion site and remyelination (Blakemore et al., 1977; Blakemore \& Franklin, 2008; Foster et al., 1980; Jeffery \& Blakemore, 1995; Lachapelle et al., 2005; Ousman \& David, 2000). Similar to the cuprizone model, demyelination induced by lysolecithin is not initially caused by an autoimmune attack on the myelin sheath, and, because of this, the toxin models have great potential for screening therapeutic compounds that inhibit myelin breakdown or accelerate its repair.

A limitation to mammalian models of MS is the inability to visualize de- and remyelination in vivo. Thus, data from these models only captures a snap-shot in time. Zebrafish are amenable to investigating myelin breakdown and repair in a living vertebrate organism. Currently, a generalized demyelination model exists in zebrafish that takes advantage of the ease of drug submersion combined with tissue-specific ablation by the bacterial nitroreductase gene. However, a major limitation of this model is the inability to create focal lesions, which are a hallmark of MS pathology (Chung et al., 2013; Fang et al., 2015). Lysolecithin placed on a gel foam has also been applied directly to the adult zebrafish optic nerve to induce demyelination (Münzel et al., 2014). Although this zebrafish model recapitulates phenotypes reported in lysolecithin-induced 
demyelination models in mammals, data from this model is from fixed tissue and, once again, provides information from a snap-shot in time.

Interestingly, the effects of lysolecithin injections in the zebrafish spinal cord have not been described. Thus, I injected lysolecithin into the spinal cord of zebrafish larvae and visualized myelin breakdown in vivo. Furthermore, this model recapitulates phenotypes reported in mammalian lysolecithin induced demyelination models, including altered numbers and behaviors of Sox $10^{+}$oligodendrocytes, microglia / macrophage infiltration into the lesion area and myelin membrane changes. Excitingly, zebrafish exposed to the anti-muscarinic agent Clemastine fumarate, that has previously been shown to enhance remyelination kinetics in mice, had reduced myelin membrane changes post-lysolecithin injection. This dissertation will also present data characterizing this novel model, demonstrating responses by OPCs and oligodendrocytes following a myelinopathy induced by a demyelinating insult.

\section{Zebrafish: a powerful model system with unique advantages}

In this manuscript, I present data using zebrafish (Danio rerio) (Figure 1-1A) as a model to study glial-glial interactions during spinal motor nerve assembly and for investigating glial responses following a demyelinating insult. Zebrafish offer unique advantages for studying glial cells during development and in response to injury. Zebrafish embryos are optically transparent allowing for direct observation of cellular processes within an intact vertebrate system. Furthermore, development occurs externally, making it an ideal model for investigating developmental processes in vivo. This optical transparency, coupled 
with the plethora of transgenic lines available that fluorescently label different cell populations, allow us to visualize cell behaviors, interactions and responses in a living vertebrate, in real time.

Conventionally, invertebrate and vertebrate model systems have been used to study the role of glial cells in nervous system development and in response to myelinopathies. However, murine and avian vertebrate models lack the ability to directly observe cellular behaviors within the majority of the nervous system in vivo, thus, providing data simply from a snapshot in time. Invertebrate models, such as Drosophila melanogaster and Caenorhabditis elegans (C. elegans), do offer a wide variety of genetic manipulations for investigating glial cells within a simpler system, but vertebrate and invertebrate glia differ significantly. Zebrafish share developmental features similar to avian and murine models, including remarkable homology to humans as there is at least one zebrafish orthologue for approximately $70 \%$ of human genes (Howe et al., 2013). Thus, allowing for inferences regarding glial interactions and responses to be made for other vertebrate systems.

My data provides a detailed demonstration about the importance of glial interactions during development and in response to myelinopathies and further supports that zebrafish are an ideal model for investigating glial development, injury responses and maintenance behaviors in vivo. Specifically, my data demonstrates that certain developmental and injury response behaviors by perineurial glia are remarkably plastic despite a peripheral neuropathy in which oligodendrocytes ectopically myelinate spinal motor axons, and 
oligodendrocyte demyelination can be visualized in real-time, in a living vertebrate. In Chapter 3, I describe a technique that allows us to easily identify regions within the nervous system to investigate glial cell ultrastructure in the adult zebrafish nervous system. This modified transmission electron microscopy protocol is not only useful for studying glial cell populations associated with adult spinal motor nerves, but can also be adapted to study glial cells within specific regions of the CNS, as well as during various time-points including development and maturation. In Chapter 4, I investigate perineurial plasticity in response to a peripheral myelinopathy using in vivo imaging, immunohistochemistry and the TEM protocol described in Chapter 3. My data demonstrates that although perineurial glial migration, proliferation and extension behaviors are comparable to wildtype during development, certain injury responses are altered and, ultimately, the perineurial barrier function is compromised. Based on these results, this work further supports the importance of peripheral glial-glial interactions in forming and maintaining a functional spinal motor nerve. In Chapter 5, my investigation of oligodendrocytes shifted back to their resident environment of the CNS as I created and characterized a novel focal demyelination model in zebrafish. This model allows for visualizing myelin breakdown and repair in vivo and may be useful as a potential drugscreening assay for identifying novel therapeutic compounds for treating demyelinating disorders like MS. Overall, this work has provided a better understanding about the plasticity of glial cells during nervous system development and in response to myelinopathies. 


\section{Chapter 2}

\section{Materials and Methods}

\section{Fish husbandry}

All animal studies were approved by the University of Virginia Institutional Animal Care and Use Committee. Zebrafish strains used in this study included AB (RRID:ZIRC_ZL1), $\operatorname{Tg}(n k x 2.2 a: m e g f p)^{v u 17}$ (RRID:ZFIN_ZDB-ALT-080321-1) (Kirby et al., 2006; Kucenas et al., 2008b), $\operatorname{Tg}(\operatorname{sox} 10(7.2): m r f p)^{v u 234}$ (RRID:ZFIN_ZDB-ALT080321-3) (Kucenas et al., 2008b), Tg(olig2:dsred2) ${ }^{\text {vu19 }}$ (RRID:ZFIN_ZDB-ALT080321-2) (Kucenas et al., 2008b), Tg(NBT:DsRed) (RRID:ZFIN_ZDB-ALT-081027-2) (Peri \& Nüsslein-Volhard, 2008), $\quad e r b b 3 b^{\text {st48-/- }}$ (RRID:ZFIN_ZDB-ALT-050512-6) (Lyons et al., 2005), $n r g 1^{z 26-/-}$ (RRID:ZFIN_ZDB-GENO-120309-6) (Perlin et al., 2011) Tg(mbp:EGFP-CAAX) (RRID:ZFIN_ZDB-ALT-120103-2) (Almeida et al., 2011), Tg(cntnlb:mCherry) (RRID:ZFIN_ZDB-GENO-140610-7) (Czopka et al., 2013), Tg(mpegl:mCherry) (RRID:ZFIN_ZDB-ALT-120117-2) (Ellett et al., 2011), $\operatorname{Tg}$ (mpeg1:EGFP) (RRID:ZFIN_ZDB-GENO-120402-4) (Ellett et al., 2011), $\operatorname{Tg}$ (sox10:nls-eos) (RRID:ZFIN_ZDB-ALT-110721-2) (McGraw et al., 2012), Tg(sox10:gal4-vp16) (RRID:ZFIN_ZDB-ALT-150803-2) (Chung et al., 2013) and $\operatorname{Tg}\left(\right.$ UAS:Lifeact-GFP) ${ }^{\text {mu271 }}$ (RRID:ZFIN_ZDB-GENO-130624-2) (Helker et al., 2013). Table 1 describes the expression and abbreviations of all transgenic and mutant lines used in this dissertation. Embryos were produced by pairwise matings, raised at $28.5^{\circ} \mathrm{C}$ in egg water, staged according to hours or days post fertilization (hpf and dpf, respectively) and embryos of either sex were used for all experiments (Kimmel et al., 1995). Embryos used 
Table 2-1. Descriptions and abbreviations of transgenic and mutant lines

\begin{tabular}{|c|c|c|}
\hline Transgene name & Abbreviation & Description of expression \\
\hline $\operatorname{Tg}(m b p: E G F P-C A A X)$ & $m b p: E G F P-C A A X$ & $\begin{array}{l}\text { Membrane EGFP in } m b p^{+} \text {cells } \\
\text { (oligodendrocytes) }\end{array}$ \\
\hline Tg(mpeg1:mCherry) & mpegl:mCherry & $\begin{array}{l}\text { Cytosolic mCherry in } \text { mpeg }^{+} \text {cells } \\
\text { (macrophages, microglia) }\end{array}$ \\
\hline $\operatorname{Tg}(m p e g 1: E G F P)$ & mpegl:EGFP & $\begin{array}{l}\text { Cytosolic EGFP in } \text { mpeg }^{+} \text {cells } \\
\text { (macrophages, microglia) }\end{array}$ \\
\hline $\operatorname{Tg}(\operatorname{sox} 10: n l s-E o s)^{w 18}$ & sox 10:nls-eos & $\begin{array}{l}\text { Nuclear localized Eos in sox } 10^{+} \\
\text {cells (OPCs, oligodendrocytes) }\end{array}$ \\
\hline $\operatorname{Tg}(\operatorname{sox} 10(7.2): m r f p)^{v u 234}$ & sox 10:mrfp & $\begin{array}{l}\text { Membrane RFP in sox } 10^{+} \text {cells } \\
\text { (Schwann cells, MEP glia, OPCs, } \\
\text { oligodendrocytes) }\end{array}$ \\
\hline $\operatorname{Tg}(\operatorname{sox} 10:$ Gal4-vp 16) & sox 10:Gal4 & $\begin{array}{l}\text { Gal } 4 \text { specifically expressed in } \\
\text { sox } 10^{+} \text {cells (OPCs, } \\
\text { oligodendrocytes) }\end{array}$ \\
\hline $\operatorname{Tg}(\text { olig2:dsred } 2)^{v u 19}$ & olig2:dsred & $\begin{array}{l}\text { Cytosolic DsRed in olig2 } 2^{+} \text {cells } \\
\text { (motor neurons, axons, OPCs, } \\
\text { oligodendrocytes) }\end{array}$ \\
\hline $\operatorname{Tg}(U A S: \text { Lifeact-GFP })^{m u 271}$ & Lifeact-GFP & $\begin{array}{l}\text { UAS promoter driving Lifeact-GFP } \\
\text { to label filamentous actin (F-actin) }\end{array}$ \\
\hline $\operatorname{Tg}(n k x 2.2 a: m e g f p)^{v u 1}$ & $n k x 2.2 a: m e g f p$ & $\begin{array}{l}\text { Membrane EGFP in } n k x 2.2 a^{+} \text {cells } \\
\text { (perineurial glia, floorplate, OPCs, } \\
\text { oligodendrocyte) }\end{array}$ \\
\hline $\operatorname{Tg}($ cntn 1 b:mCherry) & cntnlb:mCherry & $\begin{array}{l}\text { Cytosolic mCherry in mature } \\
\text { neurons and axons }\end{array}$ \\
\hline $\operatorname{Tg}(N B T: D s R e d)$ & nbt:dsred & $\begin{array}{l}\text { Cytosolic DsRed in } n b t^{+} \text {cells } \\
\text { (neurons, axons) }\end{array}$ \\
\hline$e r b b 3 b^{s t 48}$ & erbb3 & $\begin{array}{l}\text { Premature stop codon that truncates } \\
\text { protein in extracellular domain }\end{array}$ \\
\hline$n r g 1^{z 26}$ & $n r g 1$ & $\begin{array}{l}\text { T-to-C transition converts cysteine } \\
\text { in cysteine rich domain to arginine } \\
\text { and likely disrupts protein folding } \\
\text { or localization }\end{array}$ \\
\hline
\end{tabular}

All lines were stable, germline transgenics or mutants. Only cell types pertinent to this study are listed for each transgene. 
for immunohistochemistry, microscopy and lysolecithin injections were treated with $0.003 \%$ phenylthiourea (PTU) in egg water to inhibit pigmentation. Adult zebrafish used for transmission electron microscopy were approximately 8 months old and were euthanized by immersion in $0.4 \%$ Tricaine.

\section{Genotyping}

Genomic DNA samples were scored for the $e r b b 3 b^{s t 48}$ mutation by digesting individual PCR products, amplified using primers 5'-GTGTCTGTTGCAGAATGGTAAA-3' and 5'-AGAAAACCCTGACTGGACCA-3', with the Hpy8I (MjalV) restriction enzyme (Thermo Scientific, ER1571) (Lyons et al., 2005; Monk et al., 2009). For adult zebrafish, fin tissue was placed in $75 \mu \mathrm{l}$ of Lysis Reagent ( $25 \mathrm{mM} \mathrm{NaOH} / 0.2 \mathrm{~mm}$ EDTA) and stored on ice at approximately $15^{\circ} \mathrm{C}$ until digested using a PCR machine. Samples were digested as per the HotSHOT digest protocol (Lyons et al., 2008; Meeker et al., 2007): $95^{\circ} \mathrm{C}$ for 30 minutes, sample cooled to $4^{\circ} \mathrm{C}, 75 \mu \mathrm{l}$ of Neutralization Buffer $(40 \mathrm{mM}$ Tris $\mathrm{HCl}$ ) added, samples were spun-down and $1 \mu \mathrm{l}$ of DNA lysate was used for genotyping PCR reaction. Genomic DNA samples were evaluated for the erbb $3 b^{s t 48}$ mutation as described above.

\section{In vivo imaging}

At $24 \mathrm{hpf}$, all embryos used for live imaging were transferred to egg water containing PTU to inhibit pigment formation. At specified stages, embryos and larvae were anesthetized using 3-aminobenzoic acid ester (Tricaine), immersed in $0.8 \%$ low-melting point agarose and mounted on their sides in glass-bottomed $35 \mathrm{~mm}$ Petri dishes (Electron 
Microscopy Sciences). Images were captured using either a 40X (numerical aperture 1.2) or a $63 \mathrm{X}$ (numerical aperture 1.2) water-immersion objective mounted on a motorized Zeiss AxioObserver Z1 microscope equipped with a Quorum WaveFX-X1 spinning disc confocal system (Quorum Technologies). For time-lapse imaging, $z$-stacks were collected at specified time points and three-dimensional datasets were compiled using MPEG-4 video compression at 10 frames per second and exported to QuickTime (Apple) to create movies. Image adjustments were limited to contrast enhancement and level settings using MetaMorph software (RRID:SCR_002368), Adobe Photoshop (RRID:SCR_014199) and ImageJ (RRID:SCR_003070).

\section{Immunohistochemistry}

\section{i. Whole mount}

Larvae were fixed in AB Fix (4\% PFA, 0.1\% Triton-X 100, 1X PBS) for either $3 \mathrm{~h}$ at 23 ${ }^{\circ} \mathrm{C}$ or overnight at $4{ }^{\circ} \mathrm{C}$, followed by a 5 min wash with PBSTx (1\% Triton X-100, $1 \mathrm{X}$ PBS), a 5 min wash with DWTx (1\% Triton X-100 in distilled water), a 5 min wash with acetone at $23{ }^{\circ} \mathrm{C}$, a 10 min wash with acetone at $-20{ }^{\circ} \mathrm{C}$ and three 5 min washes with PBSTx. Larvae were preblocked in 5\% goat serum/PBSTx for at least $1 \mathrm{~h}$ and incubated in primary antibody for $1 \mathrm{~h}$ at $23{ }^{\circ} \mathrm{C}$ and overnight at $4{ }^{\circ} \mathrm{C}$. The primary antibodies used include: mouse anti-acetylated tubulin (1:5000, Sigma Cat\# T7451, RRID:AB_609894), a rabbit antibody to Sox10 (1:5000, Thermo Fisher Scientific Cat\# Kucenaslab_001, RRID:AB_2637056) (Binari et al., 2013), a mouse antibody to zonula occludens-1 (ZO1; 1:200, Invitrogen Cat\# 33-9100, RRID:AB_2533147) and a rabbit antibody to antiphospho-Smad3 (1:175, Abcam Cat\# ab52903, RRID:AB_882596) (Casari et al., 2014). 
Larvae were washed extensively with $1 \mathrm{X}$ PBSTx and stored in $50 \%$ glycerol-PBS at $4{ }^{\circ} \mathrm{C}$ until imaging. Larvae were mounted on their sides in $0.8 \%$ low-melting point agarose on glass-bottomed $35 \mathrm{~mm}$ Petri dishes and imaged using the confocal microscope described above. Image adjustments were limited to contrast enhancement and level settings using MetaMorph software, Adobe Photoshop and ImageJ.

\section{ii. Sectioned tissue}

For immunohistochemistry on sections, adults $>3$ months old were fixed in $\mathrm{AB}$ Fix as described above for 3 days at $23{ }^{\circ} \mathrm{C}$ and $4{ }^{\circ} \mathrm{C}$ overnight. The anterior and posterior ends were removed, such that only approximately a $0.5 \mathrm{~cm}$ region of the trunk remained. Trunk portions were embedded in $1.5 \%$ agar $/ 30 \%$ sucrose and frozen in 2-methylbutane chilled by immersion in liquid nitrogen. Coronal sections $(50 \mu \mathrm{m}$ for adults) were collected on microscope slides using a cryostat microtome. Sections were hydrated in $1 \mathrm{X}$ PBS for $60 \mathrm{~min}$ at $23{ }^{\circ} \mathrm{C}$ and preblocked in $2 \%$ goat serum/BSA/1X PBS for $30 \mathrm{~min}$. Sections were incubated in primary antibody overnight at $4{ }^{\circ} \mathrm{C}$. Sections were washed extensively with $1 \mathrm{X}$ PBS, incubated for $3 \mathrm{~h}$ at $23{ }^{\circ} \mathrm{C}$ with Alexa Fluor 647 goat antimouse (1:600, Invitrogen) and washed with 1X PBS for $30 \mathrm{~min}$. Sections were mounted in Vectashield (Vector Laboratories) and imaged using the confocal microscope described above. Image adjustments were limited to contrast enhancement and level settings using Metamorph software, Adobe Photoshop and ImageJ. Fluorescence intensity measurements were collected using ImageJ. Six circular regions of interest (ROI) were selected within $n k x 2.2 a^{+} / \mathrm{ZO}-1^{+}$regions of the perineurium and the area, integrated density and mean gray value were measured for each ROI. One background 
measurement was selected per image in an area that contained no fluorescence. The mean gray value was calculated for background ROIs and used to calculate the corrected total fluorescence for each individual ROI using the following equation: Integrated density (ROI area $\mathrm{x}$ mean fluorescence of background images). The corrected total fluorescence for each ROI was used to determine statistical significance between erbb3 $3 b^{-/}$and wildtype labeling using GraphPad Prism software (RRID:SCR_002798).

\section{Transmission electron microscopy}

Adult wildtype and $e r b b 3 b^{-/}$zebrafish (approximately 8 months old) were euthanized with Tricaine, fin tissue was collected for genotyping and the trunk region was dissected and placed in fixative: $2 \%$ gluteraldehyde/ $4 \%$ PFA in $0.1 \mathrm{M}$ sodium cacodylate. Adult zebrafish trunk samples were prepared for electron microscopy using microwave fixation (Panasonic Model \#NNSD967S) and were kept on ice at $15{ }^{\circ} \mathrm{C}$ to prevent sample heating. Samples were then kept in fixative for at least $2 \mathrm{~h}$ at $23{ }^{\circ} \mathrm{C}$. Samples were stored in $2.5 \%$ gluteraldehyde in $0.1 \mathrm{M}$ sodium cacodylate at $4{ }^{\circ} \mathrm{C}$ until genotyping results were available. Upon confirmation of genotype, trunk samples were mounted in $1.75 \%$ gluteraldehyde in gelatin/albumin (Gelatin from porcine skin, Sigma Type A G1890; Bovine Serum Albumin Sigma A3912) embedding medium and $200 \mu \mathrm{m}$ transverse sections were cut using a Leica VT1000S vibrating blade microtome. The sections were then washed in $0.1 \mathrm{M}$ sodium cacodylate, 2 times at $3 \mathrm{~min}$ per wash. The sections were removed from the gelatin/albumin embedding medium and transferred to a glass scint vial for secondary fixation. $1 \%$ osmium $(\mathrm{OsO} 4)$ in $0.1 \mathrm{M}$ sodium cacodylate was added to the sections and incubated at approximately $23{ }^{\circ} \mathrm{C}$ for approximately $1 \mathrm{~h}$. The $1 \%$ OsO4 
in $0.1 \mathrm{M}$ sodium cacodylate was extracted and the samples were rinsed 3 times, 3 min per wash, in $0.1 \mathrm{M}$ sodium cacodylate. The samples were dehydrated as follows: $50 \%$ ethanol for $3 \mathrm{~min}, 4 \%$ uranyl acetate in $70 \%$ ethanol for at least $1 \mathrm{~h}, 70 \%$ ethanol for $1 \mathrm{~min}, 90 \%$ ethanol for $5 \mathrm{~min}, 100 \%$ ethanol for $5 \mathrm{~min}, 2$ times. Samples were rinsed in acetone 3 times, 2 min per rinse and stored in a 1:1 EPON:acetone mixture at approximately $23{ }^{\circ} \mathrm{C}$ overnight. The 1:1 EPON:acetone mixture was replaced with full EPON and samples were incubated at approximately $23^{\circ} \mathrm{C}$ overnight. Samples used for coronal cross sections were embedded in full EPON and the EPON was polymerized in a $60{ }^{\circ} \mathrm{C}$ oven overnight. Ultrathin sections $(70 \mathrm{~nm})$ were obtained on a Leica UCT ultramicrotome, transferred to copper grids and counterstained with uranyl acetate. Coronal cross sections were visualized using a Zeiss Sigma HD variable pressure scanning electron microscope with a STEM detector. Images were taken at 25000x magnification for Figure 4-2F and 4-2G (top), 15000x magnification for Figure 4-7H and 5000x magnification for Figure 4-4C. For transverse sections, sections were flat embedded in between two ACLAR $®$ embedding sheets using full EPON and put in a $60{ }^{\circ} \mathrm{C}$ oven overnight. Samples were excised from the ACLAR ${ }^{\circledR}$ embedding sheets and re-embedded in BEEM® embedding capsules. The embedding capsules were placed in a $60{ }^{\circ} \mathrm{C}$ oven overnight to polymerize the EPON. Following polymerization, the samples were removed from the capsule and were trimmed for ultrathin sectioning. Ultrathin transverse sections $(70 \mathrm{~nm})$ were obtained on a Leica Ultracut UCT Ultramicrotome, transferred to copper grids and were visualized using a JEOL 1010 Transmission electron microscope. Images for Figures 4$2 \mathrm{G}$ (bottom), 4-4D and 4-4E were taken at 1200x magnification with a 16 megapixel CCD camera (SIA-Scientific Instruments and Applications). 


\section{i. Transmission electron microscopy equipment and materials}

- $0.4 \%$ Tricaine-S (MS 222; Western Chemical Inc) in egg water

- Paraformaldehyde (Sigma, P6148)

- 50\% EM Grade Aqueous Gluteraldehyde, (Electron Microscopy Sciences, Cat. \#16316-10)

- Sodium Cacodylate Trihydrate (Sigma C0250)

- $50 \mathrm{~mL}$ Polypropylene Conical Tubes (Falcon, REF\# 352098)

- Untreated 24-Well Polypropylene Flat Bottom Microplates (Evergreen Scientific \#290-8324-03F)

- Untreated Polypropylene Lids for 24-Well Plates (Evergreen Scientific \#2908126-03L)

- Microwave (Panasonic Model \#NNSD967S)

- Gelatin from porcine skin, Type A (Sigma G1890)

- Bovine Serum Albumin (Sigma A3912)

- Peel-A-Way® disposable embedding molds (T-8) (Polysciences Cat\# 18985)

- Leica VT1000S vibrating blade microtome

- Leica EM UC7 or Leica Ultracut UCT ultramicrotome

- Forceps, Student Dumont \#5 Inox (Fine Science Tools, Item \#91150-20)

- Paintbrush (Simply Simmons Bristle Round Brush, Size 1)

- $20 \mathrm{~mL}$ Borosilicate Glass Scintillation Vials with White Polypropylene Caps (Fisherbrand, Article \# FS74504-20)

- Ethanol (Ethyl alcohol, pure, 200 proof, ACS reagent) (Sigma 459844) 
- Acetone, Glass Distilled, CAS \#67-67-1 (Electron Microscopy Sciences, Cat. \# 10015)

- $4 \%$ Osmium tetraoxide solution (OsO4) (Sigma, 75632)

- Small Flat Embedding Mold (PELCO ${ }^{\circledR} 106$, Ted Pella, Inc.)

- ACLAR embedding film, 7.8 mil Thickness (Electron Microscopy Sciences, Cat. \#50425-25)

- BEEM ${ }^{\circledR}$ Embedding Capsules, Size: 00 (Electron Microscopy Sciences, Cat. \#70010-B)

- EPON

- Uranyl acetate in ethanol

- Copper grids (Electron Microscopy Sciences Cat. \#EMS G200-Cu)

- Anti-Acetylated Tubulin Antibody (Sigma, Cat. \#T7451, RRID: AB_609894)

- Black Gold II Myelin Staining Kit (Histo-Chem Inc., Product \#IBGII)

- Zeiss Axio Zoom.V16 fluorescence stereo zoom microscope

- Objective stage micrometer $(1.0 \mathrm{~mm} \times 0.01 \mathrm{~mm})$

- JEOL 1010 transmission electron microscope or a Zeiss Sigma HD variable pressure scanning electron microscope with a STEM detector

\section{Nerve transection}

Nerve transections were performed using a MicroPoint Laser (Andor Technology) as previously published (Banerjee et al., 2013; Binari et al., 2013; Lewis \& Kucenas, 2013; 2014; Rosenberg et al., 2012) attached to a spinning disk confocal system (Quorum 
Technologies) consisting of a nitrogen-pumped dye laser (wavelength $435 \mathrm{~nm}$ ) controlled by MetaMorph Version 7.7. Ablation laser settings ranged from power 15 to 20 depending on the age of the larvae and nerve position. One or two motor nerves per larva in hemisegments $10-16$ were transected in all experiments. To transect nerves, a thin elliptical ROI was drawn digitally in MetaMorph over the image of the nerve, and the nerve was laser pulsed precisely within the ROI until the nerve appeared transected, whereby fluorescence did not refill the ROI in $>10$ seconds. Successful transections with this protocol were independently confirmed by identifying the presence of axonal degeneration in transgenic lines that label motor axons (data not shown). In vivo imaging of transected nerves was performed as described above.

\section{Perineurium barrier integrity assay}

Adult sox 10:mrfp and sox 10:mrfp; $\operatorname{erbb}^{-/-}$zebrafish, approximately 8 months old, were anesthetized using Tricaine. Dextran conjugated to Alexa Fluor ${ }^{\circledR} 647$ (10,000 MW, anionic, fixable; Life Technologies Cat\# D22914) was diluted to a $2 \%$ working stock concentration, loaded into a micropipette glass capillary (World Precision Instruments, Inc. TW100F-4) and injected into the muscle of the trunk, with at least 3 muscle injection sites per fish. Following injection of the fluorescent dye, the zebrafish were allowed to recover. At 1.5 hours post injection (hpi), the zebrafish were euthanized using Tricaine and the anterior and posterior ends were removed and the dissected trunk region was placed in $4 \%$ PFA for 3 days at $23{ }^{\circ} \mathrm{C}$ and at $4{ }^{\circ} \mathrm{C}$ overnight. Trunk portions were embedded in $1.5 \%$ agar $/ 30 \%$ sucrose and frozen in 2-methylbutane chilled by immersion in liquid nitrogen. Coronal sections $(50 \mu \mathrm{m})$ were collected on microscope slides using a 
cryostat microtome. Sections were mounted in Vectashield (Vector Laboratories) and imaged using the confocal microscope described above. Image adjustments were limited to contrast enhancement and level settings using Metamorph software, Adobe Photoshop and Image J.

\section{Drug treatments}

\section{i. SB431542 to inhibit TGF- $\beta$ signaling}

Drug treatments were carried out in 24-well plates with up to 12 larvae per well in 1.5 $\mathrm{mL} 25 \mu \mathrm{M}$ SB431542 in $0.5 \%$ DMSO in egg water, or in $1.5 \mathrm{~mL} 0.5 \%$ DMSO in egg water in dark conditions. Larvae were treated beginning at $6 \mathrm{dpf}$ and placed in an incubator at $28.5^{\circ} \mathrm{C}$ for approximately 24 hours. Fresh SB431542 or DMSO solutions were replenished at $7 \mathrm{dpf}$, larvae were placed back in the $28.5{ }^{\circ} \mathrm{C}$ incubator in dark conditions for 24 hours and then fixed in AB Fix (4\% PFA, 0.1\% Triton-X, 1X PBS) at 8 dpf for 3 hours at $23{ }^{\circ} \mathrm{C}$ or overnight at $4{ }^{\circ} \mathrm{C}$. To validate SB431542 was working, onecell stage embryos were treated with either 100 or $200 \mu \mathrm{M}$ SB431542 in $0.5 \%$ DMSO in egg water or $0.5 \%$ DMSO in egg water until approximately 24 hours post fertilization. Embryos were assayed for gross developmental changes as previously described (Sun et al., 2006).

\section{ii. Clemastine fumarate}

Clemastine fumarate (Walgreen's Wal-Hist Clemastine Fumarate Tablets, USP $1.34 \mathrm{mg}$ Antihistamine) was dissolved in $1 \mathrm{~mL}$ of DMSO to create a stock concentration of $2 \mathrm{mM}$ clemastine. For injection experiments, the $2 \mathrm{mM}$ stock solution was diluted to either an 8 
$\mu \mathrm{M}$ working stock in $1 \mathrm{X}$ PBS or $4 \mu \mathrm{M}$ working stock in $1 \mathrm{X}$ PBS. Lysolecithin injection cocktails contained either $500 \mathrm{nM}$ clemastine $(5.25 \mu \mathrm{l}$ 1\% lysolecithin $+0.75 \mu \mathrm{l} 8 \mu \mathrm{M}$ clemastine) or $1 \mu \mathrm{M}$ clemastine $(5.25 \mu \mathrm{l} 1 \%$ lysolecithin $+0.75 \mu \mathrm{l} 4 \mu \mathrm{M}$ clemastine $)$.

\section{Individual oligodendrocyte labeling}

To mosaically label oligodendrocytes, fertilized eggs were injected with $12.5 \mathrm{ng} / \mu \mathrm{l}$ of a DNA plasmid encoding for mbp:mCherry-CAAX (Mensch et al., 2015) (courtesy of Dr. Lyons) and $25 \mathrm{ng} / \mu \mathrm{l}$ transposase RNA at the 1-cell stage.

\section{Spinal cord injections}

Lysolecithin (1-Palmityl-Sn-glycero-3-phosphocholine, Sigma L5254-25mg) was dissolved in saline at a $1 \%$ stock concentration and stored at $-20^{\circ} \mathrm{C}$. Thin wall glass microcapillaries (World Precision Instruments, Inc., Glass Thin Wall with Filament 1.0 mm OD, 4 in Length, Item No. TW100F-4) were pulled for microinjections using a micropipette puller (Sutter Instrument Co., Flaming/Brown Micropipette Puller, Model P97) and the capillary tip was opened using forceps (Student Dumont \#5 Inox Forceps, Fine Science Tools, Item \#91150-20). For experimental injections, lysolecithin was diluted to $0.875 \%$ concentration in saline and loaded into the glass microcapillary using a Microloader ${ }^{\mathrm{TM}}$ tip (Eppendorf Catalog No. 5242956003). For control injections, water or saline was loaded into the glass microcapillary using a Microloader ${ }^{\mathrm{TM}}$ tip. Larvae $4-6$ dpf were anesthetized using 3-aminobenzoic acid ester (Tricaine) and mounted laterally on to $2 \%$ agar pads solidified onto a borosilicate glass coverslip $(22 \mathrm{~mm} \times 60 \mathrm{~mm}$; Fisherbrand Cover Glasses: Rectangles, Catalog No. 12-545-J). The coverslip with 
mounted larvae was placed on to a Zeiss Axio Observer Z1 microscope stage equipped with an ASI MPPI-3 pressure injection rig and mechanical micromanipulator. The micropipette needle, containing either the control or lysolecithin solution, was inserted into the larvae, starting from the dorsal spinal cord and moving ventrally within the spinal cord, at approximately somite 16 - somite 18 . Solutions were dispersed passively into the spinal cord at a pressure range below $16 \mathrm{psi}$, until the dispersal region spanned the width of two to four somites. Following injections, the glass coverslip containing the mounted larvae was removed from the microscope stage and placed into a petri dish (Falcon Sterile Petri Dish, 100 x 15 mm style, REF 351029) with egg water. The coverslip and larvae were completely submersed in egg water until the agar pad was saturated, resulting in the larvae floating off the agar pad. A pipette pump (Bel-Art, Scienceware Pipette Pump $10 \mathrm{~mL}$ Pipettor, Cat. No. 378980000) with a $2 \mathrm{~mL}$ glass Pasteur pipet tip (VWR Pasteur Pipet Disposable BBD Borosilicate Glass, 5 3/4" size, Cat. No. 53283-916) was used to transfer the larvae into a separate petri dish with egg water to allow the larvae to recover.

\section{Data quantification and statistical analysis}

All graphically presented data represent the mean of the analyzed data. Statistical analyses and graphing were performed with GraphPad Prism software. The level of significance was determined by using an unpaired $t$ test or a Chi-Square test using a confidence interval of $95 \%$. 


\title{
Chapter 3
}

\section{Transmission electron microscopy of zebrafish spinal motor nerve roots}

\author{
Angela D. Morris, Alev Erisir, Stacey J. Criswell and Sarah Kucenas
}

Submitted for publication as a techniques article.

\begin{abstract}
Spinal motor nerves are essential for relaying information between the central and peripheral nervous systems. Perturbations to cell types that compose these nerves may impair rapid and efficient transmission of action potentials and alter nerve function. Identifying ultrastructural changes resulting from defects to these cellular components via transmission electron microscopy (TEM) can provide valuable insight into nerve function and disease. However, efficiently locating spinal motor nerves in adult zebrafish for TEM is challenging and time consuming. Because of this, we developed a protocol that allows us to quickly and precisely locate spinal motor nerve roots in adult zebrafish for TEM processing. Following fixation, a transverse slab of adult zebrafish dissected from the trunk region was mounted in embedding media, sectioned and secondary fixation with osmium tetraoxide was performed. Transverse sections containing motor nerves were selected for TEM ultrathin sectioning and imaging. We developed an efficient protocol for locating spinal motor nerves in adult zebrafish to allow for ultrastructural characterization. Although our work focuses on spinal motor nerves, this protocol may be useful for efficiently identifying other discrete, repeated structures within the developing and mature nervous system that are difficult to find via traditional, whole organism TEM processing.
\end{abstract}




\section{Introduction}

Transmission electron microscopy (TEM) is a powerful technique for investigating tissue ultrastructure, providing a much higher resolution than light microscopy that allows for visualization of cellular structures at a nanometer resolution (Lyons et al., 2008; Winey et al., 2014). Previously, TEM has been used by the zebrafish neuroscience community for investigating myelin ultrastructure within the central nervous system (CNS) (Czopka \& Lyons, 2011; Lyons et al., 2008) and along the peripheral nervous system (PNS) lateral line nerve (Monk et al., 2009). Both the spinal cord and lateral line system are relatively easy to locate for electron microscopy processing. The spinal cord extends the length of the trunk and the superficial lateral line system is also a continuous structure found along the length of the zebrafish body, and is easily dissected or harvested for EM processing. In contrast, spinal motor nerve roots lie deep within the zebrafish somatic muscle and the axons that make up these structures exit into the periphery via motor exit point (MEP) transition zones (TZ), which are discrete structures that are located in the center of each body segment in an iterated fashion along the trunk. Because of this, efficiently and precisely locating spinal motor roots in traditionally processed TEM sections is challenging. Although zebrafish embryos and larvae are optically transparent and transgenes or antibody labeling can be used to label motor axons for easy identification, adult zebrafish lack optical transparency (Figure 3-1A), making it difficult to efficiently locate spinal motor nerves when processing tissue for EM.

Because spinal motor nerves are essential for relaying information between the CNS and peripheral targets, functional changes in these conduits can lead to devastating 
consequences in target maintenance and function. Investigating the ultrastructural changes that occur to spinal motor nerves after genetic manipulation or injury can provide a better understanding of the role of specific genes or cells in forming or maintaining a functional nerve. Because locating spinal motor nerve roots in adult zebrafish is difficult and time intensive, we have taken advantage of several methods to create a protocol that allows us to efficiently and rapidly locate spinal motor nerve roots within the adult zebrafish trunk (Figure 3-1A). This allows us to directly and precisely investigate the ultrastructure of these essential nervous system components in two different planes of section: a cross-sectional view that is transverse (Figure 3-1B) or perpendicular to the spinal motor nerve axis and a coronal/frontal orientation (Figure 31C) that is longitudinal to the axis of spinal motor nerves. In this protocol, we focused on the adult zebrafish spinal motor nerve root. However, this protocol can be adapted for use in identifying other discrete structures within the nervous system, as well as during other developmental stages, allowing for a more rapid and precise processing and sectioning of structures for TEM.

\section{Results \& Discussion}

\section{Fixation of adult zebrafish samples}

We have combined several techniques to develop a protocol for efficiently identifying and evaluating the ultrastructure of spinal motor nerve roots in adult zebrafish. After euthanizing adult zebrafish by immersion in $0.4 \%$ Tricaine, a small piece of fin tissue was collected for genotyping and trunk samples of at least $0.5 \mathrm{~cm}$ were dissected using a 


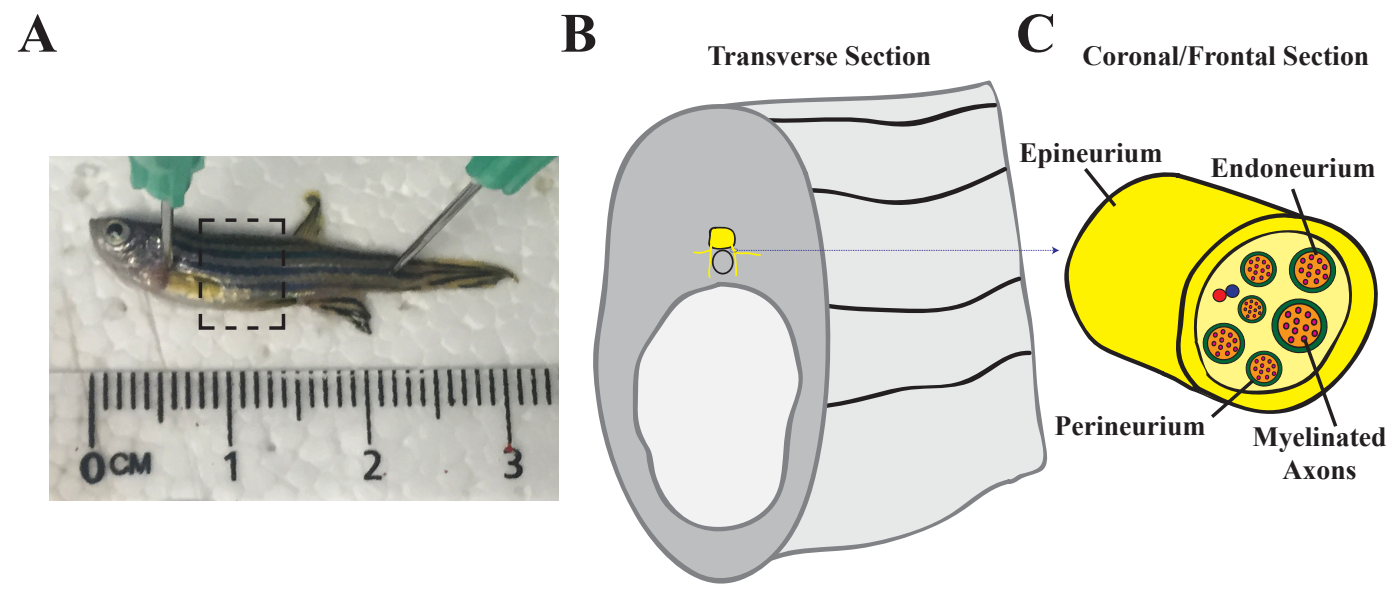

Figure 3-1. Spinal motor nerves in adult zebrafish. (A) Image of an adult zebrafish with anterior to the left and dorsal to the top. A dashed box identifies the trunk region of interest. (B) Cartoon schematic of a transverse section of the trunk region, identifying the nervous system in yellow. The purple arrow denotes the spinal motor nerve root, the region of interest for coronal/frontal sections. (C) Cartoon schematic of a coronal/frontal cross-section of a spinal motor nerve. Myelinated axons (magenta) are surrounded by the endoneurium (orange). Several myelinated axons are ensheathed by the perineurium (green) to form a nerve fascicle, and several nerve fascicles are brought together into a nerve bundle by the epineurium (yellow). 
razor blade and placed in a polypropylene tube containing Karnovsky's fixative (4\% paraformaldehyde and 2\% gluteraldehyde in $0.1 \mathrm{M}$ sodium cacodylate) (Lyons et al., 2008). The polypropylene tube was placed in a beaker containing ice water to keep the sample at approximately $15^{\circ} \mathrm{C}$ to prevent overheating during fixation, and placed in a microwave with adjustable power settings. A modified microwave fixation protocol (Chan et al., 1990; Czopka \& Lyons, 2011; Lyons et al., 2008; Lyons et al., 2009) was used to accelerate fixation. After loosening the lid on the polypropylene tube, the following microwave fixation steps were performed: power level 2 (250 watts) for 2 minutes, off for 2 minutes, on for 2 minutes; power level 1 (125W) for 2 minutes, off for 2 minutes, on for 2 minutes. Following microwave fixation, samples were removed from the polypropylene tube using forceps, placed in fresh Karnovsky's fixative in a 24 -well plate and were left shaking at room temperature for at least 2 hours. The Karnovsky's fixative was removed from the wells and replaced with $2.5 \%$ gluteraldehyde in $0.1 \mathrm{M}$ sodium cacodylate. Samples were then stored at $4^{\circ} \mathrm{C}$ overnight.

\section{Sample preparation for microtome sectioning}

Following genotyping, trunk samples were removed from $2.5 \%$ gluteraldehyde in $0.1 \mathrm{M}$ sodium cacodylate and mounted in a Peel-A-Way ${ }^{\circledR}$ disposable embedding mold (T-8) containing $1.75 \%$ gluteraldehyde in a gelatin/albumin embedding media platform (Figure 3-2A). Trunk samples were mounted posterior side facing the embedding media platform and $1.75 \%$ gluteraldehyde in gelatin/albumin embedding media was added to the embedding mold to encase the entire sample (Figure 3-2A). After the embedding media solidified at room temperature, the sample was removed from the embedding mold, 
trimmed down in size to a cube using a razor blade and mounted on the vibratome platform, anterior side down (Figure 3-2B). Using a Leica VT1000S vibrating blade microtome, frequency setting at 7 and speed setting at $1,200 \mu \mathrm{m}$ transverse sections (Figure 3-2C) were cut and placed in a well plate containing 0.1M sodium cacodylate until initial TEM processing occurred. Alternatively, at this stage, the samples can be processed for immunohistochemistry by using an antibody to label for motor axons or Black Gold II to stain for myelin. Samples may be removed from the gelatin/albumin embedding media using forceps and a soft-bristled paintbrush, or kept within the embedding media, for the IHC protocols described below.

For antibody labeling, we adapted an immunohistochemistry on floating sections protocol to visualize motor axons. We replaced the $0.1 \mathrm{M}$ sodium cacodylate with a blocking solution ( $1 \%$ BSA / $2 \%$ goat serum / $0.05 \%$ Triton-X in $1 \mathrm{X}$ PBS) and incubated the samples in block at room temperature for 40 minutes, not shaking. The block solution was replaced with an anti-acetylated tubulin antibody (mouse, 1:5000; Sigma, T7451) in block solution without Triton-X, and incubated at $4^{\circ} \mathrm{C}$ overnight. Samples were washed three times in $1 \mathrm{X}$ PBS at room temperature and placed in a goat anti-mouse secondary antibody in block solution that lacked Triton-X. Samples were incubated in the secondary antibody solution and covered for 2 hours at room temperature. Samples were then washed with $1 \mathrm{X}$ PBS, two times, 15 minutes per wash and screened for tubulin labeling using a Zeiss AxioZoom.V16 fluorescence stereo zoom microscope. Spinal motor root axons were easily identified based on tubulin labeling (Figure 3-3A). 
A

B
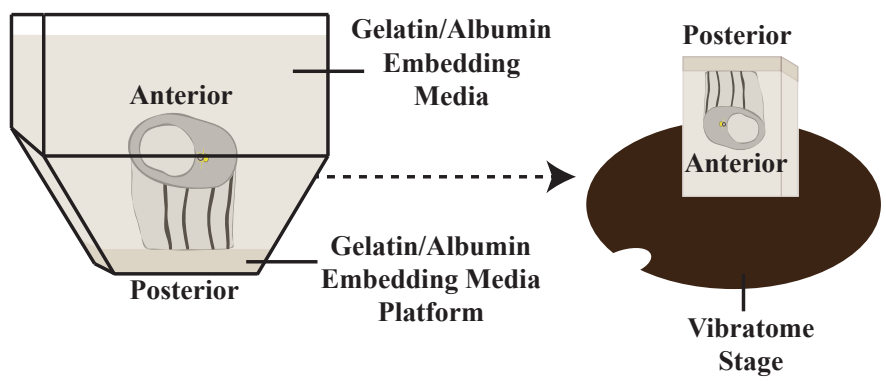

C

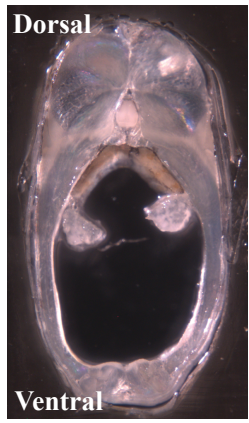

Figure 3-2. Vibrating blade microtome transverse sections of adult zebrafish trunk samples. (A) Cartoon schematic portraying the dissected trunk region embedded in gelatin / albumin. (B) Following embedding media solidification, the sample is removed from the mold and trimmed down using a razor blade. The sample is mounted on the vibratome platform for sectioning. (C) $200 \mu \mathrm{m}$ transverse section of an adult zebrafish trunk region with dorsal to the top. 
Alternatively, the myelin sheath associated with spinal motor axons can be visualized using a Black Gold II myelin staining kit (Histo-Chem Inc., Product IBGII). Black Gold II specifically labels myelin (Schmued et al., 2008) and has previously been used to label myelin in the zebrafish brain (Larson et al., 2010). Black Gold II labeling of individual myelinated axons appears black whereas large myelinated tracts, like the spinal motor nerve root, appear deep red to brown in color. After collection via microtome sectioning, the $0.1 \mathrm{M}$ sodium cacodylate was replaced with Black Gold II stain and samples were incubated at $60^{\circ} \mathrm{C}$ for approximately 1 hour. Samples were rinsed in $1 \mathrm{X}$ PBS for 2 minutes at room temperature and in $1 \%$ sodium thiosulfate for 3 minutes at room temperature. Samples were stored in 1X PBS until imaged using a Zeiss AxioZoom.V16 stereo zoom microscope and the spinal motor nerve roots were easily identified based on the Black Gold II labeling (Figure 3-3B).

\section{Initial TEM processing}

Transverse sections selected for ultrastructural analysis based on the presence of spinal motor nerves via immunohistochemistry or Black Gold II labeling were processed for electron microscopy using standard protocols (Chan et al., 1990; Corson et al., 2009; Erisir \& Dreusicke, 2005; Irwin et al., 2016). Sections were washed in 0.1M sodium cacodylate, 2 times, 3 minutes per wash, at room temperature. Samples were gently removed from the gelatin/albumin embedding media using forceps and a soft bristled paintbrush. Samples were transferred to a glass scintillation vial using a paintbrush. For the secondary fixation, the samples were completely immersed in $1 \%$ osmium tetraoxide $(\mathrm{OsO} 4)$ in $0.1 \mathrm{M}$ sodium cacodylate and incubated at room temperature for 1 hour. 
Following incubation, $1 \% \mathrm{OsO} 4$ in $0.1 \mathrm{M}$ sodium cacodylate was extracted from the glass scintillation vial and samples were rinsed in $0.1 \mathrm{M}$ sodium cacodylate, 3 times, 3 minutes per wash.

\section{Sample dehydration}

All steps were done at room temperature. Samples were dehydrated sequentially: 50\% ethanol for 3 minutes, $4 \%$ uranyl acetate in $70 \%$ ethanol for at least 1 hour to overnight, $70 \%$ ethanol for 1 minute, $90 \%$ ethanol for 5 minutes, and $100 \%$ ethanol twice at 5 minutes per exposure. Samples were then rinsed in acetone 3 times, 2 minutes per rinse. Following removal of the final acetone rinse, samples were stored in a 1:1 EPON:acetone mixture overnight. Following removal of the 1:1 EPON:acetone mixture, EPON was placed in the glass scintillation vial and samples were incubated overnight. Samples were transferred from the EPON in the glass scintillation vial to a piece of ACLAR ${ }^{\circledR}$ embedding film to screen for spinal motor nerves. Since osmium reacts with membrane lipids, osmium labeling of the myelin sheath can be used to identify spinal motor nerves (Figure 3-3C). Sections containing spinal motor nerves were processed for ultrathin sectioning in one of two orientations: coronal/frontal (i.e., longitudinal to motor nerve axis) or transverse (i.e., perpendicular to motor nerve axis).

\section{TEM processing for ultrathin coronal/frontal cross-sections}

A thin layer of EPON was added to the bottom of each well within a small flat embedding mold and prebaked at $65^{\circ} \mathrm{C}$ for approximately 6 hours. Samples that we identified as containing spinal motor nerves were transferred to those individual wells. 
$\mathbf{A}$

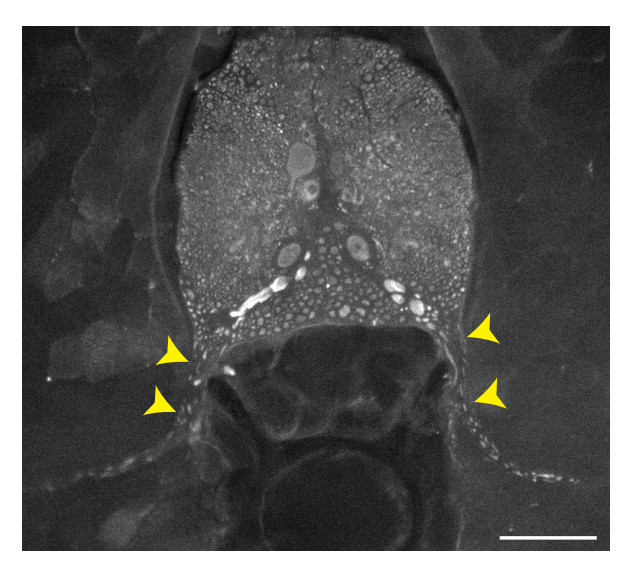

B

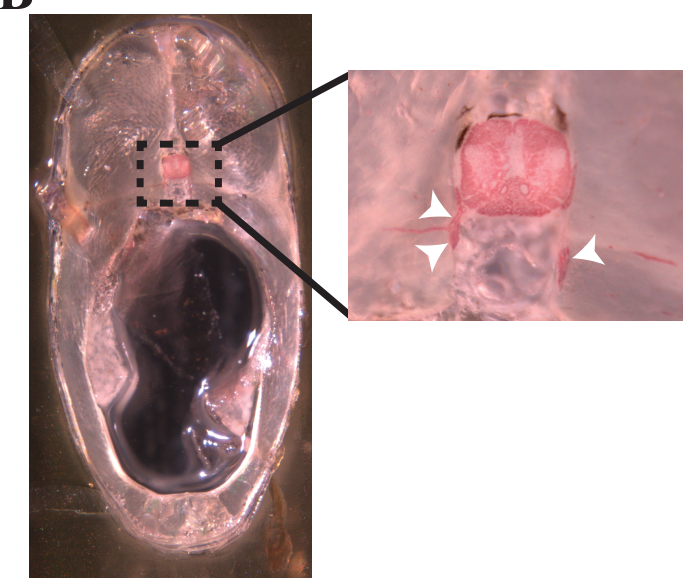

C

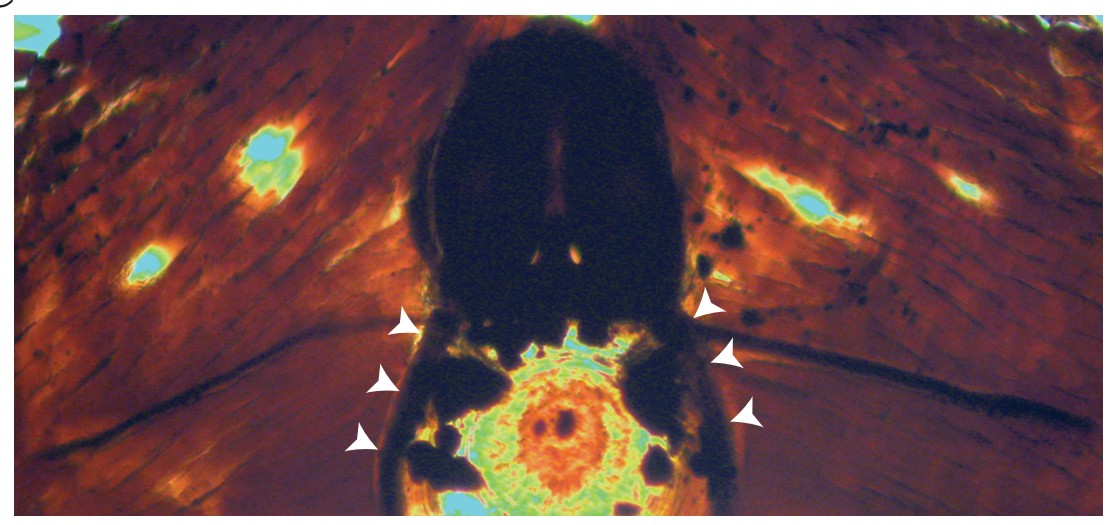

Figure 3-3. Locating spinal motor nerves in adult zebrafish transverse sections.

(A) Immunohistochemistry on floating sections using an antibody to tubulin. Yellow arrowheads identify the tubulin ${ }^{+}$peripheral motor axons. Scale bar, $100 \mu \mathrm{m}$. (B) Black gold II myelin stain to label the myelin sheath. The dotted box denotes the area enlarged in the right image. White arrowheads identify the myelin sheath associated with peripheral motor axons. (C) Osmium labeling identifies the myelin sheath associated with peripheral motor axons (white arrowheads). 
The remainder of each well was filled with EPON and placed in a $65^{\circ} \mathrm{C}$ oven overnight to polymerize the EPON (Figure 3-4A). Following polymerization, a clean razor blade was used to cut the dorsal region of the sample (Figure 3-4B) so that ultrathin sectioning of the motor nerve would begin approximately adjacent to the ventral spinal cord, just dorsal of the notochord. To confirm the region selected for ultrathin sectioning was in the periphery and would capture the peripheral motor nerve roots, approximately $0.5 \mu \mathrm{m}$ thick coronal/frontal cross-sections were collected on a glass slide and stained with toluidine blue (Figure 3-4C). After trimming the block to contain only the desired region, a Leica EM UC7 ultramicrotome was used to cut the ultrathin sections at $70 \mathrm{~nm}$. Ultrathin sections were collected on 200 mesh copper grids and counterstained using uranyl acetate and lead citrate. The grids were coated with a thin layer of carbon using a Bal-Tec Med020 coating system to minimize conductance and improve stability in the TEM. Samples were stored at room temperature until imaging.

\section{TEM processing for ultrathin transverse sections}

Samples containing spinal motor nerves to be used for the transverse section orientation were transferred to an ACLAR ${ }^{\circledR}$ embedding film that had been cleaned with ethanol. EPON was dispersed around each sample using a metal micro-spatula and sections were flat embedded by placing an ACLAR ${ }^{\circledR}$ embedding film on top of the samples (Figure 34D). Bubbles were gently removed from around the samples. The flat embedded samples were placed in a $60^{\circ} \mathrm{C}$ oven overnight to polymerize the EPON. Samples were removed from the ACLAR ${ }^{\circledR}$ embedding sheets and re-embedded in BEEM ${ }^{\circledR}$ embedding capsules. The embedding capsules were filled with EPON and placed in a $60^{\circ} \mathrm{C}$ oven overnight to 
A

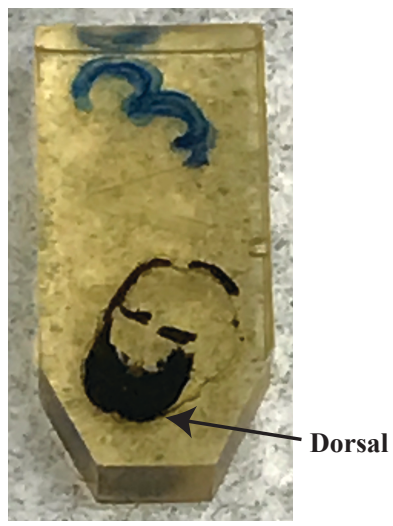

D

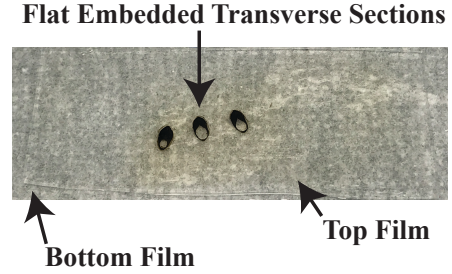

B
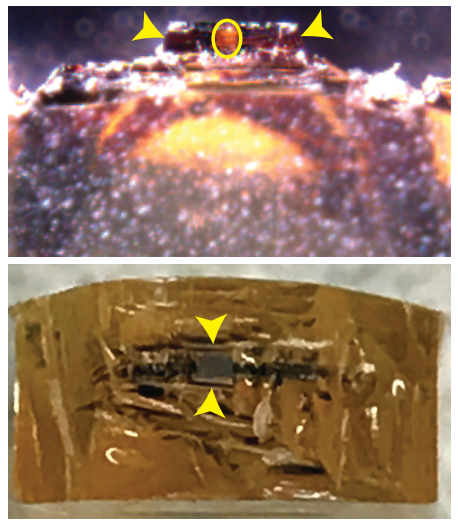

$\mathbf{E}$

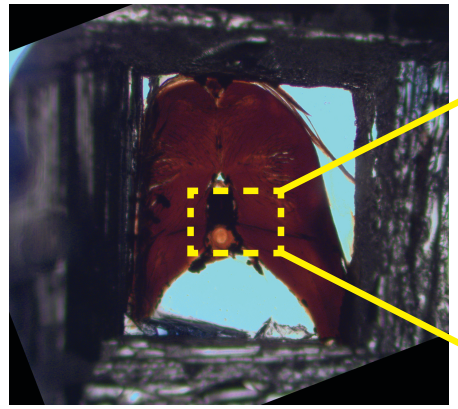

C
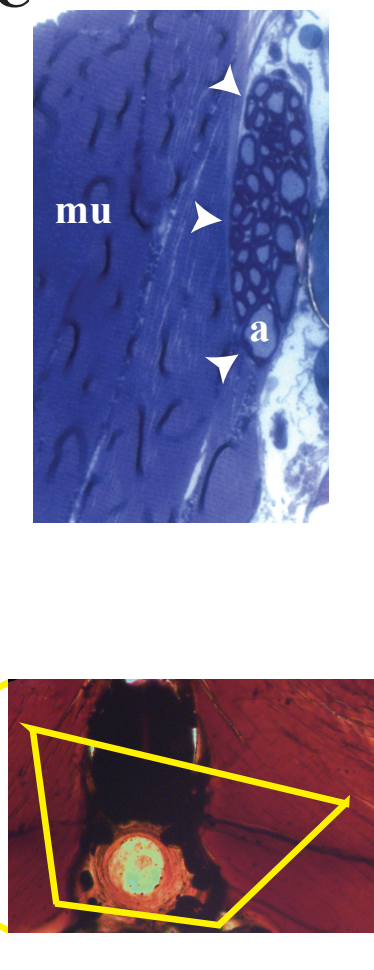

Figure 3-4. Embedding transverse sections for ultrathin sectioning in coronal / frontal and transverse orientations. (A) Transverse sections embedded within a flat mold, dorsal side oriented towards tip of mold to allow for coronal/frontal sections of the motor nerve. (B) For coronal/frontal sections, a razor blade is used to remove the dorsal region of the sample. A trapezoid is created containing the region of interest (ROI) for ultrathin sectioning (Top: lateral view; Bottom: top view). Yellow circle denotes notochord. Yellow arrowheads identify trapezoid. (C) To confirm the ROI is capturing a coronal/frontal cross-section of the motor nerves, $0.5 \mu \mathrm{m}$ thick sections of a large area (several $\mathrm{mm}$ ) are collected on a glass slide and stained with toluidine blue. Myelinated axons (a) are seen within the peripheral nerve (white arrowheads). Muscle $(\mathrm{mu})$ is adjacent to the peripheral nerve. (D) For transverse sections, samples are flat embedded in EPON between two ACLAR ${ }^{\circledR}$ films. (E) Flat embedded samples are excised from the film and re-embedded in a capsule. Polymerized EPON is removed to expose the sample (left) and a trapezoid ROI is identified (right). A trapezoid is created based on the identified ROI and ultrathin sectioning in a transverse orientation is performed. 
polymerize the EPON. Block-embedded sections were examined and imaged on a light microscope to identify the region of interest, which should be no larger than a trapezoid with $1 \mathrm{~mm} \times 2 \mathrm{~mm}$ dimensions. Using a clean razor blade, the block was trimmed down to the region of interest, which contained the midline and the osmium accentuated motor nerve (Figure 3-4E). A Leica Ultracut UCT Ultramicrotome was used for ultrathin sections of $70 \mathrm{~nm}$. Ultrathin sections were transferred to copper grids and stored at room temperature until TEM imaging occurred.

\section{TEM imaging of spinal motor nerve roots}

Ultrathin sections may be visualized using a transmission electron microscope or a variable pressure scanning electron microscope with a STEM detector. For our study, coronal/frontal cross sections of adult motor nerves were visualized using a Zeiss Sigma HD variable pressure scanning electron microscope with a STEM detector (Figure 3-5A) and transverse sections were visualized using a JEOL 1010 Transmission electron microscope (Figure 3-5B).

In conclusion, the protocol we describe allows for efficient and rapid localization of spinal motor nerve roots in adult zebrafish for subsequent EM characterization. Although our focus was on adult tissue, this protocol may be useful for imaging other discrete structures in the zebrafish nervous system as well as at different developmental stages. 
A

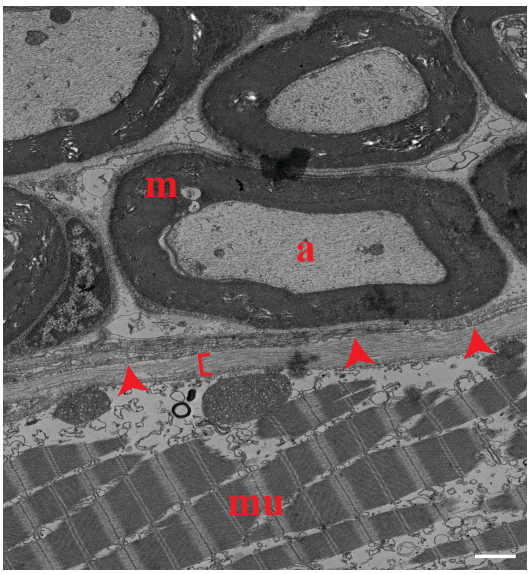

B

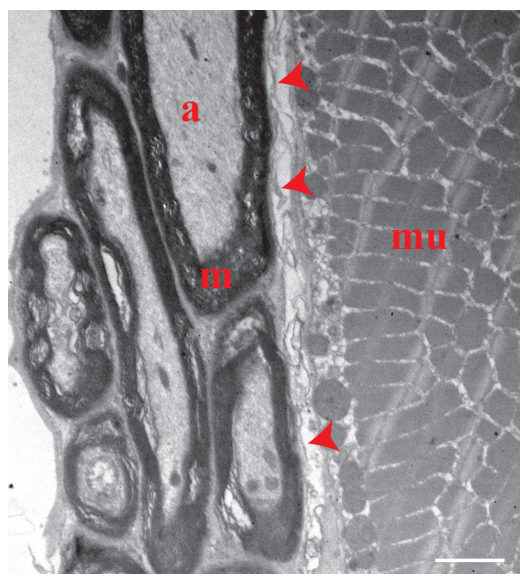

Figure 3-5. Ultrastructural evaluation of adult zebrafish motor nerves. (A) Coronal/frontal view of an adult zebrafish motor nerve. (B) Transverse view of an adult zebrafish motor nerve. a: axon; m: myelin; mu: muscle; arrowheads: perineurium; bracket: epineurium. Scale bars, $1 \mu \mathrm{m}$. 
Acknowledgements: We would like to thank members of the Kucenas and Erisir laboratories for valuable discussions and Lori Tocke for zebrafish care. We would also like to thank Bonnie Sheppard from the Erisir lab and Dr. Yalin Wang from the UVA Advanced Microscopy Facility for their electron microscopy assistance, and Anthony Fernandez-Castaneda and Alban Gaultier for sharing their immunohistochemistry expertise and solutions. This work was supported by the National Institutes of Health (NIH): NS072212 (SK), the March of Dimes: 5-FY11-90 (SK) and the Hartwell Foundation (SK). 


\title{
Chapter 4
}

\section{Perineurial glial plasticity and the role of TGF- $\beta$ in the development of the blood-nerve barrier}

Angela D. Morris, Gwendolyn M. Lewis and Sarah Kucenas

Accepted for publication in The Journal of Neuroscience, April 7, 2017

\begin{abstract}
Precisely orchestrated interactions between spinal motor axons and their ensheathing glia are vital for forming and maintaining functional spinal motor nerves. Following perturbations to peripheral myelinating glial cells, centrally-derived oligodendrocyte progenitor cells (OPCs) ectopically exit the spinal cord and myelinate peripheral nerves in myelin with central nervous system (CNS) characteristics. However, whether remaining peripheral ensheathing glia, such as perineurial glia, properly encase the motor nerve despite this change in glial cell and myelin composition, remains unknown. Using zebrafish mutants in which OPCs migrate out of the spinal cord and myelinate peripheral motor axons, we assayed perineurial glial development, maturation and response to injury. Surprisingly, in the presence of OPCs, perineurial glia exited the CNS normally. However, aspects of their development, response to injury and function were altered when compared to wildtype larvae. In an effort to better understand the plasticity of perineurial glia in response to myelin perturbations, we identified transforming growth factor beta 1 (TGF- $\beta 1)$ as a partial mediator of perineurial glial development. Taken together, these results demonstrate the incredible plasticity of perineurial glia in the presence of myelin perturbations.
\end{abstract}




\section{Significance statement}

Peripheral neuropathies can result from damage or dysregulation of the insulating myelin sheath surrounding spinal motor axons, causing pain, inefficient nerve conduction and the ectopic migration of oligodendrocyte progenitor cells (OPCs), the resident myelinating glial cell of the central nervous system (CNS), into the periphery. How perineurial glia, the ensheathing cells that form the protective blood-nerve barrier (BNB), are impacted by this myelin composition change is unknown. Here, we report that certain aspects of perineurial glial development and injury responses are mostly unaffected in the presence of ectopic OPCs. However, perineurial glial function is disrupted along nerves containing centrally-derived myelin, demonstrating that although perineurial glial cells display plasticity despite myelin perturbations, the blood-nerve barrier is compromised in the presence of ectopic OPCs.

\section{Introduction}

Formation of an efficient and functional peripheral nervous system (PNS) requires precise and coordinated interactions between several distinct cell populations (Akert et al., 1976; Appel et al., 1995; Bourne, 1968; Corfas et al., 2004; Gamble \& Breathnach, 1965; Kucenas et al., 2008b; Shanthaveerappa \& Bourne, 1966). Spinal motor nerves, the conduits by which information is conveyed from the central nervous system (CNS) to peripheral targets such as muscles, glands and organs, are composed of motor axons and their associated ensheathing glia (Appel et al., 1995; Corfas et al., 2004; Kucenas et al., 2008b). Given the complexity required to form and maintain a functional motor nerve, it is not surprising that aberrations may arise, resulting in disease states like peripheral 
neuropathies, including Charcot-Marie-Tooth disease and congenital peripheral amyelinopathy. Some forms of neuropathies attack the myelin sheath (Hughes, 2002). However, how and if remaining ensheathing glial cell populations, such as perineurial glia, compensate and maintain a functional nerve despite myelin perturbations, is not well understood.

During development, CNS-derived motor exit point (MEP) glia and neural crest-derived Schwann cells myelinate spinal motor axons (Emery, 2010; Jessen \& Mirsky, 2005; Smith et al., 2014). CNS-derived perineurial glia exit the spinal cord and bundle several myelinated axons into a nerve fascicle, eventually differentiating into the mature perineurium, a component of the blood-nerve barrier (Akert et al., 1976; Bourne, 1968; Clark et al., 2014; Jessen \& Mirsky, 2005; Kristensson \& Olsson, 1971; Kucenas et al., 2008b; Rowitch, 2004; Smith et al., 2014). In both fish and mouse, perineurial glia are required for motor axon pathfinding from the spinal cord, restricting ectopic migration of motor neuron cell bodies into the periphery and are necessary for Schwann cell development and peripheral myelination (Binari et al., 2013; Clark et al., 2014; Kucenas et al., 2008b). Additionally, we recently discovered perineurial glia are essential mediators of nerve regeneration by phagocytizing debris and forming glial bridges across injury sites (Lewis \& Kucenas, 2014). Although we have begun to reveal the importance of perineurial glia in development, we still lack an understanding of how they respond in disease states, like peripheral myelinopathies. 
Following peripheral myelin perturbations in zebrafish, mice and humans, oligodendrocytes are found ectopically along spinal motor root axons (Coulpier et al., 2010; Kucenas et al., 2009; Lewis \& Kucenas, 2014; Smith et al., 2014; Weinberg et al., 1975). However, nothing is known about how perineurial glia are affected by this change in glial composition. Here, we investigate whether perineurial glial development, maturation, function and response to injury are altered in the presence of ectopicallylocated OPCs and central myelin. By characterizing perineurial glial phenotypes in a zebrafish mutant with peripheral OPCs $\left(e r b b 3 b^{-/}\right)$(Smith et al., 2014), we show that aspects of perineurial glial development and injury response behaviors are comparable to wildtype controls, and that TGF- $\beta 1$ plays a role in perineurium maturation. Our results demonstrate the adaptability of perineurial glial cells despite the presence of ectopic

OPCs. However, the functionality of the perineurium fails, further supporting the importance of Schwann cell-perineurial glial interactions during spinal motor nerve development and maintenance (Parmantier et al., 1999; Sharghi-Namini et al., 2006).

\section{Results}

$e r b b 3 b^{-/}$spinal motor nerves as a model to study perineurial glial plasticity in response to myelin perturbations

The five main cellular components that comprise vertebrate spinal motor nerves (Figure 4-1A) are motor axons, peripheral myelinating glial cells, such as motor exit point (MEP) glia and Schwann cells, endoneurial components, perineurial glia and epineurial cells (Figure 4-1B) (Gamble, 1966; Gamble \& Breathnach, 1965; Gamble \& Eames, 1964; Jessen \& Mirsky, 1999; Maurel \& Salzer, 2000; Plessis et al., 1996; Smith et al., 2014; 


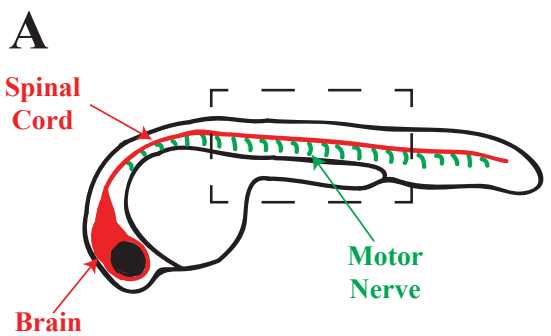

B
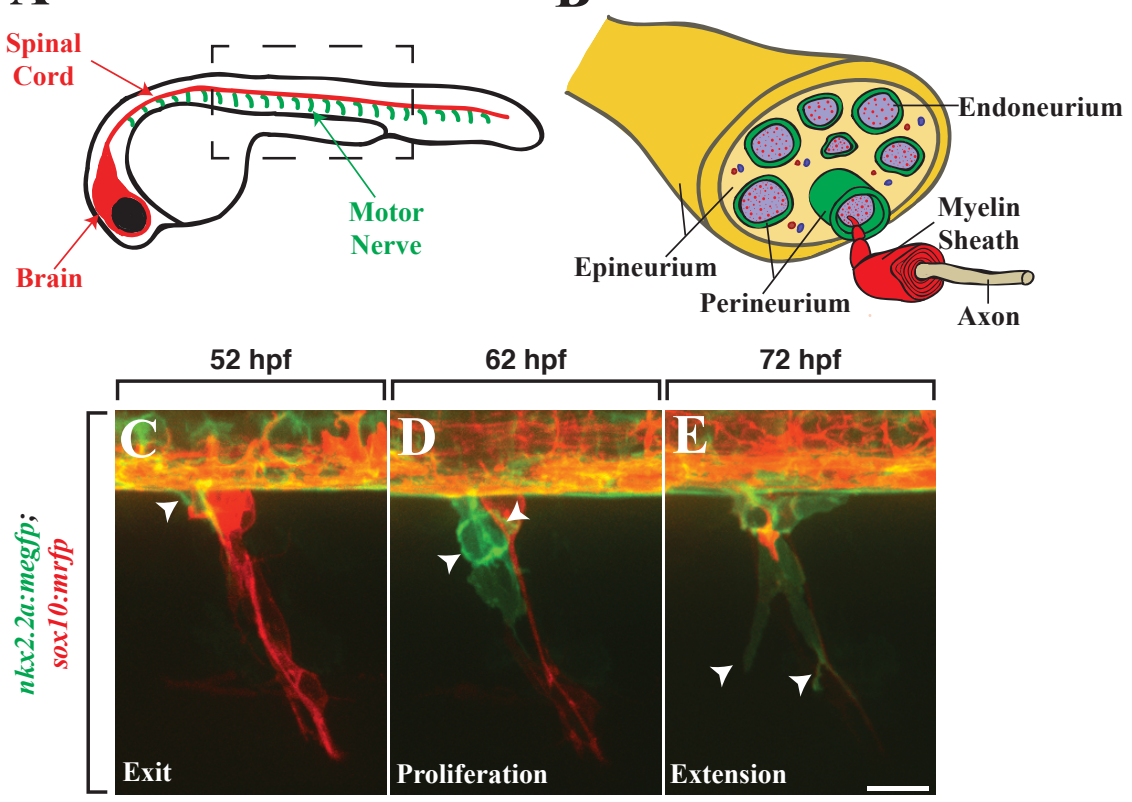

Figure 4-1. Perineurial glial development in zebrafish. (A) Cartoon of a zebrafish embryo identifying the CNS, which consists of the brain and spinal cord (red), and the peripheral spinal motor nerves (green). (B) Diagram representing a cross sectional view of an adult peripheral motor nerve. Axons are wrapped by peripheral myelinating glial cells (myelin sheath, red) and are surrounded by the endoneurium (purple). Several myelinated axons are bundled by the perineurium (green) to form a fascicle. The epineurium (yellow) then encases several fascicles to form a nerve. $(\mathrm{C}-\mathrm{E})$ All images are lateral views of the spinal cord with dorsal to the top and anterior to the left. In wildtype $n k x 2.2 a$ :megfp;sox10:mrfp zebrafish larvae, $n k x 2.2 a^{+}$perineurial glia (arrowheads) exit the CNS by approximately $52 \mathrm{hpf}(\mathrm{C})$, proliferate (D) and extend (E) until full ensheathment of the motor nerve is achieved. Scale bar, $25 \mu \mathrm{m}$. 
Thomas, 1963). In both mouse and zebrafish, perineurial glia originate from precursors in the p3 domain (floor plate) of the spinal cord and exit into the periphery via MEP transition zones (TZ) (Clark et al., 2014; Kucenas et al., 2008b). In zebrafish, perineurial glial migration is relatively stereotypic, with perineurial cells beginning to exit from the spinal cord at approximately 48 hours post fertilization (hpf), proliferating and then extending and retracting along the motor nerve until full ensheathment of the motor nerve to the neuromuscular junction (NMJ) is achieved, beginning at approximately 4 days post fertilization (dpf) (Figure 4-1C - 4-1E) (Kucenas et al., 2008b). We can visualize these migratory and extension behaviors using in vivo, time-lapse imaging of nkx2.2a:megfp;sox10:mrfp embryos, where regulatory sequences of $n k x 2.2 a$ and sox10 drive expression of membrane-tethered EGFP and RFP in perineurial glia and peripheral myelinating glia, respectively (data not shown).

After ensheathment, perineurial glia ultimately differentiate into the mature perineurium, a component of the blood-nerve barrier, providing structural and permeability support to the nerve (Akert et al., 1976). Tight junctions have previously been used to reliably and reproducibly detect the differentiated perineurium (Akert et al., 1976; Binari et al., 2013; Clark et al., 2014; Kristensson \& Olsson, 1971; Kucenas et al., 2008b; Lewis \& Kucenas, 2014), and zonula occludens-1 (ZO-1) has been used as a marker for detecting these structures (Binari et al., 2013; Clark et al., 2014; Kucenas et al., 2008b; Lewis \& Kucenas, 2014; Pummi et al., 2004). In zebrafish, ZO-1 labeling can be visualized at the MEP at $6 \mathrm{dpf}$, and as development proceeds, this labeling gradually extends distally along the nerve as perineurial glial cells continue to mature and become a component of 
the protective blood-nerve barrier (Binari et al., 2013; Kucenas et al., 2008b; Lewis \& Kucenas, 2014).

Previously, we demonstrated that perturbing Schwann cell development prevents (Kucenas et al., 2009) or delays (Kucenas et al., 2008b; Lewis \& Kucenas, 2014) perineurial glial migration from the spinal cord. Specifically, in mont blanc (mob ${ }^{\text {m610-/ }}$ ) (Barrallo-Gimeno et al., 2004) and mother superior $\left(\operatorname{mos}^{\mathrm{m} 188-/-}\right)$ double mutant embryos, which harbor mutations in the transcription factors tfap $2 a$ and foxd3, respectively, there is a failure of neural crest induction, and therefore, Schwann cells are absent along motor nerves (Arduini et al., 2009; Barrallo-Gimeno et al., 2004; Kucenas et al., 2009; Montero-Balaguer et al., 2006; Wang et al., 2011). In colourless $\left(c l s^{t w 11-/}\right)$ embryos and larvae, which harbor a mutation in the transcription factor sox10 (Dutton et al., 2001), neural crest-derived Schwann cells migrate to nascent spinal motor nerves, but die soon after associating with motor axons as Sox10 is required for Schwann cell survival and differentiation (Britsch, 2001; Kucenas et al., 2008b; Kuhlbrodt et al., 1998; Lewis \& Kucenas, 2014; Paratore et al., 2001). Time-lapse imaging of both mutants prior to $3 \mathrm{dpf}$ revealed that perineurial glia failed to exit the CNS (Kucenas et al., 2009). However, imaging $\mathrm{cls}^{-/-}$larvae at later time points in development revealed that perineurial glial migration was only delayed, not completely absent, along $\mathrm{cls}^{-/-}$motor nerves (Lewis \& Kucenas, 2014). Furthermore, in both $\mathrm{mob}^{-/-} ; \mathrm{mos}^{-/-}$and $\mathrm{cls}^{-/-}$larvae, the lack of all peripheral myelinating glia leads to the ectopic migration of OPCs into the periphery (Kucenas et al., 2009; Kucenas et al., 2008b). However, neither mutant is homozygous viable. Intriguingly, ectopically-located oligodendrocytes and centrally-derived myelin 
have previously been reported in the periphery of zebrafish, mice and humans (Coulpier et al., 2010; Kucenas et al., 2009; Lewis \& Kucenas, 2014; Smith et al., 2014; Weinberg et al., 1975). However, the impact that this abnormality has on remaining peripheral ensheathing glia, specifically perineurial glia, has not been investigated. To address this, we turned to another peripheral myelin mutant, $e r b b 3 b^{-/}$(Lyons et al., 2005), which has ectopic OPCs, centrally-derived myelin in the periphery (Smith et al., 2014), is homozygous viable and has normal swimming, feeding and mating behaviors (Honjo et al., 2011).

During development, erbb3, a receptor tyrosine kinase expressed by Schwann cells and their precursors, forms a heterodimer complex with erbb2 (Birchmeier, 2009; Brinkmann et al., 2008; Garratt et al., 2000b; Garratt et al., 2000a; Lyons et al., 2005; Newbern \& Birchmeier, 2010; Perlin et al., 2011). This erbb2-erbb3 complex then binds to the axonally-expressed growth factor Neuregulin I Type III (Nrg1), which promotes Schwann cell survival, proliferation and differentiation into myelinating glial cells (Birchmeier, 2009; Lyons et al., 2005; Newbern \& Birchmeier, 2010; Pereira et al., 2012; Perlin et al., 2011; Raphael et al., 2011). In erbb3b $b^{-/}$embryos and larvae, Schwann cell migration, proliferation, differentiation and myelination along the mechanosensory posterior lateral line (pLLn) is disrupted (Lyons et al., 2005; Riethmacher et al., 1997b; Torii et al., 2014) and OPCs ectopically exit the CNS, associate with and myelinate peripheral spinal motor nerve axons (Smith et al., 2014). Similarly, the loss of ErbB receptors in mice results in Schwann cell defects and hypomyelination of peripheral axons (Brinkmann et al., 2008; Riethmacher et al., 1997b). 
To validate that spinal motor nerves completely lack peripheral myelinating glial cells in $e r b b 3 b^{-/}$larvae, we performed in vivo imaging on $n k x .2 .2 a: m e g f p ; s o x 10: m r f p ; e r b b 3 b^{-/-}$ and wildtype embryos, where sox 10 regulatory sequences drive expression of membranetethered RFP in CNS and PNS myelinating glia. In wildtype larvae imaged at $55 \mathrm{hpf}$, we observed $\mathrm{RFP}^{+}$Schwann cells and MEP glia along wildtype spinal motor nerves (Figure 4-2A). In contrast, at $53 \mathrm{hpf}$ in $e r b b 3 b^{-/}$larvae, we observed spinal motor nerves that completely lacked $\mathrm{RFP}^{+}$peripheral glia (Figure 4-2B) (Smith et al., 2014). To confirm the presence of ectopically-located OPCs along mutant nerves, we performed in vivo, time-lapse imaging of $n k x 2.2 a: m e g f p ; o l i g 2: d s r e d ; e r b b 3 b^{-/}$larvae and observed $n k x 2.2 a^{+} /$olig $2^{+}$cells exit the CNS at MEP TZs beginning at approximately $59.5 \mathrm{hpf}$ (Figure 4-2C). This combination of transgenes allowed us to confidently call these cells OPCs because previous studies demonstrate that OPCs express both $n k x 2.2 a$ and olig2, and cells with this expression pattern are only ever observed within the CNS in wildtype larvae (Kucenas et al., 2008a). Once in the periphery, these ectopic OPCs migrated along motor axons until they ultimately associated with single axons and initiated ensheathment, as evidenced by the creation of thin, $n k x 2.2 a^{+}$rectangular tubes (data not shown), a phenotype consistent with glial ensheathment/myelination (Czopka et al., 2013; Kucenas et al., 2009; Nawaz et al., 2015; Snaidero et al., 2014; Takada et al., 2010).

To further validate the reduction in peripheral myelinating glial cells in erbb3b $b^{-/}$larvae, we quantified the number of $\mathrm{Sox}^{+} 0^{+}$glial cells along motor axons by performing immunohistochemistry with a Sox10 antibody to label glia, including ectopically-located 

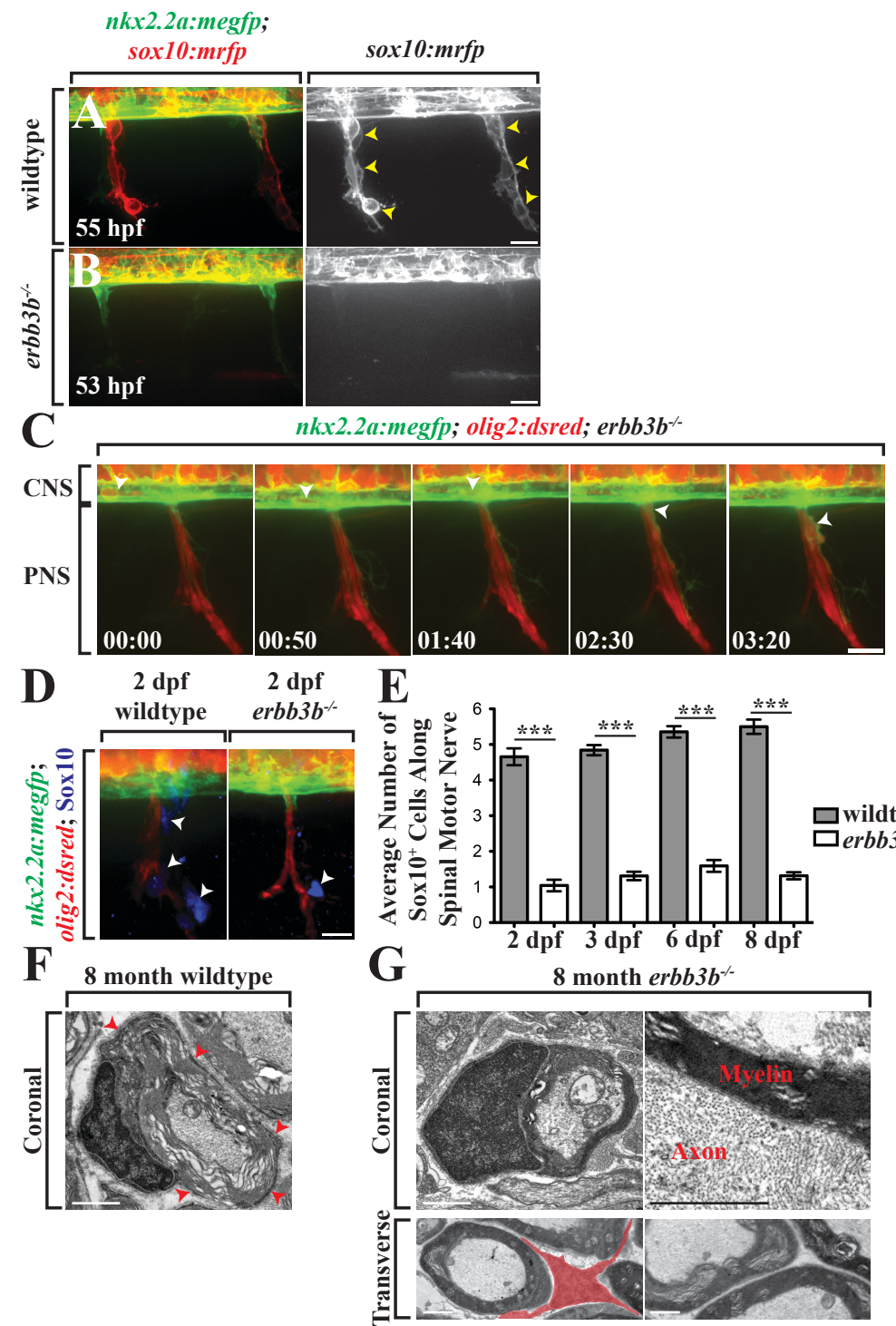

Figure 4-2. OPCs ectopically exit the spinal cord in $\boldsymbol{e r b b} 3 \boldsymbol{b}^{-/-}$larvae. Images in A - D are lateral views of the spinal cord with dorsal to the top and anterior to the left. (A) At $55 \mathrm{hpf}$ in $n k x 2.2 a$ :megfp;sox 10:mrfp larvae, $\operatorname{sox} 10^{+}$peripheral glial cells (arrowheads) associate with spinal motor axons in the periphery. (B) In contrast, sox $10^{+}$glial cells fail to associate with spinal motor axons in $n k x 2.2 a$ :megfp;sox10:mrfp;erbb3 $b^{-/}$ larvae at $53 \mathrm{hpf}$. (C) Frames captured from a 17 hour time-lapse movie of a $n k x 2.2 a: m e g f p$; olig2:dsred;erbb3 $3 b^{-/}$larva beginning at $57 \mathrm{hpf}$. Since the olig2:dsred transgene labels both motor axons and OPCs, an arrowhead is used to identify the OPC. An $n k x 2.2 a^{+} ;$olig2 $2^{+}$OPC (arrowhead) in the ventral spinal cord migrates towards the MEP TZ and ectopically exits the CNS. Numbers in the lower left corner identify the time that has elapsed from the first frame of the figure. (D) Lateral view of $2 \mathrm{dpf} n k x 2.2 a$;olig2:dsred wildtype and $e r b b 3 b^{-/}$larvae labeled with a Sox 10 antibody (blue, arrowheads). (E) Quantification of Sox $10^{+}$ glial cells along peripheral motor axons in wildtype and erbb3 $b^{-\leftarrow}$ larvae at 2, 3, 6 and $8 \mathrm{dpf}$. Sox $10^{+}$glial cells were significantly reduced in erbb3b ${ }^{-/}$larvae at all time points evaluated $(p<0.0001$ for all time points evaluated). Statistical significance was measured using an unpaired t-test. (F) Transmission electron micrograph of a coronal section identifying a peripheral myelinating glial cell that has myelinated an axon in an adult wildtype zebrafish motor nerve. Arrowheads denote presence of a basal lamina. (G) Transmission electron micrograph of coronal (top panels) and transverse (bottom panels) sections of adult erbb3bzebrafish spinal motor axons. Pseudocolor in the bottom left panel identifies the multi-polar processes of a cell, which may be an endoneurial cell or peripherally-located oligodendrocyte. Scale bars A - D, $25 \mu \mathrm{m}$. Scale bars F - G, $1 \mu \mathrm{m}$. 
OPCs, MEP glia and Schwann cells. Using nkx2.2a:megfp;olig2:dsred;erbb3b ${ }^{-/-}$or $n k x 2.2 a: m e g f p ; e r b b 3 b^{-/}$larvae co-labeled with an acetylated tubulin antibody, we were able to visualize spinal motor axons and associated Sox $10^{+}$glia (Figure 4-2D). We then selected developmental stages that are important for perineurial glial development, including $2 \mathrm{dpf}$, which corresponds to perineurial glia exit from the CNS, $3 \mathrm{dpf}$, which is when perineurial glia are extending along motor axons and 6 to $8 \mathrm{dpf}$, which is when perineurial glia begin to express ZO-1, and quantified the number of Sox $10^{+}$glia along individual spinal motor nerves (Binari et al., 2013; Kucenas et al., 2008b; Lewis \& Kucenas, 2014). At all ages assayed, we observed a significant reduction in the number of Sox $10^{+}$cells associated with erbb3 $3 b^{-/}$motor nerves ( 2 dpf, average $1.04, \mathrm{n}=121 ; 3$ dpf, average 1.31, $\mathrm{n}=110 ; 6 \mathrm{dpf}$, average $1.59, \mathrm{n}=110$; and 8 dpf, average $1.35, \mathrm{n}=$ $110)$ as compared to wildtype controls at similar developmental stages ( 2 dpf average $4.66, \mathrm{n}=176 ; 3$ dpf average $4.84, \mathrm{n}=176 ; 6$ dpf average $5.35, \mathrm{n}=110 ; 8$ dpf average $5.50, \mathrm{n}=110$ ) (Figure 4-2E). Previously, we demonstrated that Sox $10^{+}$cells along $e r b b 3 b^{-/}$spinal motor nerves are plpla $a^{+}$at $4 \mathrm{dpf}$ and express MBP at $8 \mathrm{dpf}$ (Smith et al., 2014). To further validate that ectopic oligodendrocytes are present along erbb3 $3 b^{-/}$ peripheral motor nerves, we examined spinal motor nerve ultrastructure in adult zebrafish using transmission electron microscopy. We observed myelinated spinal motor axons in both 8 month old wildtype and $e r b b 3 b^{-/}$zebrafish (Figure 4-2F - 4-2G, top panel). In a coronal cross section view of a wildtype zebrafish motor nerve, a distinct basal lamina was present around the myelin sheath, which is a defining feature of a Schwann cell (Figure 4-2F). However, there was no distinct basal lamina surrounding the myelin sheath in $\operatorname{erbb3} b^{-/}$nerves (Figure 4-2G). Furthermore, we observed Schwann cell processes 
associating with one axon in an adult wildtype zebrafish (Figure 4-2F). However, in $e r b b 3 b^{-/-}$motor nerves, we observed two morphologies of myelinating glial cells; single glial cell bodies were either associated with a single axon, reminiscent of a Schwann cell but lacking an organized basal lamina (Figure $4-2 \mathrm{G}$, top and bottom right panels), or multi-polar glial cell bodies with multiple processes were found associated with several axons, suggestive of an oligodendrocyte cell body (Figure 4-2G, bottom left panel). Therefore, the TEM data demonstrates ultrastructural characteristics that confirm myelinating glial cell changes along $e r b b 3 b^{-/}$motor nerves. Taken together, the alteration of peripheral myelinating glia, and the ectopic presence of OPCs in the mutant larvae, confirms that $e r b b 3 b^{-/}$spinal motor nerves are an ideal model for allowing us to assay the plasticity and resilience of perineurial glia in an altered developmental environment.

\section{Perineurial glia exit the spinal cord in the presence of ectopic oligodendrocytes}

Using $e r b b 3 b^{-/}$motor nerves as a model for investigating perineurial glial plasticity, we sought to determine if perineurial glial migration out of the spinal cord was affected in these mutants by conducting in vivo, time-lapse imaging experiments in nkx2.2a:megfp;sox 10:mrfp wildtype and erbb3 $b^{-/}$larvae. Perineurial glia begin exiting

the spinal cord at approximately $48 \mathrm{hpf}$ (Kucenas et al., 2008b). However, because zebrafish development proceeds in an anterior to posterior fashion, perineurial glia may not exit along some nerves until after $48 \mathrm{hpf}$. Based on our imaging location, between 52 hpf and $55 \mathrm{hpf}$ in wildtype larvae, we observed $n k x 2.2 a^{+}$perineurial glia migrate from the spinal cord and, once in the periphery, begin proliferating approximately 9.5 hours later (data not shown). Similarly, $n k x 2.2 a^{+}$perineurial glial migration and proliferation (data 
not shown) occurred in erbb3b $3 b^{-\alpha}$ larvae in a manner that was indistinguishable from wildtype larvae (data not shown). Intriguingly, $n k x 2.2 a^{+}$perineurial glia proliferation occurred in $e r b b 3 b^{-/-}$larvae in both the presence (Figure 4-3A) and absence of ectopic OPCs (Figure 4-3B).

Because perineurial glia migration from the $\mathrm{CNS}$ in $e r b b 3 b^{-/}$mutant larvae was indistinguishable from wildtype controls, we next investigated if they had stereotypic extension down developing spinal motor nerves. Using nkx2.2a:megfp;nbt:dsred transgenic lines to label perineurial glia and motor axons (Figure 4-4A), respectively, we imaged wildtype and $e r b b 3 b^{-/}$larvae and assayed perineurial extension by measuring the length of GFP expression from the MEP TZ to the horizontal myoseptum, a partition of connective tissue located between the dorsal and ventral body wall muscles. Although not statistically significant, we observed a consistent reduction in the extension length of perineurial glia in $e r b b 3 b^{-/}$larvae at all developmental stages evaluated (data not shown), demonstrating that perineurial glia are capable of migrating and extending along motor nerves in the absence of all peripheral myelinating glia and in the presence of ectopic OPCs.

Although the perineurial extension length was only slightly reduced in $e r b b 3 b^{-/}$larvae as compared to wildtype controls, we observed distinct morphological differences in perineurial glia in $e^{2} b b 3 b^{-/}$larvae at $8 \mathrm{dpf}$. We categorized these morphological observations into the following categories: 1) $n k x 2.2 a^{+}$perineurial glia with ensheathment comparable to wildtype, 2) patchy or discontinuous populations of perineurial glia, 3) 
A
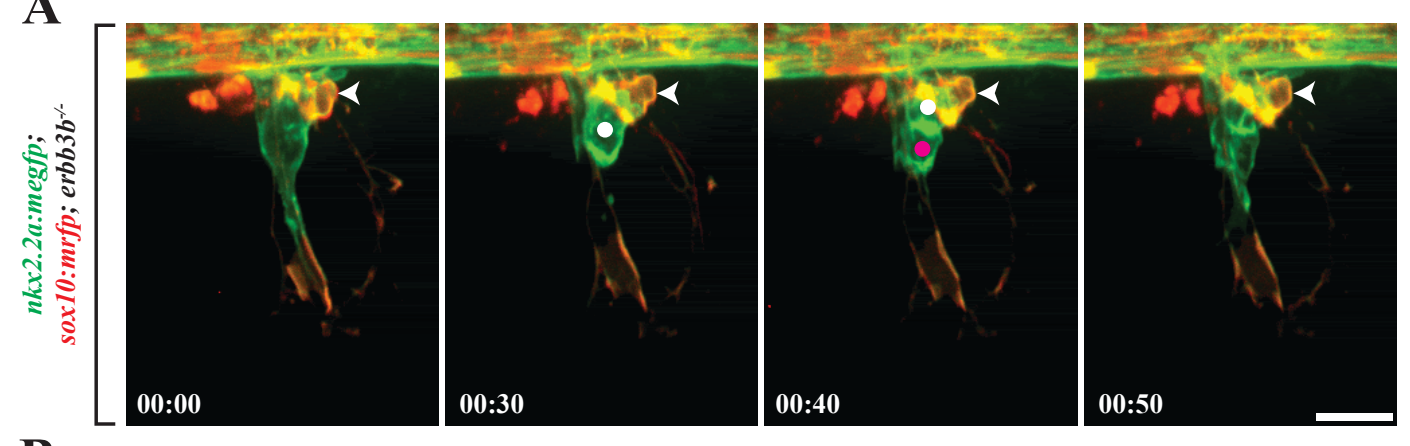

B
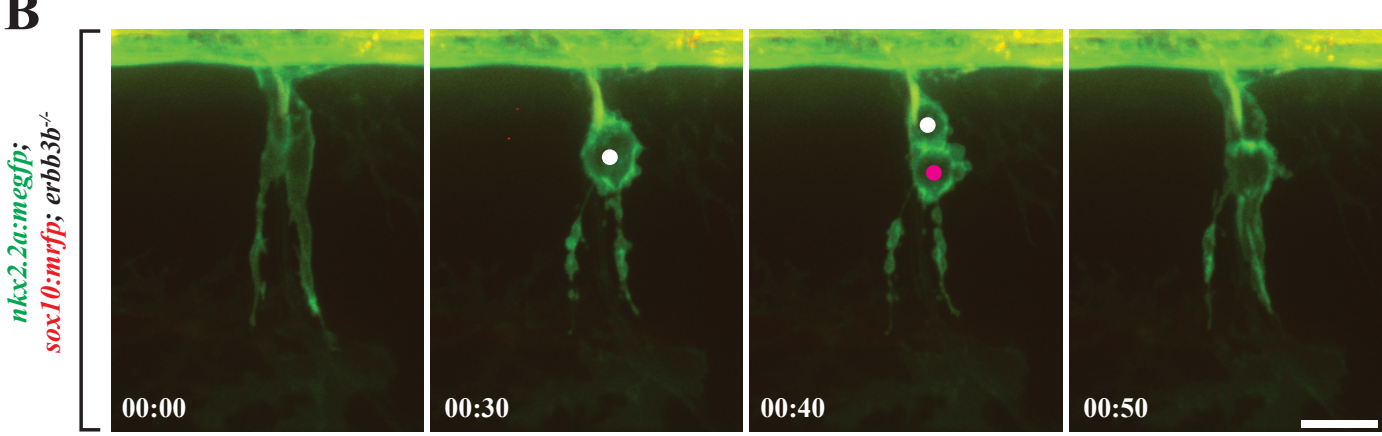

Figure 4-3. Perineurial glial proliferation is normal in $\boldsymbol{e r b b} \mathbf{b} \boldsymbol{b}^{-/}$larvae. All images are lateral views of the spinal cord with dorsal to the top and anterior to the left. (A) Frames captured from a 16 hour time-lapse movie of a $55 \mathrm{hpf} n k x 2.2 a$ :megfp;sox 10:mrfp; $\mathrm{erbb}^{-/ \sim}$ larva reveals that perineurial glial proliferation (dots) occurs despite the presence of an ectopically-located OPC (arrowhead). (B) Frames captured from a 13 hour time-lapse movie of a 59 hpf $n k x 2.2 a$ :megfp;sox10:mrfp;erbb3b-^ larva reveals that perineurial glial proliferation (dots) also occurs in the absence of ectopically-located OPCs. Numbers in the lower left corner identify the time that has elapsed from the first frame of the figure. Scale bars, $25 \mu \mathrm{m}$. 
perineurial glia that were thin and/or tubular and 4) perineurial cells that were located only at the MEP TZ (Figure 4-4A). In 8 dpf wildtype larvae, we observed the percentage of motor nerves with the following perineurial glial morphologies: $42 \%$ were fully ensheathed (26 out of 61 nerves), $8 \%$ had nerves with patchy / discontinuous streams of cells ( 5 out of 61 nerves), 25\% had perineurial cells that were thin / tubular (15 out of 61 nerves) and 25\% had perineurial glia located only at the MEP TZ (15 out of 61 nerves) (Figure 4-4A - 4-4B). In contrast, the morphology of perineurial glia in $8 \mathrm{dpf} e r b b 3 b^{-1-}$ larvae was: $20 \%$ with full ensheathment (12 out of 60 nerves), $10 \%$ patchy / discontinuous (6 out of 60 nerves), 47\% with thin / tubular morphology (28 out of 60 nerves) and 23\% located only at the MEP TZ (14 out of 60 nerves) (Figure 4-4A - 4-4B). Thus, in 8 dpf wildtype larvae, there was a greater percentage of motor nerves with full perineurial glial ensheathment whereas in $8 \mathrm{dpf} e r b b 3 b^{-\alpha}$ larvae, there was a greater percentage of motor nerves with thin / tubular perineurial glial morphology.

Next, we investigated the ultrastructure of the perineurium using transmission electron microscopy in adult wildtype and erbb3 $3 b^{-/}$zebrafish. In both wildtype and erbb3 $3 b^{-/}$ adults, we observed myelinated axons bundled into nerve fascicles by the perineurium (Figure 4-4C). However, in erbb3b $b^{-/}$zebrafish, the morphology of the perineurium varied. There were areas of the perineurium in which the ultrastructural morphology appeared comparable to wildtype, as we observed concentric and compacted perineurial layers in both wildtype (Figure 4-4D) and mutants (Figure 4-4E, left panel). However, there were other areas of the mutant perineurium that appeared very disorganized (Figure 4-4E, right panel), i.e., the layers lacked compaction, were discontinuous and had a wavy 

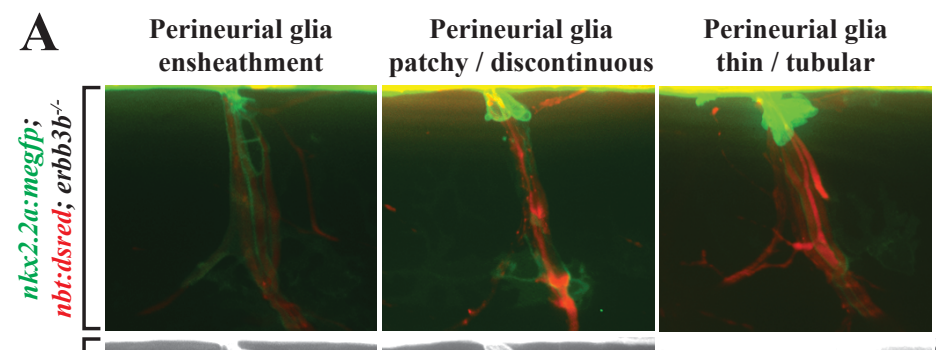

Perineurial glia
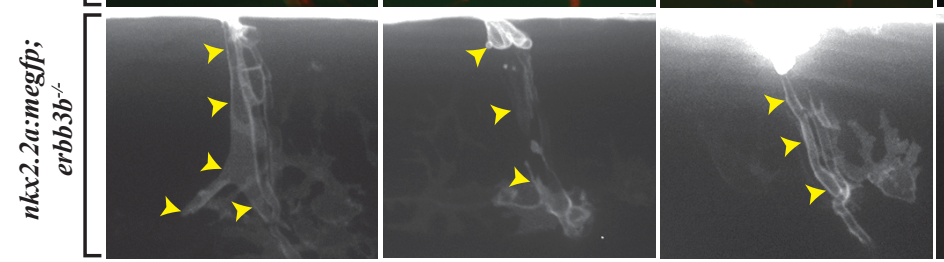

at MEP TZ

B

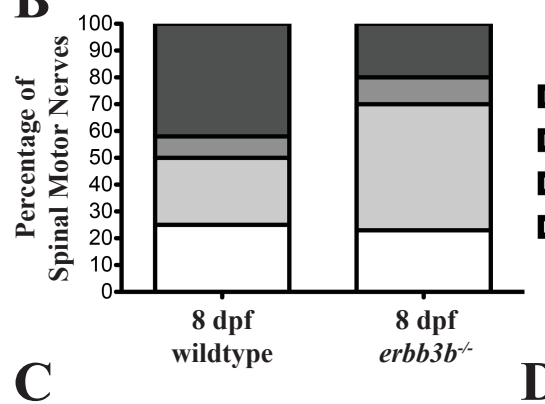

물ineurial glia ensheathment

Derineurial glia patchy / discontinuous

$\square$ Perineurial glia thin / tubular

$\square$ Perineurial glia at MEP TZ

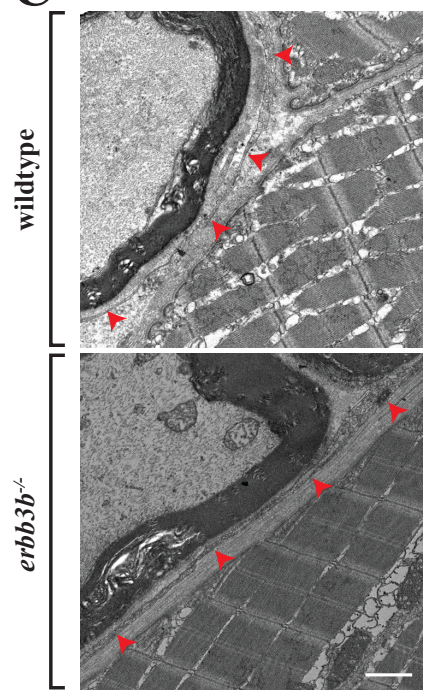

D

E

E
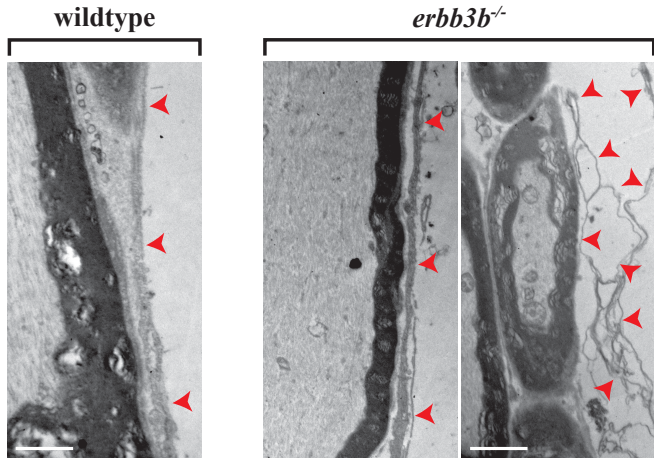

Figure 4-4. Perineurial glial morphology is altered in $\boldsymbol{e r b}_{\boldsymbol{b}} 3 \boldsymbol{b}^{--}$zebrafish. (A) Images are lateral views of the spinal cord with dorsal to the top and anterior to the left. Morphologically, $n k x 2.2 a^{+}$perineurial glia (arrowheads) in $8 \mathrm{dpf}$ erbb $3 b^{-/}$were different from wildtype and we classified them into the following categories: perineurial glia ensheathment comparable to wildtype, perineurial glia that appeared patchy or discontinuous, perineurial glia thin / tubular and perineurial glia only at the MEP TZ. Motor axons (red) are labeled using the $n b t$ :dsred transgene. (B) Quantification of $n k x 2.2 a^{+}$perineurial glial morphology along spinal motor nerves in $8 \mathrm{dpf}$ wildtype and erbb3 $b^{-/}$larvae. (C) Transmission electron micrographs of coronal cross sections of 8 month old wildtype zebrafish perineurium (top, arrowheads) and 8 month old erbb3 $b^{-/}$ zebrafish perineurium (bottom, arrowheads). (D) Transmission electron micrograph of a transverse section of an adult wildtype perineurium (arrowheads). (E) Transmission electron micrograph of a transverse section of an adult $e r b b 3 b^{-/}$perineurium demonstrates there are areas of the perineurium (left panel, arrowheads) that appear comparable to wildtype in (D), and there are areas of the erbb3 $b^{-/}$perineurium that appear disorganized (right panel, arrowheads). Scale bar A, $25 \mu \mathrm{m}$. Scale bar C - E, $1 \mu \mathrm{m}$. 
appearance, and we never observed these phenotypes in wildtype animals. Taken together, these results demonstrate that although certain perineurial glial migration and proliferation behaviors are comparable to wildtype larvae, perineurial glial morphology is altered in mutants with ectopic OPCs along spinal motor nerves.

\section{Ectopically-located OPCs affect perineurial glial response to injury}

Although perineurial glial migration from the CNS is indistinguishable from wildtype larvae in mutants with ectopic OPCs, we wanted to investigate whether they were capable of responding to injury despite this change in cellular and myelin composition along spinal motor nerves. Peripheral nerves have the remarkable capacity to regenerate following nerve transection, whereby the nerve is divided and a regenerative program is induced, triggering Wallerian degeneration (Waller, 1850), debris clearance and the regrowth of axons from the proximal stump back to peripheral targets (Arthur-Farraj et al., 2012; Rosenberg et al., 2014). Previously, we identified perineurial glia as an essential component of the injury response (Lewis \& Kucenas, 2014). After motor nerve transection, perineurial glia closest to the injury area extend processes toward the injury site, phagocytize debris and form the first tissue bridge across the injury gap (Lewis \& Kucenas, 2014). Recently, a study demonstrated that axonal regrowth was significantly impaired in $e r b b 3 b^{-/}$larvae following injury (Rosenberg et al., 2014), and we confirmed this finding in our assay of motor nerve transection as well (data not shown). Due to the impairment of axonal regrowth in erbb3b $b^{-/}$larvae, coupled with the presence of perineurial glia in the periphery, we hypothesized that perineurial glia did not perform important regenerative roles, including extending toward the injury site, forming 
phagocytic vesicles and bridging injury gaps, leading to the failure of axonal regeneration in these mutants.

To test this hypothesis, we investigated whether perineurial glia were capable of extending towards an injury site in the presence of peripheral OPCs by selecting wildtype and $e r b b 3 b^{-/}$motor nerves that lacked full ensheathment by $n k x 2.2 a^{+}$perineurial glia, and mutant axonal tracts that also contained ectopically-located OPCs. Using the motor nerve transection assay we described previously (Lewis \& Kucenas, 2013; 2014) in 4 dpf nkx2.2a:megfp;olig2:dsred larvae, we transected a region of unensheathed motor nerve on the caudal motor tract approximately 10 to $15 \mu \mathrm{m}$ from the closest perineurial glial cell. As we previously reported, we observed perineurial glia in wildtype larvae rapidly extend highly motile membrane processes toward the injury site (Figure 4-5A). By 3 hours post transection (hpt), these processes had extended beyond the initial injury site and stretched toward the distal stump (Figure 4-5A) (Lewis \& Kucenas, 2014). In $e r b b 3 b^{-/}$larvae, perineurial glia initially extended membrane processes toward the injury site. However, in contrast to wildtype larvae, these processes eventually stalled, retracted or became misdirected and failed to reach the injury even by $3 \mathrm{hpt}$ (Figure 4-5B). We quantified these data by plotting perineurial membrane extension towards the injury site as a percentage of the initial distance between the injury site and the closest perineurial glial membrane (i.e., distance traveled toward injury site / initial distance between perineurial glia and injury site), with a value of 1 representing perineurial processes reaching the injury site, a value of $>1$ representing perineurial processes with extension beyond the injury site and values $<1$ representing perineurial processes that did not reach 
the injury site within the time investigated post injury. In erbb3b $b^{-/}$larvae, we observed that perineurial process extension toward injury sites was significantly reduced by 120 minutes post transection (mpt) as compared to wildtype, demonstrating a failure of membrane extension across the injury site (Figure 4-5C) (Lewis \& Kucenas, 2014). Surprisingly, the initial response $(15 \mathrm{mpt}$ to $1 \mathrm{hpt})$ of perineurial glia to the injury in $e r b b 3 b^{-/-}$larvae was indistinguishable from wildtype larvae, demonstrating that perineurial glia in mutant larvae are capable of initially responding to injury cues. From these data, we conclude that ectopically-located OPCs impair the maintained extension of perineurial processes to an injury (Lewis \& Kucenas, 2014).

Because perineurial glial process extension towards the injury site is impaired after transection of the caudal axonal tract containing ectopically-located OPCs, we decided to injure the rostral axonal tract, which we never observe populated by ectopic OPCs, to investigate if perineurial-perineurial interactions were perturbed in the absence of all myelinating glial cells. Specifically, we investigated whether perineurial glia were capable of phagocytizing debris and forming a glial bridge across injury sites along $e r b b 3 b^{-/-}$nerves (Lewis \& Kucenas, 2014). We selected motor nerves that were ensheathed by perineurial glia but lacked ectopically-located OPCs, and transected the rostral motor nerve tract in $6 \mathrm{dpf} n k x 2.2 a$ :megfp;olig2:dsred wildtype and erbb3b $b^{-/}$ larvae (Lewis \& Kucenas, 2013; 2014). Following transection, we assayed the perineurial response and, similar to our previous findings, we observed wildtype perineurial glia located proximally and distally to the injury site respond to the injury by extending processes toward the lesion, form phagocytic vesicles and bridge the injury gap (Figure 


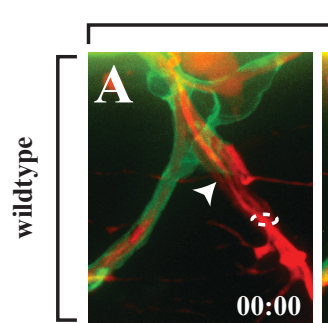

nkx2.2a:megfp; olig2:dsred
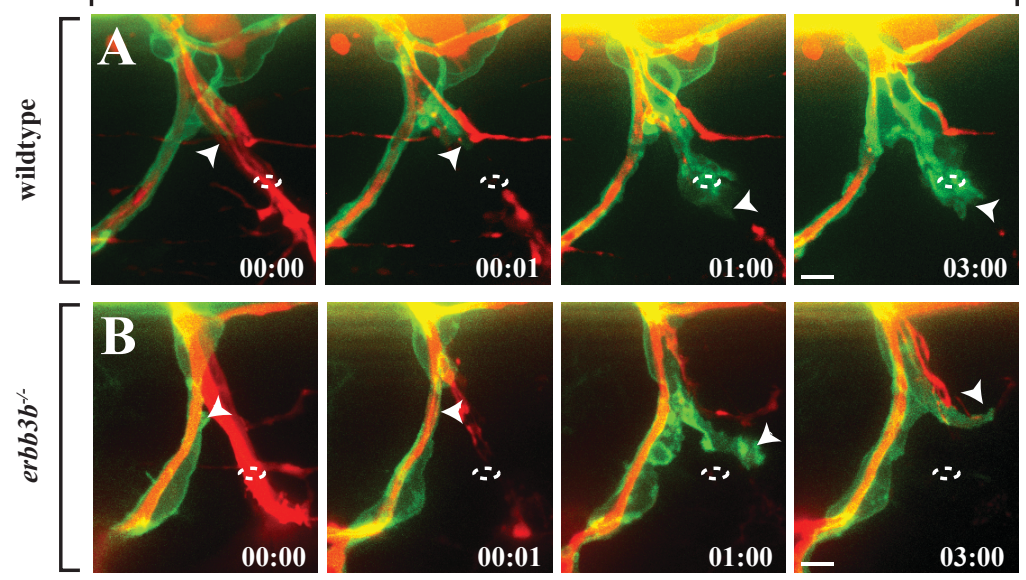

C

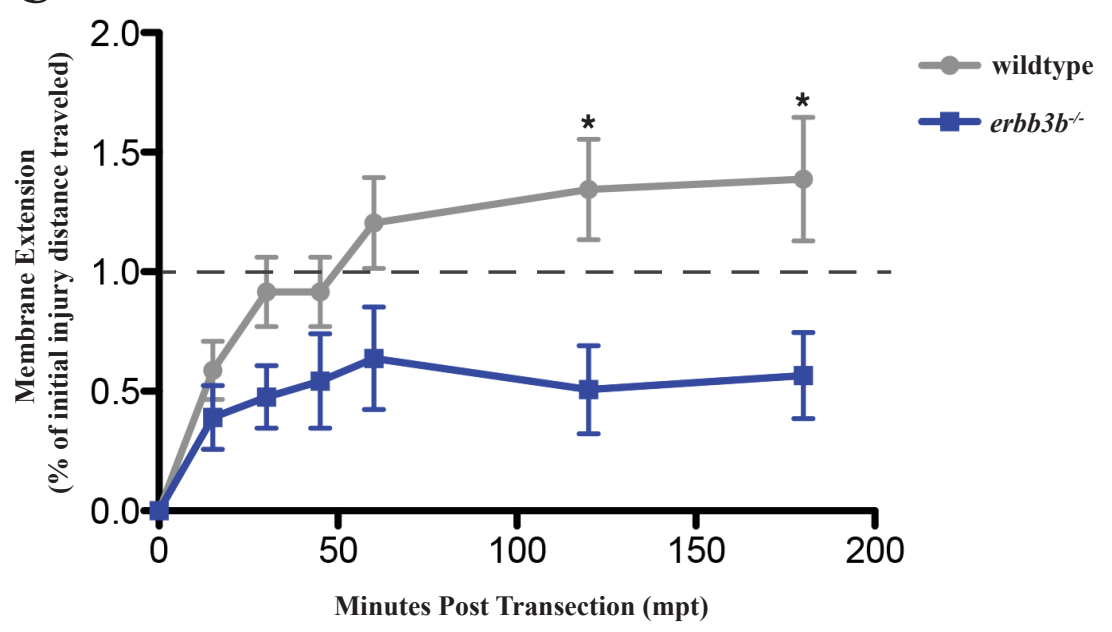

Figure 4-5. Perineurial glial recruitment to the injury site is impaired on axonal tracts with centrally-derived OPCs. All images are lateral views of the spinal cord with dorsal to the top and anterior to the left. (A) Frames captured from a 3 hour time-lapse movie of a 4 dpf $n k x 2.2 a$ :megfp; olig2:dsred wildtype larva. After axotomy, perineurial glia (arrowheads) extended processes into the injury site following motor nerve transection. (B) In contrast, in frames captured from a 3 hour time-lapse movie of a $4 \mathrm{dpf} n k x 2.2 a$ :megfp; olig2:dsred;erbb3b- larva, perineurial glia (arrowheads) initially extended robust processes toward the injury site following transection of an axonal tract containing ectopically-located OPCs. However, the processes failed to reach the injury. Numbers in the lower right corner denote time elapsed (mpt) from the initial panel of the figure, which was immediately prior to axotomy. (C) Quantification of the extension of perineurial processes toward transection sites in $4 \mathrm{dpf}$ wildtype and $e r b b 3 b^{-/}$larvae shows perineurial glia extend processes significantly farther in wildtype by $120 \mathrm{mpt}(\mathrm{p}=0.0132)$ and $180 \mathrm{mpt}(\mathrm{p}=0.0478)$. Because the initial distance between the injury site and glial cells varied slightly with each trial, membrane extension was plotted as a percentage of the initial distance between the injury site and the closest perineurial glial membrane (distance traveled toward injury site / initial distance between perineurial glia and injury site), where values greater than 1 represent processes that have extended beyond the initial injury site. Dotted ellipse denotes approximate ablated ROI. Scale bar, $25 \mu \mathrm{m}$. 
$4-6 \mathrm{~A})$ in $90 \%$ of cases $(\mathrm{n}=10)$ (Lewis \& Kucenas, 2014). Intriguingly, the perineurial response in $e r b b 3 b^{--}$larvae was similar (Figure 4-6B). Perineurial glia extended processes towards the injury site from both the proximal and distal stumps, formed phagocytic vesicles and bridged the injury gap (Figure $4-6 \mathrm{~B})$ in $75 \%$ of cases $(n=4$, not significantly different from wildtype). Taken together, results from these injury studies demonstrate that the presence of ectopic OPCs and central myelin affect perineurial glial responses to injury, but that peripheral myelinating glia are not required for perineurial glia to clear debris and form a glial bridge.

\section{Perineurial glia have an increase in ZO-1 labeling along $e^{2} b b 3 b^{-/}$spinal motor nerves}

In addition to developmental and injury response roles, perineurial glia maintain and protect the nerve by maturating into a component of the blood-nerve barrier. A hallmark of perineurial differentiation is the formation of tight junctions between adjacent perineurial cells, which is required for the mature permeability function of this structure (Clark et al., 2014; Kristensson \& Olsson, 1971; Kucenas et al., 2008b; Pummi et al., 2004). In zebrafish, ZO-1 labeling of tight junctions along spinal motor nerves is evident at $6 \mathrm{dpf}$ beginning at the MEP TZ. As development proceeds, ZO-1 labeling is observed further distally along the nerve and this is a sign of progressive perineurial differentiation (Kucenas et al., 2008b; Lewis \& Kucenas, 2014). Intriguingly, a previous study demonstrates that oligodendrocytes are involved in supporting the integrity of the bloodbrain barrier (BBB) by increasing the formation of tight junctions and expression of ZO-1 within BBB components (Seo et al., 2014). Because $e r b b 3 b^{-/}$larvae have ectopically- 
nkx2.2a:megfp; olig2:dsred

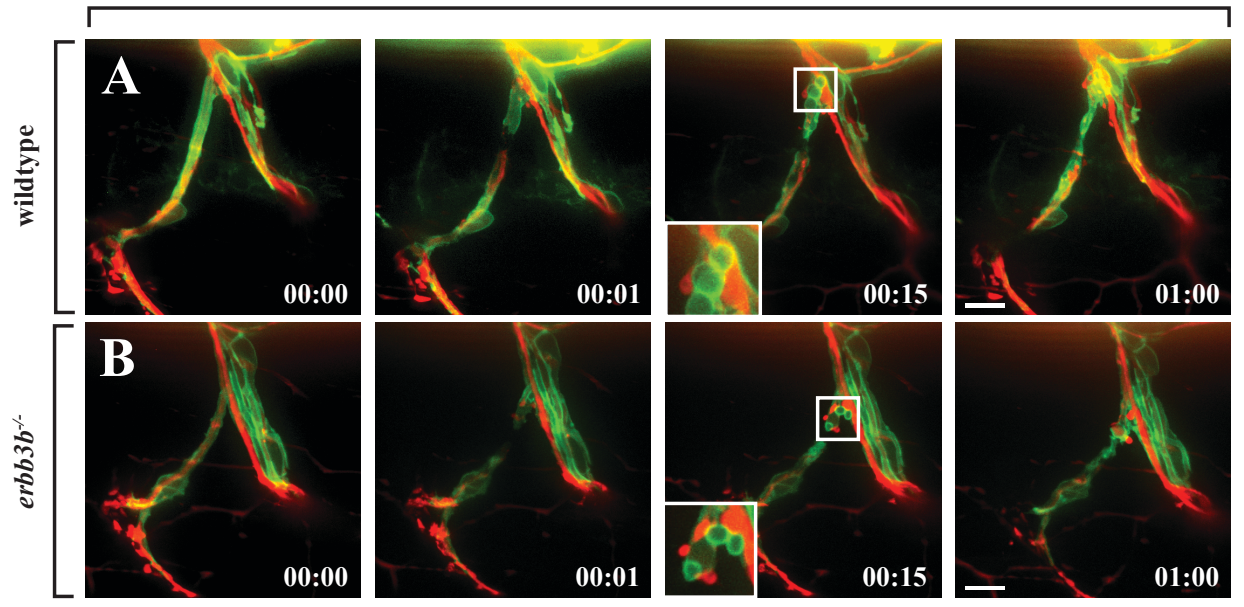

Figure 4-6. Perineurial glia form phagocytic vesicles and glial bridges in the absence of all myelinating glial cells. All images are lateral views of the spinal cord with dorsal to the top and anterior to the left. (A) Frames captured from 1 hour of a 3 hour time-lapse movie of a $6 \mathrm{dpf}$ nkx2.2a:megfp;olig2:dsred wildtype larva demonstrate that perineurial glia form phagocytic vesicles and glial bridges that span the injury site following axotomy. (B) Similarly, frames captured from a 3 hour time-lapse movie in a $6 \mathrm{dpf} n k x 2.2 a$ :megfp;olig2:dsred;erbb3b ${ }^{-/}$larva show that perineurial glia form phagocytic vesicles and glial bridges that span the injury site in the absence of all myelinating glia. Numbers in lower right corners denote time elapsed (mpt) from the initial panel of the figure, which was immediately prior to axotomy. Scale bars, $25 \mu \mathrm{m}$. 
located OPCs along spinal motor nerves, we hypothesized that their presence in the periphery may impact perineurial glial development by altering ZO-1 expression. Using an antibody to ZO-1 in 6 and 8 dpf larvae, we labeled fixed tissue, imaged motor nerves in both $n k x 2.2 a: m e g f p$ wildtype (Figure 4-7A) and $n k x 2.2 a: m e g f p ;$; $r b b 3 b^{--}$(Figure 4-7B) larvae and assayed the extension of perineurial tight junctions by measuring the length of ZO-1 labeling along spinal motor nerves, starting from the MEP TZ. From these studies, we observed no statistically significant difference in ZO-1 extension at 6 dpf (data not shown). However, by $8 \mathrm{dpf}$, we observed a significant increase in the extension of perineurial glial ZO-1 labeling along $\operatorname{erbb} 3 b^{-/}$nerves with ectopic OPCs (average $=$ $63.83 \mu \mathrm{m}, \mathrm{n}=61$ nerves) when compared to wildtype (average $=45.62 \mu \mathrm{m}, \mathrm{n}=61$ nerves) and $e r b b 3 b^{-/}$nerves without ectopic OPCs (average $=36.47, \mathrm{n}=5$ nerves) (Figure 4-7C).

To determine if ZO-1 labeling was also enhanced in motor nerves of $e r b b 3 b^{-/ .}$adults, we labeled coronal sections of $n k x 2.2 a: m e g f p$;sox10:mrfp erbb3b $b^{-/}$and wildtype adults ( $>3$ months old) with an antibody to ZO-1 (Figure 4-7D - 4-7E). In these studies, we observed abnormally large deposits of ZO-1 immunoreactivity within $n k x 2.2 a^{+}$ perineurial layers around $e r b b 3 b^{-/}$nerves (Figure 4-7E) as compared to wildtype controls, which appeared to have more diffuse ZO-1 labeling in the perineurium (Figure 4-7D). To quantify this observation, we measured the fluorescence intensity of ZO-1 labeling within six $n k x 2.2 a^{+} / \mathrm{ZO}-1^{+}$regions of the perineurium in both wildtype and $e r b b 3 b^{-/}$nerves and, after correcting for background fluorescence, analyzed the average corrected fluorescence intensity of each ROI in both groups (Figure 4-7F). We observed a 
statistically significant increase in the average ROI corrected fluorescence intensity of ZO-1 labeling in the perineurium in adult $e r b b 3 b^{-/}$nerves (average $=28189$ A.U., $\mathrm{n}=54$ ROIs) as compared to wildtype controls (19469 A.U., $\mathrm{n}=30$ ROIs) (Figure 4-7F). Since we observed large deposits of ZO-1 labeling within the perineurium of erbb3 $3 b^{-/}$adults, we next categorized the corrected fluorescence intensity for each ROI into one of the following: 0 - 25000 A.U., 25001 - 50000 A.U. or 50001 - 75000 A.U. (Figure 4-7G). In wildtype controls, we observed the majority of ROIs were within the lowest fluorescence intensity category: $73 \%$ of ROIs fall within 0 - 25000 A.U. (22 / 30 ROIs), $23 \%$ of ROIs (7 / 30 ROIs) were within 25001 - 50000 A.U. and 3\% of ROIs (1 / 30 ROIs) were within the highest fluorescence intensity category of $50001-75000$ A.U. In contrast, the $e r b b 3 b^{-/}$ROIs had the following distribution: 52\% (28 / 54 ROIs) were within $0-25000$ A.U., 41\% (22 / 54 ROIs) within 25001 - 50000 A.U. and 7\% (4 / 54 ROIs) within 50001 - 75000 A.U, demonstrating that there were patches of significantly higher tight junctional proteins. To confirm that the less diffuse and increased level of ZO-1 labeling we observed within the $e r b b 3 b^{-/}$perineurium was due to an increase in tight junctions, we performed transmission electron microscopy to investigate the perineurium ultrastructure in 8 month old wildtype and mutant zebrafish. We observed tight junctions in both wildtype and $e r b b 3 b^{-/}$zebrafish (Figure 4-7H). However, there were discrete areas of the perineurium that had clusters of more tight junctions present within the mutant perineurium (Figure 4-7H, right panel).

Because of the altered distribution of tight junctions observed in $e r b b 3 b^{-/}$adults, we investigated the barrier function of this structure by injecting a fluorescent dextran dye 

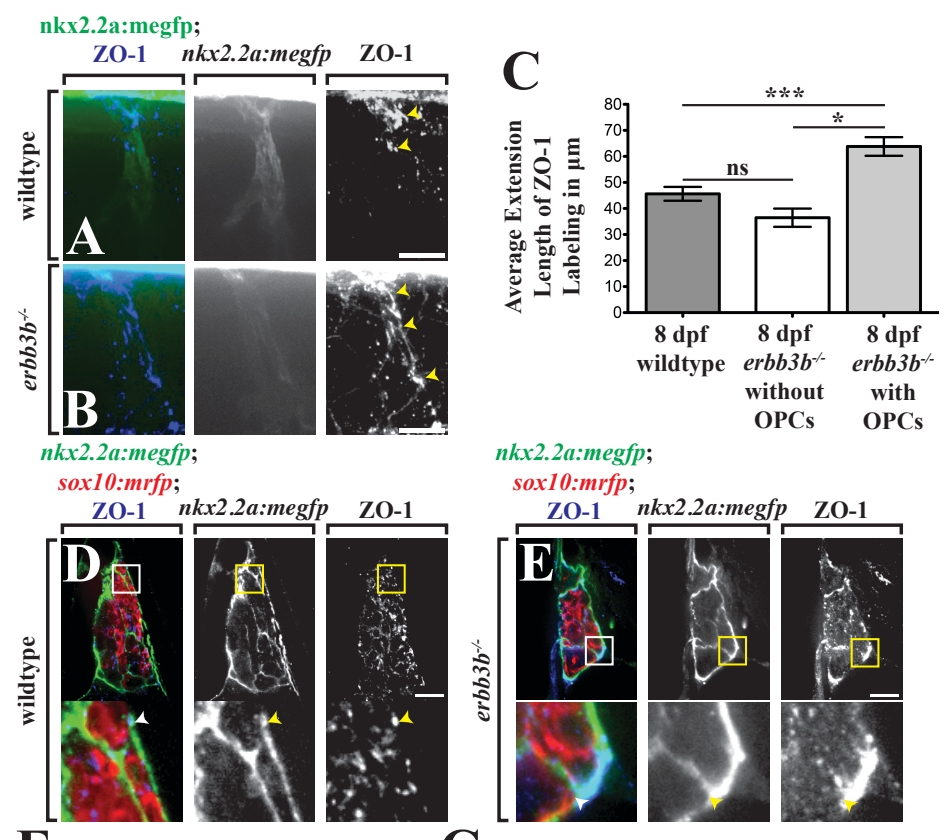

sox10:mrfp;
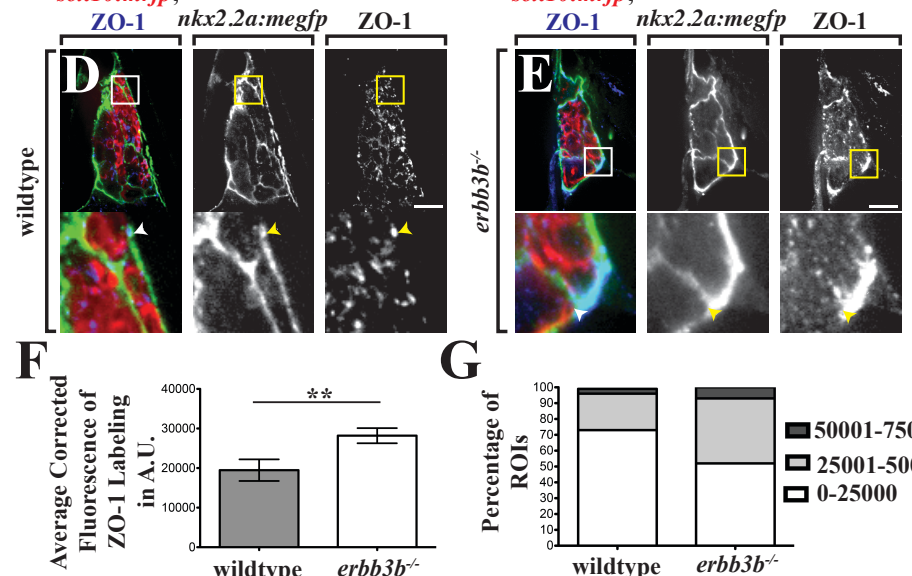

$\mathbf{H}$

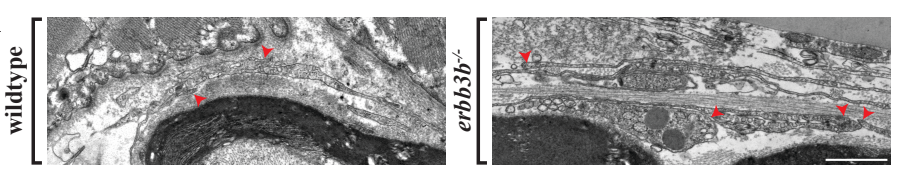

Figure 4-7. Perineurial glia have more tight junctional proteins in erbb3b mutant zebrafish. (A \& B) Lateral views of $8 \mathrm{dpf} n k x 2.2 a$ :megfp wildtype and $e r b b 3 b^{-\digamma}$ larvae, with dorsal to the top and anterior to the left, labeled with an antibody to ZO-1 (blue). (A) At 8 dpf, ZO-1 labeling (arrowhead) is primarily localized near the MEP TZ in wildtype larvae. (B) In $8 \mathrm{dpf} e r b b 3 b^{--}$larvae, ZO-1 labeling (arrowhead) extended further ventrally towards the horizontal myoseptum. (C) Quantification of the extension length of ZO-1 labeling along peripheral motor nerves in $8 \mathrm{dpf} n k x 2.2 a: m e g f p$ wildtype and $e r b b 3 b^{-/}$larvae. The ZO-1 labeling along erbb3 $b^{-/}$motor nerves that contain ectopically-located OPCs extended significantly further towards the horizontal myoseptum as compared to $8 \mathrm{dpf}$ wildtype larvae $(\mathrm{p}<0.0001)$ and $8 \mathrm{dpf}$ erbb $3 b^{-1}$ motor nerves that did not contain OPCs $(\mathrm{p}=0.0339)$. Statistical significance was measured using an unpaired t-test. (D\&E) Coronal cross sections of peripheral motor nerves in adult nkx2.2a:megfp;sox10:mrfp wildtype and $e r b b 3 b^{-\varsigma}$ zebrafish labeled with an antibody to ZO-1 (blue). (D) ZO-1 labeling (arrowhead) appeared more punctate within wildtype perineurium. (E) In contrast, ZO-1 labeling (arrowhead) in erbb3 $3 b^{-/}$adult perineurium was more heavily concentrated in discrete regions. (F) Quantification of the average fluorescence intensity, corrected for background, of ZO-1 labeling in Arbitrary Units (A.U.) within $n k x 2.2 a^{+} / \mathrm{ZO}-1^{+}$ROIs in adult $n k x 2.2 a: m e g f p ; s o x 10: m r f p$ wildtype and erbb3b $b^{-/}$zebrafish. Six ROIs were selected per nerve (wildtype $\mathrm{n}=5$ nerves; $\operatorname{erbb}^{-} b^{-\sim} \mathrm{n}=9$ nerves). There was a statistically significant increase in the average corrected fluorescence intensity of ZO-1 labeling within the erbb3 $3 b^{-/}$adult perineurium ROIs ( $\mathrm{n}=54$ ROIs) as compared to wildtype control ROIs ( $\mathrm{n}=30$ ROIs) $(\mathrm{p}=0.0094)$. $(\mathrm{G})$ Categorization of the corrected fluorescence intensity of the ROIs. (H) Transmission electron micrograph of a coronal cross section of an adult wildtype (left panel) and an adult erbb3 $3 b^{-\curvearrowright}$ (right panel) perineurium identifying tight junctions (arrowheads). Scale bars A, B, D \& E, $25 \mu \mathrm{m}$. Scale bar H, $1 \mu \mathrm{m}$. 
into the muscle of adult WT and erbb3b mutants. Fluorescent dextrans have previously been used in mammals to investigate the barrier function of the blood-brain barrier and choroid plexus epithelial cells (Ek et al., 2003; Liddelow et al., 2009; Saunders et al., 2015). Therefore, we injected a 10,000 MW Dextran-647 into the muscle of 8 month old wildtype and $e r b b 3 b^{-/}$zebrafish and fixed harvested trunk tissue at 1.5 hours post injection (hpi). We imaged coronal cross sections and observed that the Dextran-647 remained localized to the nerve perimeter in wildtype adults, demonstrating the perineurial barrier was functional (Figure 4-8A). However, in $e r b b 3 b^{-/}$adults, we observed Dextran-647 infiltration into the nerve, demonstrating that the integrity of the perineurial barrier was compromised (Figure 4-8B). Taken together, these results demonstrate that the increase in ZO-1 labeling observed along spinal motor nerves in 8 dpf $e r b b 3 b^{-\alpha}$ larvae is redistributed into clusters of tight junctions within the $e r b b 3 b^{-/}$ adult perineurium, and although clusters of tight junctions are present, the barrier function of the $e r b b 3 b^{-/}$perineurium is compromised.

\section{TGF-p1 partially mediates the increase in ZO-1 labeling along erbb3b ${ }^{-/}$motor}

\section{nerves}

A previous study implicated OPCs in promoting the formation of tight junctions in the BBB via TGF- $\beta 1$, as depleting TGF- $\beta 1$ specifically in OPCs decreased ZO-1 labeling in this tissue (Seo et al., 2014). Upon ligand binding, the TGF- $\beta$ type II receptor phosphorylates the type I receptor (Kitisin et al., 2007). The activated type I receptor then

phosphorylates Smad effector proteins, initiating the modulation of gene expression (Kitisin et al., 2007; Sun et al., 2006). Since TGF- $\beta 1$ is important for promoting ZO-1 


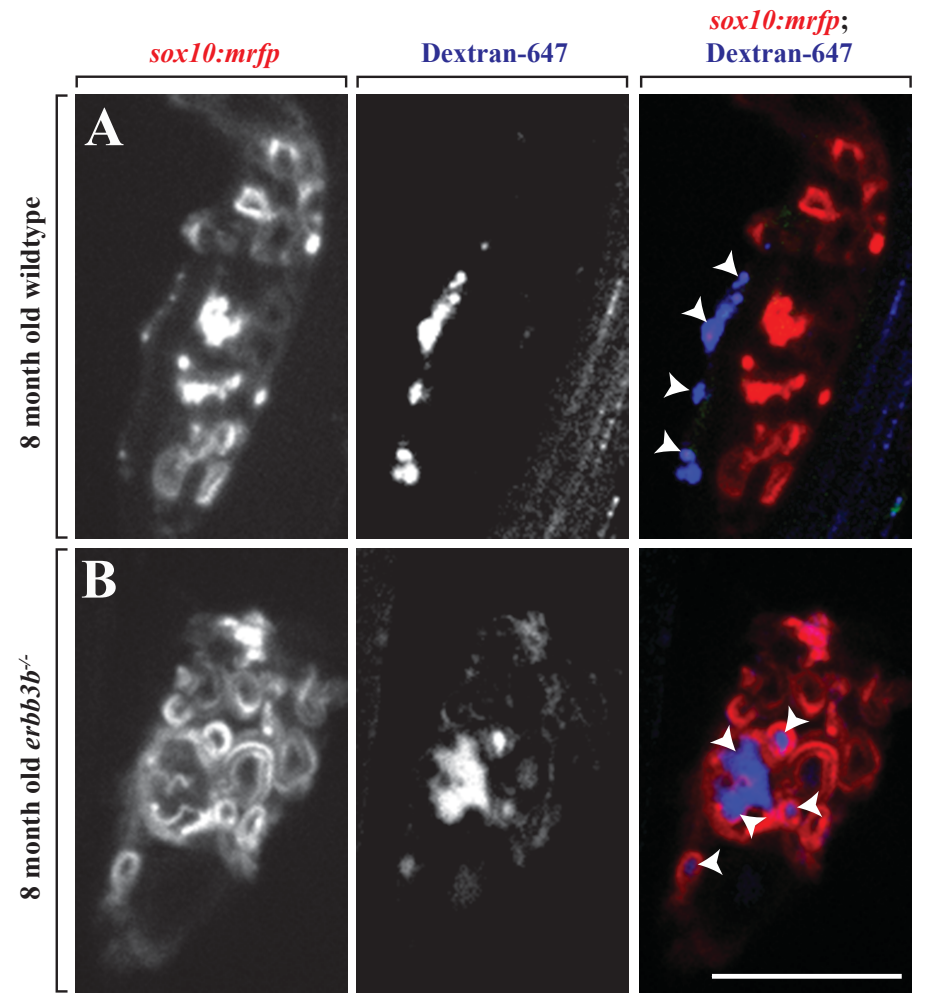

Figure 4-8. Perineurial barrier integrity is compromised in adult $\operatorname{erbb} 3 \boldsymbol{b}^{-\sim}$ zebrafish.All images are cross sections of 8 month old sox 10:mrfp wildtype and erbb3 $b^{-/}$zebrafish injected with a Dextran-647 dye (blue) into the muscle. (A) In wildtype, the Dextran-647 dye (blue, arrowheads) remains localized to the perimeter of the motor nerve. (B) In erbb3 $3 b^{--}$, the Dextran-647 dye (blue, arrowheads) infiltrates into the motor nerve. Scale bar, $25 \mu \mathrm{m}$. 
expression in the BBB, we hypothesized that TGF- $\beta 1$ signaling would be present along peripheral spinal motor nerves in zebrafish larvae.

To investigate this hypothesis, we fixed $n k x 2.2 a$ :megfp; ${\text { erb } b 3 b^{-/}}^{-}$and wildtype control larvae at $8 \mathrm{dpf}$ and labeled with an antibody to phosphorylated Smad3 (pSmad3), a downstream effector of TGF- $\beta 1$ signaling (Kitisin et al., 2007). We observed specific pSmad3 labeling along $68.18 \%$ of wildtype spinal motor nerves $(\mathrm{n}=66$ nerves) (Figure $4-9 \mathrm{~A}-4-9 \mathrm{C})$ and $63.6 \%$ of $e r b b 3 b^{-/}$spinal motor nerves $(\mathrm{n}=66$ nerves) (Figure 4-9B 4-9C) at 8 dpf. Although the percentage of nerves that were $\mathrm{pSmad}^{+}$was not significantly different between mutant and wildtype larvae at this stage, we observed more robust pSmad3 labeling along erbb3 $3 b^{-/}$larval motor nerves when compared to wildtype. Therefore, we categorized pSmad3 expression along motor nerves as strong, moderate, low and none (Figure 4-9A - 4-9B). In wildtype larvae, we observed 13.64\% ( 9 out of 66 nerves) with strong pSmad3 expression, 13.64\% (9 out of 66 nerves) with moderate pSmad3 expression, 40.91\% (27 out of 66 nerves) with low pSmad3 expression and $31.82 \%$ (21 out of 66 nerves) with no pSmad3 expression (Figure 4-9C). Along $e r b b 3 b^{-\alpha}$ nerves, we observed $27.27 \%$ (18 out of 66 nerves) with strong pSmad3 expression, 19.7\% (13 out of 66 nerves) with moderate pSmad3 expression, 16.67\% (11 out of 66 nerves) with low pSmad3 expression and 36.36\% (24 out of 66 nerves) with no pSmad3 expression (Figure 4-9C). Overall, for nerves that were positive for pSmad3 labeling, we observed a greater percentage of nerves with low expression in wildtype controls as compared to a greater percentage of nerves with strong expression in $e r b b 3 b^{-/-}$ larvae (Figure 4-9C). To more objectively quantify this observation, we measured the 
fluorescence intensity of $\mathrm{pSmad} 3$ labeling by outlining $\mathrm{pSmad}^{+}$regions along spinal motor nerves in 8 dpf wildtype (Figure 4-9A, bottom panels) and erbb3b $b^{-/}$(Figure 4-9B, bottom panels) larvae. After correcting for background fluorescence, we observed a statistically significant increase in the fluorescence intensity in $e r b b 3 b^{-/-}$larvae (average $=$ 66717 A.U., $n=66$ nerves) when compared to wildtype control (average $=35369$ A.U., $n$ $=66$ nerves) (Figure 4-9D). Additionally, to confirm that the ectopic OPCs were contributing to the increase in pSmad3 labeling we observed along erbb3b $b^{-/}$motor nerves, we compared pSmad3 labeling along wildtype and erbb3 $b^{-/}$nerves that were populated by myelinating glia. We observed a significantly larger proportion of nerves that were $\mathrm{pSmad}^{+}$in the mutants, with approximately $75 \%$ of $\operatorname{erbb3} b^{-/}$nerves $(\mathrm{n}=32$ nerves) containing ectopic OPCs that were $\mathrm{pSmad}^{+}$in comparison to wildtype in which only $25 \%$ of nerves $\left(n=37\right.$ nerves) were $\mathrm{pSmad}^{+}$(Figure $\left.4-9 \mathrm{E}\right)$. In the small number of $e^{2} b b 3 b^{-/}$nerves that did not have ectopic oligodendrocytes, we observed that approximately $60 \%$ of nerves did not have pSmad3 labeling (data not shown). These results demonstrate that ectopic OPCs are contributing to the increase in pSmad3 labeling observed in $e r b b 3 b^{-/-}$larvae.

Because nerves that were positive for $\mathrm{pSmad} 3$ expression in $8 \mathrm{dpf} e r b b 3 b^{-/-}$larvae had stronger labeling (Figure 4-9C - 4-9D), we hypothesized that using a selective inhibitor of the TGF- $\beta 1$ receptor (SB431542), previously used in Xenopus (Ho et al., 2006), zebrafish (Ho et al., 2006; Sun et al., 2006) and mouse (Seo et al., 2014), would inhibit TGF- $\beta$ signaling and decrease the extent of ZO-1 labeling more significantly along mutant nerves compared to wildtype. Since TGF- $\beta$ is important for early embryogenesis, 

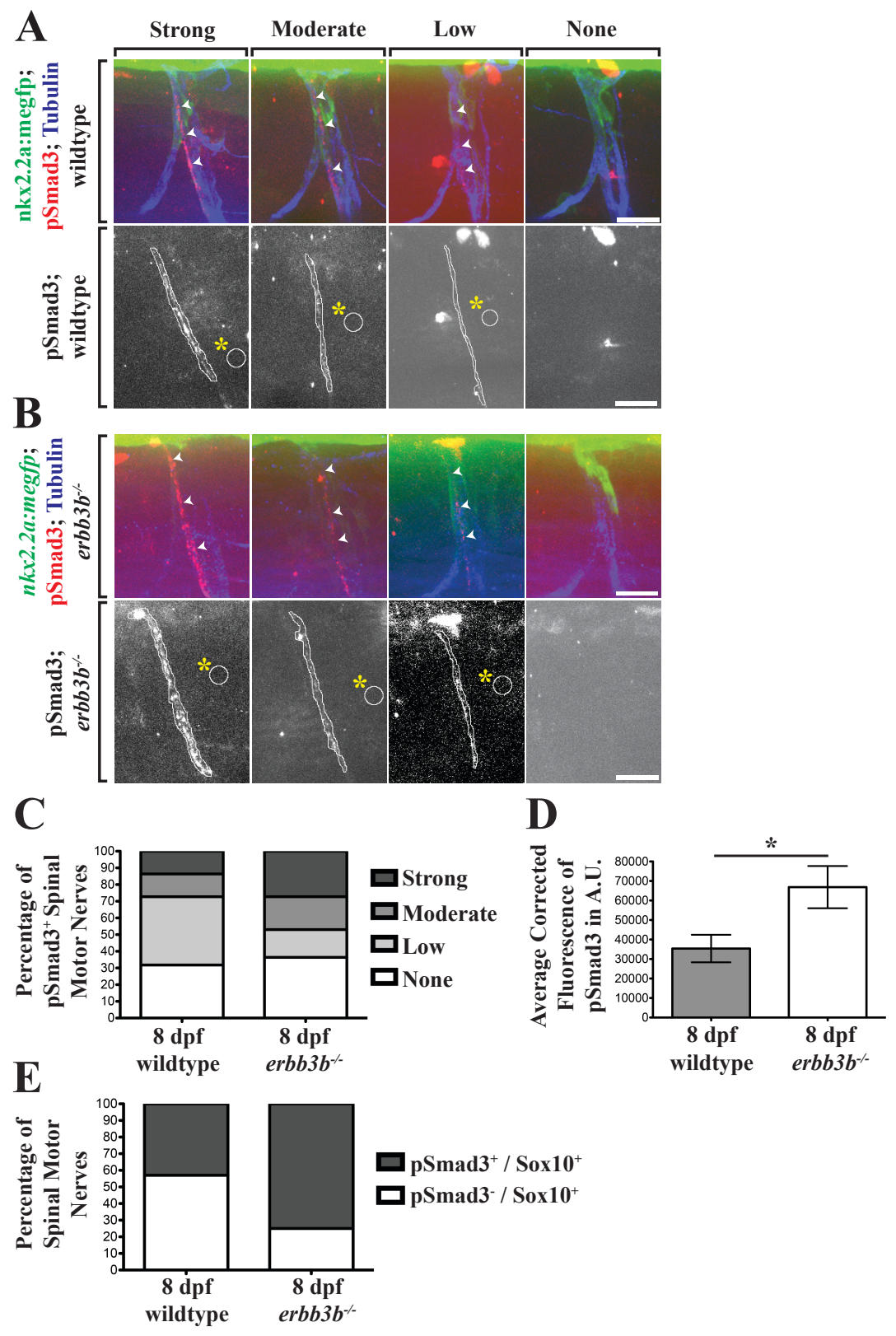

Figure 4-9. Phosphorylated Smad3 levels are increased along erbb3 $b^{-/}$larval spinal motor nerves. All images are lateral views of $8 \mathrm{dpf} n k x 2.2 a$ :megfp wildtype and $e r b b 3 b^{-/}$larvae, with dorsal to the top and anterior to the left, labeled with an antibody to $\mathrm{pSmad} 3$ (red) and tubulin (blue). (A\&B) At $8 \mathrm{dpf}$, pSmad3 labeling (red, arrowheads) along wildtype and erbb3 $b^{-/}$spinal motor axons can be classified into the following categories: Strong, Moderate, Low and None. The area used to quantify the average corrected fluorescence intensity of pSmad3 labeling is outlined in the bottom panels of A \& B and the asterisk denotes the area used for calculating the background fluorescence. (C) Quantification of pSmad3 labeling. (D) Average fluorescence intensity of pSmad3 in A.U., corrected for background fluorescence, in $8 \mathrm{dpf}$ wildtype and $e r b b 3 b^{-/}$larvae. There is significantly greater pSmad3 fluorescence intensity along erbb3 $3 b^{-\alpha}$ motor nerves as compared to wildtype controls $(\mathrm{p}=0.0158)$. Statistical significance was measured using an unpaired t-test. (E) Quantification of motor nerves with myelinating glia that are pSmad3 ${ }^{+}$versus pSmad3: Scale bars, $25 \mu \mathrm{m}$. 
we validated that SB431542 was effectively working by treating one-cell stage zebrafish embryos for approximately 24 hours with 100 or $200 \mu \mathrm{M}$ SB431542 dissolved in $0.5 \%$ DMSO or $0.5 \%$ DMSO alone. In inhibitor-treated embryos, we observed concentration dependent morphological changes in $24 \mathrm{hpf}$ embryos that were consistent with previously reported findings (data not shown) (Sun et al., 2006).

To validate that SB431542 was inhibiting TGF- $\beta 1$ at later stages, we treated 6 dpf wildtype zebrafish with $25 \mu \mathrm{M}$ SB431542 dissolved in $0.5 \%$ DMSO or with $0.5 \%$ DMSO alone for approximately 48 hours, fixed at $8 \mathrm{dpf}$ and performed immunohistochemistry with an antibody to pSmad3. In wildtype larvae exposed to $0.5 \%$ DMSO from 6 to $8 \mathrm{dpf}$, we observed pSmad3 labeling along approximately 53.03\% of motor nerves $(\mathrm{n}=66$ nerves) (Figure 4-10A). However, in $8 \mathrm{dpf}$ wildtype larvae treated with $25 \mu \mathrm{M}$ SB431542, only $30.91 \%$ of motor nerves $(n=55$ nerves) had pSmad3 labeling (Figure 410B), a significant decrease as compared to the DMSO control. Next, we categorized pSmad3 labeling along spinal motor nerves into strong, moderate, low and no expression for both the DMSO and SB431542 groups. In the DMSO group, approximately $12.12 \%$ ( 8 out of 66 nerves) had strong expression, 15.15\% (10 out of 66 nerves) had moderate expression, 25.76\% (17 out of 66 nerves) had low expression and 46.97\% (31 out of 66 nerves) had no pSmad3 expression (Figure 4-10C). In comparison, the SB431542 group had $1.82 \%$ ( 1 out of 55 nerves) with strong expression, $5.45 \%$ ( 3 out of 55 nerves) with moderate, $23.64 \%$ (13 out of 55 nerves) with low expression and $69.09 \%$ (38 out of 55 nerves) with no expression (Figure 4-10C). 
After validating that SB431542 was functional in our hands, we proceeded to treat $n k x 2.2 a: m e g f p$ wildtype and $e r b b 3 b^{-/}$larvae from 6 to 8 dpf with $0.5 \%$ DMSO or $25 \mu \mathrm{M}$ SB431542 dissolved in 0.5\% DMSO (Figure 4-10D - 4-10G). At 8 dpf, we investigated the morphology of the $n k x 2.2 a: m e g f p^{+}$perineurial glial cells and observed there were significantly more perineurial glia that appeared patchy / discontinuous in erbb3b $b^{-/}$ treated with $25 \mu \mathrm{M}$ SB431542 as compared to $e r b b 3 b^{-/-}$treated with $0.5 \%$ DMSO (data not shown). To investigate the effects that inhibiting TGF- $\beta 1$ had on $\mathrm{ZO}-1$ labeling, we treated larvae from 6 to $8 \mathrm{dpf}$, fixed the larvae at $8 \mathrm{dpf}$, performed immunohistochemistry using an antibody to ZO-1 and imaged all groups (Figure 4-10D - 4-10G). After imaging, we measured the extension length of ZO-1 labeling from the MEP TZ ventrally towards the horizontal myoseptum. In these studies, we observed a statistically significant reduction in the extension length of ZO-1 labeling in 8 dpf wildtype larvae treated with SB431542 (average $30.95, \mathrm{n}=140$ nerves) as compared to $8 \mathrm{dpf}$ larvae treated with DMSO (average 40.26, $\mathrm{n}=102$ nerves) (Figure 4-10H). Similarly, there was a statistically significant reduction in the extension length of ZO-1 labeling along spinal motor nerves in $8 \mathrm{dpf} e r b b 3 b^{-/}$larvae treated with SB431542 (average 24.72, $\mathrm{n}=108$ nerves) as compared to DMSO-treated $\mathrm{erbb3b}^{-/-}$(average 38.00, $\mathrm{n}=146$ nerves) (Figure 4-10H). Intriguingly, the extension length of $\mathrm{ZO}-1$ labeling in $8 \mathrm{dpf} e r b b 3 b^{-/-}$treated with SB431542 (average $24.72, \mathrm{n}=108$ nerves) was significantly decreased as compared to 8 dpf wildtype treated with SB431542 (average 30.95, $\mathrm{n}=140$ nerves) (Figure 4-10H). Taken together, these results demonstrate that pSmad3 expression is stronger along erbb3 $3 b^{-/}$motor nerves and inhibiting TGF- $\beta 1$ signaling in these mutants has a 

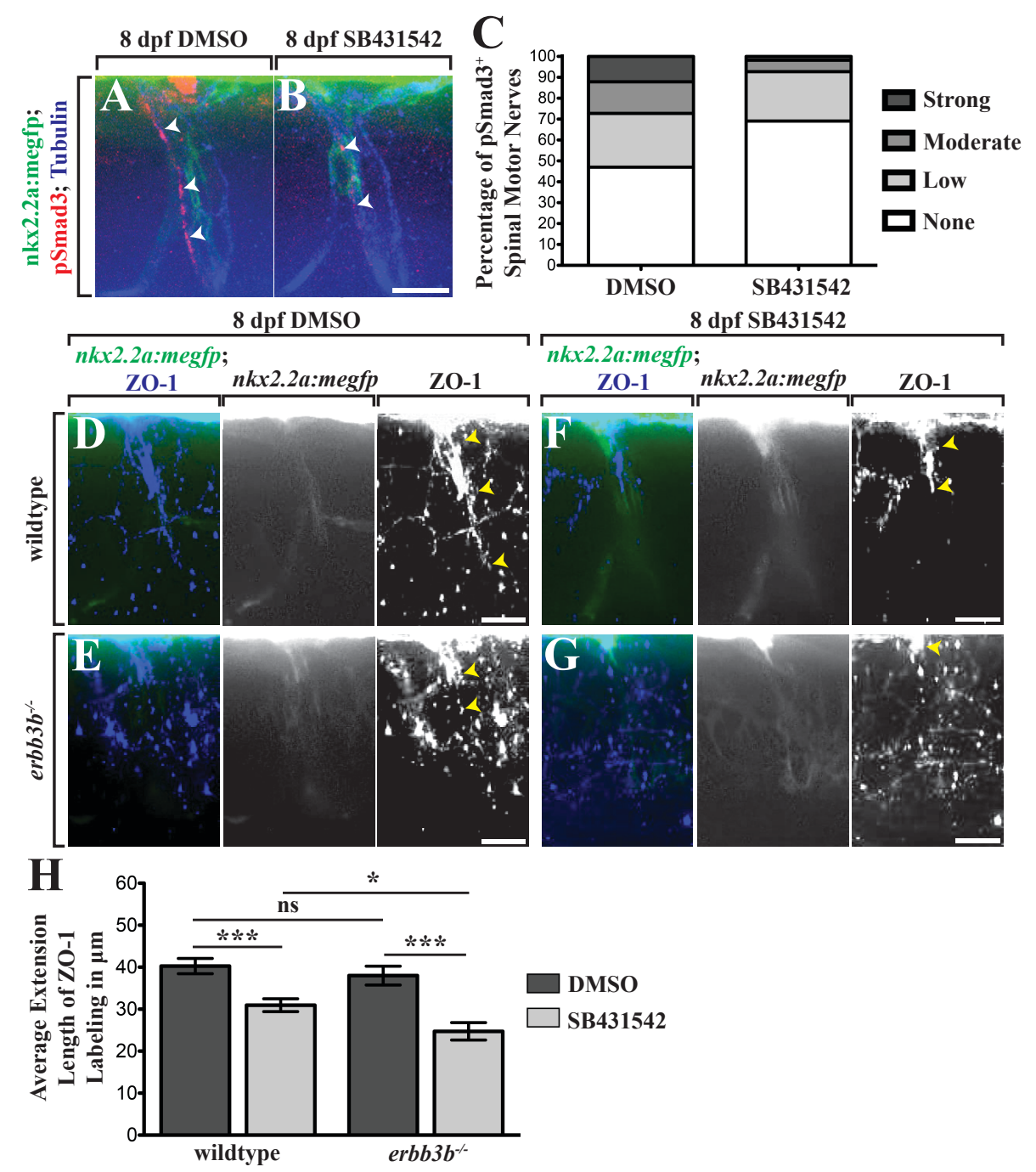

Figure 4-10. Inhibiting TGF- $\beta 1$ decreases ZO-1 labeling along spinal motor nerves. All images are lateral views of $8 \mathrm{dpf} n k x 2.2 a$ :megfp wildtype and $e r b b 3 b^{-/}$larvae, with dorsal to the top and anterior to the left. (A) pSmad3 labeling (arrowheads) is evident along motor nerves in 8 dpf $n k x 2.2 a$ :megfp wildtype larvae treated with $0.5 \%$ DMSO from 6 to $8 \mathrm{dpf}$. (B) pSmad3 labeling (arrowheads) was reduced along motor nerves treated with $25 \mu \mathrm{M}$ SB431542 from 6 to $8 \mathrm{dpf}$. (C) Quantification of pSmad3 labeling in $8 \mathrm{dpf}$ nkx2.2a:megfp wildtype larvae treated with $0.5 \%$ DMSO or $25 \mu \mathrm{M}$ SB431542 between 6 and 8 dpf. (D-E) At 8 dpf, $n k x 2.2 a$ :megfp wildtype (D) and $e r b b 3 b^{-\circ}$ (E) larvae exposed to $0.5 \%$ DMSO from 6 to 8 dpf have ZO-1 labeling (arrowhead) from the MEP TZ and it extends ventrally towards the horizontal myoseptum. (F-G) When treated with $25 \mu \mathrm{M}$ SB431542, ZO-1 labeling (arrowhead) is reduced in $8 \mathrm{dpf}$ $n k x 2.2 a$ :megfp wildtype (F) and erbb3b $b^{-/}(\mathrm{G})$ larvae. (H) Quantification of the extension length of ZO-1 labeling from panels D-G. There is a statistically significant reduction in the extension length of ZO-1 labeling along spinal motor nerves in 8 dpf wildtype larvae treated with $25 \mu \mathrm{M}$ SB431542 as compared to wildtype larvae treated with $0.5 \%$ DMSO $(\mathrm{p}=0.0001)$. Similarly, $8 \mathrm{dpf}$ erbb3b $b^{-/}$larvae treated with $25 \mu \mathrm{M}$ SB431542 had a significant reduction in ZO-1 labeling as compared to erbb3 $3 b^{-/}$larvae treated with $0.5 \%$ DMSO ( $\mathrm{p}<0.0001)$. Intriguingly, there is also a statistically significant reduction in the extension length of ZO-1 labeling along spinal motor nerves in $8 \mathrm{dpf}$ erbb3b larvae treated with $25 \mu \mathrm{M}$ SB431542 as compared to wildtype larvae treated with $25 \mu \mathrm{M}$ SB431542 ( $\mathrm{p}=0.0144)$. Statistical significance was measured using an unpaired t-test. Scale bars, $25 \mu \mathrm{m}$. 
statistically significant effect on perineurial glial maturation as compared to TGF- $\beta 1$ inhibition in wildtype controls.

\section{Discussion}

Spinal motor nerves are important conduits for relaying information from the CNS to the PNS. Forming a functional motor nerve requires precise interactions between various cell populations, including glial cells. Previously, we have shown that Schwann cells require perineurial glia for migration, differentiation and myelination (Binari et al., 2013; Kucenas et al., 2008b) and, reciprocally, perineurial glia require Schwann cells for proper development and response to injury (Kucenas et al., 2009; Kucenas et al., 2008b; Lewis \& Kucenas, 2014; Parmantier et al., 1999; Sharghi-Namini et al., 2006). These studies demonstrate that peripheral glial-glial interactions are essential during motor nerve assembly, but what happens in disease states in which myelin sheath perturbations occur? Are the remaining ensheathing glial cells, specifically perineurial glia, able to compensate for this aberration and maintain a functional nerve?

Here, we provide evidence that, despite the presence of centrally-derived myelin along erbb3b mutant motor nerves, perineurial glia are capable of exiting the CNS, extending along peripheral motor nerves during development and expressing $\mathrm{ZO}-1$, a marker of tight junctions and perineurial glial maturation. Our ultrastructural data corroborates our in vivo imaging and immunohistochemistry findings, i.e., the perineurium is present in $e r b b 3 b^{-/}$adults. However, in some areas, perineurial morphology is comparable to wdiltype whereas other areas are disorganized or patchy (Figure 4-4E, right panel) and 
we observed large pockets of high levels of tight junctions (Figure 4-7H, right panel). Additionally, the perineurium barrier function is compromised in $e r b b 3^{-/}$zebrafish as we observed a fluorescent dye infiltrate within the motor nerve (Figure 4-8B), and we hypothesize that the leaky barrier may be due to the observed morphological alterations described above. Future studies further characterizing the development of the perineurial barrier will help provide a better understanding about mechanisms underlying how it is formed and ultimately functions. However, despite the complete absence of peripheral myelinating glial cells, perineurial glia are remarkably plastic in response to injury by forming phagocytic vesicles and glial bridges spanning the injury site. Although the presence of OPCs from the spinal cord does not have detrimental effects on certain perineurial glial developmental and injury response behaviors, the barrier integrity is ultimately impaired. Therefore, normal peripheral glial-glial interactions are important for development and maintenance of spinal motor nerves (Parmantier et al., 1999; Sharghi-Namini et al., 2006).

\section{Is erbb3b an important mechanism for mediating perineurial glial development and maturation?}

The molecular mechanisms involved in perineurial glial development and maturation are poorly understood. Desert hedgehog (Dhh), derived from Schwann cells, is important for perineurium maturation as $D h h^{-/}$mice have a patchy, disorganized perineurium with abnormal tight junction formation (Parmantier et al., 1999). However, it is unknown when, and if, hedgehog signaling is present along the peripheral nerves in $e r b b 3 b^{-/}$ 
mutants since neural crest-derived Schwann cells fail to associate with motor nerves (Figure 4-2B) (Smith et al., 2014).

In this study, we used a zebrafish line with a mutation in the erbb3b receptor tyrosine kinase as a model for perturbing peripheral myelinating glial cells (Lyons et al., 2005), leading to the ectopic migration of OPCs (Smith et al., 2014), to investigate if perineurial glia are affected by the presence of centrally-derived myelin. However, it is unknown if perineurial glial cells express erbb3b, and if so, if it is the perturbation in erbb3b, or the presence of ectopic OPCs and lack of peripheral myelinating glia, that is responsible for our results. To investigate this, we used a zebrafish line with a mutation in neuregulin I type III ( $\left.\mathrm{rrg}^{226--}\right)$ (Perlin et al., 2011), an axonally-expressed growth factor that binds to the erbb2-erbb3 heterodimer receptor complex on peripheral myelinating glial cells to initiate the peripheral myelination signaling cascade (Brinkmann et al., 2008; Chen et al., 2006). In $\mathrm{nrg}^{-/-}$larvae, peripheral myelinating glia fail to associate with spinal motor nerves and OPCs ectopically exit the CNS (data not shown), but erbb3b is intact. Intriguingly, we observed perineurial glial migration, proliferation and extension behaviors in $\mathrm{nrg}^{-/-}$larvae (data not shown) that are similar to those we observed in $e r b b 3 b^{-/}$larvae. Although future studies are needed to definitively rule out if perineurial glia express erbb3b, the results we observed in $n r^{-/-}$larvae are consistent with the hypothesis that loss of erbb3b does not directly affect perineurial glia and, instead, centrally-derived myelinating glial cells are what influence perineurial glial developmental and regenerative behaviors in $\operatorname{erbb} 3 b^{-/}$larvae. 


\section{Do ectopically-located OPCs impair perineurial glial injury responses?}

Peripheral nerves demonstrate remarkable regenerative capabilities, a phenomenon that the CNS lacks (Faroni et al., 2015). Following PNS axonal transection, Schwann cells transdifferentiate to help with regenerative processes and, as we have recently shown, perineurial glia are essential components also involved in injury responses (Lewis \& Kucenas, 2013; 2014). In this study, we demonstrate that transecting a nerve containing ectopically-located OPCs impairs perineurial membrane extension towards an injury site. However, perineurial-perineurial injury response behaviors, such as forming phagocytic vesicles and a glial bridge spanning the transection site, are not impaired on axonal tracts that do not contain ectopically-located OPCs. This raises the intriguing possibility that OPCs have remarkable control over regeneration, and that it is not necessarily the permissive environment of the PNS, but rather the permissive qualities of the peripheral ensheathing glial cells themselves, that are responsible for the remarkable repair that typically occurs in the PNS. Since it has previously been reported that oligodendrocytes may impede CNS regeneration by expressing axonal growth inhibitors (Busch \& Silver, 2007; Caroni \& Schwab, 1988; Faroni et al., 2015; Fawcett, 2006), the lack of peripheral myelinating glial cells, combined with the presence of OPCs along spinal motor nerves, may be a combination that impairs certain perineurial glial regenerative behaviors in $e r b b 3 b^{-/}$larvae that are required for PNS repair. Future studies investigating if OPCs located along $e r b b 3 b^{-/}$nerves secrete or express signaling cues that negatively affect perineurial cells are exciting next steps for understanding the regenerative potential of both the CNS and PNS. 
Although mechanisms involved in perineurial glial regeneration behaviors are largely unknown, one potential mechanism that may be involved is TGF- $\beta 1$. A previous study demonstrated that applying a neutralizing antibody to TGF- $\beta 1$ via perineurial administration after axonal transection improved sciatic nerve regeneration (Davison et al., 1999). This report, in combination with our observation of phosphorylated Smad3, a downstream signaling transducer of TGF- $\beta 1$, along spinal motor nerves, leads us to hypothesize that inhibiting TGF- $\beta 1$ may enhance perineurial glial injury responses. Future studies aimed at investigating the role of TGF- $\beta 1$ as well as identifying additional signaling cues that mediate the various perineurial injury responses may reveal novel therapeutic targets for treating peripheral nerve injuries and promoting regeneration.

\section{Do ectopic OPCs increase zonula occludens-1 labeling within the blood-nerve barrier?}

Above we show that perineurial glia have an increase in the extension of ZO-1 labeling along $e r b b 3 b^{-/}$motor nerves as compared to wildtype controls, leading us to hypothesize that the presence of OPCs along spinal motor nerves may be influencing this increase by helping to promote the integrity of the blood-nerve barrier. A recent study demonstrated that OPCs help support and promote the integrity of the blood-brain barrier, as evidenced by an increase in ZO-1 labeling and the formation of tight junctions by endothelial cells, both in vitro and in vivo (Seo et al., 2014). OPCs secrete transforming growth factor $\beta 1$ (TGF- $\beta 1$ ) in the CNS, activating the MEK/ERK signaling cascade, eventually leading to tight junction protein expression by endothelial cells of the BBB (Seo et al., 2014). We

investigated the involvement of TGF- $\beta 1$ on ZO- 1 expression in the blood-nerve barrier 
by inhibiting TGF- $\beta 1$ signaling in both wildtype \& mutant larvae. We observed a significant decrease in the extension of ZO-1 labeling in erbb3 $3 b^{-/}$larvae as compared to wildtype. This result demonstrates that OPCs are partially responsible for the increase in extension of ZO-1 labeling observed in erbb3 $3 b^{-/}$. Additionally, we observed a decrease in the extent of ZO-1 labeling along wildtype motor nerves treated with the TGF- $\beta 1$ inhibitor as well, although the reduction was not as great as in erbb $3 b^{-/}$larvae. This suggests that TGF- $\beta 1$ is involved in wildtype perineurial glial development as well. Schwann cells are known to express TGF- $\beta 1$ during development (Feng \& Ko, 2008; Scherer et al., 1993) and because of this, may be a likely source of this ligand for perineurial glia.

Taken together, our work reinforces the importance of glial-glial interactions along spinal motor nerves during development and in response to injury. Intriguingly, our data reveal that perineurial glia can adapt to the presence of ectopically-located, centrally-derived OPCs for certain developmental behaviors and injury responses, demonstrating their incredible plasticity in response to myelinopathies and axonal transections. Furthermore, we identified that TGF- $\beta 1$ has an influence on ZO-1 expression, a marker of perineurial differentiation (Kristensson \& Olsson, 1971; Pummi et al., 2004). Although perineurial glial cells demonstrate remarkable plasticity to myelin perturbations, their barrier function is compromised, resulting in a nerve that is left unprotected and vulnerable. Therefore, our data further support the importance of Schwann cell-perineurial glial interactions in forming and maintaining functional peripheral nerves (Parmantier et al., 1999; Sharghi-Namini et al., 2006). 
Acknowledgements: We would like to thank members of the Kucenas laboratory for valuable discussions and Lori Tocke for zebrafish care. For the transmission electron microscopy experiments, we would especially like to thank the following: Dr. Alev Erisir and Erisir laboratory members, Bonnie Sheppard and Drs. Stacey Criswell and Yalin Wang from the UVA Advanced Microscopy Facility. This work was supported by the National Institutes of Health (NIH): NS072212 (SK) and NS051140 (GML), the March of Dimes: 5-FY11-90 (SK) and the Hartwell Foundation (SK). 


\title{
Chapter 5
}

\section{Visualizing demyelination in vivo in a novel drug-induced demyelination model in zebrafish}

\begin{abstract}
In multiple sclerosis (MS), the immune system inappropriately attacks the insulating myelin sheaths surrounding central nervous system (CNS) axons. Unfortunately, the initial trigger leading to this inappropriate attack on myelin is not well understood. Although mammalian models and clinical patient samples are commonly used to study MS, these models do not allow for direct visualization of this disease in vivo. To overcome this limitation, we created and characterized a novel, focal drug-induced demyelination model in zebrafish that allows us to investigate temporal dynamics underlying myelin breakdown and repair in vivo. We injected lysolecithin, a demyelinating drug, into 4 to 6 day post fertilization (dpf) zebrafish spinal cords and, coupled with in vivo, time-lapse imaging, observed hallmarks consistent with other models of demyelination, including myelinating glial loss, myelin perturbations, axonal sparing and debris clearance events. Thus, we have developed and characterized a novel demyelination model in zebrafish that allows us to visualize myelin breakdown and repair in a living, vertebrate organism. This model will be a useful pre-clinical screening tool for investigating the safety and efficacy of novel therapeutic compounds to treat demyelinating disorders like MS.
\end{abstract}




\section{Introduction}

Multiple sclerosis (MS) is considered a neuroinflammatory disorder characterized by the inappropriate attack of the myelin sheath by the immune system, which results in demyelination, axonal degeneration and eventually, severe disability. MS primarily attacks the central nervous system (CNS) in the form of focal demyelinating lesions found within the brain, spinal cord and/or optic nerve, and patients battle various neurological challenges that are unique to lesion location. A debilitating component of the disease is its relapsing-remitting nature, making it impossible to predict when, or for how long, a relapse will occur. Unfortunately, disease chronicity often results in an increase in symptom severity, with patients experiencing longer relapses and fewer remission periods, eventually transitioning into a secondary-progressive phase (Confavreux et al., 2000; Hartman \& Rosenzweig, 2010; Pozzilli et al., 2010).

Although disease modifying therapeutic strategies like interferon beta, natalizumab, fingolimod, teriflunomide and dimethyl fumarate help reduce relapse severity and slow disease progression, MS patients often progress to severe disability and rely on walking aids and assistance (Carvalho \& Sá, 2012; Gajofatto \& Benedetti, 2015; Hartman \& Rosenzweig, 2010; Pozzilli et al., 2010). Unfortunately, what causes MS, why the immune system attacks myelin or how demyelination occurs, remains unknown. Because MS is such a heterogeneous disease, with diversity found in clinical symptoms, pathology subtypes, therapy response and radiology findings (Denic et al., 2011), the unknown etiology of MS has led to two main hypotheses known as the outside-in model and inside-out model (Stys et al., 2012). The outside-in hypothesis suggests that the immune 
system is initially triggered and becomes primed to infiltrate into the CNS to attack myelin sheaths (McFarland \& Martin, 2007; Stys et al., 2012). In contrast, the inside-out hypothesis suggests that the initial, or primary, insult is cytodegeneration of the oligodendrocyte and myelin that subsequently triggers an immune response (Barnett \& Prineas, 2004; Henderson et al., 2009; Stys et al., 2012; Traka et al., 2015). The heterogeneity of patient lesions, classified as Pattern I - Pattern IV, further supports the difficulties in identifying the etiology, as Patterns I and II demonstrate T-cell inflammatory mediated demyelination and Patterns III and IV suggest oligodendrocyte and myelin cytodegeneration (Denic et al., 2011). Recently, an elegant study in mice demonstrated that oligodendrocyte death primed the immune system to attack myelin at a later time point, weeks after the mice had recovered from the initial insult (Traka et al., 2015). However, our understanding of the processes underlying oligodendrogliopathy is limited, but, as this may be the initial trigger or hit for some MS patients, studies aimed at further investigating this mechanism for disease initiation are important.

Mammalian models commonly used to study MS, including experimental autoimmune encephalomyelitis (EAE) and drug-induced demyelination, as well as patient data or clinical samples, do not allow researchers to directly visualize processes underlying myelin destruction and repair in vivo. Although a generalized demyelination model exists in zebrafish that takes advantage of the ease of drug submersion combined with tissuespecific ablation by the bacterial nitroreductase gene, a major limitation of this model is the inability to create focal lesions, which are a hallmark of MS pathology (Chung et al., 2013; Fang et al., 2015). To overcome this limitation, we have created and characterized 
a model in zebrafish that induces focal demyelinating lesions by injecting lysolecithin into the spinal cord, thus, allowing us to visualize myelin destruction and repair in a living, intact vertebrate model system.

\section{Results}

\section{Creating a focal demyelination model in zebrafish}

Lysophosphatidylcholine, commonly referred to as lysolecithin, has been used in mammalian models, including mice, rats, rabbits, cats and Macaque monkeys, to induce demyelinating lesions in the spinal cord and the corpus callosum (Blakemore et al., 1977; Blakemore \& Franklin, 2008; Dousset et al., 1995; Foote \& Blakemore, 2005; Girard et al., 2005; Gregg et al., 2007). Injections of $1 \%$ lysolecithin is sufficient to induce demyelination in the CNS of these animals and evidence of remyelination begins between 7 and 10 days post injection (dpi) (Jeffery \& Blakemore, 1995; Kotter et al., 2001). Although $1 \%$ lysolecithin placed on gelatin foam can successfully induce demyelination when applied directly to the adult zebrafish optic nerve, neither the effects of lysolecithin injections in the zebrafish spinal cord or the in vivo visualization of de- or re-myelination have been described (Münzel et al., 2014). Therefore, we sought to create a focal druginduced demyelination model in zebrafish by injecting into the spinal cord of 4 to $6 \mathrm{dpf}$ larvae (Figure 5-1A).

Prior to injecting, we mounted larvae laterally on $2 \%$ agar pads solidified onto a $22 \mathrm{~mm} \mathrm{x}$ $60 \mathrm{~mm}$ borosilicate glass coverslip (Figure 5-1B). The coverslip containing the mounted larvae was placed directly onto a Zeiss Axio Observer Z1 microscope stage equipped 
with an ASI MPPI-3 pressure injection rig and mechanical micromanipulator (Figure 51C). A micropipette needle containing either a $1 \mathrm{X}$ phosphate buffered saline (PBS) control or $0.875 \%$ lysolecithin experimental solution in $1 \mathrm{X}$ PBS was placed in to the dorsal spinal cord. When the micropipette was not inserted successfully into the spinal cord, the solution was inappropriately injected into the muscle, evidenced by the muscle appearing wavy upon solution dispersal (Figure 5-1D). Successful insertions into the spinal cord resulted in the solution remaining localized within the spinal cord (Figure 51E). To confirm that injection volumes were comparable between control and experimental groups, we included a fluorescent dye (Dextran-647) with the injection cocktail. In these studies, we observed comparable dispersal between the control (Figure 5-1F) and experimental solutions (Figure 5-1G), approximately two somites anterior and posterior to the injection site. Furthermore, we also observed a comparable survival rate post-injection for both groups, with the control survival at $72.67 \%(\mathrm{n}=236)$ and experimental survival at $69.4 \%(n=183)$ (data not shown). From these studies, we present a method to dispense solutions focally and precisely into the zebrafish larval spinal cord with a high survival rate.

\section{Lysolecithin alters the number of $\operatorname{Sox}^{+}$cells within the lesion area}

Myelination in the zebrafish CNS occurs in an anterior to posterior manner, commencing around 3 dpf (Almeida et al., 2011; Czopka et al., 2013). Therefore, the presence of maturing myelin sheaths between 4 to $6 \mathrm{dpf}$, coupled with the transparency of transgenic larvae, is ideal for the in vivo imaging of oligodendrocyte response and behavior following exposure to a demyelinating agent like lysolecithin. Previous publications have 

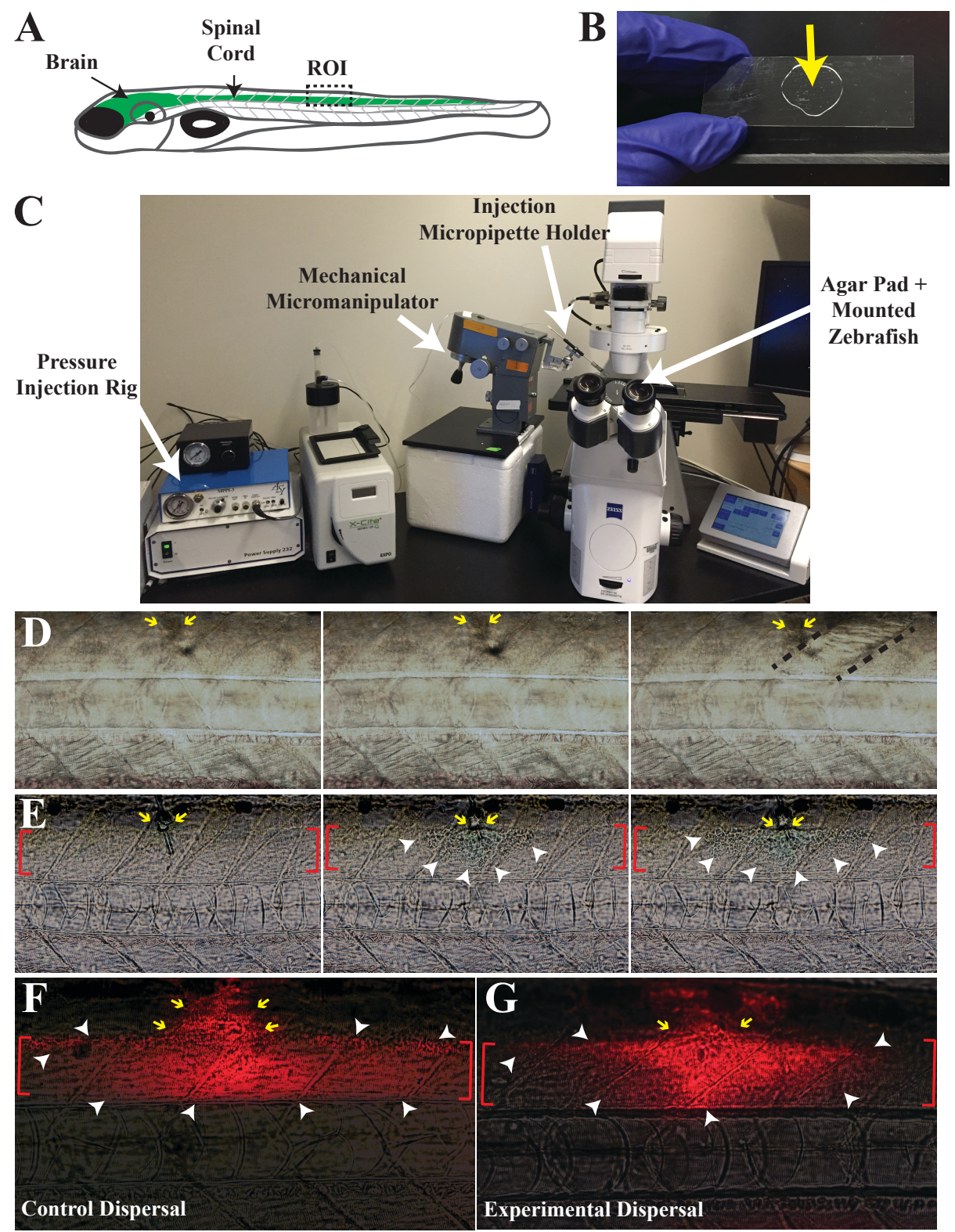

Figure 5-1. Focal delivery of solutions into the zebrafish spinal cord. (A) Cartoon of a zebrafish larvae identifying the brain and spinal cord (green). Boxed region identifies the injection site / region of interest (ROI). (B) Agar pad (arrow) solidified on a glass cover slip for mounting larvae. (C) Injection equipment set-up. (D) Muscle injections have a wavy appearance following dispersal. Yellow arrows: injection site; dotted line: muscle segment receiving solution. (E) Solutions remain localized within spinal cord following a successful injection. Red brackets: spinal cord; yellow arrows: injection site. white arrowheads: solution dispersal. (F - G) Dispersal region of water control (F) or lysolecithin solution $(\mathrm{G})$ containing the fluorescent tracer Dextran-647. Red brackets: spinal cord; yellow arrows: injection site; white arrowheads: dispersal region. 
reported a decrease in oligodendrocyte number, demyelination, OPC proliferation and OPC migration to the focal injury after injection of lysolecithin into the mammalian spinal cord (Azin et al., 2015; Blakemore et al., 1977; Blakemore \& Franklin, 2008; Keough et al., 2015; Kotter et al., 2001; Lau et al., 2012; Münzel et al., 2014).

To quantify the effects on oligodendrocyte number in our model, we inserted the micropipette needle into the spinal cord of 4 dpf sox 10:mrfp larvae at approximately somite 16 to 18 and injected either the lysolecithin cocktail $(0.875 \%$ lysolecithin $+0.21 \%$ Dextran 647) or a control solution (PBS $+0.21 \%$ Dextran 647). Following injection, we fixed the larvae at various time points post-injection and performed whole mount immunohistochemistry (IHC) with a Sox 10 antibody to count the number of Sox $10^{+}$cells in the spinal cord. We quantified the number of Sox $10^{+}$cells anterior to the injection site within a region of the spinal cord spanning approximately three motor nerves in width, to avoid effects caused by the injection injury itself and to capture the effects from either the drug or control solution (Figure 5-2A - 5-2E). The region posterior to the injection site was not quantified to reduce the risk of capturing a decrease in the number of oligodendrocytes that might be secondary to Wallerian degeneration (Waller, 1850). By 8 hours post injection (hpi), we observed a significant reduction in the number of Sox $10^{+}$ cells (Figure 5-2E) in the lysolecithin-injected group (Figure 5-2B) (average 24.94, $\mathrm{n}=6$ ) as compared to control larvae (Figure 5-2A) (average 30.33, $\mathrm{n}=5$ ), demonstrating lysolecithin is toxic to zebrafish oligodendrocytes. Intriguingly, we saw an increase in Sox $10^{+}$cells by 20 hpi in the lysolecithin group (Figure 5-2D) (average 32.96, $\mathrm{n}=8$ ) as compared to control (Figure 5-2C) (average 26.8, $\mathrm{n}=5$ ), which might mean that OPCs 


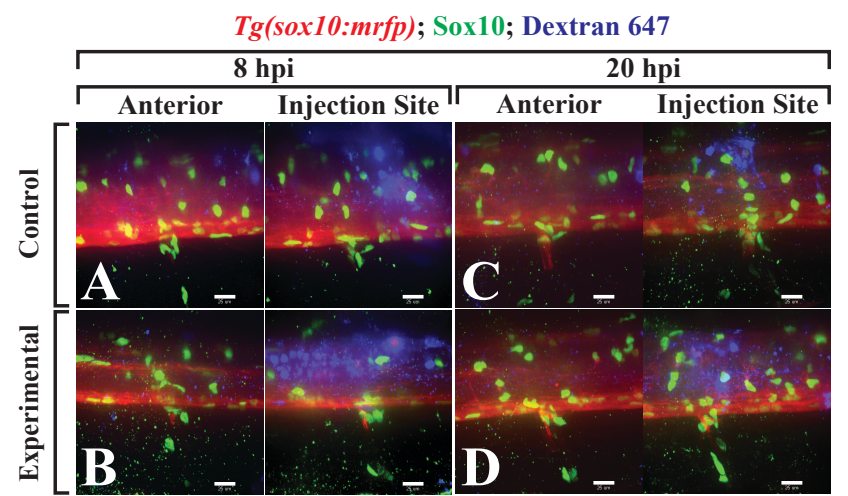

$\mathbf{E}$
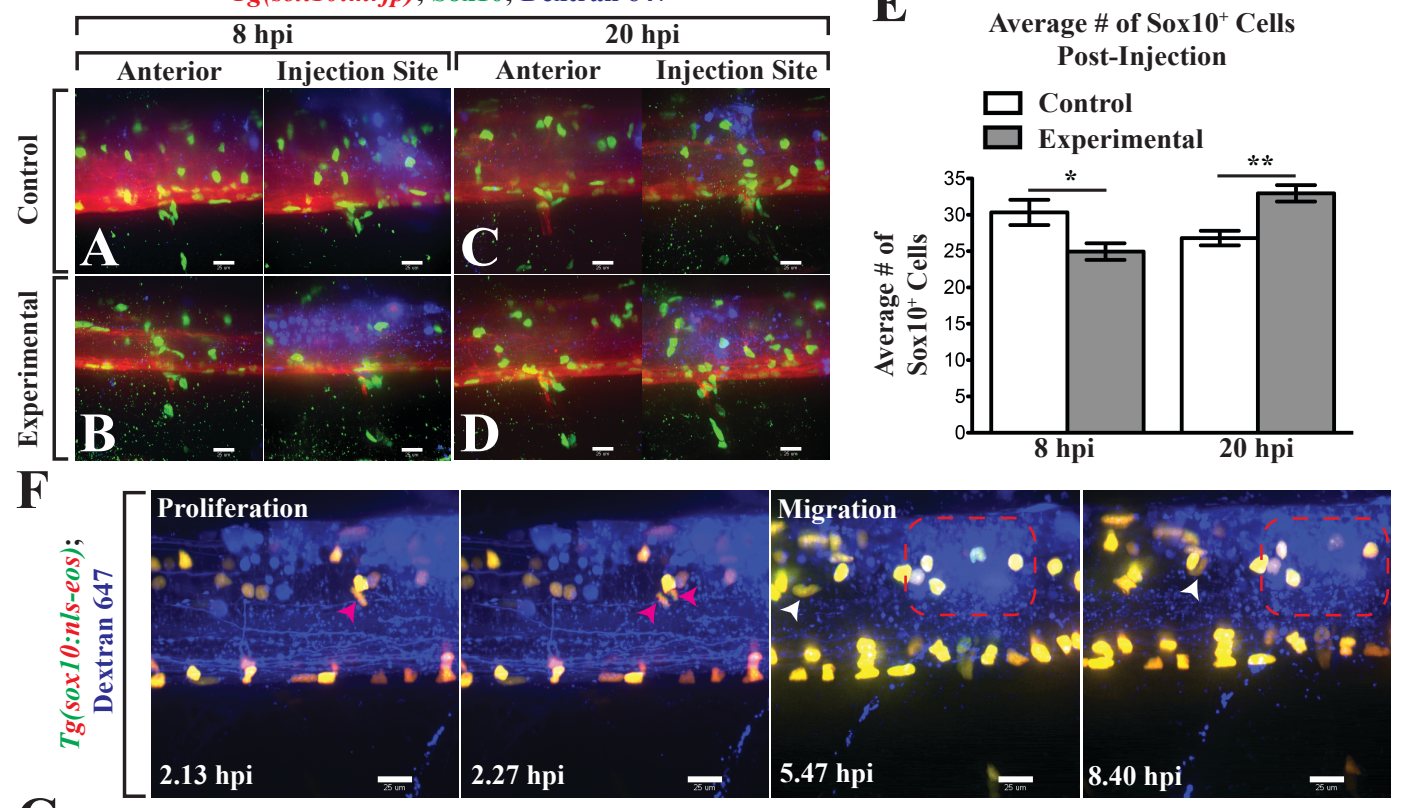

G

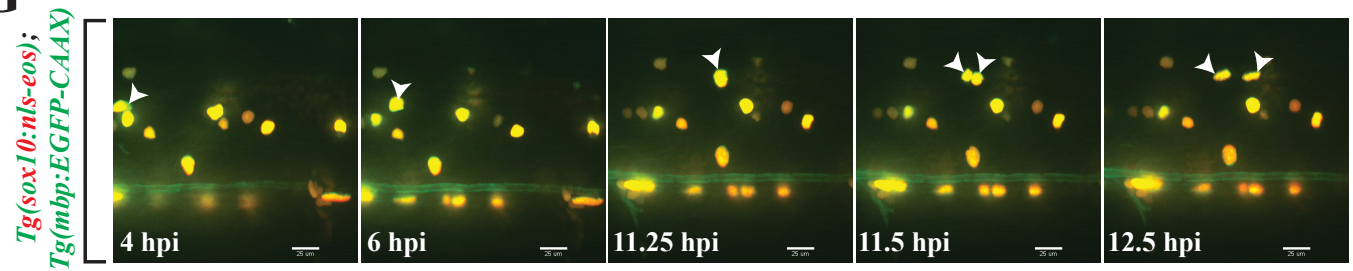

H

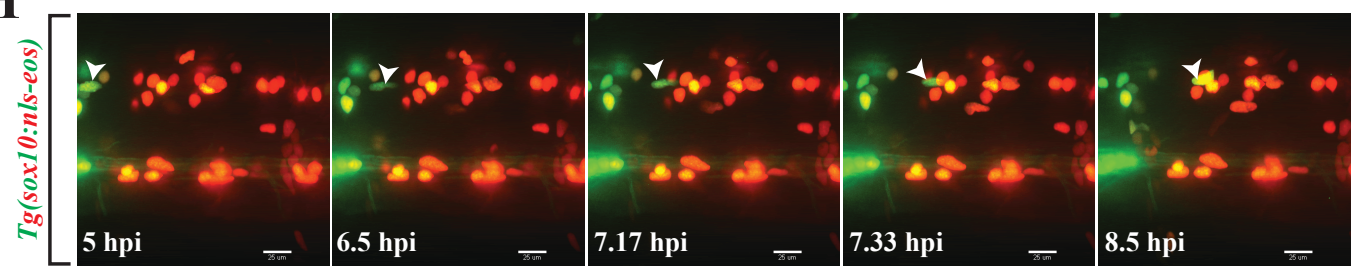

Figure 5-2. Lysolecithin effects on Sox10 ${ }^{+}$cells. All images are lateral views of spinal cord with anterior to left and dorsal to top. (A - D) 4 dpf sox 10:mrfp zebrafish larvae injected with control (2A, 2C) or lysolecithin (2B, 2D) containing Dextran-647. Larvae were fixed at 8 hpi (2A-2B) or 20 hpi (2C - 2D), labeled with a Sox 10 antibody (green cells) and Sox $10^{+}$cells anterior to injection site, but within dispersal region, were quantified. (E) There is a significant decrease in the average number of Sox $10^{+}$cells within the region anterior to injection site in the experimental (lysolecithin) group as compared to control at $8 \mathrm{hpi}\left({ }^{*} \mathrm{p}=0.0252\right.$ ), followed by a significant increase in the experimental (lysolecithin) group as compared to control by $20 \mathrm{hpi}$ $(* * \mathrm{p}=0.0033)$. Statistical significance was measured using an unpaired t-test. (F) Time-lapse imaging captures proliferation (pink arrowhead) and migration (white arrowhead) by two separate sox10:nls-eos ${ }^{+}$ cells following injection of lysolecithin into a $4 \mathrm{dpf}$ zebrafish spinal cord. Red dotted ellipse: injection site. (G) Time-lapse imaging captures proliferation and migration by an individual sox10:nls-eos ${ }^{+}$cell (arrowhead) following injection of lysolecithin into a 4 dpf zebrafish spinal cord. (H) Time-lapse movie following injection of lysolecithin and photoconversion of the lesion area in a 4 dpf sox10:nls-eos zebrafish larva. Red fluorescence denotes photoconverted cells within the lesion. Green fluorescence denotes cells outside of the lesion. Arrowhead identifies a sox10:nls-eos ${ }^{+}$cell anterior to injection site migrate posteriorly into the drug dispersal region. Scale bars, $25 \mu \mathrm{m}$. 
are proliferating in an attempt to repair the focal lesion, a phenomena reported in mammalian lysolecithin-induced demyelination models (Keough et al., 2015; NaitOumesmar et al., 1999; Sahel et al., 2015).

To better visualize OPC proliferation and migration in vivo, we injected the lysolecithin cocktail into 4 to 6 dpf $\operatorname{Tg}$ (sox10:nls-eos) (McGraw et al., 2012; Prendergast et al., 2012) larvae, in which sox $10^{+}$cells express nuclear-localized Eos. Following injection of lysolecithin, we observed two major OPC behaviors: 1) OPC migration and division from two separate $\operatorname{sox} 10^{+}$cells (Figure 5-2F) and 2) sox $10^{+}$OPC migration and division from a single sox $10^{+}$cell (Figure 5-2G). To investigate if $\operatorname{sox} 10^{+}$cells also migrated from areas anterior to the lesion, we used $\operatorname{Tg}(\operatorname{sox} 10: n l s-e o s)$ embryos to fate map OPCs. Following injection of lysolecithin, we photoconverted the Eos protein within the lesion, changing the green fluorescence to red via UV-light photoconversion (Prendergast et al., 2012; Smith et al., 2014; Stys et al., 2012; Wiedenmann et al., 2004). Next, we performed in vivo, time-lapse imaging and observed sox $10^{+}$photoconverted cells (red) within the lesion (Figure 5-2H) and $\operatorname{sox} 10^{+}$cells anterior to the dispersal region (green, unconverted). At approximately $6.5 \mathrm{hpi}$, a green $\operatorname{sox} 10^{+}$cell anterior to the lesion migrated posteriorly, and continued migration into the lesion (Figure 5-2H). This preliminary result demonstrates that a subset of $\operatorname{sox} 10^{+}$cells recruited to the lesion originate from areas anterior to the drug dispersal region. Interestingly, in our studies, we primarily observed dorsal OPCs proliferate to specify new OPCs, and we rarely captured ventral OPCs migrate towards the lesion area (data not shown). Taken together, these 
results confirm that lysolecithin affects the number and behavior of oligodendrocytes and their progenitors in the zebrafish spinal cord.

\section{Lysolecithin induces myelin membrane changes in zebrafish larvae}

Because we observed OPC loss, proliferation and recruitment in lysolecithin-injected larvae, we next sought to investigate whether lysolecithin induced myelin loss by performing in vivo, time-lapse imaging experiments in mbp:EGFP-CAAX (Almeida et al., 2011) larvae, where myelin is labeled with membrane-tethered GFP. Myelin basic protein is expressed in anterior regions of the zebrafish CNS beginning around $60 \mathrm{hpf}$ (Almeida et al., 2011). At later stages, between 4 and 6 dpf, we observed $\mathrm{GFP}^{+}$sheath-like structures in both the ventral and dorsal spinal cord (Czopka \& Lyons, 2011; Kearns et al., 2015) (Figure 5-3A - 5-3B). Following injection of a control solution into a $4 \mathrm{dpf}$ zebrafish spinal cord, the $\mathrm{GFP}^{+}$myelin sheaths remained unchanged throughout the duration of the time-lapse (Figure 5-3C). However, following injection of a lysolecithin solution, we observed robust membrane changes within the drug dispersal region. Specifically, we observed $\mathrm{GFP}^{+}$myelin sheaths change to ovoid-like structures, beginning around 2.5 hpi (Figure 5-3D). The formation of myelin ovoid-like / onion bulb structures has previously been reported as evidence of myelin degradation (Acar et al., 2004), and we did not observe the same phenotype in control injected larvae (Figure 53C).

Since the $m b p^{+}$ovoids were obvious at 8 hpi within the lysolecithin group, we decided to quantify the percentage of zebrafish presenting with myelin membrane changes within 
A

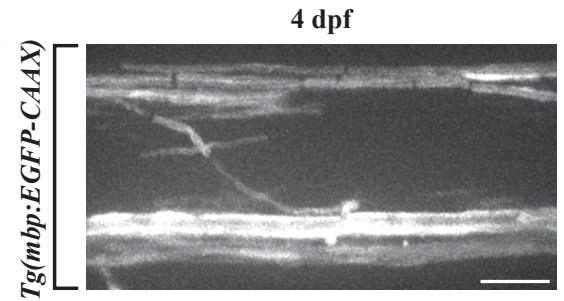

B

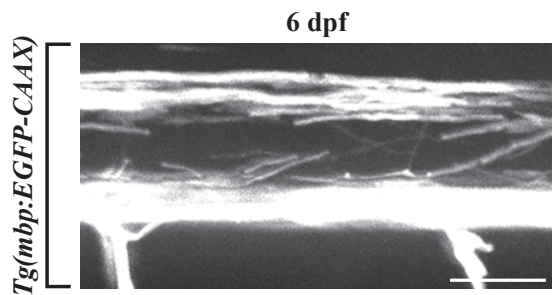

$\operatorname{Tg}(\operatorname{mbp}: E G F P-C A A X)$

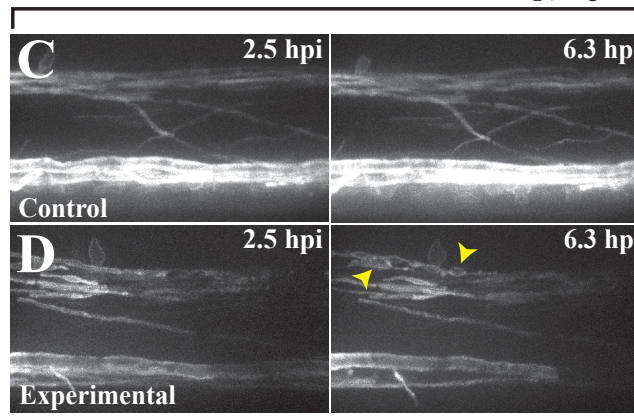

E

E Myelin Membrane Changes at 8 hpi

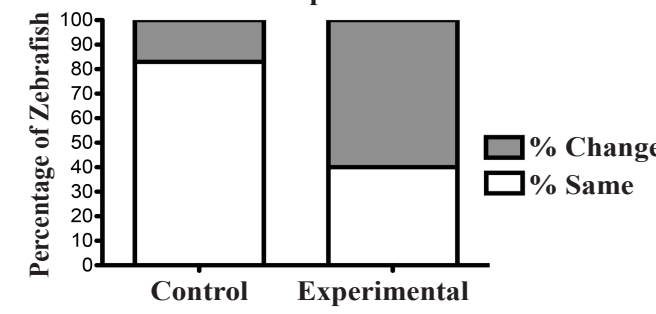

G

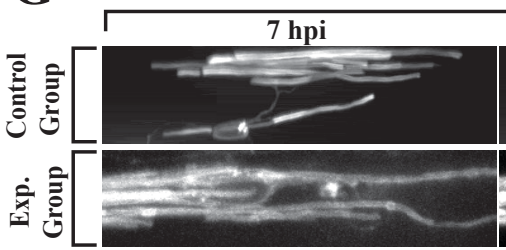

mbp:mCherry-CAAX

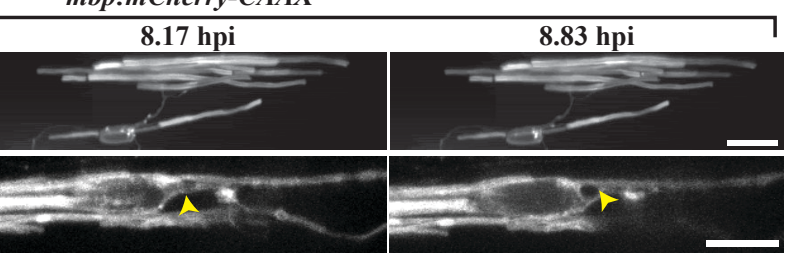

Figure 5-3. Myelin membrane changes within lysolecithin lesion. All images are lateral views of spinal cord with anterior to left and dorsal to top. (A \& B) Expression of $m b p: E G F P-C A A X$ appears sheath-like in the spinal cord of $4 \mathrm{dpf}(\mathrm{A})$ and $6 \mathrm{dpf}(\mathrm{B})$ zebrafish larvae. (C) Following injection of a control solution into the spinal cord of a $4 \mathrm{dpf} m b p: E G F P-C A A X$ larva, the $m b p^{+}$sheath structure remains unchanged. (D) Following injection of lysolecithin (experimental) into the spinal cord of a $4 \mathrm{dpf} m b p$ : EGFP-CAAX larva, the $m b p^{+}$sheath structures dynamically change to form ovoids (arrowheads). (E) Percentage of zebrafish with $m b p$ :EGFP-CAAX membrane changes at 8 hours post injection (hpi). A significantly greater percentage of zebrafish injected with lysolecithin (Experimental) had $\mathrm{mbp}^{+}$membrane changes as compared to zebrafish injected with control solution. (F) Average number of mbp:EGFP-CAAX ovoids observed 4 hpi -8 hpi demonstrates an increase in the average number of ovoids within the lysolecithin (Experimental) group. (G) F0 mbp:mCherry-CAAX zebrafish larvae injected with a control solution (Control, top) or lysolecithin (Exp. Group, bottom) at $6 \mathrm{dpf}$. Injection site was posterior to the mbp:mCherry-CAAX ${ }^{+}$oligodendrocyte such that the oligodendrocyte was anterior to the injection site but remained exposed to the solution. Following injection of a control solution (top), the oligodendrocyte remains relatively unchanged throughout the course of the time-lapse movie. In contrast, an ovoid-like structure is observed within the mbp:mCherry-CAAX ${ }^{+}$oligodendrocyte beginning around $8.17 \mathrm{hpi}$. Scale bars, $25 \mu \mathrm{m}$. 
the dorsal spinal cord at this time point, and observed a significant difference between control and experimental groups, with approximately $17 \%$ of control zebrafish with $\mathrm{GFP}^{+}$ myelin sheath membrane changes within the lesion area as compared to $60 \%$ of zebrafish injected with lysolecithin (Figure 5-3E) at 8 hpi. Additionally, we quantified the number of myelin ovoids within the control and experimental dispersal regions from 4 to 8 hpi, time-points where the $m b p^{+}$membrane is dynamically changing within the lysolecithininjected group. In these larvae, we observed an increase in the average number of $\mathrm{GFP}^{+}$ ovoids that formed (Figure 5-3F). Based on in vivo time-lapse imaging, the $m b p^{+}$ovoids in the lysolecithin group remained present throughout the duration of the 18.5 hour movie (data not shown).

Because $m b p: E G F P-C A A X$ is a stable transgenic line, it is difficult to visualize individual oligodendrocyte membrane changes within the dispersal region. Therefore, to investigate individual myelin sheath changes, we injected a plasmid for mbp:mCherry-CAAX (Mensch et al., 2015) into one-cell stage embryos to mosaically label $m b p^{+}$ oligodendrocytes in the spinal cord. At 6 dpf, we injected either a PBS control solution or lysolecithin posterior to an $m b p^{+}$oligodendrocyte, such that the oligodendrocyte would reside anterior to the injection site but would be exposed to the injected solutions (Figure 5-3G). Using in vivo imaging, we observed an obvious ovoid form at approximately 8.17 hpi within the lysolecithin group (Figure 5-3G, bottom), but this phenotype was not observed in the control injected larvae (Figure 5-3G, top). Taken together, our results demonstrate that lysolecithin induces oligodendrocyte membrane changes in the zebrafish spinal cord. 


\section{Axons are spared following lysolecithin injections}

In mammals, previous studies demonstrate that lysolecithin causes minimal damage to axons (Blakemore et al., 1977; Keough et al., 2015), which is a beneficial aspect of this model because demyelination is not occurring secondarily to axonal degeneration. To investigate if axons are spared in our focal demyelination model, we injected either a control solution or lysolecithin into the spinal cord of 4 dpf mbp:EGFPCAAX;cntn1b:mCherry (Almeida et al., 2011; Czopka et al., 2013) larvae to label myelin with membrane-tethered GFP and axons with cytosolic mCherry. Coupled with in vivo imaging, we observed that axons were spared within both the control (Figure 5-4A) and

the lysolecithin dispersal regions (Figure 5-4B) at 8 hpi, a time point in which we have observed active $\mathrm{mbp}^{+}$myelin changes via in vivo, time-lapse imaging (Figure 5-3D). From these data, we conclude that the effects observed on $m b p^{+}$myelin sheaths within the lysolecithin group described above are not secondary to axonal degeneration.

\section{Professional phagocytes are recruited to the injury site}

Professional phagocytes, including resident microglia of the CNS and infiltrating peripheral macrophages, respond to demyelination and clear debris in MS (Bø et al., 2012). However, controversy exists about the benefit of immune cells within MS lesions. For example, remyelination is impaired when macrophages are depleted early in the demyelination process (Kotter et al., 2001) and microscopy studies have identified macrophages within MS lesions (Bø et al., 2012), but how professional phagocytes maneuver throughout the CNS to clear myelin and axonal debris remains unknown. Additionally, macrophages and microglia have been reported to respond to $1 \%$ 


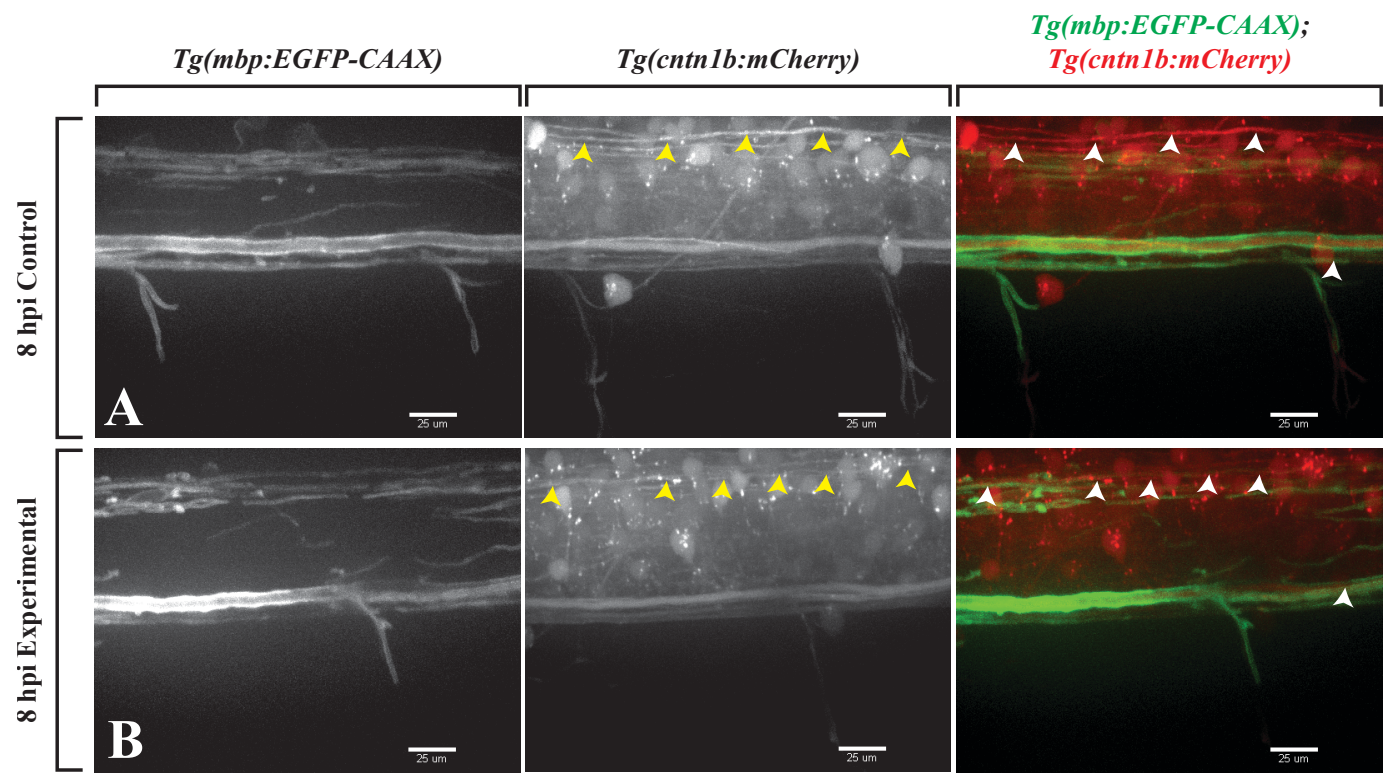

Figure 5-4. Axons are spared within the lysolecithin dispersal region. All images are lateral views of spinal cord with anterior to left and dorsal to top. Myelin membrane is labeled with the $m b p: E G F P-C A A X$ transgene and axons are labeled with the cntnlb:mCherry transgene. (A - B) $m$ Cherry $^{+}$axons (arrowhead) within the control (A) and within the lysolecithin (Experimental) (B) dispersal regions are spared at 8 hpi. Scale bars, $25 \mu \mathrm{m}$. 
lysolecithin injections in mammalian models and upon application to the adult zebrafish optic nerve (Kotter et al., 2001; Münzel et al., 2014). We next investigated if macrophages and microglia are recruited to the focal lesion in our demyelination model in zebrafish.

To investigate professional phagocyte recruitment in our model, we injected either a control solution or lysolecithin into the spinal cord of 4 and 6 dpf mbp:EGFPCAAX;mpeg1:mCherry (Almeida et al., 2011; Ellett et al., 2011) larvae, where myelinating oligodendrocytes are labeled with membrane GFP and macrophages are labeled with cytosolic mCherry. In both 4 and 6 dpf larvae, we observed macrophages move quickly throughout the CNS, maneuvering through $\mathrm{mbp}^{+}$myelin membrane layers. In an in vivo, time-lapse movie of a 4 dpf larva injected with lysolecithin, we observed maneuvering mCherry $^{+}$phagocytes appear to physically move the $m b p^{+}$membrane sheaths while traversing through the dispersal region within the spinal cord (Figure 5$5 \mathrm{~A})$. We next quantified the number of mpeg $^{+}$phagocytes recruited to the lesion area to determine if the lysolecithin group had a greater response by professional phagocytes. Following injection of a control solution or lysolecithin into the spinal cord of $4 \mathrm{dpf}$ mbp:EGFP-CAAX;mpegl:mCherry zebrafish larvae, we saw an increase in mpeg $^{+}$ phagocytes within the lysolecithin group ( $\mathrm{n}=6$ larvae) from 2 to 6 hpi as compared to control injections $(\mathrm{n}=6$ larvae) (Figure 5-5B). Taken together, the recruitment of professional phagocytes to the lesion area recapitulates the macrophage/microglia recruitment response that has been reported in mammalian lysolecithin models (Kotter et al., 2001; Münzel et al., 2014). 
A

Tg(mbp:EGFP-CAAX); Tg(mpeg1:mCherry)
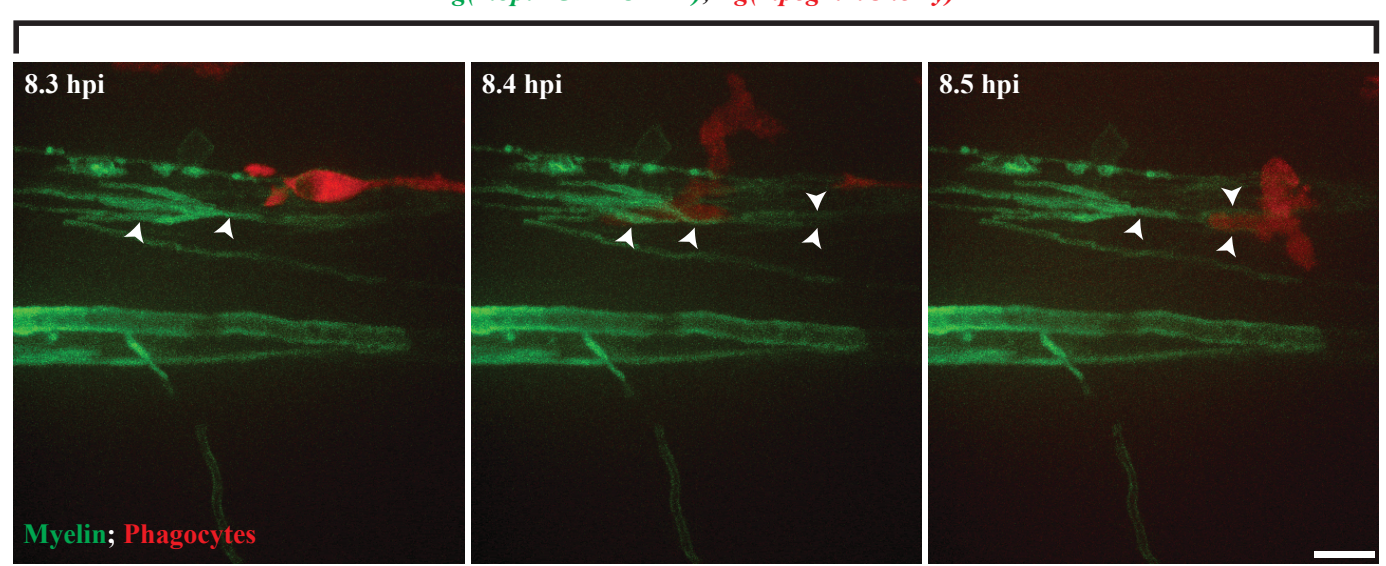

\section{B} Average \# of Professional Phagocytes within Dispersal Region

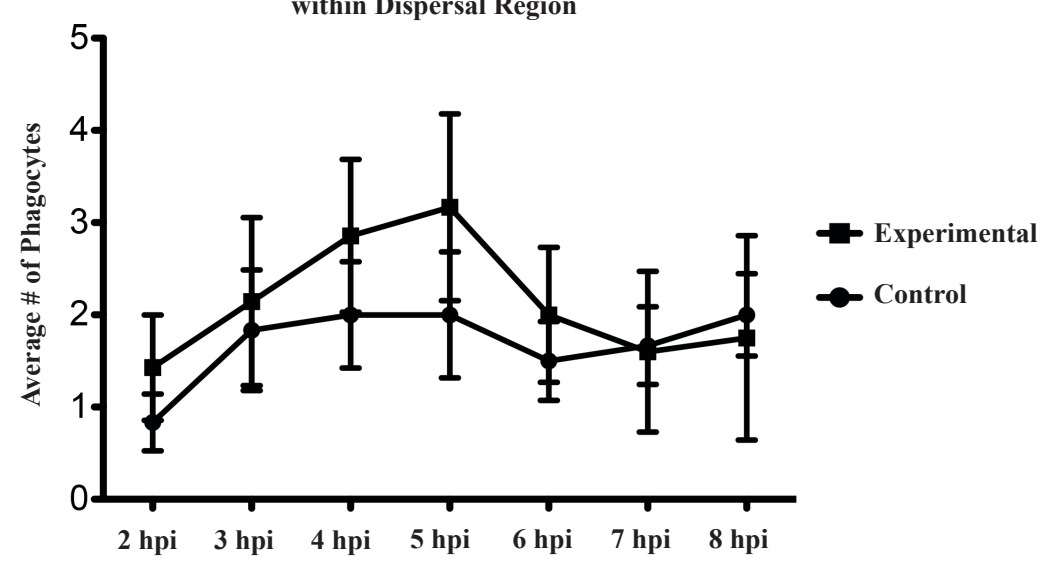

Figure 5-5. Professional phagocytes traverse through myelin layers. All images are lateral views of spinal cord with anterior to the left and dorsal to the top. Myelin membrane is labeled with $m b p: E G F P-C A A X$ transgene and professional phagocytes, i.e., microglia and macrophages, are labeled with mpeg1:mCherry transgene. (A) Following injection of lysolecithin, mCherry ${ }^{+}$ phagocyte is observed traversing through the mbp:EGFP-CAAX $X^{+}$myelin membrane layers within the lesion area, physically moving the layers (arrowheads). (B) Average number of professional phagocytes responding to the lesion following injection of a control (Control) or lysolecithin (Experimental) solution. Although there was a slight increase in the average number of $\mathrm{mpeg}^{+}$ phagocytes that responded to the lysolecithin, there were no significant differences between the two groups at all time points evaluated. Scale bars, $25 \mu \mathrm{m}$. 
In addition to phagocyte recruitment, macrophages and microglia proliferate during demyelination and inflammation (Chiang et al., 1996; Jenkins et al., 2011; Remington et al., 2007). To investigate if this occurs in our lysolecithin model, we injected lysolecithin into the spinal cord of 4 dpf mbp:EGFP-CAAX;mpeg1:mCherry zebrafish larvae and performed in vivo, time-lapse imaging. Intriguingly, we captured $\mathrm{mpeg}^{+}$cells proliferating near the lysolecithin dispersal region (Figure 5-6A), demonstrating professional phagocytes are both recruited to the lesion and proliferate in response to the demyelinating insult.

Because we observed myelin changes within the lysolecithin group, we next wanted to confirm that the professional phagocytes were responding to clear oligodendrocyte/myelin debris. To capture the clearance of oligodendrocyte debris in vivo, we injected lysolecithin into $6 \mathrm{dpf}$ sox10:mrfp;mpegl:gfp larvae and performed time-lapse imaging. As described above, mpeg $^{+}$cells were recruited to the lysolecithin dispersal region. By $3.67 \mathrm{hpi}$, we observed $\mathrm{mpeg}^{+}$cells with distinct cytoplasmic vacuoles surrounding $\operatorname{sox} 10^{+}$debris (Figure 5-6B). Taken together, our results demonstrate that our focal drug-induced demyelination model in zebrafish recapitulates the professional phagocyte response previously reported in lysolecithin injected mammalian models (Bø et al., 2012; Doring et al., 2015; Kotter et al., 2001; Kucharova et al., 2011; Rawji \& Yong, 2013) 
A

Tg(mbp:EGFP-CAAX); Tg(mpeg1:mCherry)

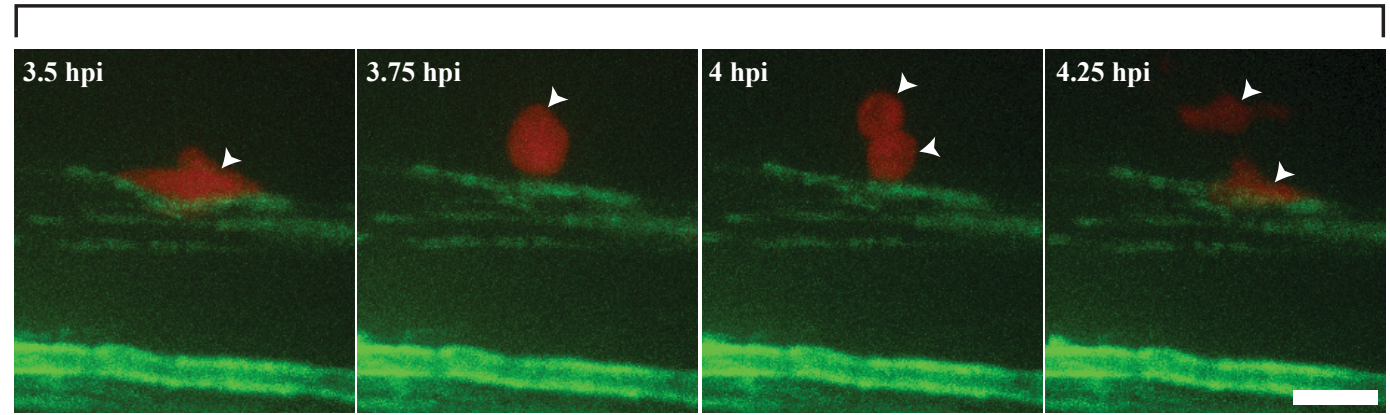

B

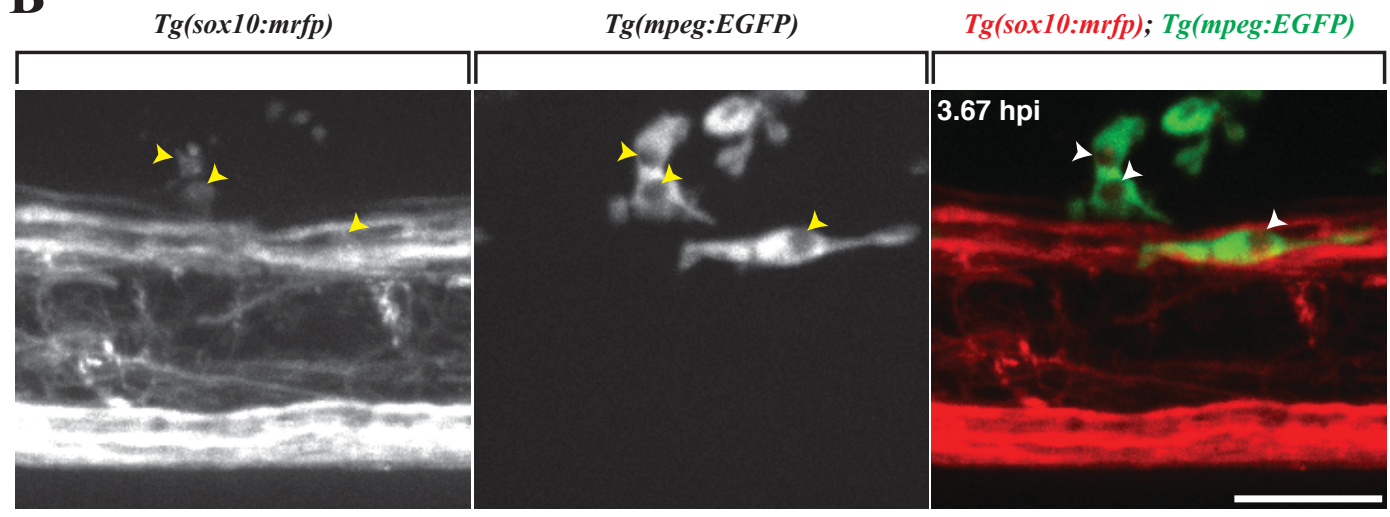

Figure 5-6. Professional phagocytes proliferate and clear Sox10 ${ }^{+}$debris. All images are lateral views of spinal cord with anterior to the left and dorsal to the top. (A) Myelin membrane is labeled with $m b p: E G F P-C A A X$ transgene and professional phagocytes, i.e., microglia and macrophages, are labeled with mpeg1:mCherry transgene. Following injection of lysolecithin into a $4 \mathrm{dpf}$ zebrafish spinal cord, a $m$ Cherry $^{+}$phagocyte recruited to the lysolecithin lesion proceeds to proliferate near the dispersal region. Following proliferation, one of the cells returns to the mbp:EGFP-CAAX ${ }^{+}$membrane layers. (B) Oligodendrocyte membrane is labeled with sox 10:mrfp transgene and professional phagocytes, i.e., microglia and macrophages, are labeled with mpeg1:EGFP transgene. Following injection of lysolecithin into a $6 \mathrm{dpf}$ zebrafish spinal cord, sox $10^{+}$debris is observed within mpeg $^{+}$cell vacuoles (arrowheads). Scale bars, $25 \mu \mathrm{m}$. 


\section{Oligodendrocyte cytoskeleton dynamics following exposure to a demyelinating insult}

Actin dynamics have recently been identified as essential for myelin sheath formation (Nawaz et al., 2015; Samanta \& Salzer, 2015; Zuchero et al., 2015). Specifically, filamentous actin (F-actin) is integral for initiating myelin wrapping and, upon completion of myelination, F-actin is disassembled from the inner tongue/leading edge and becomes localized to the lateral edges of the myelin sheath (Nawaz et al., 2015; Samanta \& Salzer, 2015; Zuchero et al., 2015). Interestingly, actin polymerization is involved in peripheral myelin fragmentation and ovoid / onion bulb formation following injury in vitro, and inhibiting actin polymerization prevents myelin ovoid formation (Jung et al., 2011). Furthermore, cytoskeletal plasticity was recently identified as an important component of demyelination, suggesting that mechanisms within the oligodendrocyte cytoskeleton may be actively involved in reacting to demyelinating insults (Locatelli et al., 2015). Specifically, Locatelli et al. (2015) observed an increase in $\beta$-actin and $\beta$ tubulin gene transcription in response to diphtheria-toxin mediated oligodendrocyte death and in response to autoimmune inflammation via EAE (Locatelli et al., 2015).

To investigate normal actin dynamics within $\operatorname{sox} 10^{+}$oligodendrocytes in vivo, we imaged non-injected 4, 5 and 6 dpf sox10:gal4;UAS:Lifeact-GFP larvae (Helker et al., 2013) (Figure 5-7A). At all stages evaluated, we observed $\mathrm{GFP}^{+}$F-actin arranged as sheath-like structures, reminiscent of the appearance of $m b p^{+}$myelin membrane sheaths. However, in vivo, time-lapse imaging of $4 \mathrm{dpf}$ larvae revealed that the $\mathrm{GFP}^{+}$actin in the oligodendrocyte cytoskeleton was much more dynamic at 4 dpf when compared to $6 \mathrm{dpf}$. At $6 \mathrm{dpf}$, the $\mathrm{GFP}^{+}$F-actin was significantly less dynamic and stable (Figure 5-7A). 
Because of these observations, we decided to inject lysolecithin into $6 \mathrm{dpf}$ sox 10:Gal4;UAS:Lifeact-GFP larvae, a time-point where the $\mathrm{GFP}^{+}$sheath-like structures were not as dynamic. At 2 hpi, $\mathrm{GFP}^{+}$F-actin within $\operatorname{sox} 10^{+}$cells appeared sheath-like. However, by 4 hpi we began observing dynamic changes (Figure 5-7B). The $\mathrm{GFP}^{+} \mathrm{F}-$ actin rearranged from a sheath-like structure to form obvious ovoid-like structures by 8.33 hpi (Figure 5-7B). The arrangement of the F-actin into ovoid-like structures is reminiscent of the $m b p^{+}$membrane changes described above, and occurs at the same time points post-injection. These preliminary results demonstrate that the dynamic responses from components of the oligodendrocyte cytoskeleton appear to be involved in demyelination.

\section{Clemastine fumarate minimizes the effects of myelin membrane changes following exposure to lysolecithin}

In a high-throughput micropillar array screen, clemastine fumarate was identified as a compound that significantly promoted oligodendrocyte differentiation (Mei et al., 2014), and further investigations confirmed that clemastine is a potential candidate for promoting remyelination in mammalian systems (Mei et al., 2014). Clemastine is an antimuscarinic compound that received FDA approval as a first-generation antihistamine. Its in vivo remyelination capabilities were investigated by injecting lysolecithin into the spinal cord of adult mice that had been treated with clemastine via oral gavage before lysolecithin exposure (Mei et al., 2014). Mice receiving clemastine had accelerated remyelination, as evidenced by an increase in oligodendrocyte differentiation and 
A

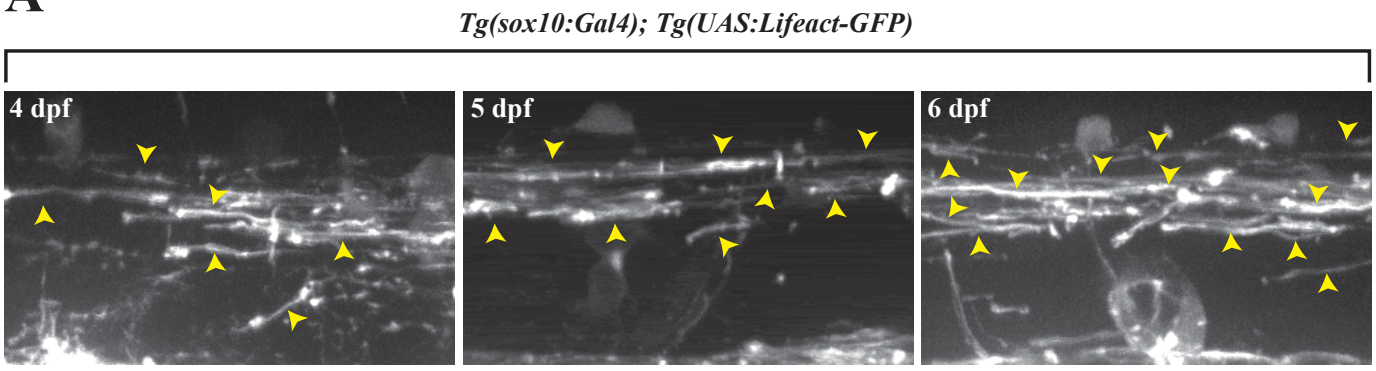

B

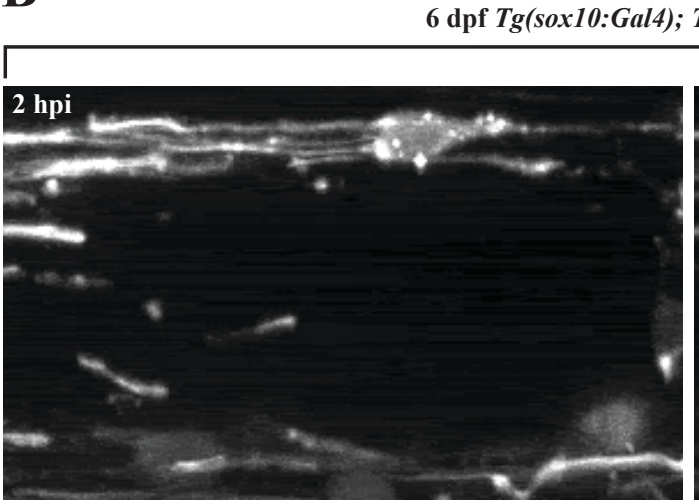

$6 \mathrm{dpf} \operatorname{Tg}($ sox 10:Gal4); $\operatorname{Tg}($ UAS:Lifeact-GFP)
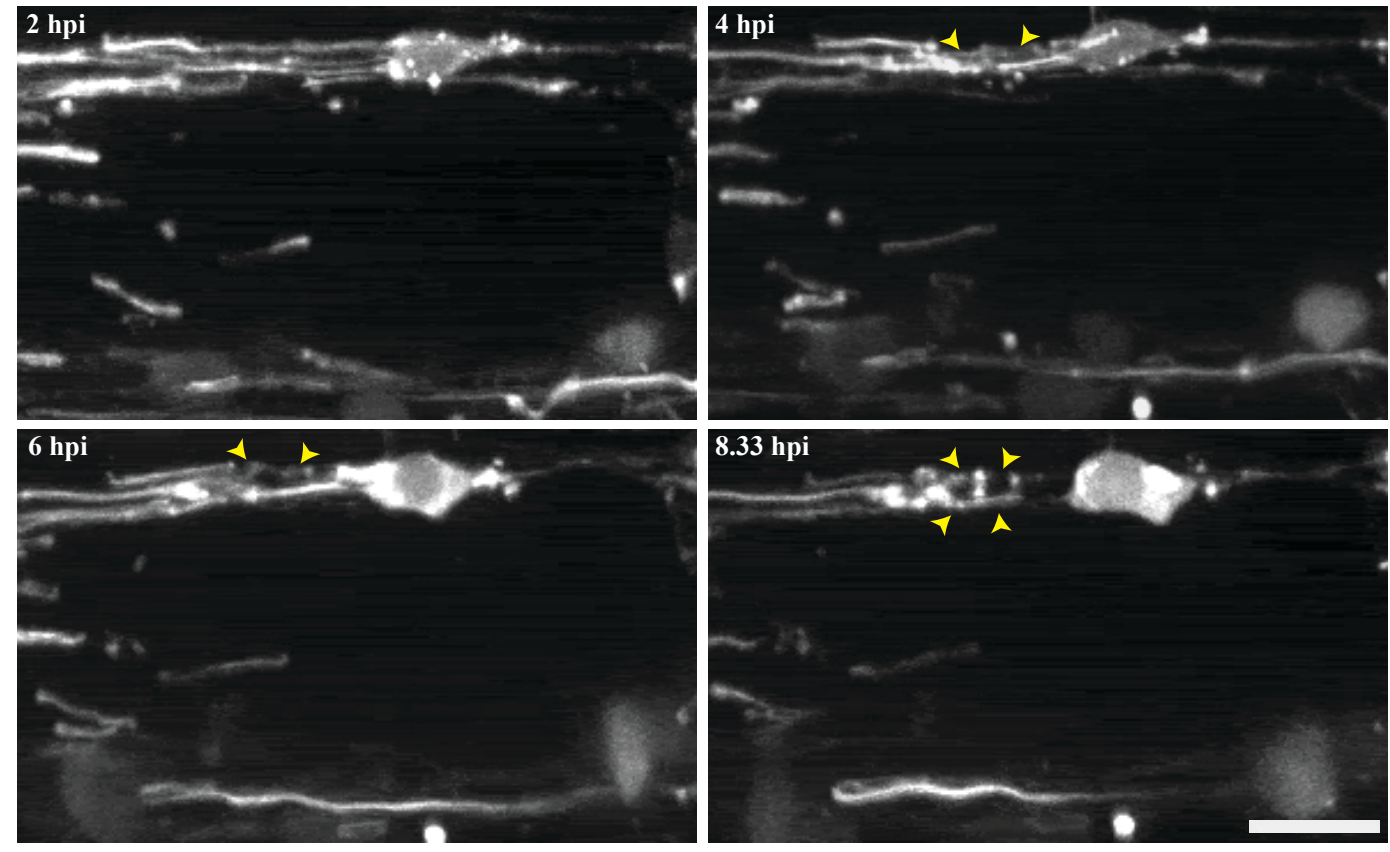

Figure 5-7. Oligodendrocyte cytoskeletal components dynamically change following exposure to lysolecithin. All images are lateral views of spinal cord with anterior to the left and dorsal to the top. (A) In vivo imaging of sox10:Gal4;UAS:Lifeact-GFP larvae at $4 \mathrm{dpf}, 5 \mathrm{dpf}$ and $6 \mathrm{dpf}$ demonstrate $\mathrm{GFP}^{+} \mathrm{F}$-actin is arranged in sheath-like structures (arrowheads). At 4 dpf, the $G F P^{+}$ F-actin is much more dynamic than at $6 \mathrm{dpf}$. (B) Following injection of lysolecithin into a $6 \mathrm{dpf}$ sox 10:Gal4;UAS-Lifeact-GFP zebrafish spinal cord, in vivo time-lapse imaging reveals that $\mathrm{GFP}^{+}$ F-actin is observed to dynamically change (arrowheads) from a sheath-like arrangement (2 hpi) to forming ovoid-like structures (8.33 hpi). Scale bars, $25 \mu \mathrm{m}$. 
enhanced myelin thickness as compared to lysolecithin only injected controls (Mei et al., 2014).

Because clemastine has previously been used as an agent for promoting remyelination in a lysolecithin focal demyelination mouse model, we decided to investigate the effects of clemastine in our zebrafish model. We injected a lysolecithin cocktail including either $500 \mathrm{nM}$ or $1 \mathrm{uM}$ of clemastine fumarate into the spinal cord of 4 dpf mbp:EGFPCAAX;mpeg1:mCherry zebrafish (Figure 5-8). Excitingly, we observed a decrease in the percentage of zebrafish experiencing myelin membrane changes within the lysolecithin + $500 \mathrm{nM}$ clemastine cocktail dispersal region (Figure 5-8C) and lysolecithin $+1 \mathrm{uM}$ clemastine cocktail dispersal region (Figure 5-8B) as compared to injections with only lysolecithin (Figure 5-8A) at 8 hpi (Figure 5-8D). The same trend was observed when we quantified the number of ovoids at 4, 6 and 8 hpi. We observed a decrease in the average number of ovoids in $4 \mathrm{dpf}$ larvae injected with lysolecithin $+500 \mathrm{nM}$ Clemastine $(\mathrm{n}=3$ zebrafish) and lysolecithin $+1 \mathrm{uM}$ Clemastine $(\mathrm{n}=2$ zebrafish) as compared to lysolecithin injections alone ( $\mathrm{n}=6$ zebrafish) (Figure 5-8E) at all time points evaluated. Thus, treating with clemastine has an effect on either preventing the demyelinating membrane changes or by increasing the remyelination kinetics following a demyelinating insult in zebrafish.

Taken together, these results validate our focal drug induced demyelination model as a viable assay for visualizing mechanics underlying myelin breakdown in vivo. 


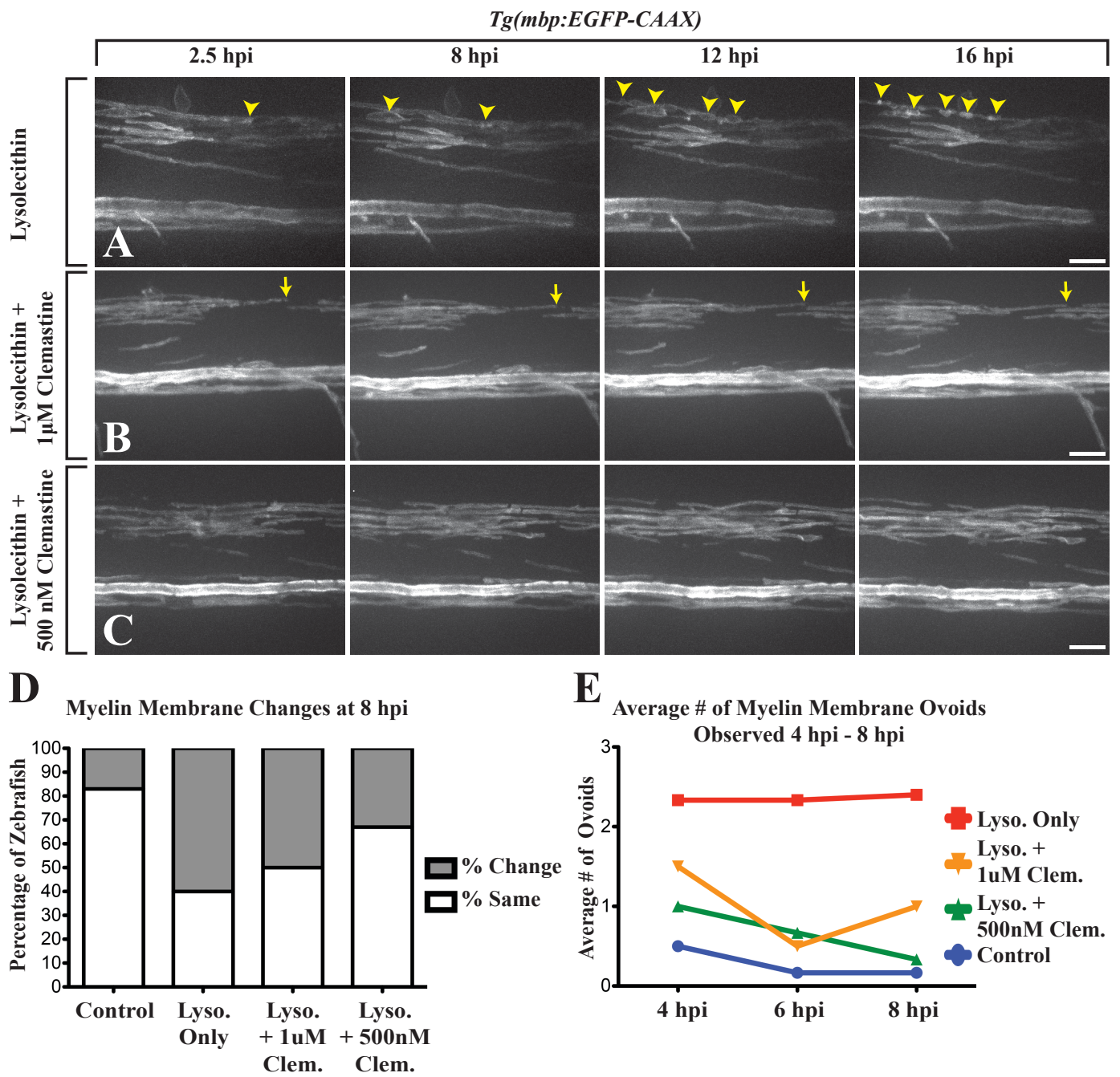

Figure 5-8. Clemastine reduces myelin membrane changes following a demyelinating insult. All images are lateral views of spinal cord with anterior to the left and dorsal to the top. (A) Injecting lysolecithin into $4 \mathrm{dpf} m b p$ :EGFP-CAAX zebrafish spinal cords results in $m b p^{+}$ membrane changing from sheath-like to forming ovoids (arrowheads). (B) Injecting lysolecithin with $1 \mu \mathrm{M}$ Clemastine into $4 \mathrm{dpf} m b p: E G F P-C A A X$ zebrafish spinal cords results in $m b p^{+}$ membrane remaining as sheath-like. A $m b p^{+}$sheath is observed extending anterior to posterior (arrow). (C) Injecting lysolecithin with $500 \mathrm{nM}$ Clemastine into $4 \mathrm{dpf} m b p: E G F P-C A A X$ zebrafish spinal cords results in $m b p^{+}$membrane remaining as sheath-like. (D) A significantly greater percentage of $m b p: E G F P-C A A X$ zebrafish injected with lysolecithin (Experimental) had $m b p^{+}$ membrane changes at 8 hpi as compared to zebrafish injected with control. There is an increase in the percentage of zebrafish with unchanged $\mathrm{mbp}^{+}$membranes that remained sheath-like at $8 \mathrm{hpi}$ when $1 \mu \mathrm{M}$ or $500 \mathrm{nM}$ Clemastine was included in the injection cocktail. (F) Average number of mbp:EGFP-CAAX ovoids observed $4 \mathrm{hpi}-8 \mathrm{hpi}$. Injections including $1 \mu \mathrm{M}$ or $500 \mathrm{nM}$ Clemastine within lysolecithin cocktail decreased the average number of ovoids observed at all time points evaluated as compared to injections with lysolecithin alone. Scale bars, $25 \mu \mathrm{m}$. 


\section{Discussion}

\section{Animal models for recapitulating the heterogeneity of MS}

MS presents with remarkable heterogeneity, ranging from clinical symptoms, radiological appearances, histopathological lesion patterns, response to therapy and potentially a multi-factorial etiology (Lassmann et al., 2001; Lucchinetti et al., 2000; Stys et al., 2012). MS has traditionally been classified as an autoimmune-mediated neuroinflammatory disease in which the immune system becomes primed and T-cells infiltrate into the CNS to attack myelin. Pathological samples from patients demonstrate certain lesion patterns with demyelination and inflammatory infiltration by $\mathrm{T}$ cells and macrophages (Frohman et al., 2006; Lucchinetti et al., 2000; Noseworthy et al., 2000), supporting a neuroinflammatory etiology or pathophysiology classified under the “outside-in" model of MS etiology (Stys et al., 2012). However, because of the vast heterogeneity, certain histopathological samples also support that the initial trigger or early lesion may be caused by oligodendrogliopathy or myelin defects (Barnett \& Prineas, 2004; Lucchinetti et al., 2000; Stys et al., 2012), supporting an "inside-out" model of MS etiology (Stys et al., 2012). Recently, an oligodendrocyte ablation mouse model (Traka et al., 2010) demonstrated that an initial demyelination event, including oligodendrocyte death, is sufficient for triggering a later-onset T-cell mediated demyelinating disease (Traka et al., 2015), further suggesting that a multi-factorial etiology for MS may exist (Stys et al., 2012). Because of this, it is imperative that various animal models are used to recapitulate the heterogeneous aspects of MS and its symptoms. 
One animal model that has been instrumental in developing a better understanding about the autoimmune neuroinflammatory aspects associated with the disease and for investigating "outside-in" hypotheses for MS etiology is the experimental autoimmune encephalomyelitis (EAE) model. EAE lesions resemble MS pathology (Ransohoff, 2012; Waksman \& Adams, 1962) and, if the SJL/J mouse strain is used, relapses can be produced (Dal Canto et al., 1995). However, EAE is commonly induced in C57Bl/6 mice because of the plethora of genetic strains and resources available, but the disease course in this strain is monophasic, lacking relapses or remyelination (Ransohoff, 2012). Additionally, the EAE model has poorly predicted the therapeutic success of potential treatment strategies for MS (Ransohoff, 2012).

The limitations of the EAE model, in combination with the diverse histopathology of MS lesions, including patterns or subtypes in which oligodendrocyte or myelin sheath cytodegeneration appears to be the initial trigger of the disease (Lucchinetti et al., 2000; Stys et al., 2012), supports the need for non-inflammatory demyelination models. The use of toxin-induced demyelination models have been imperative for investigating mechanics underlying oligodendrogliopathies, or "inside-out" etiologies, as well as for processes involved in remyelination (Blakemore et al., 1977; Blakemore \& Franklin, 2008; Foote \& Blakemore, 2005; Ransohoff, 2012). Although toxin-induced demyelination models lack an ongoing immune response, there is a unique advantage of being able to separate demyelination events from processes underlying remyelination (Miller \& Fyffe-Maricich, 2010; Ransohoff, 2012). Because of this spatiotemporal predictableness, toxin induced 
demyelination models can also be useful for screening therapeutic strategies that promote remyelination (Ransohoff, 2012).

Mammalian models commonly used to study MS, including EAE and toxin-induced demyelination models, as well as clinical samples are not amenable for investigating mechanics and processes underlying myelin destruction and subsequent repair in vivo. To overcome this limitation, we created our focal drug-induced demyelination model in zebrafish that allows us to visualize myelin destruction in a live, intact vertebrate model system, and investigate mechanics and cellular interactions involved in myelin breakdown and repair. Future studies using our focal drug induced demyelination model as a first-pass drug screening tool are needed for investigating the safety and efficacy of novel compounds that either inhibit myelin breakdown, or promote its repair, to potentially reveal therapeutic strategies for treating MS.

\section{Observing myelin membrane changes in vivo}

Myelin membrane changes, such as the formation of myelin ovoid-like or onion bulb structures, are characteristic of myelin degradation (Acar et al., 2004; Prineas \& Connell, 1979). These ovoid-like structures have also been described as intramyelinic edema (Kaufmann et al., 2012; McMartin et al., 1997) or vacuoles (Triarhou \& Herndon, 1985) and contain edema fluid. This fluid accumulation causes the myelin to separate along the intraperiod lines (Hirano \& Llena, 2006; Hirano et al., 1965; Kaufmann et al., 2012; McMartin et al., 1997), which is a structure comprising of opposing external layers of myelin membrane that contact one another (Peters, 1960; Siegel et al., 1999). These 
vacuoles can be identified in mammals using the following techniques: light microscopy shows separation of the myelin lamellae, ultrastructural studies demonstrate myelin lamellae splitting along the intraperiod line and there is less Hematoxylin and Eosin (H\&E) staining along regions of the myelin sheath containing intramyelinic vacuoles (Hirano \& Llena, 2006; Kaufmann et al., 2012; McMartin et al., 1997).

Intramyelinic vacuoles and fluid accumulation within the myelin sheath have been reported following lysolecithin-induced demyelination (Triarhou \& Herndon, 1985) and also in other toxin induced demyelination models (Blakemore, 1973; 1974). In our focal demyelination model, we are able to visualize the formation of these vacuoles in vivo as they form in real time. However, future studies are needed to confirm that the ovoids we see in our in vivo imaging assays are from intramyelinic edema, i.e., ultrastructural data demonstrating separation of myelin lamellae along the intraperiod line (Hirano et al., 1965; Hirano \& Llena, 2006; Kaufmann et al., 2012) and H\&E staining to confirm fluid accumulation within the vacuoles (McMartin et al., 1997).

Alternatively, myelin ovoids have been described as digestion chambers for degenerating axons (Griffin et al., 1990; McMartin et al., 1997). However, these ovoids may also form as a result of the myelin sheath breaking down (Kaufmann et al., 2012; McMartin et al., 1997). An advantage of the lysolecithin drug-induced demyelination model is that axons are generally spared (Arnett et al., 2004; Blakemore \& Franklin, 2008; McKay et al., 1998). The myelin ovoids we observe following a lysolecithin insult in our focal demyelination model are presumably due to myelin sheath breakdown and not secondary 
to axonal degeneration. However, future studies evaluating the ultrastructure of the drug dispersal region are needed to confirm the ovoids are resulting from myelin sheath breakdown due to fluid accumulation separating the myelin lamellae.

Additionally, the dynamic rearrangement of filamentous actin within the oligodendrocyte cytoskeleton suggests actin polymerization may be involved with the formation of the ovoids. Actin dynamics are essential for myelin sheath formation and it is reasonable to assume it has an integral role in kinetics underlying de- and re-myelination. Following in vitro peripheral nerve injuries, actin polymerization is involved in the formation of onion bulbs as inhibiting actin polymerization prevents the ovoid or onion bulb from forming (Jung et al., 2011), demonstrating that actin polymerization is actively involved in myelin membrane sheath changes. Furthermore, mechanisms within the oligodendrocyte cytoskeleton may be actively involved in reacting to demyelinating insults as $\beta$-actin and $\beta$-tubulin gene transcription are upregulated in response to diphtheria-toxin oligodendrocyte death and EAE autoimmune inflammation (Locatelli et al., 2015). To our knowledge, there have been no reports on actin dynamics following lysolecithininduced demyelination. Thus, future studies are needed in our model of demyelination to determine if actin dynamics are actively rearranging the myelin sheath to form these ovoids, or are simply a passive bystander that dynamically rearranges as a result of the myelin sheath changes. 


\section{Professional phagocyte response following the demyelinating insult}

Professional phagocytes, including resident microglia of the CNS and infiltrating peripheral macrophages, are thought to mediate demyelination and clear debris in MS (Bø et al., 2012). The removal of myelin debris has been identified as a key step in promoting myelin repair (Kotter et al., 2006; Neumann et al., 2008), and remyelination is significantly impaired when macrophages are depleted early in the repair process (Kotter et al., 2001; Kotter et al., 2005) or when there is inefficient myelin debris clearance by microglia (Lampron et al., 2015). Although a recent paper demonstrated that infiltrating monocyte-derived macrophages were responsible for initiating demyelination and resident microglia were responsible for clearing myelin debris (Yamasaki et al., 2014), the primary functions of macrophages and microglia in de- and re-myelination are not well understood.

Previously, it has been reported that there is rapid recruitment of macrophages and microglial cells following injection of lysolecithin in to the mouse spinal cord (Ousman \& David, 2000). Phagocytes are observed within the injection region by 6 hours post injection and ultrastructural analysis at 4 dpi reveals macrophages containing myelin debris (Ousman \& David, 2000). Microglia/macrophages have also been shown to proliferate extensively in early demyelination (Matsumoto et al., 1992; Raivich \& Banati, 2004; Schönrock et al., 1998) as well as in the EAE model (Rinner et al., 1995) and lysolecithin-induced demyelination in primates (Dousset et al., 1995). In the adult zebrafish optic nerve exposed to a $1 \%$ lysolecithin gel foam, there is an increase in the number of professional phagocytes at both 4 and 8 days post lesion (Münzel et al., 2014). 
In our model of demyelination, we also observe $m p e g 1^{+}$professional phagocytes respond to the injection region quite rapidly. The $m p e g 1^{+}$professional phagocytes are recruited to the lesion and have the capability to proliferate, as previously reported in a primate model of lysolecithin-induced demyelination (Dousset et al., 1995). Since lysolecithin acts as a chemotactic factor for peripheral macrophages (Quinn et al., 1988), we expected to see an increase in the number of phagocytes within the lysolecithin lesion. Although there was an increase in the average number of professional phagocytes we observed from $2 \mathrm{hpi}-6$ hpi, the results were not statistically significant as we observed great variability both within and between groups in regards to the number of $m p e g l^{+}$professional phagocytes that were recruited. Future studies are needed to increase the sample size in both the experimental and control groups to provide an accurate assessment of the number of phagocytes responding to the injection site alone or to the lysolecithin induced demyelination, and also to provide a better understanding about the temporal dynamics and response behaviors by professional phagocytes.

Phagocytes have been described as having both beneficial and detrimental roles in MS (Arnett et al., 2001; Bitsch et al., 2000; Copelman et al., 2001; Domingues et al., 2016; Hill et al., 2004; Hinks \& Franklin, 2000; Huitinga et al., 1990; Kotter et al., 2001; 2005; Lampron et al., 2015; Lassmann et al., 2001; Mason et al., 2001; Nathan, 1987; Ousman \& David, 2000; Rose et al., 2004). Zebrafish offer a unique advantage for investigating the transition from beneficial phagocytic responses to detrimental behaviors in vivo as macrophages can be depleted using a nitroreductase genetic model (Curado et al., 2008; Pisharath \& Parsons, 2009; Pisharath et al., 2007), allowing for inducible and reversible 
depletion of mpegl $1^{+}$cells. E. coli nitroreductase can be expressed under the control of the mpeg1 promoter (Palha et al., 2013; Travnickova et al., 2015) by crossing the following zebrafish lines to generate embryos positive for both transgenes: $T g(m p e g 1: G a l 4 F F)^{g l 25}$ (Ellett et al., 2011) and $\operatorname{Tg}\left(U A S: n f_{S} B-m C h e r r y\right)^{c 26}$ (Davison et al., 2007). To initiate cell depletion, larvae are immersed in the prodrug metronidazole and nitroreductase converts the metronidazole into a cytotoxin, resulting in cell death. The effects are reversible upon removing the zebrafish from the prodrug solution. This genetic ablation model allows for spatial and temporal control over cell ablation and can be used in our focal demyelination model to investigate the impact that the absence of mpegl $l^{+}$cells has on de- and remyelination events in real-time, in a living vertebrate organism, a feat mammalian systems currently cannot offer. These types of experiments will provide us with a better understanding about the roles and temporal dynamics of professional phagocytes in myelin breakdown and repair.

\section{Clemastine fumarate promotes myelin repair}

A high-throughput screen identified FDA-approved clemastine fumarate as an effective drug for accelerating remyelination following a demyelinating insult (Mei et al., 2014). Clemastine received FDA approval as an antihistamine as it blocks the histamine H1 receptor. However, its success for accelerating remyelination following a demyelinating insult in mice (Mei et al., 2014) is likely due to its anticholinergic effects of antagonizing M1/M3 muscarinic acetylcholine receptors (Harlow et al., 2015). Oligodendrocytes have been shown to express M1 - M4 muscarinic receptor subtypes; however, OPCs demonstrate greater expression of M3, M1 and M4 subtypes whereas in mature 
oligodendrocytes, the expression of M1, M3 and M4 subtypes is down-regulated (De Angelis et al., 2012). Thus, oligodendrocyte differentiation is inhibited when muscarinic receptors are activated (De Angelis et al., 2012; Harlow et al., 2015). Because of this, enhanced remyelination by antimuscarinic drugs like clemastine is proposed to occur by impacting oligodendrocyte differentiation directly (Kremer et al., 2015).

To further validate that our focal demyelination model recapitulates what has been described in mammalian systems, we included $500 \mathrm{nM}$ or $1 \mu \mathrm{M}$ of Clemastine as part of the lysolecithin injection cocktail. Excitingly, we observed a reduction in the percentage of zebrafish experiencing $m b p^{+}$membrane changes as well as the average number of $m b p^{+}$ovoids as compared to lysolecithin only injected fish. These results also demonstrate the capability of our focal demyelination model to be used as a drugscreening assay for therapeutics that potentially inhibit myelin breakdown or promote its repair.

In conclusion, the lysolecithin induced demyelination model we developed using zebrafish larvae recapitulates phenotypes that have been described in mammalian systems following injection of lysolecithin, with a major advantage of being able to visualize mechanics underlying de- and re-myelination in vivo, a feat that is currently not possible in mammalian models. This focal demyelination model may become a useful drugscreening assay for revealing therapeutic strategies that prevent myelin breakdown or accelerate and enhance remyelination kinetics. 
Acknowledgements: We would like to thank members of the Kucenas laboratory for valuable discussions and Lori Tocke for zebrafish care. We would also like to thank Dr. Alban Gaultier and Anthony Fernandez-Castaneda from UVA and Drs. David Lyons, Hae-Chul Park and Erik Herzog for sharing expertise, zebrafish lines, constructs and reagents. This work was supported by the National Institutes of Health (NIH): NS072212 (SK). 


\section{Chapter 6}

\section{Discussion and future directions}

\section{Summary}

In this dissertation, I present findings regarding glial cell plasticity in development and in response to myelinopathies by focusing on glial-glial interactions during PNS spinal motor nerve assembly, response to injury and nerve maintenance as well as myelinating glial cell responses following a demyelinating insult in the CNS. By using zebrafish, I was able to visualize glial-glial interactions in the PNS and glial cell responses in the CNS. However, in vivo imaging of transgenic zebrafish does not provide the necessary resolution to view ultrastructural morphologies. Because of this, I modified a transmission electron microscopy (TEM) protocol, described in Chapter 3, and was able to efficiently identify nervous system structures to investigate glial cell ultrastructure. Although this TEM protocol focused on spinal motor nerves, it can be adapted to locate other discrete, repeated nervous system structures, including within the CNS, as well as during different time points and zebrafish developmental stages.

To better characterize peripheral glial-glial interactions in motor nerve assembly, maintenance and injury responses, in Chapter 4, we combined in vivo imaging, a laser axonal transection assay in collaboration with Dr. Wendy Lewis, TEM and immunohistochemistry techniques to investigate perineurial glial plasticity in response to a peripheral myelinopathy in zebrafish. The $e r b b 3 b^{\text {st48-/- }}$ zebrafish were used as a model because the peripheral myelinating glial cells, including MEP glia and Schwann cells, fail 
to associate with motor axons and OPCs exit the spinal cord, associate with and appear to ensheath peripheral axons. Interestingly, early perineurial glial developmental behaviors are unaltered, i.e., perineurial glia exit the spinal cord, proliferate and extend and retract in manners comparable to wildtype controls. However, perineurial morphology is altered along certain nerves, and an altered morphology phenotype persists in adult zebrafish, as investigated using TEM. Furthermore, perineurial glial injury responses are compromised after injuring an axonal tract that contains ectopic OPCs. Perineurial differentiation is also altered, as evidenced by the unusual distribution of increased ZO-1 labeling and tight junctional pockets in $e r b b 3 b^{-/}$, resulting in a compromised and leaky barrier. Although perineurial glial cells demonstrate plasticity for certain developmental and maturation behaviors, results from our study further support the importance of peripheral glial-glial interactions in forming and maintaining a functional nerve.

In addition to peripheral glial-glial interactions, Chapter 5 presents findings using a novel focal demyelination model in zebrafish that allows us to visualize oligodendrocyte response following a demyelinating insult. By injecting lysolecithin, a membrane solubilizing agent that has been injected into the nervous system of mammalian models to induce demyelination, we observe processes associated with myelin damage, including a reduction in Sox $10^{+}$oligodendrocytes, recruitment of macrophages / microglia and the formation of ovoids or vacuole structures within the myelin membrane. This model may be useful for screening potential therapeutic compounds for treating demyelinating disorders like MS, and therefore, has implications beyond visualizing myelin breakdown and repair in vivo. 
Taken together, this dissertation has enhanced our understanding about the plasticity and responses of glial cells, and further supports the need for investigating the role of glial interactions and behaviors during nervous system development, maintenance and in response to damage.

\section{Heterogeneity of glial subtypes}

The nervous system contains several types of glial cells. Peripheral myelinating glia, including Schwann cells and MEP glia, as well as perineurial glia represent the glial subtypes of the PNS. Within the mammalian CNS, oligodendrocytes, astrocytes and microglia comprise the main glial cell populations. An additional complexity to these various glial cell populations is that there is heterogeneity within the subtypes, as described below.

In the zebrafish PNS, peripheral myelinating glia include Schwann cells (Jessen \& Mirsky, 1991; 1997; 1998; 1999; Mirsky et al., 2002) and MEP glia (Smith et al., 2014). Schwann cells consist of both myelinating and non-myelinating subtypes that are derived from the neural crest (Jessen \& Mirsky, 1991; 1997; Le Douarin et al., 1991; Raible et al., 1992), whereas MEP glia are derived from the pMN domain of the spinal cord and migrate into the periphery via MEP transition zones to associate with and myelinate ventral motor roots (Smith et al., 2014). Since MEP glial cells were a relatively recent discovery (Smith et al., 2014), there is much to be learned about this cell population and future studies should be conducted identifying markers that are specific to this cell population. Myelinating Schwann cells have been shown to demonstrate remarkable 
heterogeneity and secrete growth factors with differential expression patterns depending on the type of axons, i.e. motor versus sensory (Höke et al., 2006; Jesuraj et al., 2011), and the location along the axon, i.e., proximal versus distal (Brushart et al., 2013). In addition to peripheral myelinating glial heterogeneity, recent evidence in mouse suggests there is heterogeneity amongst perineurial glial cells, as only a subpopulation of perineurial cells are $N k x 2.2 a^{+}$and derived from the CNS (Clark et al., 2014). Thus, subpopulations of perineurial cells may play different or distinct roles in motor nerve development, injury responses and compensating for myelinopathies. To develop a better understanding about the heterogeneity of perineurial cells, Next Generation Sequencing, including quantitative single-cell RNA-seq, is needed to reveal new markers. Identifying new markers is imperative for investigating the location of, function and the diverse roles these different subpopulations may be involved in during nervous system development and maintenance.

The CNS also consists of several glial cell populations. Macroglia cells, including astrocytes and oligodendrocytes, are derived from the CNS neuroepithelium whereas microglial cells are haematopoietic (Rowitch \& Kriegstein, 2010). Astrocytes play important roles both during development and in disease states and regulate ion distribution, water balance, synapse formation, synapse function, synapse plasticity and are involved in maintaining the integrity of the BBB (Rasband, 2016; Rowitch \& Kriegstein, 2010; Ullian et al., 2001). In disease states, astrocytes respond by expressing extracellular matrix molecules to form a glial scar that helps limit tissue damage but, unfortunately, prevents axonal regeneration (Rowitch \& Kriegstein, 2010; Sharma et al., 
2012; Sofroniew, 2015). The other macroglial cell population, oligodendrocytes, are derived from precursors in the ventral neural tube and subventricular zone (Rowitch, 2004; Rowitch \& Kriegstein, 2010). Following a series of differentiation steps, oligodendrocytes associate with and wrap axons with their multi-lamellar membrane. Oligodendrocyte - axonal interactions are also important for organizing axons, i.e., help with proper localization of sodium and potassium channels to promote rapid and efficient action potential propagation (Rowitch \& Kriegstein, 2010). Lastly, microglial cells are the innate immune cells of the CNS and are involved in pathogen removal and debris clearance (Domingues et al., 2016; Rasband, 2016). During development, microglial activation has been shown to secrete growth factors, prune synapses and remove dying cells (Peferoen et al., 2014; Rasband, 2016; Schafer et al., 2012; Wake et al., 2012).

Interestingly, oligodendrocytes demonstrate morphological, molecular and developmental origin heterogeneity (Hardy et al., 1996; Mayoral \& Chan, 2016; Ornelas et al., 2016; Tomassy et al., 2016; Zeisel et al., 2015). A recent study demonstrated that within the mouse cortex and hippocampus, single-cell RNA-seq revealed six subpopulations of oligodendrocytes and two subpopulations of astrocytes (Zeisel et al., 2015). Additionally, oligodendrocytes isolated from 10 regions within the CNS of juvenile and adult mice identified 13 distinct populations (Marques et al., 2016), further supporting the diversity amongst CNS myelinating glial cells.

Taken together, the diversity that exists within the nervous system ultimately contributes to the complexity with studying this intricate system. However, a comprehensive analysis 
of the genetic and molecular profile of glial cells and their subtypes is needed to develop a better understanding about mechanisms required for development, maintenance, injury responses and disease. Having a more comprehensive understanding about glial heterogeneity may also reveal novel therapeutic targets for treating neurodegenerative diseases.

\section{Do ectopic oligodendrocytes maintain their CNS profile and characteristics?}

In addition to glial cell heterogeneity, another interesting question that arises from this dissertation and that requires further investigation involves whether ectopic oligodendrocytes maintain their intrinsic properties, including responses to extrinsic cues, in their non-native environment of the PNS. As described in this dissertation and in a recent publication, ectopic oligodendrocyte progenitor cells migrate from the CNS to associate with and ensheath $e r b b 3 b^{-/}$zebrafish spinal motor axons (Smith et al., 2014). The presence of ectopic OPCs has also been reported within peripheral myelin mutants in several model systems, including zebrafish (Kucenas et al., 2009; Kucenas et al., 2008b), mouse (Coulpier et al., 2010; Weinberg et al., 1975) and humans (Coulpier et al., 2010). However, it is unknown whether the ectopic OPCs behave similarly in the PNS as within their native environment, the CNS.

During development, OPCs, derived from the ventral spinal cord and subventricular zone (Rowitch, 2004; Rowitch \& Kriegstein, 2010), proliferate and migrate to populate the CNS (Mayoral \& Chan, 2016). OPC differentiation occurs throughout the CNS, yet is spatiotemporally restricted as OPCs demonstrate contact-mediated repulsion behaviors 
when populating the CNS (Hughes et al., 2013; Kirby et al., 2006; Mayoral \& Chan, 2016). As oligodendrocytes differentiate and associate with axons, their multi-lamellar membrane becomes concentrically wrapped around axons, and one oligodendrocyte can myelinate up to 40 - 50 axons (McTigue \& Tripathi, 2008; Peferoen et al., 2014; Quarles et al., 2006), in contrast to the 1:1 relationship that PNS Schwann cells form with axons (Jessen \& Mirsky, 1991; 1997; 1998; 1999; 2010). Additionally, axonal activity has been shown to promote CNS myelination as inhibiting synaptic vesicle release results in myelination deficits, and promoting neuronal activity increases myelination processes (Mensch et al., 2015). Intrinsic differences are also involved with OPC differentiation, as OPCs from gray matter only differentiated once being transplanted into white matter (Mayoral \& Chan, 2016; Viganò et al., 2013) and white matter OPCs are more responsive to growth factors, such as platelet-derived growth factor (PDGF) (Hill et al., 2013; Mayoral \& Chan, 2016). In addition to intrinsic cues promoting OPC differentiation, extrinsic cues from the CNS environment can alter OPC behaviors. Over-expressing the growth factor PDGF results in more OPCs and accelerates OPC differentiation kinetics (Calver et al., 1998). Considering these inherent properties and responses by OPCs in the CNS, is this maintained in the PNS?

In vivo, time-lapse imaging of $e r b b 3 b^{-/}$zebrafish larvae demonstrates that multiple OPCs can migrate from the same MEP TZ, supporting two alternative hypotheses. First, is contact-mediated repulsion specific to the CNS environment, such that this behavior becomes irrelevant once the OPCs exit to associate with peripheral axons? Alternatively, are OPCs specified prior to exiting the pMN domain into the periphery and, because of 
this, no longer require contact mediate repulsion? Future studies investigating the specification timing of these ectopic OPCs in $e r b b 3 b^{-/}$will be beneficial for addressing whether contact-mediated repulsion is compromised in the ectopic OPCs, or if the OPCs have differentiated prior to exiting and no longer rely upon contact mediated repulsion.

Immunohistochemistry data using an antibody to Myelin Basic Protein (MBP) demonstrates MBP labeling along wildtype (Figure 6-1A) and $e r b b 3 b^{-/}$spinal motor axons (Figure 6-1B). However, the MBP labeling along mutant axons (Figure 6-1B) appears reminiscent of an oligodendrocyte myelinating multiple axons, as opposed to a single $\mathrm{MBP}^{+}$tube in wildtype (Figure 6-1A), reminiscent of Schwann cells forming a 1:1 relationship with axons. Ultrastructural analysis also reveals different myelinating glial observations. For example, there are myelinated peripheral axons in which the myelinating glial cell forms a 1:1 relationship with a peripheral axon, reminiscent of a Schwann cell. However, ultrastructural analysis also reveals the presence of multipolar cells, reminiscent of an OPC or oligodendrocyte. Do OPCs maintain their one to many ratios for every motor nerve? Or do the OPCs become a hybrid myelinating glial cell with both Schwann cell and oligodendrocyte characteristics? Another intriguing possibility is that CNS-derived Schwann cells may form and exit the CNS at time points that are not conducive for in vivo imaging assays, but prior to our ultrastructural TEM assessments in the adults. CNS-derived Schwann cells have been shown to arise from a common CNS progenitor in vitro (Mujtaba, 1998) as well as to remyelinate CNS axons following certain disease states (Akiyama et al., 2001; Blakemore, 2005; Crang et al., 2004; 


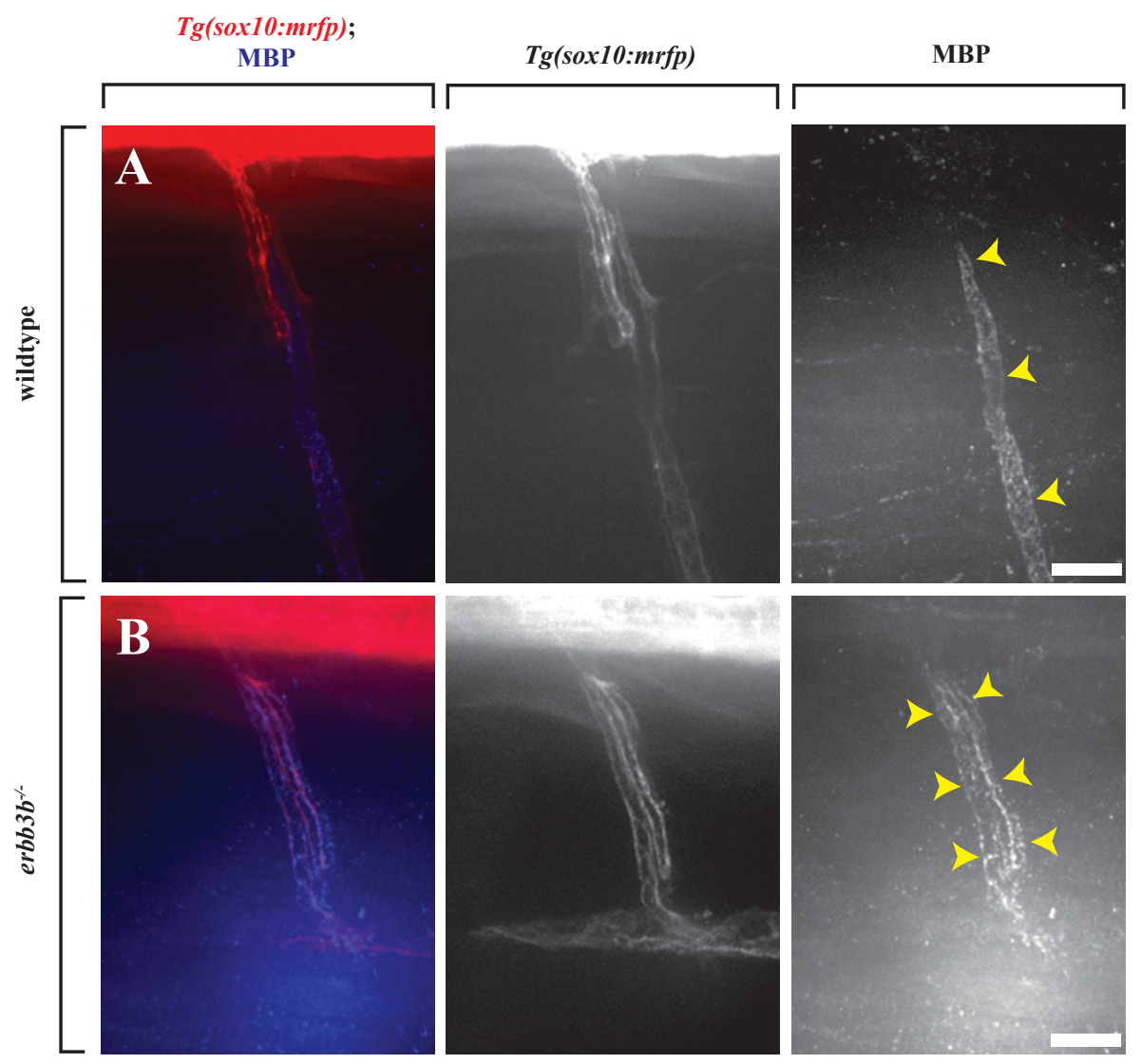

Figure 6-1. Myelin Basic Protein (MBP) labeling along wildtype and $e r b b 3 b^{-/}$motor axons. Images are lateral views of spinal cord with anterior to left and dorsal to top. (A - B) Immunohistochemistry in wildtype (A) and erbb3b $b^{\text {st48-- }}$ (B) zebrafish reveals MBP labeling (blue, arrowhead) by sox 10: $m r f p^{+}$(red) myelinating glial cells. Scale bars: $25 \mu \mathrm{m}$. 
Zawadzka et al., 2010). Thus, future investigations are needed to confirm the identity of the myelinating glial cells in the adult erbb3b $b^{-/}$TEM data.

Additionally, since electrical activity has been shown to promote OPC differentiation and myelination (Mensch et al., 2015), does blocking synaptic vesicle release using tetanus toxin prevent the ectopic OPCs from ensheathing peripheral axons? Since OPCs respond to extrinsic cues, like PDGF, does over-expressing PDGF in peripheral axons alter OPC behavior? Schwann cells have been shown to respond to the growth factor, Neuregulin-1 Type III (Nrg 1 Type III), that is expressed by axons (Michailov, 2004; Taveggia et al., 2005; 2010). Nrg 1 Type III binds to the ErbB2-ErbB3 heterocomplex on peripheral myelinating glial cells and, once this occurs, initiates the peripheral myelination signaling cascade (Brinkmann et al., 2008; Chen et al., 2006). In $\mathrm{nrg}^{226-/}$ embryos, peripheral myelinating glial cells fail to associate with motor axons, and in vivo imaging demonstrates ectopic exiting of OPCs from the CNS (Figure 6-2A). However, oligodendrocytes do not rely upon $\mathrm{Nrg} 1$ Type III expression to initiate differentiation and myelination of CNS axons (Brinkmann et al., 2008). Does this change when OPCs exit the CNS to associate with peripheral axons? Whether these OPCs associate with and myelinate the peripheral axons was not examined in $\mathrm{nrg}^{226-\digamma_{-}}$zebrafish. Future studies investigating the behavior of these ectopically located OPCs in the $\mathrm{nrg}^{226-/}$ mutant zebrafish will provide us with a better understanding as to whether this axonally-secreted growth factor remains dispensable for OPC differentiation and maturation when OPCs are within an ectopic environment, the PNS. Future studies should also investigate if other axonal-secreted growth factors, including PDGF, are upregulated following the 


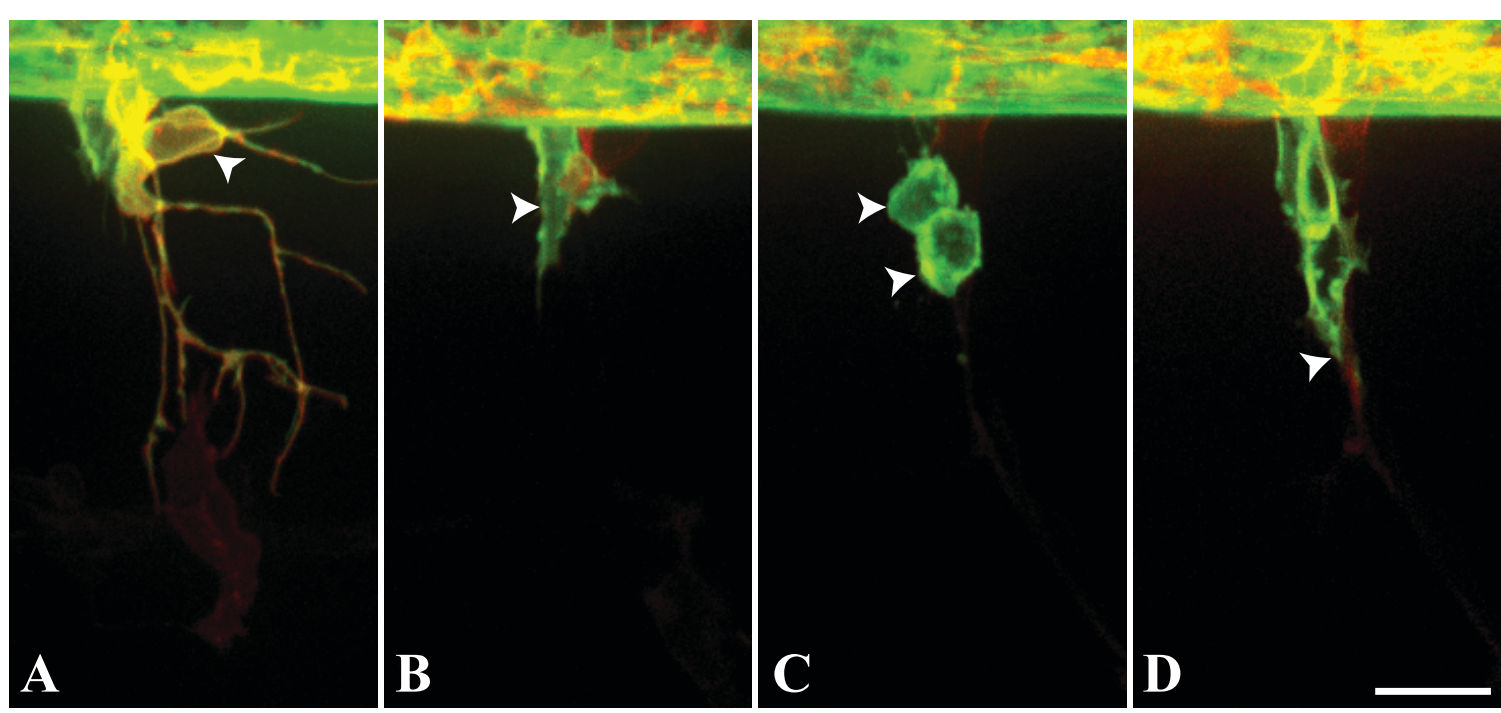

Figure 6-2. Glial cells associated with $\boldsymbol{n r g}^{\mathbf{z 2 6 - \digamma}}$ spinal motor axons. Images are lateral views of spinal cord with anterior to left and dorsal to top. (A) OPCs (arrowhead) ectopically exit the spinal cord in $\mathrm{nrg}^{226-1}$. (B - D) $n k x 2.2 a^{+}$perineurial glia (green, arrowhead) demonstrate stereotypic developmental behaviors in $n \mathrm{rg}^{226-1}$, including migration from the CNS (green, arrowhead) (B), proliferation (green, arrowhead) (C) and extension (green, arrowhead) (D). Scale bar: $25 \mu \mathrm{m}$. 
failure of peripheral glial cells from associating with the motor axons, and if overexpressing PDGF in erbb3b $3 b^{-/}$peripheral axons results in OPC proliferation and accelerated myelination.

Taken together, addressing whether some ectopic OPCs morph into having Schwann cell characteristics to adapt to the PNS environment accordingly, or if the ectopic OPCs maintain certain intrinsic behaviors that are characteristics of these glial cells in their resident environment, the CNS, may reveal important mechanisms required for promoting OPC health. This is important for the success of the proposed therapeutic strategy of recruiting OPCs out of their native CNS environment to fix or repair peripheral myelinopathies.

\section{The perineurium: an understudied structure and glial cell}

As described above, one main reason contributing to why perineurial cells have been largely understudied is due to a lack of markers that specifically label this cell population. Because of this, the majority of studies including data on the perineurium have reported ultrastructural alterations using electron microscopy. It wasn't until the pivotal work by Kucenas et al. (2008) that a new marker was identified that labels perineurial cells. The $n k x 2.2 a: m e g f p$ transgenic zebrafish line revealed that perineurial glial cells express $n k x 2.2 a$ (Kucenas et al., 2008b). However, $n k x 2.2 a$ is not specific to perineurial cells, and cells of the ventral floor plate, including OPCs, especially those of the myelinating oligodendrocyte fate (Kucenas et al., 2008a), also express $n k x 2.2 a$ (Kitada \& Rowitch, 2006; Kucenas et al., 2008a; Kuhlmann et al., 2008; Liu et al., 2007; Qi et al., 2001). 
Furthermore, in mouse, only a subpopulation of perineurial cells express $N k x 2.2 a$ (Clark et al., 2014).

Future studies are needed focusing specifically on identifying markers, preferably specific, for perineurial glial cells. One method for doing this would be to dissect the perineurium from peripheral nerves and perform RNA-seq on the dissected sample. To investigate for other markers in zebrafish, the motor nerves could be dissected from the spinal cord of adult zebrafish, $n k x 2.2 a^{+}$cells sorted using Fluorescence-activated cell sorting (FACS) and RNA-seq performed on these $n k x 2.2 a^{+}$cells. The RNA-seq data could be compared to peripheral myelinating glial transcriptomics data to identify markers specific within the peripheral $n k x 2.2 a^{+}$dataset. After revealing novel markers from the dataset, transgenic zebrafish lines could be created to confirm expression by perineurial glial cells. For markers expressed by perineurial glia, targeted genome editing approaches, including clustered, regularly interspaced, short palindromic repeat (CRISPR) with Cas9 RNA-guided nuclease, could be used to investigate the importance of these newly identified markers in perineurial glial, and overall nerve, development.

\section{Identifying mechanisms involved in perineurial developmental, differentiation and injury response behaviors}

Perineurial glial cells demonstrate relatively stereotypic behaviors during development, including migration from the CNS, proliferation and extension / retraction until ensheathment of motor axons and their associated myelinating glia is achieved. However, there is a lack of understanding regarding the mechanisms involved with each of these 
steps or behaviors. What is recruiting perineurial glial cells out of the CNS? Are perineurial - axonal interactions imperative for early perineurial migration? Or are neural crest cells responsible for signaling to perineurial cells to migrate into the periphery?

Interestingly, perineurial glia exit even though peripheral myelinating glial cells are absent in erbb3b $b^{-/}$, suggesting that it is not the interaction with Schwann cells or MEP glia that are needed for migration. However, do perineurial glia express erbb3b and, if so, is it the perturbation in erbb3b or the presence of ectopic OPCs that is responsible for the results presented in this dissertation. To address this, we used the $n r g^{z 26-/}$ zebrafish line (Perlin et al., 2011), described above, in which peripheral myelinating glial cells fail to associate with motor axons, resulting in the ectopic exiting of OPCs from the CNS (Figure 6-2A). Since erbb3b is intact in these larvae, we assayed for perineurial developmental behaviors in $n r g^{z 26-/-}$ embryos and observed migration from the CNS (Figure 6-2B), proliferation (Figure 6-2C) and extension (Figure 6-2D). However, future studies, such as double fluorescent in situ hybridization including an $n k x 2.2 a$ probe to label perineurial cells and an erbb3b probe to label the receptor to look for colocalization, are needed to definitely demonstrate if perineurial cells express erbb3b. Additionally, more careful quantification of perineurial behaviors in $\mathrm{nrg}^{z 26-/}$ zebrafish are needed to confirm the finding that perineurial glial behaviors observed via in vivo timelapse imaging are comparable to $e r b b 3 b^{-/}$and wildtype perineurial behaviors.

Furthermore, assaying perineurial glial migration using $e r b b 3 b^{-/}$spinal motor nerves does not rule out the possibility that early neural crest cells are communicating with 
perineurial cells, as neural crest cells migrate in $e r b b 3 b^{-/-}$mutants, but fail to associate with axons to differentiate into Schwann cells. In regards to perineurial - axonal interactions, electrical activity has not been investigated as a potential mechanism promoting early perineurial migration. Since perineurial cells do not exit in zebrafish harboring mutations in mont-blanc (mob) and mother-superior (mos) (mob;mos), which are deficient for the transcription factors tfap $2 a$ and foxd3, respectively, and lack all neural crest derivatives including Schwann cells, it is possible that perineurial cells either require neural crest cells or rely upon tfap $2 a$ or foxd3. Laser ablation of neural crest cells before their migration, coupled with in vivo imaging to determine if perineurial cells migrate from the CNS or not, will be useful for addressing whether perineurial cells require early interactions with neural crest cells to exit into the periphery. Additionally, using either mont-blanc or mother-superior zebrafish, and not the double mutant $m o b ; m o s$, and investigating for perineurial migration would allow us to rule out if tfap $2 a$ or foxd3 are needed. To address whether axonal electrical activity is required for perineurial migration, zebrafish embryos can be treated with tetanus toxin to block synaptic vesicle release, and perineurial developmental behaviors, including migration from the CNS, can be investigated.

In contrast to the early developmental processes of migration, proliferation and extension/ retraction, normal perineurial morphology and differentiation rely upon interactions with Schwann cells as these behaviors are compromised in erbb3b $b^{-/}$zebrafish. However, signaling mechanisms responsible for perineurial differentiation are not well understood. Based on ultrastructural data in mice harboring mutations in Schwann cell-derived 
factors, such as desert hedgehog (Dhh), Sox10 and the G protein-coupled receptor GPR126, perineurial morphology is significantly altered (Finzsch et al., 2010; Mirsky et al., 1999; Monk et al., 2011; Parmantier et al., 1999). The perineurium appears thin, disorganized and wavy, and compartmentalizes the nerve into smaller compartments called mini-fascicles. Perineurial barrier function was also compromised in $D h h^{-/}$mice (Parmantier et al., 1999). This alteration in perineurium morphology in Dhh, Sox10 and GPR126 mutant mice has been proposed to be caused by the lack of Schwann cellderived Dhh signaling (Finzsch et al., 2010; Monk et al., 2011; Parmantier et al., 1999). Perineurial cells express the Dhh receptor Ptc (Mirsky et al., 1999; Parmantier et al., 1999), Sox10 has been shown to activate Dhh signaling (Kuspert et al., 2012) and GPR126 mutant mice lack Dhh signaling in their peripheral nerves (Monk et al., 2011). Thus, the Dhh - Ptc signaling mechanism is important in promoting proper perineurial morphology and differentiation. Future studies are needed to investigate the expression of Dhh and Ptc along erbb3b $3 b^{-\alpha}$ motor nerves. However, because peripheral myelinating glial cells are absent along spinal motor nerves in these mutants, I would expect there to be a lack of Dhh - Ptc signaling. A lack of Dhh - Ptc signaling may explain the morphology and differentiation defects, including the loss of perineurial barrier integrity as evidenced by infiltration of a fluorescent tracer dye, we observed along erbb $3 b^{-/}$motor nerves.

Although Dhh - Ptc signaling has been shown to be important in perineurial differentiation in mice, our results suggest this is not the only signaling mechanism promoting perineurial differentiation. We revealed that transforming growth factor $\beta 1$ promotes perineurial maturation by influencing the expression of tight junctional proteins 
like zonula occludens-1 (ZO-1). We identified that phosphorylated Smad3 is present along peripheral nerves in both wildtype and $e r b b 3 b^{-/-}$larvae and, since pSmad3 is a downstream signaling transducer of TGF- $\beta 1$ (Kitisin et al., 2007), suggests that TGF- $\beta 1$ signaling is present along these peripheral nerves. Following inhibition of TGF- $\beta 1$ using the small molecule inhibitor SB431542, we observed a significant decrease in tight junctional protein ZO-1 labeling in both wildtype and mutant larvae treated with the inhibitor as compared to larvae treated with DMSO control, suggesting that TGF- $\beta 1$ is involved in perineurial glial differentiation. OPCs have been shown to promote BBB integrity and the expression of ZO-1 within the BBB via TGF- $\beta 1$ signaling (Seo et al., 2014). Interestingly, Schwann cells also express TGF- $\beta 1$ during development (Feng \& Ko, 2008; Scherer et al., 1993). Future studies are needed to investigate whether perineurial cells express TGF- $\beta 1$ receptors, since myelinating glial cells appear to be a likely source of the TGF- $\beta 1$ ligand. Double fluorescent in situ hybridization using an $n k x 2.2 a$ probe to label perineurial cells and $\operatorname{tg} f-\beta R I$ or $\operatorname{tg} f-\beta R I I$ to label TGF- $\beta 1$ receptors will be useful for identifying if perineurial cells express these receptors in zebrafish. To investigate whether the perineurium express TGF- $\beta$ receptors in mouse, immunohistochemistry using an antibody to anti-TGF- $\beta$ Receptor I (rabbit) and an antibody to 8.1.1 (podoplanin; mouse) to label the perineurium can be performed on cross-sections of motor nerves to assay for co-localization. It would also be interesting to investigate if perineurial maintenance relies upon TGF- $\beta$ signaling by administering the small molecule inhibitor followed by assaying for ZO-1 expression and comparing inhibitor treated to control treated animals. This assay could be performed in either zebrafish or mouse. 
As previously described, perineurial cells have been shown to be essential mediators involved in injury responses following motor axonal transection (Lewis \& Kucenas, 2013; 2014). Unfortunately, potential signaling mechanisms involved in promoting perineurial injury responses are unknown. Although the work by Lewis et al. (2014) characterized at least three signaling interactions post-injury, signaling mechanisms mediating the interactions were not revealed. Certain injury response behaviors, i.e., membrane extension towards the transection site, may be mediated by axonal cues and/or peripheral myelinating glial cues. Since perineurial glial cells do not respond to muscle injuries (Lewis \& Kucenas, 2014), axonal cues may be responsible for alerting the perineurium to an injury. However, perineurial glia fail to extend membrane beyond the injury site following transection of axonal tracts containing ectopic oligodendrocytes, demonstrating perineurial - peripheral myelinating glial interactions are important for recruiting perineurial membrane to the transection site. Lastly, perineurial - perineurial interactions are essential for the formation of a glial bridge that spans the transection site (Lewis \& Kucenas, 2014). Future studies focusing on identifying signaling mechanisms specific for each perineurial response behavior are imperative for potentially revealing novel therapeutic strategies for promoting efficient and effective regeneration. One approach for identifying therapeutic targets that promote regeneration is to perform a pharmaceutical library drug screen in zebrafish following axonal transection, and investigate perineurial injury responses using in vivo imaging. This may reveal drugs that promote efficient repair and that may be useful therapies for patients recovering from peripheral axonal transections. 


\section{Oligodendrocyte cytoskeletal responses following a demyelinating insult}

Multiple sclerosis is a neurodegenerative disorder in which the myelin sheath and oligodendrocytes are attacked. There is remarkable heterogeneity amongst MS patients, ranging from clinical symptoms, histopathological lesions and therapy responses (Lassmann et al., 2001; Lucchinetti et al., 2000). In addition to the vast heterogeneity, the primary etiology of MS remains unknown, resulting in the proposal of two models: "outside-in" and "inside-out" (Stys et al., 2012). The outside-in model suggests that the primary hit, or initial trigger, occurs within the periphery in which the immune system becomes primed and T-cells become autoreactive against myelin antigens, infiltrating into the CNS and destroying myelin and oligodendrocytes (Frohman et al., 2006; Lucchinetti et al., 2000; Stys et al., 2012). In contrast, the inside-out model proposes that the primary insult is caused by an oligodendrogliopathy or myelin defect, resulting in the priming of the immune system and the subsequent neuroinflammatory response (Barnett \& Prineas, 2004; Lucchinetti et al., 2000; Stys et al., 2012). Because the primary etiology is unknown, and mammalian models, including human pathology samples, do not allow for investigating myelin breakdown or repair in real time, there are unappreciated mechanics underlying demyelination. Having various animal models, including capitalizing on the in vivo imaging capabilities of zebrafish, is essential for developing a better understanding about processes involved in myelin breakdown and oligodendrocyte response to a demyelinating insult.

Using the novel focal demyelination model in zebrafish, described in Chapter 5 of this dissertation, we visualized myelin membrane changes following injection of a 
demyelinating drug called lysolecithin. Interestingly, myelin membrane changes, such as ovoids or intramyelinic edema, have been described based on ultrastructural data (Acar et al., 2004; Kaufmann et al., 2012; McMartin et al., 1997; Prineas \& Connell, 1979; Triarhou \& Herndon, 1985), and are a hallmark of myelin degradation. This edema fluid accumulation has been proposed to cause the myelin sheath to separate along the intraperiod line (Hirano et al., 1965; Hirano \& Llena, 2006; Kaufmann et al., 2012; McMartin et al., 1997). Interestingly, following injection of lysolecithin into the rat spinal cord, the presence of these vacuoles have been reported based on ultrastructural EM data (Triarhou \& Herndon, 1985). To our knowledge, this is the first report visualizing the formation of these myelin ovoids or intramyelinic vacuoles following a demyelinating insult. However, future studies, including ultrastructural EM data, are needed to confirm that these ovoids are intramyelinic edema vacuoles.

Additionally, the actin cytoskeleton is involved with the formation of onion bulbs following in vitro peripheral nerve injuries as inhibiting actin polymerization prevented the formation of ovoids, or these onion bulb structures (Jung et al., 2011). Since actin dynamics are essential mediators of developmental myelination (Nawaz et al., 2015; Zuchero et al., 2015), and are also involved in myelin membrane fragmentation following peripheral nerve injuries (Jung et al., 2011), it is reasonable to hypothesize that the dynamic rearrangement of filamentous actin within the oligodendrocyte cytoskeleton may actively mediate the myelin membrane changes we observed following a demyelinating insult. Future studies are needed to investigate whether actin dynamics are actively changing the oligodendrocyte cytoskeleton to form myelin ovoids in response to 
a demyelinating insult, or to determine if filamentous actin rearrangement is responding passively to myelin membrane changes. One assay involves treating zebrafish with an actin polymerization inhibitor, such as latrunculin A. The larvae can be pre-treated before demyelination injections by submerging in the drug solution, or the latrunculin A can be included within the lysolecithin injection cocktail and distributed focally within the spinal cord, presumably accessing the same regions as the lysolecithin dispersal. In vivo timelapse imaging will then be performed and the number of $m b p^{+}$ovoids will be quantified. If actin polymerization is actively responsible for forming the ovoids we observe postinjection with lysolecithin, inhibiting this process should result in a reduction of, or no, ovoids forming. However, if actin arrangement is not actively involved in forming the myelin ovoids, we should expect to see myelin membrane changes and ovoid formation within the latrunculin A treated zebrafish following exposure to lysolecithin. Thus, the role of the oligodendrocyte cytoskeleton, including the temporal dynamics of actin rearrangement and myelin breakdown following a demyelinating insult, requires further elucidation to develop a better understanding about the mechanics involved.

\section{Conclusion}

Taken together, findings from this dissertation further demonstrate the importance of glial-glial interactions and glial responses in the nervous system. Although additional studies are needed to identify specific markers for the various glial subtypes, as well as revealing mechanisms that mediate glial behaviors, interactions and responses, overall, this work provides a better understanding and appreciation about the plasticity of glial cells both during development and in response to myelinopathies. 


\title{
Appendix: Abbreviations
}

\begin{abstract}
Abbreviations are listed in alphabetical order. For transgene abbreviations and mutant lines, refer to Table $2-1$ on page 34 .
\end{abstract}

\begin{tabular}{|c|c|}
\hline $\mathrm{BBB}$ & blood-brain barrier \\
\hline $\mathrm{BNB}$ & blood-nerve barrier \\
\hline $\mathrm{CNS}$ & central nervous system \\
\hline Dhh & desert hedgehog \\
\hline dpf & days post fertilization \\
\hline EAE & experimental autoimmune encephalomyelitis \\
\hline EM & electron microscopy \\
\hline Erbb3 & receptor tyrosine kinase erbB-3 \\
\hline GFP & green fluorescent protein \\
\hline GPR126 & G protein-coupled receptor 126 \\
\hline $\mathrm{H} \& \mathrm{E}$ & Hematoxylin and Eosin \\
\hline hpf & hours post fertilization \\
\hline hpi & hours post injection \\
\hline hpt & hours post transection \\
\hline $\mathrm{IHC}$ & immunohistochemistry \\
\hline MEP & motor exit point \\
\hline MEP TZ & motor exit point transition zone \\
\hline
\end{tabular}




$\begin{array}{ll}\text { Nrg1 } & \text { Neuregulin 1 Type III } \\ \text { OPC } & \text { oligodendrocyte progenitor cell } \\ \text { pLLn } & \text { posterior lateral line nerve } \\ \text { pMN } & \text { motor neuron progenitor domain } \\ \text { PNS } & \text { peripheral nervous system } \\ \text { Ptc } & \text { patched } \\ \text { RFP } & \text { red fluorescent protein } \\ \text { SCP } & \text { Schwann cell precursor } \\ \text { Sox10 } & \text { SRY (sex-determining region Y)-box } 10 \\ \text { TEM } & \text { transmission electron microscopy } \\ \text { TGF- } \beta 1 & \text { transforming growth factor beta-1 } \\ \text { ZO-1 } & \text { zonula occludens-1 }\end{array}$




\section{References}

Acar, G., Tanriover, G., Demir, N., Kayisli, U. A., Leyla Sati, G., Yaba, A., Idiman, E., \& Demir, R. (2004). Ultrastructural and immunohistochemical similarities of two distinct entities; multiple sclerosis and hereditary motor sensory neuropathy. Acta Histochemica, 106(5), 363-371. http://doi.org/10.1016/j.acthis.2004.08.004

Akert, K., Sandri, C., Weibel, E. R., Peper, K., \& Moor, H. (1976). The fine structure of the perineural endothelium. Cell and Tissue Research, 165(3), 281-295.

Akiyama, Y., Honmou, O., Kato, T., Uede, T., Hashi, K., \& Kocsis, J. D. (2001). Transplantation of Clonal Neural Precursor Cells Derived from Adult Human Brain Establishes Functional Peripheral Myelin in the Rat Spinal Cord. Experimental Neurology, 167(1), 27-39. http://doi.org/10.1006/exnr.2000.7539

Allodi, I., Udina, E., \& Navarro, X. (2012). Specificity of peripheral nerve regeneration: Interactions at the axon level. Progress in Neurobiology, 98(1), 16-37. http://doi.org/10.1016/j.pneurobio.2012.05.005

Allt, G. G., \& Lawrenson, J. G. J. (2000). The blood-nerve barrier: enzymes, transporters and receptors--a comparison with the blood-brain barrier. Brain Research Bulletin, $52(1), 1-12$. 
Almeida, R. G., Czopka, T., ffrench-Constant, C., \& Lyons, D. A. (2011). Individual axons regulate the myelinating potential of single oligodendrocytes in vivo. Development, 138(20), 4443-4450. http://doi.org/10.1242/dev.071001

Appel, B., Korzh, V., Glasgow, E., Thor, S., Edlund, T., Dawid, I. B., \& Eisen, J. S. (1995). Motoneuron fate specification revealed by patterned LIM homeobox gene expression in embryonic zebrafish. Development, 121(12), 4117-4125.

Arduini, B. L., Bosse, K. M., \& Henion, P. D. (2009). Genetic ablation of neural crest cell diversification. Development, 136(12), 1987-1994. http://doi.org/10.1242/dev.033209

Arnett, H. A., Fancy, S. P. J., Alberta, J. A., Zhao, C., Plant, S. R., Kaing, S., Raine, C. S., Rowitch, D. H., Franklin, R. J. M., \& Stiles, C. D. (2004). bHLH transcription factor Olig1 is required to repair demyelinated lesions in the CNS. Science, 306(5704), 2111-2115. http://doi.org/10.1126/science.1103709

Arnett, H. A., Mason, J., Marino, M., Suzuki, K., Matsushima, G. K., \& Ting, J. P. (2001). TNF alpha promotes proliferation of oligodendrocyte progenitors and remyelination. Nature Neuroscience, 4(11), 1116-1122. http://doi.org/10.1038/nn738

Arthur-Farraj, P. J., Latouche, M., Wilton, D. K., Quintes, S., Chabrol, E., Banerjee, A., et al. (2012). c-Jun reprograms Schwann cells of injured nerves to generate a repair 
cell essential for regeneration. Neuron, 75(4), 633-647. http://doi.org/10.1016/j.neuron.2012.06.021

Auld, V. (1999). Glia as mediators of growth cone guidance: studies from insect nervous systems. Cellular and Molecular Life Sciences, 55(11), 1377-1385.

Azin, M., Mirnajafi-Zadeh, J., \& Javan, M. (2015). Fibroblast Growth Factor-2 Enhanced The Recruitment of Progenitor Cells and Myelin Repair in Experimental Demyelination of Rat Hippocampal Formations. Cell Journal, 17(3), 540-456.

Baloh, R. H., Tansey, M. G., Lampe, P. A., Fahrner, T. J., Enomoto, H., Simburger, K. S., Leitner, M. L., Araki, T., Johnson, E., \& Milbrandt, J. (1998). Artemin, a novel member of the GDNF ligand family, supports peripheral and central neurons and signals through the GFRalpha3-RET receptor complex. Neuron, 21(6), 1291-1302.

Banerjee, S., Isaacman-Beck, J., Schneider, V. A., \& Granato, M. (2013). A Novel Role for Lh3 Dependent ECM Modifications during Neural Crest Cell Migration in $\begin{array}{llll}\text { Zebrafish. } & \text { PLoS } & \text { ONE } & \text { e54609. }\end{array}$ http://doi.org/10.1371/journal.pone.0054609.g004

Barnett, M. H., \& Prineas, J. W. (2004). Relapsing and remitting multiple sclerosis: Pathology of the newly forming lesion. Annals of Neurology, 55(4), 458-468. http://doi.org/10.1002/ana.20016 
Barrallo-Gimeno, A., Holzschuh, J., Driever, W., \& Knapik, E. W. (2004). Neural crest survival and differentiation in zebrafish depends on mont blanc/tfap2a gene function. Development, 131(7), 1463-1477. http://doi.org/10.1242/dev.01033

Baumann, N. N., \& Pham-Dinh, D. D. (2001). Biology of oligodendrocyte and myelin in the mammalian central nervous system. Physiological Reviews, 81(2), 871-927.

Binari, L. A., Lewis, G. M., \& Kucenas, S. (2013). Perineurial glia require Notch signaling during motor nerve development but not regeneration. Journal of Neuroscience, 33(10), 4241-4252. http://doi.org/10.1523/JNEUROSCI.489312.2013

Birchmeier, C. (2009). ErbB receptors and the development of the nervous system. Experimental Cell Research, 315(4), 611-618. http://doi.org/10.1016/j.yexcr.2008.10.035

Bitsch, A., Kuhlmann, T., Da Costa, C., Bunkowski, S., Polak, T., \& Bruck, W. (2000). Tumour necrosis factor alpha mRNA expression in early multiple sclerosis lesions: correlation with demyelinating activity and oligodendrocyte pathology. Glia, 29(4), 366-375.

Blakemore, W. F. (1973). Demyelination of the superior cerebellar peduncle in the mouse induced by cuprizone. Journal of the Neurological Sciences, 20(1), 63-72. 
Blakemore, W. F. (1974). Remyelination of the superior cerebellar peduncle in old mice following demyelination induced by cuprizone. Journal of the Neurological Sciences, 22(1), 121-126.

Blakemore, W. F. (2005). The case for a central nervous system (CNS) origin for the Schwann cells that remyelinate CNS axons following concurrent loss of oligodendrocytes and astrocytes. Neuropathology and Applied Neurobiology, 31(1), 1-10. http://doi.org/10.1038/nrn721

Blakemore, W. F., \& Franklin, R. J. M. (2008). Remyelination in experimental models of toxin-induced demyelination. Current Topics in Microbiology and Immunology, 318, $193-212$.

Blakemore, W. F., Eames, R. A., Smith, K. J., \& McDonald, W. I. (1977). Remyelination in the spinal cord of the cat following intraspinal injections of lysolecithin. Journal of the Neurological Sciences, 33(1-2), 31-43.

Bourne, G. H. (1968). The Structure and Function of Nervous Tissue Vol 5 (Vol. 1, pp. 379-459). New York: Academic Press.

Brinkmann, B. G., Agarwal, A., Sereda, M. W., Garratt, A. N., Müller, T., Wende, H., et al. (2008). Neuregulin-1/ErbB Signaling Serves Distinct Functions in Myelination of the Peripheral and Central Nervous System. Neuron, 59(4), 581-595. 
Britsch, S. (2001). The transcription factor Sox10 is a key regulator of peripheral glial development. Genes \& Development, 15(1), 66-78. http://doi.org/10.1101/gad.186601

Brushart, T. M., Aspalter, M., Griffin, J. W., Redett, R., Hameed, H., Zhou, C., Wright, M., Vyas, A., \& Höke, A. (2013). Schwann cell phenotype is regulated by axon modality and central-peripheral location, and persists in vitro. Experimental Neurology, 247, 272-281. http://doi.org/10.1016/j.expneurol.2013.05.007

Brushart, T. M., Henry, E. W., \& Mesulam, M. M. (1981). Reorganization of muscle afferent projections accompanies peripheral nerve regeneration. Neuroscience, $6(10)$, 2053-2061.

Bunge, M. B., Wood, P. M., Tynan, L. B., Bates, M. L., \& Sanes, J. R. (1989). Perineurium originates from fibroblasts: demonstration in vitro with a retroviral marker. Science, 243(4888), 229-231.

Busch, S. A., \& Silver, J. (2007). The role of extracellular matrix in CNS regeneration. Current Opinion in Neurobiology, 17(1), 120-127. http://doi.org/10.1016/j.conb.2006.09.004

Buttermore, E. D., Thaxton, C. L., \& Bhat, M. A. (2013). Organization and maintenance of molecular domains in myelinated axons. Journal of Neuroscience Research, 91(5), 603-622. http://doi.org/10.1002/jnr.23197 
Bø, L., Esiri, M., Evangelou, N., \& Kuhlmann, T. (2012). Demyelination and Remyelination in Multiple Sclerosis (pp. 23-45). Boston, MA: Springer US. http://doi.org/10.1007/978-1-4614-2218-1_2

Calver, A. R., Hall, A. C., Yu, W. P., Walsh, F. S., Heath, J. K., Betsholtz, C., \& Richardson, W. D. (1998). Oligodendrocyte population dynamics and the role of PDGF in vivo. Neuron, 20(5), 869-882.

Caroni, P., \& Schwab, M. E. (1988). Antibody against myelin-associated inhibitor of neurite growth neutralizes nonpermissive substrate properties of CNS white matter. Neuron, 1(1), 85-96.

Carvalho, A. T., \& Sá, M. J. (2012). Switching and Escalating Therapy in Long-Lasting Multiple Sclerosis: Not Always Necessary. ISRN Neurology, 2012(10), 1-5. http://doi.org/10.1111/j.1600-0404.2006.00637.x

Casari, A., Schiavone, M., Facchinello, N., Vettori, A., Meyer, D., Tiso, N., Moro, E., \& Argenton, F. (2014). Developmental Biology. Developmental Biology, 396(1), 8193. http://doi.org/10.1016/j.ydbio.2014.09.025

Chan, J., Aoki, C., \& Pickel, V. M. (1990). Optimization of differential immunogoldsilver and peroxidase labeling with maintenance of ultrastructure in brain sections before plastic embedding. Journal of Neuroscience Methods, 33(2-3), 113-127. 
Chen, S., Velardez, M. O., Warot, X., Yu, Z.-X., Miller, S. J., Cros, D., \& Corfas, G. (2006). Neuregulin 1-erbB signaling is necessary for normal myelination and sensory function. Journal of Neuroscience, 26(12), 3079-3086. http://doi.org/10.1523/JNEUROSCI.3785-05.2006

Chiang, C. S., Powell, H. C., Gold, L. H., Samimi, A., \& Campbell, I. L. (1996). Macrophage/microglial-mediated primary demyelination and motor disease induced by the central nervous system production of interleukin-3 in transgenic mice. Journal of Clinical Investigation, 97(6), 1512-1524. http://doi.org/10.1172/JCI118574

Chung, A.-Y., Kim, P.-S., Kim, S., Kim, E., Kim, D., Jeong, I., Kim, H.-K., Ryu, J.-H., Kim, C.-H., Choi, J., Seo, J.-H., \& Park, H.-C. (2013). Generation of demyelination models by targeted ablation of oligodendrocytes in the zebrafish CNS. Molecules and Cells, 36(1), 82-87. http://doi.org/10.1007/s10059-013-0087-9

Clark, J. K., O'keefe, A., Mastracci, T. L., Sussel, L., Matise, M. P., \& Kucenas, S. (2014). Mammalian Nkx2.2 +perineurial glia are essential for motor nerve development. Developmental Dynamics, 243(9), 1116-1129. http://doi.org/10.1002/dvdy.24158

Confavreux, C., Vukusic, S., Moreau, T., \& Adeleine, P. (2000). Relapses and progression of disability in multiple sclerosis. The New England Journal of Medicine, 343(20), 1430-1438. http://doi.org/10.1056/NEJM200011163432001 
Conley, F. K., Rubinstein, L. J., \& Spence, A. M. (1976). Studies on experimental malignant nerve sheath tumors maintained in tissue and organ culture systems. II. Electron microscopy observations. Acta Neuropathologica, 34(4), 293-310.

Copelman, C. A., Diemel, L. T., Gveric, D., Gregson, N. A., \& Cuzner, M. L. (2001). Myelin phagocytosis and remyelination of macrophage-enriched central nervous system aggregate cultures. Journal of Neuroscience Research, 66(6), 1173-1178. http://doi.org/10.1002/jnr.10026

Corfas, G., Velardez, M. O., Ko, C.-P., Ratner, N., \& Peles, E. (2004). Mechanisms and Roles of Axon-Schwann Cell Interactions. Journal of Neuroscience, 24(42), 92509260. http://doi.org/10.1523/JNEUROSCI.3649-04.2004

Corson, J., Nahmani, M., Lubarsky, K., Badr, N., Wright, C., \& Erisir, A. (2009). Sensory activity differentially modulates N-methyl-D-aspartate receptor subunits $2 \mathrm{~A}$ and 2B in cortical layers. Neuroscience, 163(3), 920-932. http://doi.org/10.1016/j.neuroscience.2009.07.016

Coulpier, F., Decker, L., Funalot, B., Vallat, J.-M., Garcia-Bragado, F., Charnay, P., \& Topilko, P. (2010). CNS/PNS boundary transgression by central glia in the absence of Schwann cells or Krox20/Egr2 function. Journal of Neuroscience, 30(17), 59585967. http://doi.org/10.1523/JNEUROSCI.0017-10.2010 
Crang, A. J., Gilson, J. M., Li, W. W., \& Blakemore, W. F. (2004). The remyelinating potential and in vitro differentiation of MOG-expressing oligodendrocyte precursors isolated from the adult rat CNS. European Journal of Neuroscience, 20(6), 14451460. http://doi.org/10.1038/35097593

Curado, S., Stainier, D. Y. R., \& Anderson, R. M. (2008). Nitroreductase-mediated cell/tissue ablation in zebrafish: a spatially and temporally controlled ablation method with applications in developmental and regeneration studies. Nature Protocols, 3(6), 948-954. http://doi.org/10.1038/nprot.2008.58

Czopka, T., \& Lyons, D. A. (2011). Dissecting Mechanisms of Myelinated Axon Formation Using Zebrafish. Methods in Cell Biology (Third Edition, Vol. 105, pp. 25-62). Elsevier Inc. http://doi.org/10.1016/B978-0-12-381320-6.00002-3

Czopka, T., ffrench-Constant, C., \& Lyons, D. A. (2013). Individual Oligodendrocytes Have Only a Few Hours in which to Generate New Myelin Sheaths In Vivo. Developmental Cell, 25(6), 599-609. http://doi.org/10.1016/j.devcel.2013.05.013

Dal Canto, M. C., Melvold, R. W., Kim, B. S., \& Miller, S. D. (1995). Two models of multiple sclerosis: experimental allergic encephalomyelitis (EAE) and Theiler's murine encephalomyelitis virus (TMEV) infection. A pathological and immunological comparison. Microscopy Research and Technique, 32(3), 215-229. http://doi.org/10.1002/jemt.1070320305 
Davison, J. M., Akitake, C. M., Goll, M. G., Rhee, J. M., Gosse, N., Baier, H., Halpern, M. E., Leach, S. D., \& Parsons, M. J. (2007). Transactivation from Gal4-VP16 transgenic insertions for tissue-specific cell labeling and ablation in zebrafish. Developmental Biology, 304(2), 811-824. http://doi.org/10.1016/j.ydbio.2007.01.033

Davison, S. P., McCaffrey, T. V., Porter, M. N., \& Manders, E. (1999). Improved nerve regeneration with neutralization of transforming growth factor-beta1. The Laryngoscope, 109(4), 631-635. http://doi.org/10.1097/00005537-199904000-00021

De Angelis, F., Bernardo, A., Magnaghi, V., Minghetti, L., \& Tata, A. M. (2012). Muscarinic receptor subtypes as potential targets to modulate oligodendrocyte progenitor survival, proliferation, and differentiation. Developmental Neurobiology, 72(5), 713-728. http://doi.org/10.1002/dneu.20976

Denic, A., Johnson, A. J., Bieber, A. J., Warrington, A. E., Rodriguez, M., \& Pirko, I. (2011). The relevance of animal models in multiple sclerosis research. Pathophysiology, 18(1), 21-29. http://doi.org/10.1016/j.pathophys.2010.04.004

Domingues, H. S., Portugal, C. C., Socodato, R., \& Relvas, J. B. (2016). Oligodendrocyte, Astrocyte, and Microglia Crosstalk in Myelin Development, Damage, and Repair. Frontiers in Cell and Developmental Biology, 4(268), 89. http://doi.org/10.1242/dev.129304 
Doring, A., Sloka, S., Lau, L., Mishra, M., van Minnen, J., Zhang, X., Kinniburgh, D., Rivest, S., \& Yong, V. W. (2015). Stimulation of Monocytes, Macrophages, and Microglia by Amphotericin B and Macrophage Colony-Stimulating Factor Promotes Remyelination. Journal of Neuroscience, 35(3), 1136-1148. http://doi.org/10.1523/JNEUROSCI.1797-14.2015

Dousset, V., Brochet, B., Vital, A., Gross, C., Benazzouz, A., Boullerne, A., Bidabe, A.M., Gin, A.-M., \& Caille, J.-M. (1995). Lysolecithin-induced demyelination in primates: preliminary in vivo study with $\mathrm{MR}$ and magnetization transfer. AJNR. American Journal of Neuroradiology, 16(2), 225-231.

Dutton, K. A., Pauliny, A., Lopes, S. S., Elworthy, S., Carney, T. J., Rauch, J., Geisler, R., Hafter, P., Kelsh, R. N. (2001). Zebrafish colourless encodes sox10 and specifies non-ectomesenchymal neural crest fates. Development, 128(21), 4113-4125.

Dyck, P. J., \& Thomas, P. K. (2005). Peripheral Neuropathy. (E. Saunders, Ed.) (4 ed.). Philadelphia.

Eisen, J. S. (1991). Developmental neurobiology of the zebrafish. Journal of Neuroscience, 11(2), 311-317.

Ek, C. J., Habgood, M. D., Dziegielewska, K. M., \& Saunders, N. R. (2003). Structural characteristics and barrier properties of the choroid plexuses in developing brain of 
the opossum (Monodelphis Domestica). The Journal of Comparative Neurology, 460(4), 451-464. http://doi.org/10.1002/cne.10661

Ellett, F., Pase, L., Hayman, J. W., Andrianopoulos, A., \& Lieschke, G. J. (2011). mpeg1 promoter transgenes direct macrophage-lineage expression in zebrafish. Blood, 117(4), e49-56. http://doi.org/10.1182/blood-2010-10-314120

Emery, B. (2010). Regulation of oligodendrocyte differentiation and myelination. Science, 330(6005), 779-782. http://doi.org/10.1126/science.1190927

Erisir, A., \& Dreusicke, M. (2005). Quantitative morphology and postsynaptic targets of thalamocortical axons in critical period and adult ferret visual cortex. The Journal of Comparative Neurology, 485(1), 11-31. http://doi.org/10.1002/cne.20507

Fang, Y., Lei, X., Li, X., Chen, Y., Xu, F., Feng, X., Wei, S., \& Li, Y. (2015). A novel model of demyelination and remyelination in a GFP-transgenic zebrafish. Biology Open, 4(1), 62-68. http://doi.org/10.1242/bio.201410736

Faroni, A., Mobasseri, S. A., Kingham, P. J., \& Reid, A. J. (2015). Peripheral nerve regeneration: experimental strategies and future perspectives. Advanced Drug Delivery Reviews, 82-83, 160-167. http://doi.org/10.1016/j.addr.2014.11.010 
Fawcett, J. W. (2006). Overcoming inhibition in the damaged spinal cord. Journal of Neurotrauma, 23(3-4), 371-383. http://doi.org/10.1089/neu.2006.23.371

Feng, Z., \& Ko, C. P. (2008). Schwann Cells Promote Synaptogenesis at the Neuromuscular Junction via Transforming Growth Factor-1. Journal of Neuroscience, 28(39), 9599-9609. http://doi.org/10.1523/JNEUROSCI.258908.2008

Finzsch, M., Schreiner, S., Kichko, T., Reeh, P., Tamm, E. R., Bösl, M. R., Meijer, D., \& Wegner, M. (2010). Sox10 is required for Schwann cell identity and progression beyond the immature Schwann cell stage. The Journal of Cell Biology, 189(4), 701712. http://doi.org/10.1083/jcb.200912142

Foote, A. K. \& Blakemore, W. F. (2005). Inflammation stimulates remyelination in areas of chronic demyelination. Brain, $128(3), \quad 528-539$. http://doi.org/10.1093/brain/awh417

Foster, R. E., Kocsis, J. D., Malenka, R. C., \& Waxman, S. G. (1980). Lysophosphatidyl choline-induced focal demyelination in the rabbit corpus callosum. Electronmicroscopic observations. Journal of the Neurological Sciences, 48(2), 221-231.

Frohman, E. M., Racke, M. K., \& Raine, C. S. (2006). Multiple sclerosis--the plaque and its pathogenesis. The New England Journal of Medicine, 354(9), 942-955. 
Gajofatto, A., \& Benedetti, M. D. (2015). Treatment strategies for multiple sclerosis: When to start, when to change, when to stop? World Journal of Clinical Cases, 3(7), 545-555. http://doi.org/10.12998/wjcc.v3.i7.545

Gamble, H. J. (1966). Further electron microscope studies of human foetal peripheral nerves. Journal of Anatomy, 100(Pt 3), 487-502.

Gamble, H. J., \& Breathnach, A. S. (1965). An electron-microscope study of human foetal peripheral nerves. Journal of Anatomy, 99(Pt 3), 573-584.

Gamble, H. J., \& Eames, R. A. (1964). An electron microscope study of the connective tissues of human peripheral nerve. Journal of Anatomy, 98, 655-663.

Garbay, B., Heape, A. M., Sargueil, F., \& Cassagne, C. (2000). Myelin synthesis in the peripheral nervous system. Progress in Neurobiology, 61(3), 267-304.

Garratt, A. N., Britsch, S., \& Birchmeier, C. (2000a). Neuregulin, a factor with many functions in the life of a schwann cell. BioEssays, 22(11), 987-996. http://doi.org/10.1002/1521-1878(200011)22:11<987::AID-BIES5>3.0.CO;2-5

Garratt, A. N., Voiculescu, O., Topilko, P., Charnay, P., \& Birchmeier, C. (2000b). A dual role of erbB2 in myelination and in expansion of the schwann cell precursor pool. The Journal of Cell Biology, 148(5), 1035-1046. 
Gibson, E. M., Purger, D., Mount, C. W., Goldstein, A. K., Lin, G. L., Wood, L. S., Inema, I., Miller, S., Bieri, G., Zuchero, B., Barres, B. A., Woo, P. J., Vogel, H., \& Monje, M. (2014). Neuronal Activity Promotes Oligodendrogenesis and Adaptive Myelination in the Mammalian Brain. Science, 344(6183), 1252304-1252304. http://doi.org/10.1126/science. 1252304

Girard, C., Bemelmans, A.-P., Dufour, N., Mallet, J., Bachelin, C., Nait-Oumesmar, B., Baron-Van Evercooren, A., \& Lachapelle, F. (2005). Grafts of Brain-Derived Neurotrophic Factor and Neurotrophin 3-Transduced Primate Schwann Cells Lead to Functional Recovery of the Demyelinated Mouse Spinal Cord. Journal of Neuroscience, 25(35), 7924-7933. http://doi.org/10.1523/JNEUROSCI.489004.2005

Gregg, C., Shikar, V., Larsen, P., Mak, G., Chojnacki, A., Yong, V. W., \& Weiss, S. (2007). White Matter Plasticity and Enhanced Remyelination in the Maternal CNS. Journal of Neuroscience, $\quad 27(8), \quad 1812-1823$. http://doi.org/10.1523/JNEUROSCI.4441-06.2007

Griffin, J. W., Stoll, G., Li, C. Y., Tyor, W., \& Cornblath, D. R. (1990). Macrophage responses in inflammatory demyelinating neuropathies. Annals of Neurology, 27 Suppl, S64-8. 
Halata, Z., Grim, M., \& Christ, B. (1990). Origin of spinal cord meninges, sheaths of peripheral nerves, and cutaneous receptors including Merkel cells. An experimental and ultrastructural study with avian chimeras. Anatomy and Embryology, 182(6), $529-537$.

Hall, S. M. (1972). The effect of injections of lysophosphatidyl choline into white matter of the adult mouse spinal cord. Journal of Cell Science, 10(2), 535-546.

Haninec, P. (1988). Study of the origin of connective tissue sheaths of peripheral nerves in the limb of avian embryos. Anatomy and Embryology, 178(6), 553-557.

Hardy, R. J., Lazzarini, R. A., Colman, D. R., \& Friedrich, V. L. (1996). Cytoplasmic and nuclear localization of myelin basic proteins reveals heterogeneity among oligodendrocytes. Journal of Neuroscience Research, 46(2), 246-257. http://doi.org/10.1002/(SICI)1097-4547(19961015)46:2\&lt;246::AIDJNR13\&gt;3.0.CO;2-0

Harlow, D. E., Honce, J. M., \& Miravalle, A. A. (2015). Remyelination Therapy in Multiple Sclerosis. Frontiers in Neurology, 6(8), 275. http://doi.org/10.1002/ana.22320

Hartman, S., \& Rosenzweig, T. M. (2010). Disease-modifying therapy in adult relapsingremitting multiple sclerosis. Formularly Journal Clinical Pharmacology, 45, 252. 
Hawkins, R. A., O'Kane, R. L., Simpson, I. A., \& Viña, J. R. (2006). Structure of the blood-brain barrier and its role in the transport of amino acids. The Journal of Nutrition, 136(1 Suppl), 218S-26S.

Helker, C. S. M., Schuermann, A., Karpanen, T., Zeuschner, D., Belting, H. G., Affolter, M., Schulte-Merker, S., \& Herzog, W. (2013). The zebrafish common cardinal veins develop by a novel mechanism: lumen ensheathment. Development, 140(13), 27762786. http://doi.org/10.1242/dev.091876

Henderson, A. P. D., Barnett, M. H., Parratt, J. D. E., \& Prineas, J. W. (2009). Multiple sclerosis: Distribution of inflammatory cells in newly forming lesions. Annals of Neurology, 66(6), 739-753. http://doi.org/10.1002/ana.21800

Hendry, I. A., Hill, C. E., \& Watters, D. J. (1986). Long-term retention of Fast Blue in sympathetic neurones after axotomy and regeneration--demonstration of incorrect reconnections. Brain Research, 376(2), 292-298.

Hill, K. E., Zollinger, L. V., Watt, H. E., Carlson, N. G., \& Rose, J. W. (2004). Inducible nitric oxide synthase in chronic active multiple sclerosis plaques: distribution, cellular expression and association with myelin damage. Journal of Neuroimmunology, 151(1-2), 171-179.http://doi.org/10.1016/j.jneuroim.2004.02.005

Hill, R. A., Patel, K. D., Medved, J., Reiss, A. M., \& Nishiyama, A. (2013). NG2 Cells in White Matter But Not Gray Matter Proliferate in Response to PDGF. Journal of 
Neuroscience, 33(36), 14558-14566. http://doi.org/10.1523/JNEUROSCI.200112.2013

Hines, J. H., Ravanelli, A. M., Schwindt, R., \& Scott, E. K. (2015). Neuronal activity biases axon selection for myelination in vivo. Nature. http://doi.org/10.1038/nn.3992

Hinks, G. L., \& Franklin, R. J. M. (2000). Delayed Changes in Growth Factor Gene Expression during Slow Remyelination in the CNS of Aged Rats. Molecular and Cellular Neuroscience, 16(5), 542-556. http://doi.org/10.1006/mcne.2000.0897

Hirano, A., \& Llena, J. (2006). Fine structure of neuronal and glial processes in neuropathology. Neuropathology : Official Journal of the Japanese Society of Neuropathology, 26(1), 1-7. http://doi.org/10.1083/jcb.56.2.478

Hirano, A., Zimmerman, H. M., \& Levine, S. (1965). The fine structure of cerebral fluid accumulation. IX. Edema following silver nitrate implantation. The American Journal of Pathology, 47(4), 537-548.

Ho, D. M., Chan, J., Bayliss, P., \& Whitman, M. (2006). Inhibitor-resistant type I receptors reveal specific requirements for TGF- $\beta$ signaling in vivo. Developmental Biology, 295(2), 730-742. http://doi.org/10.1016/j.ydbio.2006.03.050 
Honjo, Y., Payne, L., \& Eisen, J. S. (2011). Somatosensory mechanisms in zebrafish lacking dorsal root ganglia. Journal of Anatomy, 218(3), 271-276. http://doi.org/10.1111/j.1469-7580.2010.01337.x

Howe, K., Clark, M. D., Torroja, C. F., Torrance, J., Berthelot, C., Muffato, M., Collins, J. E., Humphray, S., McLaren, K., Matthews, L., McLaren, S., Sealy, I., Caccamo, M., Churcher, C., Scott, C., Barrett, J. C., Koch, R., Rauch, G.-J., White, S., Chow, W., Killian, B., Quintais, L. T., Guerra-Assunção, Zhou, Y., Gu, Y., Yen, J., Vogel, J.-H., Eyre, T., Redmond, S., Banerjee, R., Chi, J., Fu, B., Langley, E., Maguire, S. F., Laird, G. K., Lloyd, D., Kenyon, E., Donaldson, S., Sehra, H., Almeida-King, J., Loveland, J., Trevanion, S., Jones, M., Quail, M., Willey, D., Hunt, A., Burton, J., Sims, S., McLay, K., Plumb, B., Davis, J., Clee, C., Oliver, K., Clark, R., Riddle, C., Elliot, D., Threadgold, G., Harden, G., Ware, D., Begum, S., Mortimore, B., Kerry, G., Heath, P., Phillimore, B., Tracey, A., Corby, N., Dunn, M., Johnson, C., Wood, J., Clark, S., Pelan, S., Griffiths, G., Smith, M., Glithero, R., Howden, P., Barker, N., Lloyd, C., Stevens, C., Harley, J., Holt, K., Panagiotidis, G., Lovell, J., Beasley, H., Henderson, C., Gordon, D., Auger, K., Wright, D., Collins, J., Raisen, C., Dyer, L., Leung, K., Robertson, L., Ambridge, K., Leongamornlert, D., McGuire, S., Gilderthorp, R., Griffiths, C., Manthravadi, D., Nichol, S., Barker, G., Whitehead, S., Kay, M., Brown, J., Murnane, C., Gray, E., Humphries, M., Sycamore, N., Barker, D., Saunders, D., Wallis, J., Babbage, A., Hammond, S., Mashreghi-Mohammadi, M., Barr, L., Martin, S., Wray, P., Ellington, A., Matthews, N., Ellwood, M., Woodmansey, R., Clark, G., Cooper., J. D., Tromans, A., Grafham, D., Skuce, C., 
Pandian, R., Andrews, R., Harrison, E., Kimberley, A., Garnett, J., Fosker, N., Hall, R., Garner, P., Kelly., D., Bird., C., Palmer, S., Gehring, I., Berger, A., Dooley, C. M., Ersan-Ürün, Z., Eser, C., Geiger, H., Geisler, M., Karotki, L., Kirn., A., Konantz, J., Konantz, M., Oberlănder, M., Rudolph-Geiger, S., Teucke, M., Lanz, C., Raddatz, G., Osoegawa, K., Zhu, B., Rapp, A., Widaa, S., Langford, C., Yang, F., Schuster, S. C., Carter, N. P., Harrow, J., Ning, Z., Herrero, J., Searle, S. M. J., Enright, A., Geisler, R., Plasterk, R. H. A., Lee, C., Westerfield, M., de Jong, P. J., Zon, L. I., Postlethwait, J. H., Nüsslein-Volhard, C., Hubbard, T. J. P., Crollius, H. R., Rogers, J., \& Stemple, D. L. (2013). The zebrafish reference genome sequence and its relationship to the human genome. Nature, 1-7. http://doi.org/10.1038/nature12111

Höke, A., Redett, R., Hameed, H., Jari, R., Zhouööö, C., Li, Z. B., Griffin, J. W., \& Brusöart, T. M. (2006). Schwann Cells Express Motor and Sensory Phenotypes That Regulate Axon Regeneration. Journal of Neuroscience, 26(38), 9646-9655. http://doi.org/10.1523/JNEUROSCI.1620-06.2006

Hughes, E. G., Kang, S. H., Fukaya, M., \& Bergles, D. E. (2013). Oligodendrocyte progenitors balance growth with self-repulsion to achieve homeostasis in the adult brain. Nature Neuroscience, 16(6), 668-676. http://doi.org/10.1038/nn.3390

Hughes, R. A. C. (2002). Peripheral Neuropathy. British Medical Journal, 324, 466-469. Retrieved from http://www.ncbi.nlm.nih.gov/pmc/articles/PMC1122393/pdf/466.pdf 
Huitinga, I., van Rooijen, N., De Groot, C. J., Uitdehaag, B. M., \& Dijkstra, C. D. (1990). Suppression of experimental allergic encephalomyelitis in Lewis rats after elimination of macrophages. The Journal of Experimental Medicine, 172(4), 10251033.

Irwin, J. A., Erisir, A., \& Kwon, I. (2016). Oral Triphenylmethane Food Dye Analog, Brilliant Blue G, Prevents Neuronal Loss in APPSwDI/NOS2-/- Mouse Model. Current Alzheimer Research, 13(6), 663-677.

Jeffery, N. D., \& Blakemore, W. F. (1995). Remyelination of mouse spinal cord axons demyelinated by local injection of lysolecithin. Journal of Neurocytology, 24(10), $775-781$.

Jenkins, S. J., Ruckerl, D., Cook, P. C., Jones, L. H., Finkelman, F. D., van Rooijen, N., MacDonald, A. S., \& Allen, J. E. (2011). Local Macrophage Proliferation, Rather than Recruitment from the Blood, Is a Signature of TH2 Inflammation. Science, 332(6035), 1284-1288. http://doi.org/10.1126/science.1204351

Jessen, K. R., \& Mirsky, R. (1991). Schwann cell precursors and their development. Glia, 4, 185-194.

Jessen, K. R., \& Mirsky, R. (1997). Embryonic Schwann cell development: the biology of Schwann cell precursors and early Schwann cells. Journal of Anatomy, 191 (Pt 4), $501-505$. 
Jessen, K. R., \& Mirsky, R. (1998). Origin and early development of Schwann cells. Microscopy Research and Technique, 41(5), 393-402.

Jessen, K. R., \& Mirsky, R. (1999). Schwann cells and their precursors emerge as major regulators of nerve development. Trends in Neurosciences, 22(9), 402-410.

Jessen, K. R., \& Mirsky, R. (2005). The origin and development of glial cells in peripheral nerves. Nature Reviews Neuroscience, 6(9), 671-682. http://doi.org/10.1038/nrn1746

Jessen, K. R., \& Mirsky, R. (2010). Control of Schwann cell myelination. F1000 Biology Reports. http://doi.org/10.3410/B2-19

Jesuraj, N. J., Nguyen, P. K., Wood, M. D., Moore, A. M., Borschel, G. H., Mackinnon, S. E., \& Sakiyama-Elbert, S. E. (2011). Differential gene expression in motor and sensory Schwann cells in the rat femoral nerve. Journal of Neuroscience Research, 90(1), 96-104. http://doi.org/10.1002/jnr.22752

Joseph, N. M., Mukouyama, Y.-S., Mosher, J. T., Jaegle, M., Crone, S. A., Dormand, E.L., Lee, K.-F., Meijer, D., Anderson, D. J., Morrison, S. J. (2004). Neural crest stem cells undergo multilineage differentiation in developing peripheral nerves to generate endoneurial fibroblasts in addition to Schwann cells. Development, 131(22), 55995612. http://doi.org/10.1242/dev.01429 
Jung, J., Cai, W., Lee, H. K., Pellegatta, M., Shin, Y. K., Jang, S. Y., Suh, D. J., Wrabetz, L., Feltri, M. L., \& Park, H. T. (2011). Actin Polymerization Is Essential for Myelin Sheath Fragmentation during Wallerian Degeneration. Journal of Neuroscience, 31(6), 2009-2015. http://doi.org/10.1523/JNEUROSCI.4537-10.2011

Kaufmann, W., Bolon, B., Bradley, A., Butt, M., Czasch, S., Garman, R. H., George, C., Gröters, S., Krinke, G., Little, P., McKay, J., Narama, I., Rao, D., Shibutani, M., \& Sills, R. (2012). Proliferative and Nonproliferative Lesions of the Rat and Mouse Central and Peripheral Nervous Systems. Toxicologic Pathology, 40, 87S-157S. http://doi.org/10.1016/S0021-9975(08)80308-8

Kearns, C. A., Ravanelli, A. M., Cooper, K., \& Appel, B. (2015). Fbxw7 Limits Myelination by Inhibiting mTOR Signaling. Journal of Neuroscience, 35(44), 14861-14871. http://doi.org/10.1523/JNEUROSCI.4968-14.2015

Keirstead, H. S., \& Blakemore, W. F. (1997). Identification of post-mitotic oligodendrocytes incapable of remyelination within the demyelinated adult spinal cord. Journal of Neuropathology and Experimental Neurology, 56(11), 1191-1201.

Keough, M. B., Jensen, S. K., \& Yong, V. W. (2015). Experimental Demyelination and Remyelination of Murine Spinal Cord by Focal Injection of Lysolecithin. Journal of Visualized Experiments, (97). http://doi.org/10.3791/52679 
Kimmel, C. B., Ballard, W. W., Kimmel, S. R., Ullmann, B., \& Schilling, T. F. (1995). Stages of embryonic development of the zebrafish. Developmental Dynamics, 203(3), 253-310. http://doi.org/10.1002/aja.1002030302

Kirby, B. B., Takada, N., Latimer, A. J., Shin, J., Carney, T. J., Kelsh, R. N., \& Appel, B. (2006). In vivo time-lapse imaging shows dynamic oligodendrocyte progenitor behavior during zebrafish development. Nature Neuroscience, 9(12), 1506-1511. http://doi.org/10.1038/nn1803

Kitada, M., \& Rowitch, D. H. (2006). Transcription factor co-expression patterns indicate heterogeneity of oligodendroglial subpopulations in adult spinal cord. Glia, 54(1), 35-46. http://doi.org/10.1002/glia.20354

Kitisin, K., Saha, T., Blake, T., Golestaneh, N., Deng, M., Kim, C., Tang, Y., Shetty, K., Mishra, B., \& Mishra, L. (2007). Tgf-Beta signaling in development. Science Signaling 2007 (399), cm1. http://doi.org/10.1126/stke.3992007cm1

Kotter, M. R., Li, W.-W., Zhao, C., \& Franklin, R. J. M. (2006). Myelin impairs CNS remyelination by inhibiting oligodendrocyte precursor cell differentiation. Journal of Neuroscience, 26(1), 328-332. http://doi.org/10.1523/JNEUROSCI.2615-05.2006

Kotter, M. R., Setzu, A., Sim, F. J., Van Rooijen, N., \& Franklin, R. J. M. (2001). Macrophage depletion impairs oligodendrocyte remyelination following lysolecithininduced demyelination. Glia, 35(3), 204-212. http://doi.org/10.1002/glia.1085 
Kotter, M. R., Zhao, C., Van Rooijen, N., \& Franklin, R. J. M. (2005). Macrophagedepletion induced impairment of experimental CNS remyelination is associated with a reduced oligodendrocyte progenitor cell response and altered growth factor expression. Neurobiology of Disease, 18(1), 166-175.

Kremer, D., Küry, P., \& Dutta, R. (2015). Promoting remyelination in multiple sclerosis: current drugs and future prospects. Multiple Sclerosis Journal, 21(5), 541-549. http://doi.org/10.1177/1352458514566419

Kristensson, K., \& Olsson, Y. (1971). The perineurium as a diffusion barrier to protein tracers. Acta Neuropathologica, 17(2), 127-138.

Kucenas, S., Snell, H., \& Appel, B. (2008a). nkx2.2a promotes specification and differentiation of a myelinating subset of oligodendrocyte lineage cells in zebrafish. Neuron Glia Biology, 4(2), 71-81. http://doi.org/10.1017/S1740925X09990123

Kucenas, S., Takada, N., Park, H.-C., Woodruff, E., Broadie, K., \& Appel, B. (2008b). CNS-derived glia ensheath peripheral nerves and mediate motor root development. Nature Neuroscience, 11(2), 143-151. http://doi.org/10.1038/nn2025

Kucenas, S., Wang, W. D., Knapik, E. W., \& Appel, B. (2009). A Selective Glial Barrier at Motor Axon Exit Points Prevents Oligodendrocyte Migration from the Spinal Cord. Journal of Neuroscience, 29(48), 15187-15194. 
Kucharova, K., Chang, Y., Boor, A., Yong, V. W., \& Stallcup, W. B. (2011). Reduced inflammation accompanies diminishedmyelin damage and repair in the NG2 null mousespinal cord. Journal of Neuroinflammation, $8(1), \quad 158$. http://doi.org/10.1186/1742-2094-8-158

Kuhlbrodt, K., Herbarth, B., Sock, E., Hermans-Borgmeyer, I., \& Wegner, M. (1998). Sox10, a novel transcriptional modulator in glial cells. Journal of Neuroscience, $18(1), 237-250$.

Kuhlmann, T., Miron, V., Cuo, Q., Wegner, C., Antel, J., \& Bruck, W. (2008). Differentiation block of oligodendroglial progenitor cells as a cause for remyelination failure in chronic multiple sclerosis. Brain, 131(7), 1749-1758. http://doi.org/10.1093/brain/awn096

Kuspert, M., Weider, M., Muller, J., Hermans-Borgmeyer, I., Meijer, D., \& Wegner, M. (2012). Desert Hedgehog Links Transcription Factor Sox10 to Perineurial Development. Journal of Neuroscience, 32(16), 5472-5480. http://doi.org/10.1523/JNEUROSCI.5759-11.2012

Lachapelle, F., Bachelin, C., Moissonnier, P., Nait-Oumesmar, B., Hidalgo, A., Fontaine, D., \& Baron-Van Evercooren, A. (2005). Failure of remyelination in the nonhuman primate optic nerve. Brain Pathology (Zurich, Switzerland), 15(3), 198-207. 
Lampron, A., Larochelle, A., Laflamme, N., Préfontaine, P., Plante, M.-M., Sánchez, M. G., Yong, V. W., Stys, P. K., Tremblay, M.-E., \& Rivest, S. (2015). Inefficient clearance of myelin debris by microglia impairs remyelinating processes. Journal of Experimental Medicine, 212(4), 481-495. http://doi.org/10.1084/jem.20132477

Larson, T. A., Gordon, T. N., Lau, H. E., \& Parichy, D. M. (2010). Defective adult oligodendrocyte and Schwann cell development, pigment pattern, and craniofacial morphology in puma mutant zebrafish having an alpha tubulin mutation. Developmental Biology, 346(2), 296-309. http://doi.org/10.1016/j.ydbio.2010.07.035

Lassmann, H., Bruck, W., \& Lucchinetti, C. (2001). Heterogeneity of multiple sclerosis pathogenesis: implications for diagnosis and therapy. Trends in Molecular Medicine, 7(3), 115-121.

Lau, L. W., Keough, M. B., Haylock-Jacobs, S., Cua, R., Döring, A., Sloka, S., Stirling, D. P., Rivest, S., \& Yong, V. W. (2012). Chondroitin sulfate proteoglycans in demyelinated lesions impair remyelination. Annals of Neurology, 72(3), 419-432. http://doi.org/10.1002/ana.23599

Le Douarin, N. M., \& Dupin, E. (2003). Multipotentiality of the neural crest. Current Opinion in Genetics \& Development, 13(5), 529-536. http://doi.org/10.1016/j.gde.2003.08.002 
Le Douarin, N., Dulac, C., Dupin, E., \& Cameron-Curry, P. (1991). Glial cell lineages in the neural crest. Glia, 4(2), 175-184. http://doi.org/10.1002/glia.440040209

Lee, K. F., Simon, H., Chen, H., Bates, B., Hung, M. C., \& Hauser, C. (1995). Requirement for neuregulin receptor erbB2 in neural and cardiac development. Nature, 378(6555), 394-398. http://doi.org/10.1038/378394a0

Lewis, G. M., \& Kucenas, S. (2013). Motor Nerve Transection and Time-lapse Imaging of Glial Cell Behaviors in Live Zebrafish. Journal of Visualized Experiments, (76). http://doi.org/10.3791/50621

Lewis, G. M., \& Kucenas, S. (2014). Perineurial glia are essential for motor axon regrowth following nerve injury. Journal of Neuroscience, 34(38), 12762-12777. http://doi.org/10.1523/JNEUROSCI.1906-14.2014

Liddelow, S. A., Dziegielewska, K. M., Ek, C. J., Johansson, P. A., Potter, A. M., \& Saunders, N. R. (2009). Cellular transfer of macromolecules across the developing choroid plexus of Monodelphis domestica. European Journal of Neuroscience, 29(2), 253-266. http://doi.org/10.1111/j.1460-9568.2008.06571.x

Lin, W., Burgess, R. W., Dominguez, B., Pfaff, S. L., Sanes, J. R., \& Lee, K. F. (2001). Distinct roles of nerve and muscle in postsynaptic differentiation of the neuromuscular synapse. Nature, 410(6832), 1057-1064. 
Liu, Z., Hu, X., Cai, J., Liu, B., Peng, X., Wegner, M., \& Qiu, M. (2007). Induction of oligodendrocyte differentiation by Olig2 and Sox10: Evidence for reciprocal interactions and dosage-dependent mechanisms. Developmental Biology, 302(2), 683-693. http://doi.org/10.1016/j.ydbio.2006.10.007

Locatelli, G., Baggiolini, A., Schreiner, B., Palle, P., Waisman, A., Becher, B., \& Buch, T. (2015). Mature oligodendrocytes actively increase in vivo cytoskeletal plasticity following CNS damage. Journal of Neuroinflammation, 12, 62. http://doi.org/10.1186/s12974-015-0271-2

Lucchinetti, C., Bruck, W., Parisi, J., Scheithauer, B., Rodriguez, M., \& Lassmann, H. (2000). Heterogeneity of multiple sclerosis lesions: implications for the pathogenesis of demyelination. Annals of Neurology, 47(6), 707-717.

Lyons, D. A., Naylor, S. G., Mercurio, S., Dominguez, C., \& Talbot, W. S. (2008). KBP is essential for axonal structure, outgrowth and maintenance in zebrafish, providing insight into the cellular basis of Goldberg-Shprintzen syndrome. Development, 135(3), 599-608. http://doi.org/10.1242/dev.012377

Lyons, D. A., Naylor, S. G., Scholze, A., \& Talbot, W. S. (2009). Kiflb is essential for mRNA localization in oligodendrocytes and development of myelinated axons. Nature Publishing Group, 41(7), 854-858. http://doi.org/10.1038/ng.376 
Lyons, D. A., Pogoda, H.-M., Voas, M. G., Woods, I. G., Diamond, B., Nix, R., Arana, N., Jacobs, J., \& Talbot, W. S. (2005). erbb3 and erbb2 Are Essential for Schwann Cell Migration and Myelination in Zebrafish. Current Biology, 15(6), 513-524. http://doi.org/10.1016/j.cub.2005.02.030

Marques, S., Zeisel, A., Codeluppi, S., van Bruggen, D., Mendanha Falcão, A., Xiao, L., Li, H., Häring, M., Hochgerner, H., Romanov, R. A., Gyllborg, D., MuñozManchado, A. B., La Manno, G., Lönnerberg, P., Floriddia, E. M., Rezayee, F., Ernfors, P., Arenas, E., Hjerling-Leffler, J., Harkany, T., Richardson, W. D., Linnarsson, S., \& Castelo-Branco, G. (2016). Oligodendrocyte heterogeneity in the mouse juvenile and adult central nervous system. Science, 352(6291), 1326-1329. http://doi.org/10.1126/science.aaf6463

Mason, J. L., Suzuki, K., Chaplin, D. D., \& Matsushima, G. K. (2001). Interleukin-1beta promotes repair of the CNS. Journal of Neuroscience, 21(18), 7046-7052.

Matsumoto, Y., Ohmori, K., \& Fujiwara, M. (1992). Microglial and astroglial reactions to inflammatory lesions of experimental autoimmune encephalomyelitis in the rat central nervous system. Journal of Neuroimmunology, 37(1-2), 23-33.

Matsushima, G. K., \& Morell, P. (2001). The neurotoxicant, cuprizone, as a model to study demyelination and remyelination in the central nervous system. Brain Pathology. 
Maurel, P., \& Salzer, J. L. (2000). Axonal regulation of Schwann cell proliferation and survival and the initial events of myelination requires PI 3-kinase activity. Journal of Neuroscience, 20(12), 4635-4645.

Mayoral, S. R., \& Chan, J. R. (2016). ScienceDirectThe environment rules: spatiotemporal regulation of oligodendrocyte differentiation. Current Opinion in Neurobiology, 39, 47-52. http://doi.org/10.1016/j.conb.2016.04.002

McFarland, H. F., \& Martin, R. (2007). Multiple sclerosis: a complicated picture of autoimmunity. Nature Immunology, 8(9), 913-919. http://doi.org/10.1038/ni1507

McGraw, H. F., Snelson, C. D., Prendergast, A., Suli, A., \& Raible, D. W. (2012). Postembryonic neuronal addition in zebrafish dorsal root ganglia is regulated by Notch signaling. Neural Development, 7, 23. http://doi.org/10.1186/1749-8104-7-23

McKay, J. S., Blakemore, W. F., \& Franklin, R. J. (1998). Trapidil-mediated inhibition of CNS remyelination results from reduced numbers and impaired differentiation of oligodendrocytes. Neuropathology and Applied Neurobiology, 24(6), 498-506.

McMartin, D. N., O'Donoghue, J. L., Morrissey, R., \& Fix, A. S. (1997). Nonproliferative lesions of the nervous system in rats. Guides for Toxicologic Pathology. 
McTigue, D. M., \& Tripathi, R. B. (2008). The life, death, and replacement of oligodendrocytes in the adult CNS. Journal of Neurochemistry, 107(1), 1-19.

Meeker, N., Hutchinson, S., Ho, L., \& Trede, N. (2007). Method for isolation of PCRready genomic DNA from zebrafish tissues. BioTechniques, 43(5), 610-614. http://doi.org/10.2144/000112619

Mei, F., Fancy, S. P. J., Shen, Y.-A. A., Niu, J., Zhao, C., Presley, B., Miao, E., Lee, S., Mayoral, S. R., Redmond, S. A., Etxeberria, A., Xiao, L., Franklin, R. J. M., Green, A., Hauser, S. L., \& Chan, J. R. (2014). Micropillar arrays as a high-throughput screening platform for therapeutics in multiple sclerosis. Nature Medicine, 20(8), 954-960. http://doi.org/10.1038/nm.3618

Mensch, S., Baraban, M., Almeida, R., Czopka, T., Ausborn, J., Manira, El, A., \& Lyons, D. A. (2015). Synaptic vesicle release regulates myelin sheath number of individual oligodendrocytes in vivo. Nature Neuroscience, 18(5), 628-630. http://doi.org/10.1038/nn.3991

Michailov, G. V. (2004). Axonal Neuregulin-1 Regulates Myelin Sheath Thickness. Science, 304(5671), 700-703. http://doi.org/10.1126/science.1095862

Miller, R. H., \& Fyffe-Maricich, S. L. (2010). Restoring the balance between disease and repair in multiple sclerosis: insights from mouse models. Disease Models and Mechanisms, 3(9-10), 535-539. http://doi.org/10.1242/dmm.001958 
Miron, V. E., Boyd, A., Zhao, J.-W., Yuen, T. J., Ruckh, J. M., Shadrach, J. L., van Wijngaarden, P., Wagers, A. J., Williams, A., Franklin, R. J. M., \& ffrench-Constant, C. (2013). M2 microglia and macrophages drive oligodendrocyte differentiation during CNS remyelination. Nature Neuroscience, 16(9), 1211-1218.

Mirsky, R., Jessen, K. R., Brennan, A., Parkinson, D., Dong, Z., Meier, C., Parmantier, E., \& Lawson, D. (2002). Schwann cells as regulators of nerve development. Journal of Physiology, Paris, 96(1-2), 17-24.

Mirsky, R., Parmantier, E., McMahon, A. P., \& Jessen, K. R. (1999). Schwann cellderived desert hedgehog signals nerve sheath formation. Annals of the New York Academy of Sciences, 883, 196-202.

Monk, K. R., Naylor, S. G., Glenn, T. D., Mercurio, S., Perlin, J. R., Dominguez, C., Moens, C. B., \& Talbot, W. S. (2009). A G Protein-Coupled Receptor Is Essential for Schwann Cells to Initiate Myelination. Science, 325(5946), 1402-1405. http://doi.org/10.1126/science.1173474

Monk, K. R., Oshima, K., Jors, S., Heller, S., \& Talbot, W. S. (2011). Gpr126 is essential for peripheral nerve development and myelination in mammals. Development, 138(13), 2673-2680. http://doi.org/10.1242/dev.062224 
Montero-Balaguer, M., Lang, M. R., Sachdev, S. W., Knappmeyer, C., Stewart, R. A., De La Guardia, A., Hatzopoulos, A. K., \& Knapik, E. W. (2006). The mother superiormutation ablates foxd3activity in neural crest progenitor cells and depletes neural crest derivatives in zebrafish. Developmental Dynamics, 235(12), 3199-3212. http://doi.org/10.1002/dvdy.20959

Morris, J. K. J., Lin, W. W., Hauser, C. C., Marchuk, Y. Y., Getman, D. D., \& Lee, K. F. K. (1999). Rescue of the cardiac defect in ErbB2 mutant mice reveals essential roles of ErbB2 in peripheral nervous system development. Neuron, 23(2), 273-283.

Mujtaba, T., Mayer-Proschel, M., \& Rao, M. S. (1998). A common neural progenitor for the CNS and PNS. Developmental Biology, 200(1), 1-15. http://doi.org/10.1006/dbio.1998.8913

Münzel, E. J., Becker, C. G., Becker, T., \& Williams, A. (2014). Zebrafish regenerate full thickness optic nerve myelin after demyelination, but this fails with increasing age. Acta Neuropathologica Communications, 2, 77. http://doi.org/10.1186/s40478-0140077-y

Myers, P. Z., Eisen, J. S., \& Westerfield, M. (1986). Development and axonal outgrowth of identified motoneurons in the zebrafish. Journal of Neuroscience, 6(8), 22782289. 
Nait-Oumesmar, B., Decker, L., Lachapelle, F., Avellana-Adalid, V., Bachelin, C., \& Baron-Van Evercooren, A. (1999). Progenitor cells of the adult mouse subventricular zone proliferate, migrate and differentiate into oligodendrocytes after demyelination. The European Journal of Neuroscience, 11(12), 4357-4366.

Nathan, C. F. (1987). Secretory products of macrophages. Journal of Clinical Investigation, 79(2), 319-326. http://doi.org/10.1172/JCI112815

Nave, K. A. (2010). Myelination and support of axonal integrity by glia. Nature, 468(7321), 244-252. http://doi.org/10.1038/nature09614

Nave, K. A., \& Salzer, J. L. (2006). Axonal regulation of myelination by neuregulin 1. Current Opinion in Neurobiology, 16(5), 492-500. http://doi.org/10.1016/j.conb.2006.08.008

Nawaz, S., Sánchez, P., Schmitt, S., Snaidero, N., Mitkovski, M., Velte, C., Brückner, B. R., Alexopoulos, I., Czopka, T., Jung, S. Y., Rhee, J. S., Janshoff, A., Witke, W., Schaap, I. A. T., Lyons, D. A., \& Simons, M. (2015). Actin Filament Turnover Drives Leading Edge Growth during Myelin Sheath Formation in the Central Nervous System. Developmental Cell, 34(2), 139-151. http://doi.org/10.1016/j.devcel.2015.05.013 
Neumann, H., Kotter, M. R., \& Franklin, R. J. M. (2008). Debris clearance by microglia: an essential link between degeneration and regeneration. Brain, 132(2), 288-295. http://doi.org/10.1093/brain/awn109

Newbern, J., \& Birchmeier, C. (2010). Nrg1/ErbB signaling networks in Schwann cell development and myelination. Seminars in Cell \& Developmental Biology, 21(9), 922-928. http://doi.org/10.1016/j.semcdb.2010.08.008

Nguyen, Q. T., Sanes, J. R., \& Lichtman, J. W. (2002). Pre-existing pathways promote precise projection patterns. Nature Neuroscience, 5(9), 861-867. http://doi.org/10.1038/nn905

Noseworthy, J. H., Lucchinetti, C., Rodriguez, M., \& Weinshenker, B. G. (2000). Multiple sclerosis. The New England Journal of Medicine, 343(13), 938-952. http://doi.org/10.1056/NEJM200009283431307

Ornelas, I. M., McLane, L. E., Saliu, A., Evangelou, A. V., Khandker, L., \& Wood, T. L. (2016). Heterogeneity in oligodendroglia: Is it relevant to mouse models and human disease? Journal of Neuroscience Research, 94(12), 1421-1433. http://doi.org/10.1007/s10803-016-2803-8

Ousman, S. S., \& David, S. (2000). Lysophosphatidylcholine induces rapid recruitment and activation of macrophages in the adult mouse spinal cord. Glia, 30(1), 92-104. 
Palha, N., Guivel-Benhassine, F., Briolat, V., Lutfalla, G., Sourisseau, M., Ellett, F., Wang, C.-H., Lieschke, G. J., Herbomel, P., Schwartz, O., \& Levraud, J.-P. (2013). Real-Time Whole-Body Visualization of Chikungunya Virus Infection and Host Interferon Response in Zebrafish. PLoS Pathogens, 9(9), e1003619. http://doi.org/10.1371/journal.ppat.1003619.s004

Paratore, C., Goerich, D. E., Suter, U., Wegner, M., \& Sommer, L. (2001). Survival and glial fate acquisition of neural crest cells are regulated by an interplay between the transcription factor Sox10 and extrinsic combinatorial signaling. Development, 128(20), 3949-3961.

Parmantier, E., Lynn, B., Lawson, D., Turmaine, M., Namini, S. S., Chakrabarti, L., McMahon, A. P., Jessen, K. R., \& Mirsky, R. (1999). Schwann cell-derived Desert hedgehog controls the development of peripheral nerve sheaths. Neuron, 23(4), 713724.

Peferoen, L., Kipp, M., van der Valk, P., van Noort, J. M., \& Amor, S. (2014). Oligodendrocyte-microglia cross-talk in the central nervous system. Immunology, 141(3), 302-313. http://doi.org/10.1111/imm.12163

Pereira, J. A., Lebrun-Julien, F., \& Suter, U. (2012). Molecular mechanisms regulating myelination in the peripheral nervous system. Trends in Neurosciences, 35(2), 123134. http://doi.org/10.1016/j.tins.2011.11.006 
Peri, F., \& Nüsslein-Volhard, C. (2008). Live Imaging of Neuronal Degradation by Microglia Reveals a Role for v0-ATPase a1 in Phagosomal Fusion In Vivo. Cell, 133(5), 916-927. http://doi.org/10.1016/j.cell.2008.04.037

Perlin, J. R., Lush, M. E., Stephens, W. Z., Piotrowski, T., \& Talbot, W. S. (2011). Neuronal Neuregulin 1 type III directs Schwann cell migration. Development, 138(21), 4639-4648. http://doi.org/10.1242/dev.068072

Peters, A. (1960). The structure of myelin sheaths in the central nervous system of Xenopus laevis (Daudin). The Journal of Biophysical and Biochemical Cytology, $7(1), 121-126$.

Pick, J., Gerdin, C., \& Delemos, C. (1963). On the ultrastructure of spinal nerve roots in the frog (Rana pipiens). The Anatomical Record, 146(1), 61-84.

Pisharath, H., \& Parsons, M. J. (2009). Nitroreductase-mediated cell ablation in transgenic zebrafish embryos. Methods in Molecular Biology (Clifton, N.J.), 546, 133-143. http://doi.org/10.1007/978-1-60327-977-2_9

Pisharath, H., Rhee, J. M., Swanson, M. A., Leach, S. D., \& Parsons, M. J. (2007). Targeted ablation of beta cells in the embryonic zebrafish pancreas using E. coli nitroreductase. Mechanisms of Development, 124(3), 218-229. http://doi.org/10.1016/j.mod.2006.11.005 
Plessis, Du, D. G., Mouton, Y. M., Muller, C. J., \& Geiger, D. H. (1996). An ultrastructural study of the development of the chicken perineurial sheath. Journal of Anatomy, 189 ( Pt 3), 631-641.

Pozzilli, C., Ruggieri, \& Gasperini, C. (2010). Emerging oral treatments in multiple sclerosis \&ndash; clinical utility of cladribine tablets. Therapeutics and Clinical Risk Management, 391. http://doi.org/10.2147/TCRM.S6639

Prendergast, A., Linbo, T. H., Swarts, T., Ungos, J. M., McGraw, H. F., Krispin, S., Weinstein, B. M., Raible, D. W. (2012). The metalloproteinase inhibitor Reck is essential for zebrafish DRG development. Development, 139(6), 1141-1152. http://doi.org/10.1242/dev.072439

Prineas, J. W., \& Connell, F. (1979). Remyelination in multiple sclerosis. Annals of Neurology, 5(1), 22-31. http://doi.org/10.1002/ana.410050105

Pummi, K. P., Heape, A. M., Grénman, R. A., Peltonen, J. T. K., \& Peltonen, S. A. (2004). Tight Junction Proteins ZO-1, Occludin, and Claudins in Developing and Adult Human Perineurium. Journal of Histochemistry and Cytochemistry, 52(8), 1037-1046. http://doi.org/10.1369/jhc.3A6217.2004

Qi, Y., Cai, J., Wu, Y., Wu, R., Lee, J., Fu, H., Rao, M., Sussel, L., Rubenstein, J., \& Qiu, M. (2001). Control of oligodendrocyte differentiation by the Nkx2.2 homeodomain transcription factor. Development, 128(14), 2723-2733. 
Quarles, R. H., Macklin, W. B., \& Morell, P. (2006). Myelin formation, structure and biochemistry. Basic Neurochemistry: Molecular, Cellular \& Medical Aspects, 51-71.

Quinn, M. T., Parthasarathy, S., \& Steinberg, D. (1988). Lysophosphatidylcholine: a chemotactic factor for human monocytes and its potential role in atherogenesis. Proceedings of the National Academy of Sciences of the United States of America, 85(8), 2805-2809.

Raible, D. W., Wood, A., Hodsdon, W., Henion, P. D., Weston, J. A., \& Eisen, J. S. (1992). Segregation and early dispersal of neural crest cells in the embryonic $\begin{array}{llll}\text { zebrafish. } & \text { Developmental } & \text { Dynamics, } & \text { 195(1), }\end{array}$ http://doi.org/10.1002/aja.1001950104

Raivich, G., \& Banati, R. (2004). Brain microglia and blood-derived macrophages: molecular profiles and functional roles in multiple sclerosis and animal models of autoimmune demyelinating disease. Brain Research Reviews, 46(3), 261-281. http://doi.org/10.1016/j.brainresrev.2004.06.006

Ransohoff, R. M. (2012). Animal models of multiple sclerosis: the good, the bad and the bottom line. Nature Neuroscience, 15(8), 1074-1077. http://doi.org/10.1038/nn.3168

Raphael, A. R., Lyons, D. A., \& Talbot, W. S. (2011). ErbB signaling has a role in radial sorting independent of Schwann cell number. Glia, 59(7), 1047-1055. http://doi.org/10.1002/glia.21175 
Rasband, M. N. (2016). Glial Contributions to Neural Function and Disease. Molecular \& Cellular Proteomics, 15(2), 355-361. http://doi.org/10.1074/mcp.R115.053744

Rawji, K. S., \& Yong, V. W. (2013). The Benefits and Detriments of Macrophages/Microglia in Models of Multiple Sclerosis. Clinical and Developmental Immunology, 2013(5), 1-13. http://doi.org/10.1111/j.1365-2990.2007.00805.x

Remington, L. T., Babcock, A. A., Zehntner, S. P., \& Owens, T. (2007). Microglial Recruitment, Activation, and Proliferation in Response to Primary Demyelination. The American Journal of Pathology, 170(5), 1713-1724. http://doi.org/10.2353/ajpath.2007.060783

Riethmacher, D., Sonnenberg-Riethmacher, E., Brinkmann, V., Yamaai, T., Lewin, G. R., \& Birchmeier, C. (1997a). Severe neuropathies in mice with targeted mutations in the ErbB3 receptor. Nature, 389(6652), 725-730. http://doi.org/10.1038/39593

Riethmacher, D., Sonnenberg-Riethmacher, E., Brinkmann, V., Yamaai, T., Lewin, G. R., \& Birchmeier, C. (1997b). Severe neuropathies in mice with targeted mutations in the ErbB3 receptor. Nature, 389(6652), 725-730. http://doi.org/10.1038/39593

Rinner, W. A., Bauer, J., Schmidts, M., Lassmann, H., \& Hickey, W. F. (1995). Resident microglia and hematogenous macrophages as phagocytes in adoptively transferred experimental autoimmune encephalomyelitis: an investigation using rat radiation bone marrow chimeras. Glia, 14(4), 257-266. http://doi.org/10.1002/glia.440140403 
Rose, J. W., Hill, K. E., Watt, H. E., \& Carlson, N. G. (2004). Inflammatory cell expression of cyclooxygenase-2 in the multiple sclerosis lesion. Journal of Neuroimmunology, 149(1-2), 40-49. http://doi.org/10.1016/j.jneuroim.2003.12.021

Rosenberg, A. F., Isaacman-Beck, J., Franzini-Armstrong, C., \& Granato, M. (2014). Schwann Cells and Deleted in Colorectal Carcinoma Direct Regenerating Motor Axons Towards Their Original Path. Journal of Neuroscience, 34(44), 14668-14681. http://doi.org/10.1523/JNEUROSCI.2007-14.2014

Rosenberg, A. F., Wolman, M. A., Franzini-Armstrong, C., \& Granato, M. (2012). In Vivo Nerve-Macrophage Interactions Following Peripheral Nerve Injury. Journal of Neuroscience, 32(11), 3898-3909. http://doi.org/10.1523/JNEUROSCI.522511.2012

Rosenberg, S. S., Kelland, E. E., Tokar, E., la Torre, De, A. R., \& Chan, J. R. (2008). The geometric and spatial constraints of the microenvironment induce oligodendrocyte differentiation. Proceedings of the National Academy of Sciences of the United States of America, 105(38), 14662-14667. http://doi.org/10.1073/pnas.0805640105

Rowitch, D. H. (2004). Glial specification in the vertebrate neural tube. Nature Reviews Neuroscience, 5(5), 409-419. http://doi.org/10.1038/nrn1389 
Rowitch, D. H., \& Kriegstein, A. R. (2010). Developmental genetics of vertebrate glialcell specification. Nature, 468(7321), 214-222. http://doi.org/10.1038/nature09611

Ruckh, J. M., Zhao, J.-W., Shadrach, J. L., van Wijngaarden, P., Rao, T. N., Wagers, A. J., \& Franklin, R. J. M. (2012). Short Article. Stem Cell, 10(1), 96-103.

Sahel, A. L., Ortiz, F. C., Kerninon, C., Maldonado, P. P., Angulo, M. A. C., \& NaitOumesmar, B. (2015). Alteration of synaptic connectivity of oligodendrocyte precursor cells following demyelination. Frontiers in Cellular Neuroscience, 9(255), 9133. http://doi.org/10.1038/nn1854

Salzer, J. L., Brophy, P. J., \& Peles, E. (2008). Molecular domains of myelinated axons in the peripheral nervous system. Glia, 56(14), 1532-1540. http://doi.org/10.1002/glia.20750

Samanta, J., \& Salzer, J. L. (2015). Myelination: Actin Disassembly Leads the Way. Developmental Cell, 34(2), 129-130. http://doi.org/10.1016/j.devcel.2015.07.006

Saunders, N. R., Dziegielewska, K. M., Møllgård, K., \& Habgood, M. D. (2015). Markers for blood-brain barrier integrity: how appropriate is Evans blue in the twenty-first century and what are the alternatives? Frontiers in Neuroscience, 9(273), 13. http://doi.org/10.1371/journal.pone.0068595 
Saunders, N. R., Ek, C. J., Habgood, M. D., \& Dziegielewska, K. M. (2008). Barriers in the brain: a renaissance? Trends in Neurosciences, 31(6), 279-286. http://doi.org/10.1016/j.tins.2008.03.003

Schafer, D. P., Lehrman, E. K., Kautzman, A. G., Koyama, R., Mardinly, A. R., Yamasaki, R., Ransohoff, R. M., Greenberg, M. E., Barres, B. A., \& Stevens, B. (2012). Microglia Sculpt Postnatal Neural Circuitsin an Activity and ComplementDependent Manner. Neuron, $\quad$ 74(4), 691-705. http://doi.org/10.1016/j.neuron.2012.03.026

Scherer, S. S., Kamholz, J., \& Jakowlew, S. B. (1993). Axons modulate the expression of transforming growth factor-betas in Schwann cells. Glia, 8(4), 265-276. http://doi.org/10.1002/glia.440080407

Schmidt, C., Ohlemeyer, C., Labrakakis, C., Walter, T., Kettenmann, H., \& Schnitzer, J. (1997). Analysis of motile oligodendrocyte precursor cells in vitro and in brain slices. Glia, 20(4), 284-298.

Schmued, L., Bowyer, J., Cozart, M., Heard, D., Binienda, Z., \& Paule, M. (2008). Introducing Black-Gold II, a highly soluble gold phosphate complex with several unique advantages for the histochemical localization of myelin. Brain Research, 1229, 210-217. http://doi.org/10.1016/j.brainres.2008.06.129 
Schönrock, L. M., Kuhlmann, T., Adler, S., Bitsch, A., \& Bruck, W. (1998). Identification of glial cell proliferation in early multiple sclerosis lesions. Neuropathology and Applied Neurobiology, 24(4), 320-330.

Seo, J. H., Maki, T., Maeda, M., Miyamoto, N., Liang, A. C., Hayakawa, K., Pham, L.-D. D., Suwa, F., Taguchi, A., Matsuyama, T., Ihara, M., Kim, K.-W., Lo, E. H., \& Arai, K. (2014). Oligodendrocyte precursor cells support blood-brain barrier integrity via TGF- $\beta$ signaling. PLoS ONE, 9(7), e103174.

Sepp, K. J., Schulte, J., \& Auld, V. J. (2000). Developmental dynamics of peripheral glia in Drosophila melanogaster. Glia, 30(2), 122-133.

Sepp, K. J., Schulte, J., \& Auld, V. J. (2001). Peripheral Glia Direct Axon Guidance across the CNS/PNS Transition Zone. Developmental Biology, 238(1), 47-63. http://doi.org/10.1006/dbio.2001.0411

Shanthaveerappa, T. R., \& Bourne, G. H. (1966). Perineural epithelium: a new concept of its role in the integrity of the peripheral nervous system. Science, 154(3755), 14641467.

Sharghi-Namini, S., Turmaine, M., Meier, C., Sahni, V., Umehara, F., Jessen, K. R., \& Mirsky, R. (2006). The Structural and Functional Integrity of Peripheral Nerves Depends on the Glial-Derived Signal Desert Hedgehog. Journal of Neuroscience, 26(23), 6364-6376. http://doi.org/10.1523/JNEUROSCI.0157-06.2006 
Sharma, K., Selzer, M. E., \& Li, S. (2012). Experimental Neurology. Experimental Neurology, 237(2), 370-378. http://doi.org/10.1016/j.expneurol.2012.07.009

Siegel, G. J., Agranoff, B. W., \& Albers, R. W. (1999). The Myelin Sheath. Basic Neurochemistry (6 ed.). Philadelphia: Lippincott-Raven.

Smith, C. J., Morris, A. D., Welsh, T. G., \& Kucenas, S. (2014). Contact-mediated inhibition between oligodendrocyte progenitor cells and motor exit point glia establishes the spinal cord transition zone. PLoS Biol. http://doi.org/10.1371/journal.pbio.1001961.s013

Snaidero, N., Möbius, W., Czopka, T., Hekking, L. H. P., Mathisen, C., Verkleij, D., Goebbels, S., Edgar, J., Merkler, D., Lyons, D. A., Nave, K.-A., \& Simons, M. (2014). Myelin Membrane Wrapping of CNS Axons by PI(3,4,5)P3-Dependent Polarized Growth at the Inner Tongue. Cell, 156(1-2), 277-290. http://doi.org/10.1016/j.cell.2013.11.044

Sofroniew, M. V. (2015). Astrocyte barriers to neurotoxicinflammation. Nature Reviews Neuroscience, 16(5), 249-263. http://doi.org/10.1038/nrn3898

Stys, P. K., Zamponi, G. W., van Minnen, J., \& Geurts, J. J. G. (2012). Will the real multiple sclerosis please stand up? Nature Reviews Neuroscience, 13(7), 507-514. http://doi.org/10.1038/nrn3275 
Sun, Z., Jin, P., Tian, T., Gu, Y., Chen, Y.-G., \& Meng, A. (2006). Activation and roles of ALK4/ALK7-mediated maternal TGF $\beta$ signals in zebrafish embryo. Biochemical and Biophysical Research Communications, 345(2), 694-703. http://doi.org/10.1016/j.bbrc.2006.04.148

Takada, N., Kucenas, S., \& Appel, B. (2010). Sox10 is necessary for oligodendrocyte survival following axon wrapping. Glia, 58(8), 996-1006. http://doi.org/10.1002/glia.20981

Taveggia, C., Feltri, M. L., \& Wrabetz, L. (2010). Signals to promote myelin formation and repair. Nature Reviews Neurology, 6(5), 276-287. http://doi.org/10.1038/nrneurol.2010.37

Taveggia, C., Zanazzi, G., Petrylak, A., Yano, H., Rosenbluth, J., Einheber, S., et al. (2005). Neuregulin-1 Type III Determines the Ensheathment Fate of Axons. Neuron, 47(5), 681-694. http://doi.org/10.1016/j.neuron.2005.08.017

Thomas, P. K. (1963). The connective tissue of peripheral nerve: an electron microscope study. Journal of Anatomy, 97, 35-44.

Thomas, P. K., \& Olsson, Y. (1984). Microscopic anatomy and function of the connective tissue components of the peripheral nerve. In: Dyck, P. J., Thomas, P. K., Lambert, E. H., Bunge, R., eds. Peripheral Neuropathy. $2^{\text {nd }}$ ed. Philadelphia, PA: Saunders; 2001:97-120. 
Tomassy, G. S., Dershowitz, L. B., \& Arlotta, P. (2016). Diversity Matters: A Revised Guide to Myelination. Trends in Cell Biology, 26(2), 135-147. http://doi.org/10.1016/j.tcb.2015.09.002

Topp, K. S., \& Boyd, B. S. (2006). Structure and biomechanics of peripheral nerves: nerve responses to physical stresses and implications for physical therapist practice. Physical Therapy, 86(1), 92-109.

Torii, T., Miyamoto, Y., Takada, S., Tsumura, H., Arai, M., Nakamura, K., Ohbuchi, K., Yamamoto, M., Tanoue, A., \& Yamauchi, J. (2014). In vivo knockdown of ErbB3 in mice inhibits Schwann cell precursor migration. Biochemical and Biophysical Research Communications, 452(3), 782-788.

Traka, M., Arasi, K., Avila, R. L., Podojil, J. R., Christakos, A., Miller, S. D., Soliven, B., \& Popko, B. (2010). A genetic mouse model of adult-onset, pervasive central nervous system demyelination with robust remyelination. Brain, 133(10), 30173029. http://doi.org/10.1093/brain/awq247

Traka, M., Podojil, J. R., McCarthy, D. P., Miller, S. D., \& Popko, B. (2015). Oligodendrocyte death results in immune-mediated CNS demyelination. Nature Neuroscience, 1-12. http://doi.org/10.1038/nn.4193 
Travnickova, J., Chau, V. T., Julien, E., Mateos-Langerak, J., Gonzalez, C., Lelièvre, E., Lutfalla, G., Tavian, M., \& Kissa, K. (2015). Primitive macrophages control HSPC mobilization and definitive haematopoiesis. Nature Communications, 6, 1-9. http://doi.org/10.1038/ncomms7227

Triarhou, L. C., \& Herndon, R. M. (1985). Effect of macrophage inactivation on the neuropathology of lysolecithin-induced demyelination. British Journal of Experimental Pathology, 66(3), 293-301.

Ullian, E. M., Sapperstein, S. K., Christopherson, K. S., \& Barres, B. A. (2001). Control of synapse number by glia. Science, 291(5504), 657-661. http://doi.org/10.1126/science.291.5504.657

Viganò, F., Möbius, W., Götz, M., \& Dimou, L. (2013). Transplantation reveals regionaldifferences in oligodendrocytedifferentiation in the adult brain. Nature Neuroscience, 16(10), 1370-1372. http://doi.org/10.1038/nn.3503

Wake, H., Lee, P. R., \& Fields, R. D. (2011). Control of local protein synthesis and initial events in myelination by action potentials. Science, 333(6049), 1647-1651. http://doi.org/10.1126/science.1206998

Wake, H., Moorhouse, A. J., \& Nabekura, J. (2012). Functions of microglia in the central nervous system - beyond the immune response. Neuron Glia Biology, 7(01), 47-53. http://doi.org/10.1038/nature05704 
Waksman, B. H., \& Adams, R. D. (1962). A histologic study of the early lesion in experimental allergic encephalomyelitis in the guinea pig and rabbit. The American Journal of Pathology, 41, 135-162.

Waller, A. (1850). Experiments on the section of the glossopharyngeal and hypoglossal nerves of the frog, and observations of the alterations produced thereby in the structure of their primitive fibres. Phil. Trans. R. Soc. Lond., 140, 423-429.

Wang, W.-D., Melville, D. B., Montero-Balaguer, M., Hatzopoulos, A. K., \& Knapik, E. W. (2011). Tfap2a and Foxd3 regulate early steps in the development of the neural crest progenitor population. Developmental Biology, 360(1), 173-185. http://doi.org/10.1016/j.ydbio.2011.09.019

Weinberg, H. J., Spencer, P. S., \& Raine, C. S. (1975). Aberrant PNS development in dystrophic mice. Brain Research, 88(3), 532-537.

Wiedenmann, J., Ivanchenko, S., Oswald, F., Schmitt, F., Röcker, C., Salih, A., Spindler, K.-D., \& Nienhaus, G. U. (2004). EosFP, a fluorescent marker protein with UVinducible green-to-red fluorescence conversion. Proceedings of the National Academy of Sciences of the United States of America, 101(45), 15905-15910. http://doi.org/10.1073/pnas.0403668101 
Wilkins, A., Majed, H., Layfield, R., Compston, A., \& Chandran, S. (2003). Oligodendrocytes promote neuronal survival and axonal length by distinct intracellular mechanisms: a novel role for oligodendrocyte-derived glial cell linederived neurotrophic factor. Journal of Neuroscience, 23(12), 4967-4974.

Winey, M., Meehl, J. B., O'Toole, E. T., \& Giddings, T. H. (2014). Conventional transmission electron microscopy. Molecular Biology of the Cell, 25(3), 319-323. http://doi.org/10.1091/mbc.E12-12-0863

Woldeyesus, M. T., Britsch, S., Riethmacher, D., Xu, L., Sonnenberg-Riethmacher, E., Abou-Rebyeh, F., Harvey, R., Caroni, P., \& Birchmeier, C. (1999). Peripheral nervous system defects in erbB2 mutants following genetic rescue of heart development. Genes \& Development, 13(19), 2538-2548.

Yamasaki, R., Lu, H., Butovsky, O., Ohno, N., Rietsch, A. M., Cialic, R., Wu, P. M., Doykan, C. E., Lin, J., Cotleur, A. C., Kidd, G., Zorlu, M. M., Sun, N., Hu, W., Liu, L., Lee, J.-C., Taylor, S. E., Uehlein, L., Dixon, D., Gu, J., Floruta, C. M., Zhu, M., Charo, I. F., Weiner, H. L., \& Ransohoff, R. M. (2014). Differential roles of microglia and monocytes in the inflamed central nervous system. Journal of Experimental Medicine, 211(8), 1533-1549. http://doi.org/10.1182/blood-2009-02200543 
Zawadzka, M., Rivers, L. E., Fancy, S. P. J., Zhao, C., Tripathi, R., Jamen, F., Young, K., Goncharevich, A., Pohl, H., Rizzi, M., Rowitch, D. H., Kessaris, N., Suter, U., Richardson, W. D., \& Franklin, R. J. M. (2010). CNS-Resident Glial Progenitor/Stem CellsProduce Schwann Cells as well as Oligodendrocytes during Repair of CNS Demyelination. Stem Cell, 6(6), 578-590. http://doi.org/10.1016/j.stem.2010.04.002

Zeisel, A., Muñoz-Manchado, A. B., Codeluppi, S., Lönnerberg, P., La Manno, G., Juréus, A., Marques, S., Munguba, H., He, L., Betsholtz, C., Rolny, C., CasteloBranco, G., Hjerling-Leffler, J., \& Linnarsson, S. (2015). Brain structure. Cell types in the mouse cortex and hippocampus revealed by single-cell RNA-seq. Science, 347(6226), 1138-1142. http://doi.org/10.1126/science.aaa1934

Zuchero, J. B., Fu, M.-M., Sloan, S. A., Ibrahim, A., Olson, A., Zaremba, A., Dugas, J. C., Wienbar, S., Caprariello, A. V., Kantor, C., Leonoudakis, D., LariosaWillingham, K., Kronenberg, G., Gertz, K., Soderling, S. H., Miller, R. H., \& Barres, B. A. (2015). CNS Myelin Wrapping Is Driven by Actin Disassembly. Developmental Cell, 34(2), 152-167. http://doi.org/10.1016/j.devcel.2015.06.011 